\title{
VALIDATION OF SCALE 4.0 - CSAS25 MODULE AND THE 27-GROUP ENDF/B-IV CROSS-SECTION LIBRARY FOR LOW-ENRICHED URANIUM SYSTEMS
}

\author{
W. C. Jordan
}

Computing and Telecommunications Division at Oak Ridge National Laboratory

P.O. Box 2008

Oak Ridge, TN 37831-6370

Date Published-February 1993

\begin{abstract}
NOTICE This document contains information of a preliminary nature. It is subject to revision or correction and sherefore does not represent a final report.
\end{abstract}

MARTIN MARIETTA ENERGY SYSTEMS, INC. managing the

Oak Ridge National Laboratory Oak Ridge Y-12 Plant Oak Ridge K-25 Plant Paducah Gaseous Diffusion Plant under Contract No. DE-AC05-84OR21400 for the

U.S. DEPARTMENT OF ENERGY 


\section{CONTENTS}

Page

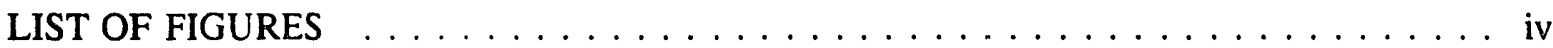

LIST OF TABLES $\ldots \ldots \ldots \ldots \ldots \ldots \ldots \ldots \ldots \ldots \ldots \ldots \ldots$

ABSTRACT $\ldots \ldots \ldots \ldots \ldots \ldots \ldots \ldots \ldots \ldots \ldots \ldots \ldots \ldots \ldots \ldots \ldots \ldots$

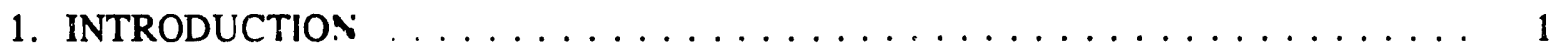

2. DESCRIPTION OF THE CODE PACKAGE $\ldots \ldots \ldots \ldots \ldots \ldots \ldots \ldots$

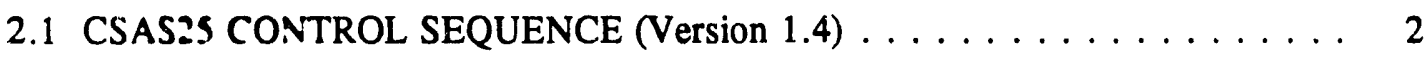

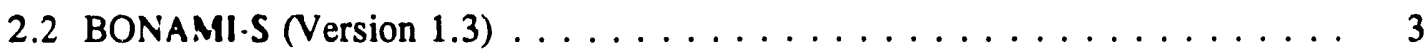

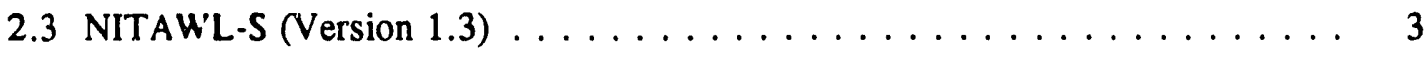

2.4 KENO V.a (Version 1.5) $\ldots \ldots \ldots \ldots \ldots \ldots \ldots \ldots \ldots \ldots \ldots$

2.5 THE 27-GROUP NEUTRON CROSS-SECTION LIBRARY (Rev. 2 created $1 / 27 / 89$, updated $10 / 12 / 89) \ldots \ldots \ldots \ldots \ldots$

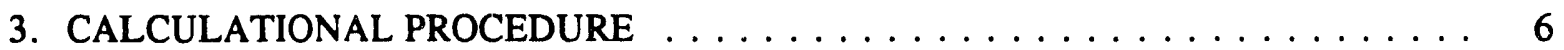

4. PROBLEM DESCRIPTION AND RESULTS $\ldots \ldots \ldots \ldots \ldots \ldots$

5. DISCUSSION AND CONCLUSIONS $\ldots \ldots \ldots \ldots \ldots \ldots \ldots \ldots$

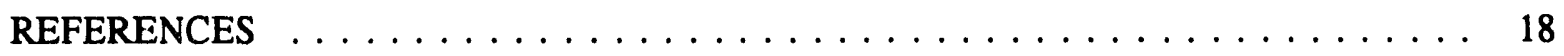

APPENDIX A. CSAS25 INPUT DATA USE FOR VALIDATION $\ldots \ldots \ldots \ldots \ldots$

APPENDIX B. CALCULATED RESULTS AND EXPERIMENTAL PARAMETERS $\ldots 115$

APPENDIX C. STATISTICAL ANALYSIS OF VALIDATION RESULTS $\ldots \ldots \ldots \ldots$ 


\section{LIST OF FIGURES}

\section{Figure}

Page

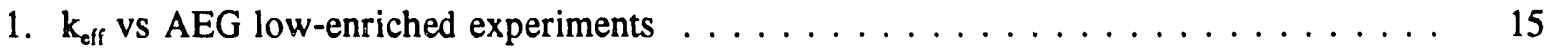

2. $\mathrm{k}_{\mathrm{eff}}$ vs AEG high- and low-enriched experiments $\ldots \ldots \ldots \ldots$ 


\section{LIST OF TABLES}

Table

$\underline{\text { Page }}$

1. KENO V.a calculated $k_{\text {eff }}$ for experimentally critical low-enriched uranium systems $\ldots .8$

2. KENO V.a calculated $k_{\text {eff }}$ for experimentally critical highly enriched uranium systems . 13

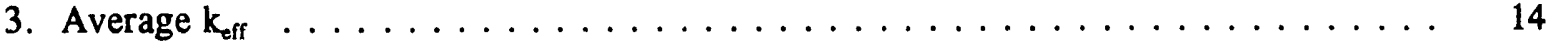




\begin{abstract}
A version of KENO V.a and the 27-group library in SCALE-4.0 were validated for use in evaluating the nuclear criticality safety of low-enriched uranium systems. A total of 59 critical systems were analyzed. A statistical analysis of the results was performed, and subcritical acceptanced criteria are established.
\end{abstract}




\section{INTRODUCTION}

The purpose of this report is to present the results of validation/reverification calculations using KENO V.a ${ }^{1}$ in SCALE 4.0. ${ }^{2}$ This validation is limited primarily to low-enriched uranium systems and is intended to demonstrate the ability of KENO V.a in the CSAS25 module ${ }^{3}$ to accurately calculate systems similar to those encountered at a low-enrichment Gaseous Diffusion Plant (GDP) and in the proposed Atomic Vapor Laser Isotope Separation (AVLIS) facility.

This validation was accomplished by performing calculations on critical systems for which the effective multiplication value, $\mathrm{k}_{\text {eff }}$, was known to be very close to 1.0. A statistical analysis was performed on the results of these calculations to determine a lower tolerance band that encompasses the range of calculated $k_{\text {eff }}$ values which belong to a group of critical systems. ${ }^{4}$ The statistical treatment provides a high degree of confidence that a calculation that yields a value of $k_{\text {eff }}$ below the lower tolerance band is not of a critical system. This lower tolerance band is then used as an upper safety limit for subcriticality.

A total of 59 critical systems were analyzed in this validation. The models of 51 critical systems were selected from a previous validation of KENO V.a reported in ORNL/CSD/TM-238. ${ }^{5}$ Eleven of these 51 models were of highly enriched systems included to demonstrate the ability of the codes to accurately calculate systems over the full enrichment range and to validate the ${ }^{235} \mathrm{U}$ cross sections. Forty of the models were of low-enriched uranium systems which allow the demonstration of this ability to accurately calculate a broad range of low-enriched systems. Models of 9 lowenriched reactor lattices previously analyzed by H. R. Dyer were included to allow testing the adequacy of the code and cross sections for heterogeneous systems. ${ }^{6}$

A description of the CSAS25 sequence and the major changes that have been made in the various programs are given in Sect. 2. The calculational procedure is given in Sect. 3, and problem descriptions and results are given in Sect. 4.

Section 5 presents a discussion of the results of the calculation. A statistical analysis of the results is presented, and subcriticality acceptance criteria are established. 


\section{DESCRIPTION OF THE CODE PACKAGE}

This report documents the validation of SCALE 4.0 on the ORNL IBM-3090 computer as it existed in November 1990. This version is under configuration management plan SCALE-CMP001, Rev. 1.7 The programs in SCALE 4.0 are not strictly "frozen"; however, they are under stringent configuration change control. This control allows elimination of code errors in a timely manner while introducing minimal concerns for the ability of the programs to perform their prescribed functions. The following subsections give a brief description of the program modules that were validated. The version number of each program is included in the following discussions. The version numbers of the program modules should be checked when calculations are performed to verify that the validated versions are being used. If a code version changes, the nature of the modifications should be reviewed and check cases should be run to see if complete revalidation is warranted.

\subsection{CSAS25 SEQUENCE (VERSION 1.4)}

This report documents only the CSAS25 control sequence of the CSAS4 control module in SCALE 4.0. The CSAS25 control sequence reads user-specified data, which include the required cross-section library, specifications for mixtures, information for resonance region cross-section processing of nuclides (size, geometry, and temperature), and a detailed geometry model for KENO V.a. Physical and neutronics information not supplied explicitly but required by the functional modules (such as theoretical density, molecular weights, average resonance region background cross sections) is supplied by the Standard Composition Library ${ }^{8}$ and calculated by the Material Information Processor Library (MIPLIB). ${ }^{9}$ These data are used by CSAS25 to set up the input for BONAMI-S, ${ }^{10}$ NITAWL-S, ${ }^{11}$ and KENO V.a. Several significant modifications and enhancements have been developed for the CSAS25 control sequence in SCALE 4.0, compared with earlier versions. These modifications and their impact are described below.

Modifications were made to MIPLIB, called by CSAS25, which impact the input data for NITAWL. An approximation was incorporated into MIPLIB which treats all admixed moderators in a mixture. This modification eliminated a $1.5 \%$ positive bias for a series of low-enriched green block experiments, but had little effect on other unbiased low- and high-enriched systems.

Modifications were made to MIPLIB when calculating the Dancoff factor for lattices. The modifications apply a conservative adjustment to the Dancoff factor, eliminating a $50 \%$ negative bias for systems in which the mean-free path of the neutron is large with respect to the lattice spacing. This correction has little effect on typical reactor lattices.

Modifications were made to MIPLIB in order to implement an annular treatment in NITAWL. The addition of the annular geometry treatment into NITAWL and the CSAS25 module represents a significant code enhancement. This treatment is implemented automatically for LATTICECELL and MULTIREGION calculations. The annular option may be invoked specifically in LATTICECELL calculations by specifying one of the annular geometries; however, the annular treatment is used in all LATTICECELL geometry specifications. Similarly, the annular geometry treatment is used for all MULTIREGION calculations. The external moderator indices that flag the type of treatment to be used in MULTIREGION calculations are optional input data in SCALE 4.0. In the annular geometry implementation, an inward and outward transmission probability are calculated. These probabilities are used to obtain the Dancoff factor for the region being treated. Each geometry region is treated independently. A "reasonable" resonance correction is performed for every region. In previous versions of SCALE, certain assumptions had been made about where 
the resonance absorbers were located and how they would be treated. These assumptions had the potential of causing a significant error in the resonance correction applied to resonance absorbers that were modeled in a region which had not been anticipated (for example, in the clad region or moderator region of LATTICECELL problems).

Modifications were made to MIPLIB to improve the estimation of the specific gravity of uranyl nitrate solutions. An option was added to allow the specific gravity or density to be entered for any of the solutions or materials in the Standard Composition Library.

\subsection{BONAMI-S (VERSION 1.3)}

BONAMI-S performs resonance shielding through the application of the Bondarenko shielding factor method. BONAMI-S reads the master-format cross-section library and applies the Bondarenko correction to all nuclides that have Bondarenko data. Input to BONAMI-S, set up by the CSAS25 control sequence, includes information relating to the physical characteristics (composition of material, size, geometry, temperature) of the system being calculated. BONAMI-S produces a Bondarenko-corrected master-format library that is read by NITAWL-S. For the 27group master cross-section library used in this validation, the primary purpose of the BONAMI functional module is to select the required material cross sections and to create a smaller master cross-section library to be processed by NITAWL-S. No data processing is performed in BONAMI for the 27-group cross-section library.

\subsection{NITAWL-S (VERSION 1.3)}

NITAWL-S applies the Nordheim Integral Treatment to perform neutron cross-section processing in the resolved resonance range for nuclides that have single-level Breit-Wigner (SLBW) resonance parameter data. This technique involves the numerical integration of SLBW resonance parameters using a calculated flux distribution that is based on the collision density across each resonance and subsequent weighting of the cross-section to the desired broad-group structure. Input data to NITAWL-S, automatically set up by the CSAS25 control sequence, include information relating to the physical and neutronic characteristics of the system being calculated. NITAWL-S uses these data to complete the processing of the problem-dependent master library from BONAMI-S. NITAWL-S assembles the group-to-group transfer arrays from the elastic and inelastic scattering components and performs other tasks to produce a problem-dependent, working format cross-section library that can be used by KENO V.a. The analyst specifies the input resonance data based on the available options. The options include (1) a homogeneous medium treatment that treats the resonance region of the fissile mixture as if it were an infinite homogeneous media and (2) a finite lump treatment that treats the resonance region as if the fissile mixture were a discrete lump with a $1 / E$ return flux at the boundary. This latter option is for problems where the system is substantially heterogeneous or where reflector effects are important. These options are automatically invoked in the CSAS25 control sequence according to the keywords INFHOMMEDIUM, LATTICECELL, or MULTIREGION. The INFHOMMEDIUM option treats the fissile mixture as an infinite homogeneous medium. The LATTICECELL option uses a multiple repeated cell for the resonance self-shielding correction. With the MULTIREGION option, a single-cell resonance selfshielding calculation is made. 
Several modifications have been made to NITAWL-S. These include the addition of the ability to more accurately treat annular geometry and the ability to read and use the new AMPX ${ }^{12}$ master-format libraries. Previous AMPX master libraries had the resonance region cross sections referenced to background cross sections such that the contribution from the resonances calculated by NITAWL was added to the background to obtain the shielded values. In the SCALE 4.0 AMPX master-format libraries, the cross sections are referenced to infinite dilution cross sections so the contribution from the resonances calculated by NITAWL is subtracted from the infinite dilution values to obtain the shielded values. This change was made to decouple the resonance region crosssection reconstruction from the codes used to generate the cross-section data (in this case, XLACS ${ }^{13}$ ). As a result of these changes, a larger correction is required for pure uranium metal systems than for dilute uranium mixtures. The NITAWL annular treatment is discussed in Sect. 2.1.

\subsection{KENO V.a (VERSION 1.5)}

KENO V.a is a multigroup Monte Carlo code used to determine $k_{\text {eff }}$. KENO V.a is a substantial revision of KENO IV and includes an enhanced geometry package that allows modeling of a wide variety of complex three-dimensional (3-D) geometries. The geometry package allows nested arrays and "holes" to be placed in the geometry model. The code permits the use of reflector options, including mirror reflection, differential albedo reflection, and an automatic reflector; the latter has the capability of using reflector region-weighting functions that are based on onedimensional (1-D) adjoint calculations. The reflector options simplify geometry data input and/or reduce the running time of a specific problem. Cross-section input and atom-density input are handled by the CSAS25 module. Most of the major KENO parameters have defaults that work for a wide variety of problems, but can be overridden if the problem or analyst dictates.

There have been many minor changes to the KENO V.a code, most of which are invisible to the user. When a code problem arises in KENO, the run typically terminates. Several changes to the data checking performed by KENO and to program logic involved in the treatment of computer round-off error during tracking have been made.

Changes have also been made in the manner in which KENO handles inadequate Legendre transfer cross-section expansions. Inadequate Legendre expansions may be caused by a number of problems including poor cross-section data, inadequate or incompatible processing of the base data, and/or roundoff of the data. When inadequate Legendre expansions are encountered by KENO, they are corrected by the smallest amount required to make them usable.

\subsection{THE 27-GROUP NEUTRON CROSS-SECTION LIBRARY}

(Rev. 2 created January 27, 1989, updated October 12, 1989)

The 27-group ENDF/B-IV master cross-section library ${ }^{14}$ in SCALE is activated in the CSAS25 control sequence by specifying 27GROUPNDF4 (27GR) as the cross-section library name. The 27-group library is the broad-group companion library to the 218-group Criticality Safety Reference Library. The Criticality Safety Reference Library master library, which is based on ENDF/B-IV data, was generated as a pseudo-problem-independent fine-group structure library for use in general criticality safety analysis and shipping cask calculations. The 27 -group library was collapsed from the 218-group library using a characteristic fission-(1/E)-Maxwellian spectral flux shape. Explicit ENDF/B-IV resonance parameters are carried for resonance nuclides in both the 27-group and 218-group master libraries. These resonance parameters are used by NITAWL-S in 
the CSAS25 control sequence for calculating problem-dependent, self-shielded resonance region cross sections.

The original 27-group library was processed into the new AMPX master-library format using CORECTOL. ${ }^{15}$ In addition, all SCALE-4.0 nuclides were processed with PERFUME. ${ }^{16}$ The cross sections distributed with SCALE 4.0 are not compatible with previous versions of SCALE. 


\section{CAlCUlational PROCEDURe}

In the KENO V.a validation process, many of the available options in CSAS25 and KENO V.a were exercised. These options include the automatic atomic density generation features, the various treatments in NITAWL, and the geometry and reflector options in KENO V.a. This procedure was followed in order to validate the mechanics and capabilities of the code for a broad spectrum of problems. The majority of the calculations repeat calculations that were modeled by different people at different times. Usually there are multiple ways that a problem may be specified, all of which are equally correct. The original input data were left substantially unchanged in order to allow testing for discrepancies or bias that might have been caused by the differences in analysts' problem specifications. A complete listing of the input data has been included in Appendix A.

The resonance treatment used was the one that appeared to most accurately fit the problem specifications. All problems were run for 103 generations of 600 neutrons per generation. Some problems were run using the adjoint reflector weighting (bias ID), whereas others were run using full neutron tracking in the reflector in order to test for bias which might be caused by one or the other treatment.

Vyith KENO V.a, there is no single $k_{\text {eff }}$ that is "the answer" for the problem. The code starts with an initial neutron distribution and calculates the effective multiplication for a generation of neutrons. The next generation uses a starting distribution based on the fission points of the previous generation and caiculates the effective multiplication for the current generation. Because 103 generations were run, there are 103 different answers In the ideal situation, once the source has converged and the original (and usually arbitrary) starting distribution has decayed, the difference in the batch-to-batch $\mathrm{k}_{\text {eff }}$ may be attributed to the statistical nature of the Monte Carlo calculation, and the "best" answer should be the average of the remaining calculations. The common practice in KENO V.a is to tabulate the average $k_{\text {eff }}$ as a function of the number of generations skipped. Considerable judgment is then required in determining the answer. Many calculations do not approach the ideal situation. For example, undersampling may exist where the number of neutrons per generation is not sufficient to sustain a proper source distribution, or the number of generations is insufficient to converge the source distribution. In order to remove the element of judgment from the interpretation of the results and to mechanize the process of validating and comparing results with previous validation, the average $k_{\text {eff }}$ by generation skipped with the lowest calculated standard deviation is reported here. This number appears in the plot of average $k_{\text {eff }}$ by generation skipped in the KENO V.a output.

In each calculation, the optional parameter N'P was set to "yes" in KENO V.a, so the program would calculate and print the average energy group of the neutron-causing fission. This parameter has been frequently used in past validations because of trends that have been observed and appear to be correlated. 


\section{PR.OBLEM DESCRIPTION AND RESULTS}

A brief problem description and results are presented in Tables 1 and 2. The majority of the models used in this validation were taken from ORNL/CSD/TM-238. The descriptions in Tables 1 and 2 are organized in a manner to refer back to the tables in ref. 5 .

The experiments analyzed were chosen to validate KENO V.a in SCALE 4.0 for lowenriched uranium systems similar to those encountered at the GDP and in the proposed AVLIS facility.

The experiments described in Table I, Section A, are 4.89\% ${ }^{235} \mathrm{U}$ enriched uranium systems from Table 1 in ref. 5. The descriptions of the models for the $4.89 \%$ uranium metal rod experiments were obtained from the original data notebooks. Geometries modeled include single-unit spheres and slabs and arrays of cylinders. The fissile compounds considered were $\mathrm{UO}_{2} \mathrm{~F}_{2}, \mathrm{U}$ metal, and $\mathrm{U}_{3} \mathrm{O}_{8}$. Moderating materials were water, sterotex, and $\mathrm{UO}_{2} \mathrm{~F}_{2}$ solution.

The experimental models used to generate Table 1, Sect. B, are from low-enriched uranium experiments obtained from Table 2 in ref. 5. These experiments are formally undocumented experiments for $3.85 \%$ enriched uranium metal rods in water. The heterogeneous systems consist of large-diameter rods that are water moderated. In some cases, the rods have an internal hole which is also water moderated. The critical experiments were modeled in detail from descriptions in the experiment logbook. Extensive detail was included in these calculations in order to thoroughly test the KENO V.a geometry package.

Table 1, Sect. C, includes models from Table 3 in ref. 5 which were used for the validation of Y12CSG for the Oak Ridge Gaseous Diffusion Plant (ORGDP). The validation considered singleunit criticals, both reflected and unreflected, at several enrichments ranging from 1.4 to $4.98 \%$. The first 14 experiments are $\mathrm{UF}_{4}$ /paraffin-moderated systems and $\mathrm{UO}_{2} \mathrm{~F}_{2}$ solution systems at various moderation levels. The last 10 experiments are $4.46 \%$ enriched damp-oxide experiments performed at Rocky Flats. These later experiments test the code's ability to handle both intermixed and interstitial moderation. Several of the damp oxide experiments, which were driven by highly enriched uranium systems, were added to the series.

Table 1, Sect. D, models were taken from ref. 6. These experiments are arrays of $2.459 \mathrm{wt}$ $\%{ }^{235} \mathrm{U} \mathrm{UO}_{2}$ fuel pins in array of arrays with borated water as the moderator. These experiments were chosen because (1) they test the ability of the codes and cross sections to calculate systems with a harder neutron spectrum and (2) they had exhibited a bias in the past.

Several highly enriched uranium experiments were included in the validation. Thesa models, taken from Tables 4 and 5 in ref. 5, were included to check the code's ability to properly calculate resonance cross sections for low-moderated and unmoderated systems. Several deficiencies in the early implementation of NITAWL-II were discovered when calculating highly enriched systems. The deficiencies were related to correcting infinite dilute cross sections $\left(\sigma_{\mathrm{p}}=\infty\right)$ to near pure material values $\left(\sigma_{\mathrm{p}}=\sigma_{\mathrm{r}}\right)$. 
Table 1. KENO V.a calculated $k_{\text {eff }}$ for experimentally critical low-enriched uranium systems

\begin{tabular}{|c|c|c|c|}
\hline Case & Experimental description & Reference & $k_{\text {eff }} \pm \sigma$ \\
\hline \multicolumn{4}{|c|}{ A) Ref. 5, Table 1 cases } \\
\hline CAA01 & $\begin{array}{l}\text { Experiment } 1 \mathrm{~A} .401-4.89 \% \mathrm{U} \text { rods, } 0.762-\mathrm{cm} \text { diam, } \\
30-\mathrm{cm} \text { long, } 1.3-\mathrm{cm} \text { pitch, } \mathrm{H}_{2} \mathrm{O} \text { moderated and } \\
\text { reflected }\end{array}$ & 17 & $0.9894 \pm 0.0040$ \\
\hline CAA04 & $\begin{array}{l}\text { Experiment } 6 \mathrm{~A} .203-4.89 \% \mathrm{U} \text { rods, } 0.762-\mathrm{cm} \text { diam, } \\
30-\mathrm{cm} \text { long, } 2.05-\mathrm{cm} \text { pitch, } \mathrm{H}_{2} \mathrm{O} \text { moderated and } \\
\text { reflected }\end{array}$ & 17 & $0.9893 \pm 0.0032$ \\
\hline CAA07 & $\begin{array}{l}\text { Experiment } 23 \mathrm{~B} .400-4.89 \% \mathrm{U} \text { rods, } 0.762-\mathrm{cm} \text { diam, } \\
30 \text {-cm long, } 1.3-\mathrm{cm} \text { pitch, } \mathrm{H}_{2} \mathrm{O} \text { moderated, } \mathrm{H}_{2} \mathrm{O} \text { top } \\
\text { reflector, Plexiglas bottom reflector, } \mathrm{Pb} \text { reflected } 4 \\
\text { faces }\end{array}$ & 17 & $0.9806 \pm 0.0032$ \\
\hline $\mathrm{CAA} 12$ & $\begin{array}{l}\text { Experiment } 32 \mathrm{~S} .11 \times 12 \text { array of } 4.89 \% \mathrm{U} \text { rods in } \\
\sim 300 \mathrm{~g} \mathrm{U} / \mathrm{L} \mathrm{UO}_{2} \mathrm{~F}_{2} \text { solution, } 0.762-\mathrm{cm} \text { diam, } 2.05-\mathrm{cm} \\
\text { pitch, in } 95.88-\mathrm{cm} \text {-diam tank }\end{array}$ & 17 & $0.9793 \pm 0.0025$ \\
\hline CAA14 & $\begin{array}{l}\text { Experiment } 36 \mathrm{~S} .9 \times 10 \text { array of } 4.89 \% \mathrm{U} \text { rods in } \\
\sim 300 \mathrm{~g} \mathrm{U} / \mathrm{L} \mathrm{UO}_{2} \mathrm{~F}_{2} \text { solution, } 0.762-\mathrm{cm} \text {-diam, } 2.453 \text { - } \\
\mathrm{cm} \text { pitch, in } 95.88 \text {-cm-diam tank }\end{array}$ & 17 & $0.9893 \pm 0.0032$ \\
\hline CAA19 & $\begin{array}{l}4.89 \% \mathrm{U}_{3} \mathrm{O}_{8} \text { - Sterotex blocks, } 55.5 \mathrm{~g}^{235} \mathrm{U} / \mathrm{L} \text {, } \\
\text { unreflected }\end{array}$ & 18 & $0.9913 \pm 0.0037$ \\
\hline CAA21 & $\begin{array}{l}4.89 \% \mathrm{U}_{3} \mathrm{O}_{8}-\text { Sterotex blocks, } 22.1 \mathrm{~g}{ }^{235} \mathrm{U} / \mathrm{L} \\
(\mathrm{H} / \mathrm{X}=757.0), \text { unreflected }\end{array}$ & 18 & $0.9997 \pm 0.0033$ \\
\hline CAA25 & $\begin{array}{l}4.89 \% \mathrm{U}_{3} \mathrm{O}_{8}-\text { Sterotex blocks, } 81.1 \mathrm{~g}{ }^{235} \mathrm{U} / \mathrm{L}(\mathrm{H} / \mathrm{X}= \\
396.7) \text {, paraffin top reflector, } \mathrm{H}_{2} \mathrm{O} \text {-reflected bottom } \\
\text { and sides }\end{array}$ & 18 & $0.9999 \pm 0.0032$ \\
\hline CAA27 & $\begin{array}{l}4.89 \% \mathrm{U}_{3} \mathrm{O}_{8}-\mathrm{Sterotex} \text { blocks, } 81.1 \mathrm{~g}^{235} \mathrm{U} / \mathrm{L}(\mathrm{H} / \mathrm{X}= \\
146.8) \text {, paraffin top reflector, } \mathrm{H}_{2} \mathrm{O} \text {-reflected bottom } \\
\text { and sides }\end{array}$ & 18 & $0.9873 \pm 0.0034$ \\
\hline CAA29 & $\begin{array}{l}4.89 \% \mathrm{U}_{3} \mathrm{O}_{8} \text { - Sterotex blocks, } 33.3 \mathrm{~g}{ }^{235} \mathrm{U} / \mathrm{L}(\mathrm{H} / \mathrm{X}= \\
504.1) \text {, paraffin top reflector, } \mathrm{H}_{2} \mathrm{O} \text {-reflected bottom } \\
\text { and sides }\end{array}$ & 18 & $0.9860 \pm 0.0031$ \\
\hline CAA33 & $\begin{array}{l}4.89 \% \mathrm{UO}_{2} \mathrm{~F}_{2} \text { solution } 24.04 \mathrm{~g}^{235} \mathrm{U} / \mathrm{L}(\mathrm{H} / \mathrm{X}=1002) \\
\text { in a } 27.3 \text {-in.-diam aluminum sphere, unreflected }\end{array}$ & 18 & $0.9916 \pm 0.0024$ \\
\hline CAA38 & $4.89 \% \mathrm{UO}_{2} \mathrm{~F}_{2}$ solution $22.11 \mathrm{~g}^{235} \mathrm{U} / \mathrm{L}(\mathrm{H} / \mathrm{X}=991)$ in & 18 & $1.0000 \pm 0.0025$ \\
\hline
\end{tabular}
a 27.3-in.-diam aluminum sphere, $\mathrm{H}_{2} \mathrm{O}$ reflected 
Table 1 (continued)

\begin{tabular}{llll}
\hline Case & Experimental description & Reference & $k_{\text {eff }} \pm \sigma$ \\
\hline
\end{tabular}

B) Ref. 5, Table 2 cases

CAB03 (EBJ.3X) lattice 8. 24-3.85\% U rods, 7.2-in.-diam $\times$

30-in.-long square lattice, 8.70 in. center to center, $75.0-\mathrm{cm}$ water height

CAB08 (EBJ.5X) lattice 9. 7-3.85\% U rods, 7.2 in. diam $\times$ $30 \mathrm{in}$. long in triangular lattice, 8.20 in. center to center, $53.1 \mathrm{~cm}$ water height

CAB11 (EBJ.9) lattice 6. 16-3.85\% U rods, 7.2 in. OD $\times$ 2.6 in. ID $\times 30$ in. long in square lattice, 8.70 in. center to center, $49.2 \mathrm{~cm}$ water height

CAB15 (EBJ.13) lattice 9. 15-3.85\% U rods, 2.5 in. diam $\times$ 30 in. long in square lattice, 3.25 in. center to center, $64.8 \mathrm{~cm}$ water height

C) Ref. 5, Table 3 cases

CASO4 An unreflected rectangular parallelepiped of homogeneous $\mathrm{U}(1.4) \mathrm{F}_{4}$ and paraffin with an $\mathrm{H} /{ }^{235} \mathrm{U}$ atomic ratio of $421.8 ; 93.1 \mathrm{~cm} \times 93.0 \mathrm{~cm} \times 123.8$ cm

CAS06 An unreflected rectangular parallelepiped of homogeneous $\mathrm{U}(1.4) \mathrm{F}_{4}$ and paraffin with an $\mathrm{H} /{ }^{235} \mathrm{U}$ atomic ratio of $421.8 ; 130.7 \mathrm{~cm} \times 130.6 \mathrm{~cm} \times 74.2$ cm

CAS11 A reflected rectangular parallelepiped of homogeneous $\mathrm{U}(2) \mathrm{F}_{4}$ and paraffin with an $\mathrm{H} /{ }^{235} \mathrm{U}$ atomic ratio of $195.2 ; 56.22 \mathrm{~cm} \times 56.22 \mathrm{~cm} \times 112.88$ $\mathrm{cm}$, reflected with $15.2 \mathrm{~cm}$ of paraffin on top and sides and $15.2 \mathrm{~cm}$ of Plexiglas on the bottom

CAS16 A reflected rectangular parallelepiped of homogeneous $\mathrm{U}(2) \mathrm{F}_{4}$ and paraffin with an $\mathrm{H} /{ }^{235} \mathrm{U}$ atomic ratio of $495.9 ; 46.00 \mathrm{~cm} \times 46.00 \mathrm{~cm} \times 96.57$ $\mathrm{cm}$, reflected with $15.2 \mathrm{~cm}$ of paraffin on top and sides of $15.2 \mathrm{~cm}$ of Plexiglas on the bottom

CAS17 A reflected rectangular parallelepiped of homogeneous $\mathrm{U}(2) \mathrm{F}_{4}$ and paraffin with an $\mathrm{H} /{ }^{235} \mathrm{U}$ atomic ratio of $613.6 ; 56.32 \mathrm{~cm} \times 61.29 \mathrm{~cm} \times 54.08$ $\mathrm{cm}$, reflected with $15.2 \mathrm{~cm}$ of polyethylene on top and sides and $15.2 \mathrm{~cm}$ of Plexiglas on the bottom 
Table 1 (continued)

\begin{tabular}{ll}
\hline Case & \multicolumn{1}{c}{ Experimental description } \\
\hline CAS21 & $\begin{array}{l}\text { A reflected rectangular parallelepiped of } \\
\text { homogeneous } \mathrm{U}(3) \mathrm{F}_{4} \text { and paraffin with an } \mathrm{H} /{ }^{235} \mathrm{U} \\
\text { atomic ratio of } 133.4 ; 51.14 \mathrm{~cm} \times 51.14 \mathrm{~cm} \times 51.27 \\
\\
\text { cm, reflected with } 15.2 \mathrm{~cm} \text { of paraffin on top and } \\
\text { sides and } 15.2 \mathrm{~cm} \text { of Plexiglas on the bottom }\end{array}$ \\
CAS23 & $\begin{array}{l}\text { A reflected rectangular parallelepiped of } \\
\text { homogeneous } \mathrm{U}(3) \mathrm{F}_{4} \text { and paraffin with an } \mathrm{H} /{ }^{235} \mathrm{U}\end{array}$ \\
& $\begin{array}{l}\text { atomic ratio of } 133.4 ; 46.02 \mathrm{~cm} \times 46.02 \mathrm{~cm} \times 67.57 \\
\mathrm{~cm}, \text { reflected with } 15.2 \mathrm{~cm} \text { of paraffin on top and } \\
\text { sides and } 15.2 \mathrm{~cm} \text { of Plexiglas on the bottom }\end{array}$
\end{tabular}

CAS26 An unreflected rectangular parallelepiped of homogeneous $\mathrm{U}(3) \mathrm{F}_{4}$ and paraffin with an $\mathrm{H} /{ }^{235} \mathrm{U}$ atomic ratio of $133.4 ; 56.47 \mathrm{~cm} \times 56.47 \mathrm{~cm} \times 86.64$ cm

CAS29 A reflected rectangular parallelepiped of homogeneous $\mathrm{U}(3) \mathrm{F}_{4}$ and paraffin with an $\mathrm{H} /{ }^{235} \mathrm{U}$ atomic ratio of $276.9 ; 40.81 \mathrm{~cm} \times 40.80 \mathrm{~cm} \times 39.49$ $\mathrm{cm}$, reflected with $15.2 \mathrm{~cm}$ of polyethylene on top and sides and $15.2 \mathrm{~cm}$ of Plexiglas on the bottom

CAS31 An unreflected rectangular parallelepiped of homogeneous $\mathrm{U}(3) \mathrm{F}_{4}$ and paraffin with an $\mathrm{H} /{ }^{235} \mathrm{U}$ atomic ratio of $276.9 ; 48.59 \mathrm{~cm} \times 51.14 \mathrm{~cm} \times 48.53$ cm

CAS33 A composite cadmium/steel/water side reflected stainless steel cylinder of $0.079-\mathrm{cm}$ wall thickness and $19.545-\mathrm{cm}$ IR filled to a height of $54.45 \mathrm{~cm}$ with $\mathrm{U}(4.98) \mathrm{O}_{2} \mathrm{~F}_{2}$ solution at an $\mathrm{H} /{ }^{235} \mathrm{U}$ atomic ratio of 488

$21 \quad 1.0124 \pm 0.0032$

$21 \quad 1.0072 \pm 0.0028$

$21 \quad 1.0070 \pm 0.0032$

$21 \quad 1.0043 \pm 0.0029$

$22 \quad 0.9934 \pm 0.0028$

$22 \quad 0.9915 \pm 0.0031$

A composite 1-in. steel/water side reflected steel
cylinder of $0.079-\mathrm{cm}$ wall thickness and $16.51-\mathrm{cm}$ IR filled to a height of $143 \mathrm{~cm}$ with $\mathrm{U}(4.98) \mathrm{O}_{2} \mathrm{~F}_{2}$ solution at an $\mathrm{H} /{ }^{235} \mathrm{U}$ atomic ratio of 488

CAS36 An unreflected stainless steel cylinder of $0.07874 \mathrm{~cm}$ wall thickness and a 19.55-cm IR filled to a height of $101.7 \mathrm{~cm}$ with $\mathrm{U}(4.98) \mathrm{O}_{2} \mathrm{~F}_{2}$ solution at an $\mathrm{H} /{ }^{235} \mathrm{U}$ atomic ratio of 496

CAR01 Experiment 1. $4.46 \%$ enriched $\mathrm{U}_{3} \mathrm{O}_{8}, \mathrm{H} / \mathrm{U}=0.77$, 42 fuel cans with $2.44 \mathrm{~cm}$ interstitial moderation, plastic reflected 
Table 1 (continued)

\begin{tabular}{|c|c|c|c|}
\hline Case & Experimental description & Reference & $\mathrm{k}_{\mathrm{eft}} \pm \sigma$ \\
\hline CAR04 & $\begin{array}{l}\text { Experiment } 13.4 .46 \% \text {-enriched } \mathrm{U}_{3} \mathrm{O}_{8}, \mathrm{H} / \mathrm{U}=0.77 \text {, } \\
40 \text { fuel cans with } 2.44-\mathrm{cm} \text { interstitial moderation, } \\
\text { concrete reflected }\end{array}$ & 24 & $1.0116 \pm 0.0032$ \\
\hline CAR07 & $\begin{array}{l}4.46 \% \text {-enriched } \mathrm{U}_{3} \mathrm{O}_{8}, \mathrm{H} / \mathrm{U}=0.77 \text {, driven by high } \\
\text { concentration }(14.844 \mathrm{~kg} 351.18 \mathrm{~g} / \mathrm{L}) \text { fuel cans, } \\
\text { plastic reflected }\end{array}$ & 25 & $1.0158 \pm 0.0037$ \\
\hline CAR08 & $\begin{array}{l}4.46 \% \text {-enriched } \mathrm{U}_{3} \mathrm{O}_{8}, \mathrm{H} / \mathrm{U}=0.77 \text {, driven by low } \\
\text { concentration }(12.871 \mathrm{~kg} 86.42 \mathrm{~g} / \mathrm{L}) 93.17 \% \text { enriched } \\
\mathrm{UO}_{2}\left(\mathrm{NO}_{3}\right)_{2}, 119+2 \mathrm{~S} \text { fuel cans, plastic reflected }\end{array}$ & 25 & $1.0017 \pm 0.0035$ \\
\hline CAR11 & $\begin{array}{l}\text { Experiment A. } 4.46 \% \text {-enriched } \mathrm{U}_{3} \mathrm{O}_{8} \mathrm{H} / \mathrm{U}=1.25 \text {, } \\
38 \text { fuel cans with } 2.44-\mathrm{cm} \text { interstitial moderation, } \\
\text { plastic reflected }\end{array}$ & 26 & $1.0070 \pm 0.0033$ \\
\hline CAR13 & $\begin{array}{l}\text { Experiment } C .4 .46 \% \text {-enriched } \mathrm{U}_{3} \mathrm{O}_{8} \mathrm{H} / \mathrm{U}=1.25 \text {, } \\
80 \text { fuel cans with } 0.929-\mathrm{cm} \text { interstitial moderation, } \\
\text { plastic reflected }\end{array}$ & 26 & $1.0081 \pm 0.0030$ \\
\hline CAR15 & $\begin{array}{l}4.46 \% \text {-enriched } \mathrm{U}_{3} \mathrm{O}_{8} \mathrm{H} / \mathrm{U}=1.25 \text {, driven by high } \\
\text { concentration }(12.400 \mathrm{~kg} 351.65 \mathrm{gU} / \mathrm{L}), 93.17 \% \\
\text { enriched } \mathrm{UO}_{2}\left(\mathrm{NO}_{3}\right)_{2}, 119+2 \mathrm{~S} \text { fuel cans, plastic } \\
\text { reflected }\end{array}$ & 26 & $0.9995 \pm 0.0030$ \\
\hline CAR16 & $\begin{array}{l}4.46 \% \text {-enriched } \mathrm{U}_{3} \mathrm{O}_{8} \mathrm{H} / \mathrm{U}=1.25 \text {, driven by low } \\
\text { concentration }(10.836 \mathrm{~kg} \text { p } 86.60 \mathrm{gU} / \mathrm{L}), 93.17 \% \\
\text { enriched } \mathrm{UO}_{2}\left(\mathrm{NO}_{3}\right)_{2}, 119+2 \mathrm{~S} \text { fuel cans, plastic } \\
\text { reflected }\end{array}$ & 26 & $1.0090 \pm 0.0034$ \\
\hline CAR18 & $\begin{array}{l}\text { Experiment } \mathrm{G} \text {. } 4.46 \% \text {-enriched } \mathrm{U}_{3} \mathrm{O}_{8} \mathrm{H} / \mathrm{U}=2.03 \text {, } \\
30 \text { fuel cans with } 2.44-\mathrm{cm} \text { interstitial moderation, } \\
\text { plastic reflected }\end{array}$ & 27 & $1.0087 \pm 0.0031$ \\
\hline CAR20 & $\begin{array}{l}\text { Experiment } \mathrm{E} .4 .46 \% \text {-enriched } \mathrm{U}_{3} \mathrm{O}_{8} \mathrm{H} / \mathrm{U}=2.03 \text {, } \\
\text { driven by } 93.12 \% \text { enriched hollow uranium metal } \\
\text { sphere }(12.786 \mathrm{~kg}), 120+4 \mathrm{~S} \text { fuel cans, plastic } \\
\text { reflected }\end{array}$ & 27 & $1.0036 \pm 0.0026$ \\
\hline
\end{tabular}


12

Table 1 (continued)

\begin{tabular}{|c|c|c|c|}
\hline Case & Experimental description & Reference & $k_{\text {eff }} \pm \sigma$ \\
\hline \multicolumn{4}{|c|}{ D) Ref. 6} \\
\hline CLTO1 & $\begin{array}{l}\text { BAW-1645-4 Exp. } 2452,2.459 \% \text {-enriched } \mathrm{UO}_{2} \text { pins } \\
\text { in triangular-pitched lattice, } 1.86 \text {-cm pitch, } 435 \mathrm{ppm} \\
\text { boron }\end{array}$ & 6 & $0.9830 \pm 0.0035$ \\
\hline CLTO2 & $\begin{array}{l}\text { BAW-1645-4 Exp. } 2457,2.439 \% \text {-enriched } \mathrm{UO}_{2} \text { pins } \\
\text { in triangular-pitched lattice, } 1.86-\mathrm{cm} \text { pitch, } 335 \mathrm{ppm} \\
\text { boron }\end{array}$ & 6 & $0.9772 \pm 0.0038$ \\
\hline CLT03 & $\begin{array}{l}\text { BAW-1645-4 Exp. } 2464,2.459 \% \text {-enriched } \mathrm{UO}_{2} \text { pins } \\
\text { in triangular-pitched lattice, } 2.62-\mathrm{cm} \text { pitch, } 361 \mathrm{ppm} \\
\text { boron }\end{array}$ & 6 & $0.9818 \pm 0.0031$ \\
\hline CLT04 & $\begin{array}{l}\text { BAW-1645-4 Exp. } 2472,2.459 \% \text {-enriched } \mathrm{UO}_{2} \text { pins } \\
\text { in triangular-pitched lattice, } 3.39-\mathrm{cm} \text { pitch, } 121 \mathrm{ppm} \\
\text { boron }\end{array}$ & 6 & $0.9968 \pm 0.0043$ \\
\hline CLTO5 & $\begin{array}{l}\text { BAW-1645-4 Exp. } 2485,2.459 \% \text {-enriched } \mathrm{UO}_{2} \text { pins } \\
\text { in square-pitched lattice, } 1.778-\mathrm{cm} \text { pitch, } 886 \mathrm{ppm} \\
\text { boron }\end{array}$ & 6 & $0.9864 \pm 0.0031$ \\
\hline CLTO6 & $\begin{array}{l}\text { BAW-1645-4 Exp. } 2491,2.459 \% \text {-enriched } \mathrm{UO}_{2} \text { pins } \\
\text { in square-pitched lattice } 1.778-\mathrm{cm} \text { pitch, } 746 \mathrm{ppm} \\
\text { boron }\end{array}$ & 6 & $0.9873 \pm 0.0034$ \\
\hline CLT07 & $\begin{array}{l}\text { BAW-1645-4 Exp. } 2500,2.459 \% \text {-enriched } \mathrm{UO}_{2} \text { pins } \\
\text { in square open-pitched lattice, } 1.792-\mathrm{cm} \text { pitch, } 1156 \\
\text { ppm boron }\end{array}$ & 6 & $1.0019 \pm 0.0030$ \\
\hline CLT08 & $\begin{array}{l}\text { BAW-1645-4 Exp. } 2505,2.459 \% \text {-enriched } \mathrm{UO}_{2} \text { pins } \\
\text { in square open-pitched lattice, } 1.792-\mathrm{cm} \text { pitch, } 1068 \\
\text { ppm boron }\end{array}$ & 6 & $0.9928 \pm 0.0037$ \\
\hline
\end{tabular}


Table 2. KENO V.a calculated $k_{\text {eff }}$ for experimentally critical highly enriched uranium systems

\begin{tabular}{|c|c|c|c|}
\hline Case & Experimental description & Reference & $k_{e f r} \pm \sigma$ \\
\hline \multicolumn{4}{|c|}{ A) Ref. 5, Table 4 cases } \\
\hline CAA14 & $\begin{array}{l}\mathrm{U}(93.2) \mathrm{O}_{2}\left(\mathrm{NO}_{3}\right)_{2} \text { solution } 54.89 \mathrm{~g} \mathrm{U} / \mathrm{L}, 33.01-\mathrm{cm}- \\
\text { diam cylinder, unreflected }\end{array}$ & 28 & $1.0141 \pm 0.0039$ \\
\hline \multicolumn{4}{|c|}{ B) Ref. 5, Table 5 cases } \\
\hline $\mathrm{CASO2}$ & $\begin{array}{l}\text { Y-12 validation case A-10. } 97.67 \% \text { U metal sphere, } \\
\mathrm{H}_{2} \mathrm{O} \text { reflected }\end{array}$ & 29 & $1.0114 \pm 0.0031$ \\
\hline CASO6 & $\begin{array}{l}\text { Y-12 validation case } \mathrm{A}-4.93 .18 \% \mathrm{UO}_{2}\left(\mathrm{NO}_{3}\right)_{2} \\
\text { solution, } 20.12 \mathrm{~g} \mathrm{U} / \mathrm{L} \text {, in Al sphere, unreflected }\end{array}$ & 30 & $1.0162 \pm 0.0022$ \\
\hline $\mathrm{CAS} 07$ & $\begin{array}{l}\text { Y-12 validation case A-5. } 93.5 \% \text { U metal } \\
\text { hemispherical shell, } \mathrm{H}_{2} \mathrm{O} \text { reflected }\end{array}$ & 31 & $1.0140 \pm 0.0031$ \\
\hline CAS11 & $\begin{array}{l}\text { Y-12 validation case A-9. } 93.1 \% \mathrm{U} \text { metal } \\
\text { hemispherical shell, steel center and oil reflected }\end{array}$ & 32 & $0.9956 \pm 0.0032$ \\
\hline CAS18 & $\begin{array}{l}\text { Y-12 validation case B-15. } 92.6 \% \mathrm{UO}_{2}\left(\mathrm{NO}_{3}\right)_{2} \\
\text { solution, } 415 \mathrm{~g} \mathrm{U} / \mathrm{L} \text {, in Plexiglas cylinders, } 3 \times 3 \times 3 \\
\text { array, Plexiglas reflected (see also problem } \\
\text { U4R27G1F) }\end{array}$ & 33 & $1.0080 \pm 0.0040$ \\
\hline CAS28 & $\begin{array}{l}\text { Y-12 validation case B-8. } 93.2 \% \text { U metal cylinders } \\
\text { each in a Plexiglas box, } 2 \times 2 \times 2 \text { array of these } \\
\text { units unreflected }\end{array}$ & 34 & $1.0133 \pm 0.0031$ \\
\hline CAS32 & $\begin{array}{l}\text { Problem S333SP1. } 93.2 \% \mathrm{UO}_{2} \mathrm{~F}_{2} \text { solution, } 81.8 \mathrm{~g} \\
\mathrm{U} / \mathrm{L} \text {, in Al slabs, } 37.62-\mathrm{cm} \text { slabs in } 3 \times 1 \text { array, } \\
2.54-\mathrm{cm} \text { separation, unreflected, cylindrical tank, } \\
\text { floor, and walls in experiment room included }\end{array}$ & 35 & $0.9731 \pm 0.0034$ \\
\hline CAS52 & $\begin{array}{l}\text { Problem S63SP6. } 93.2 \% \mathrm{UO}_{2} \mathrm{~F}_{2} \text { solution, } 81.8 \mathrm{~g} \mathrm{U} / \mathrm{L} \text {, } \\
\text { in Al slabs. One slab is made up from two } 7.62-\mathrm{cm} \\
\text { slabs snugly fit together, the other is } 7.62 \mathrm{~cm}, 2 \times 1 \\
\text { array, } 15.24-\mathrm{cm} \text { separation, urreflected }\end{array}$ & 35 & $0.9883 \pm 0.0036$ \\
\hline CAS60 & $\begin{array}{l}\text { Problem U2B271F. } 92.6 \% \mathrm{UO}_{2}\left(\mathrm{NO}_{3}\right)_{2} \text { solution, } 279 \\
\mathrm{~g} \mathrm{U/L} \text {, in Plexiglas cylinders, } 3 \times 3 \times 3 \text { array, } \\
\text { unreflected, walls, floor, and tank in experiment } \\
\text { room included }\end{array}$ & 33 & $1.0027 \pm 0.0044$ \\
\hline CAS74 & $\begin{array}{l}\text { Problem U4R27I1F. } 92.6 \% \mathrm{UO}_{2}\left(\mathrm{NO}_{3}\right)_{2} \text { solution, } \\
415 \mathrm{~g} \mathrm{U} / \mathrm{L} \text {, in Plexiglas cylinders, } 3 \times 3 \times 3 \text { array, } \\
\text { reflected, } 15.24-\mathrm{cm} \text { paraffin all faces }\end{array}$ & 33 & $1.0254 \pm 0.0044$ \\
\hline
\end{tabular}




\section{DISCUSSION AND CONCLUSIONS}

The experiments modeled were parameterized such that a trend analysis could be performed. Table B.1 (Appendix B) is a relisting of the calculated results of the validation experiments. The mean $k_{\text {eff }}$ and standard deviation for the calculations are presented in Table 3, treating the data for low'enriched and highly enriched experiments separately and combined. The mean value of $k_{\text {eff }}$ is in excellent agreement with the experiments.

Table 3. Average $\mathrm{k}_{\text {eff }}$

\begin{tabular}{lccccc}
\hline & $\mathrm{N}$ & Mean & $\begin{array}{c}\text { Standard } \\
\text { deviation }\end{array}$ & $\begin{array}{c}\text { Minimum } \\
\text { value }\end{array}$ & $\begin{array}{c}\text { Maximum } \\
\text { value }\end{array}$ \\
\hline $\begin{array}{l}\text { Low enriched } \\
\text { (from Table 1) }\end{array}$ & 48 & 0.99628 & 0.00993 & 0.9772 & 1.0158 \\
$\begin{array}{l}\text { Highly enriched } \\
\text { (from Table 2) }\end{array}$ & 11 & 1.0043 & 0.01447 & 0.9731 & 1.0254 \\
Combined & 59 & 0.99778 & 0.01224 & 0.9731 & 1.0254 \\
\hline
\end{tabular}

The combined data were tested for correlation of variability to $\mathbf{k}_{\text {eff }}$. The results indicate that $k_{\text {eff }}$ is correlated to the average energy group causing fission (AEG) and to the class-variable FUEL. The variation of $k_{\text {eff }}$ with AEG is not as large if the data are separated into low-enriched and highly enriched calculations. It is thought that the variation of calculated $k_{\text {eff }}$ as a function of fuel type is related to the same factors that contribute to the variation of $k_{\text {eff }}$ with AEG.

Figures 1 and 2 present scatter plots of $k_{\text {eff }}$ as a function of AEG for low-enriched calculations, and low-enriched and highly enriched combined. Also presented in the figures are a linear-least-squares fit through the data, a linear uniform width band that encompasses the $95 \%$ confidence band for a single future calculation, and the $95 \%$ closed interval, uniform-width lower tolerance band on $99.9 \%$ proportion of the population.

For these figures, the "bias" is defined as the difference between the least-squares fit and a $\mathrm{k}_{\text {eff }}=1.0$ for the critical experiments. The general trend is that there is a positive bias of about $0.8 \%$ for the lowest AEG, decreasing to a negative bias of about $0.5 \%$ at the highest AEG.

The bias observed is consistent with two factors that affect calculations performed with 27group ENDF/B-IV library in SCALE: (1) the unresolved resonance region cross sections for ${ }^{235} \mathrm{U}$ and ${ }^{238} \mathrm{U}$ were processed at a $\sigma_{\mathrm{p}}=50,000$; and (2) few groups are in the fast range of the 27-group library. The effect of the fixed unresolved resonance region cross sections is that resonance fission for ${ }^{235} \mathrm{U}$ is too high and resonance absorption for ${ }^{238} \mathrm{U}$ is too high. The positive bias at low AEG can be attributed, in part, to few fast groups and an overestimation of ${ }^{235} \mathrm{U}$ fission for the highly enriched systems. The effect of few fast groups is that the cross sections are not independent of the collapsing spectrum used to generate them and this spectrum is not optimal for all systems. Other factors that may contribute to the observed bias include inadequacies in the base data for ENDF/B-IV cross sections. 


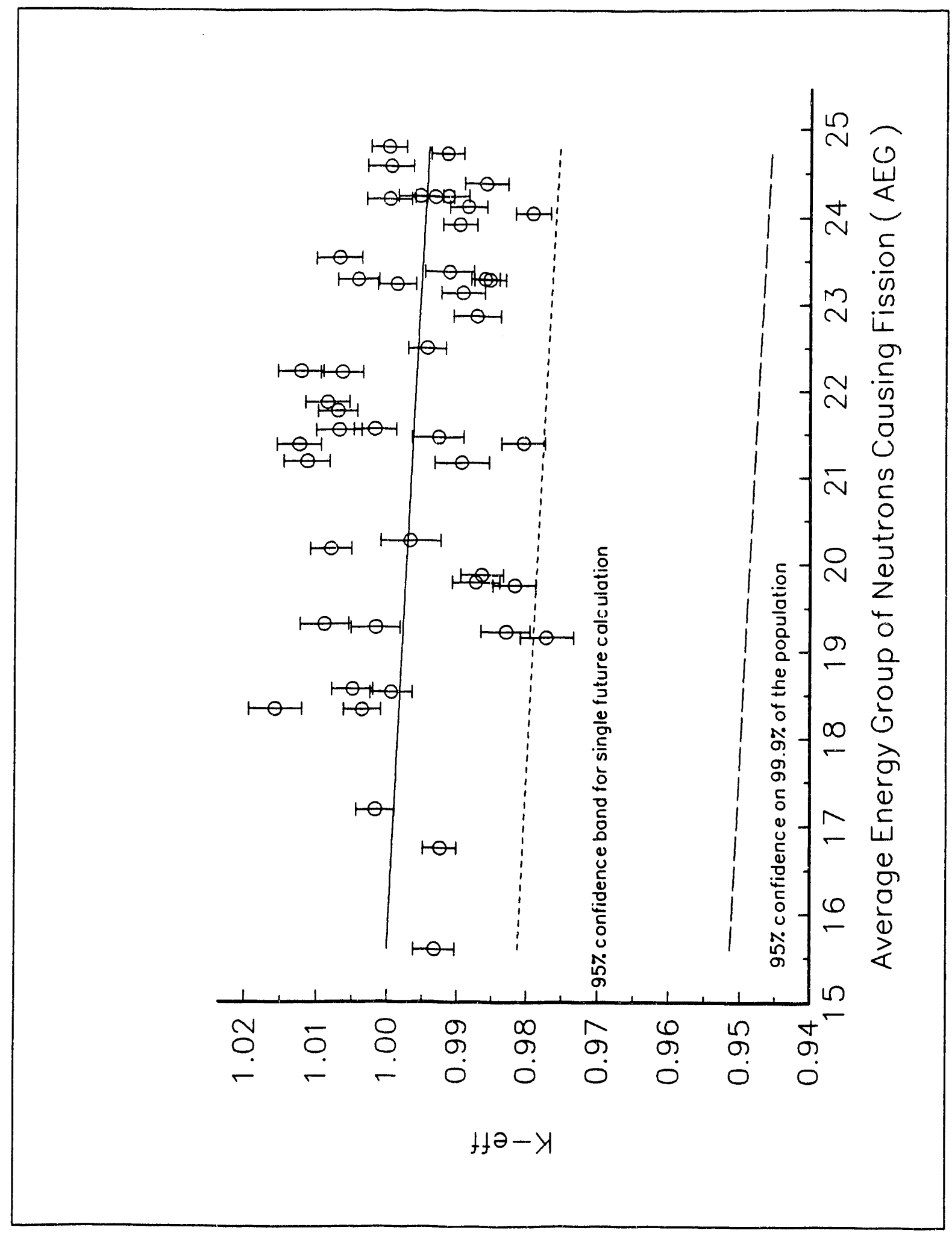




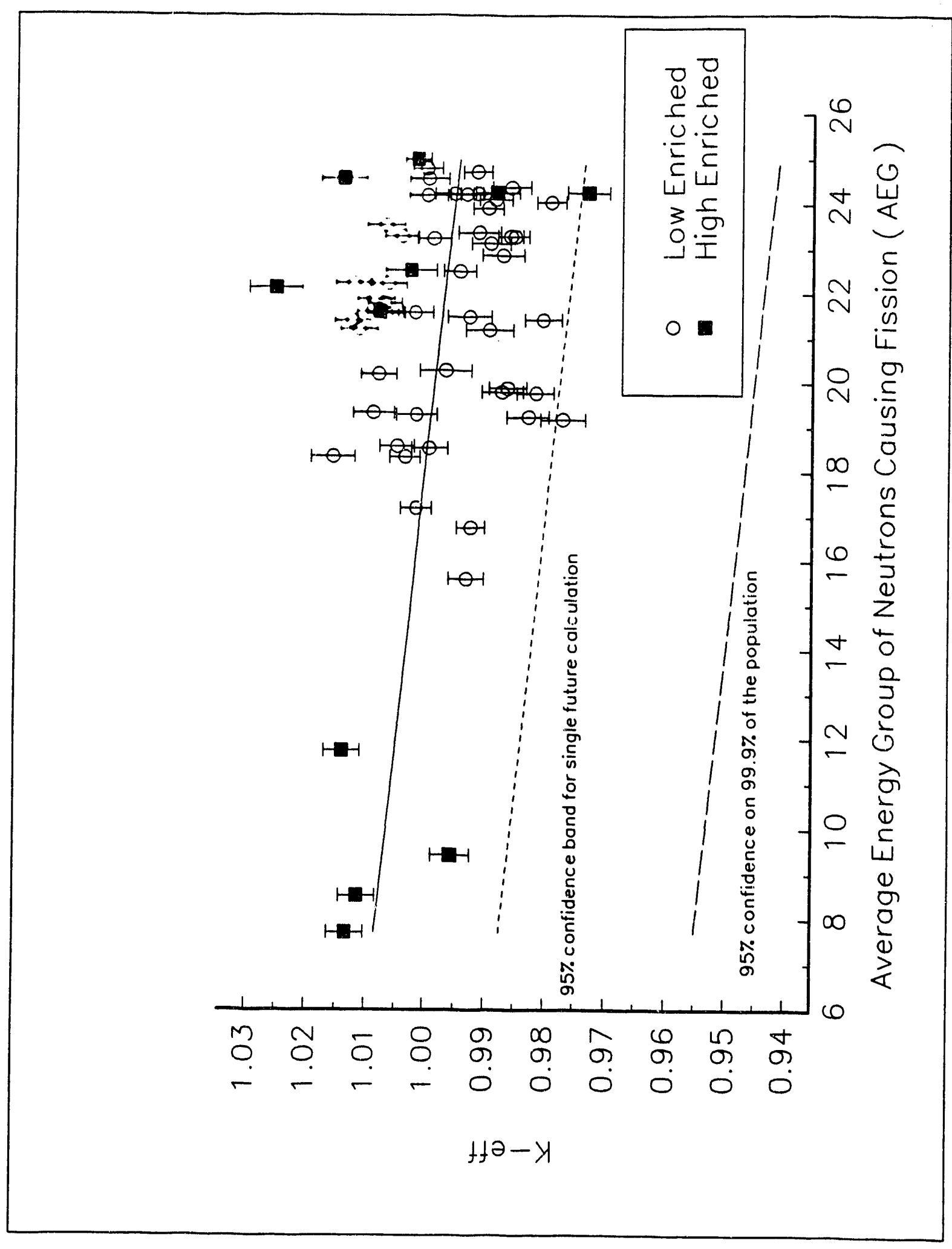

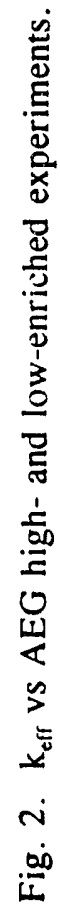


The negative bias at high AEG may be attributed, in part, to few fast groups and overestimation of the ${ }^{238} \mathrm{U}$ unresolved resonance region capture for the low-enriched systems. Not all low-enriched systems are sensitive to the unresolved resonance region cross sections. Systems that are more likely to be sensitive are undermoderated lattices and poisoned systems. The experiments in Table 1, Sect. D, exhibit a sensitivity to the unresolved resonance region processing. These are low-enriched $\mathrm{UO}_{2}$ fuel-pin-lattice experiments performed by Babcock and Wilcox Company ${ }^{36}(\mathrm{~B} \& \mathrm{~W})$ as modeled in ref. 6. A comparison of the validation results for these experiments with ref. 6 indicates that SCALE 4.0 calculates about $0.5 \%$ higher than previous versions of SCALE. This is attributed to the modifications made to the code system to more correctly treat annular geometry.

The linear uniform-width band that encompasses the $95 \%$ confidence band on a single future calculation may be interpreted as the $\mathbf{k}_{\text {eff }}$ value that, for a critical system similar to those validated, one would calculate above for a single future estimate of $k_{\text {eff }}$ at $95 \%$ confidence. Any calculated $k_{\text {eff }}$ above this band is considered critical.

The 95\% closed-interval, uniform-width, lower tolerance band on $99.9 \%$ proportion of the population is used as the calculation acceptance criteria. The values of $k_{\text {eff }}$ represented by this band may be interpreted as the $k_{\text {eff }}$ value that, for a critical system similar to those validated, one would expect to calculate above for $99.9 \%$ of the estimates of $k_{\text {eff }}$ at $95 \%$ confidence. Any calculated $k_{\text {eff }}$ below this band is considered to be of the group of systems that is not critical. The difference between the two confidence bands is taken to be the margin of calculated subcriticality. For the lowenriched systems, this margin is $0.0298 \Delta \mathrm{k}$.

Based on the results of the validation and on the statistical analysis of the data for lowenriched uranium systems, a calculated $k_{\text {eff }}$, including the uncertainty in $k_{\text {eff }}\left(k_{\text {eff }}+2 \sigma\right)$, that is less than 0.945 may be considered safely subcritical for systems similar to those validated, and provided the AEG is within the range from about 15.5 to 24.75 . The calculational limit may be increased slightly at lower values of AEG, as indicated in Fig. 1. 


\section{REFERENCES}

1. L. M. Petrie and N. F. Landers, "KENO V.a: An Improved Monte Carlo Criticality Program with Supergrouping," Sect. F11 of SCALE: A Modular Code System for Performing Standardized Computer Analyses for Licensing Evaluation, NUREG/CR-0200, Rev. 4 (ORNL/NUREG/CSD-2/R4), Vols. 1, 2, and 3 (draft February 1990). Available from Radiation Shielding Information Center as CCC-54?.

2. SCALE: A Modular Code System for Performing Standardized Computer Analyses for Licensing Evaluation, NUREG/CR-0200, Rev. 4 (ORNL/NUREG/CSD-2/R4), Vols. 1, 2, and 3 (draft February 1990). Available from Radiation Shielding Information Center as CCC-545.

3. N. F. Landers and L. M. Petrie, "CSAS4: An Enhanced Criticality Safety Analysis Module with an Optimum Pitch Search Option," Sect. C4 of SCALE: A Modular Code System for Performing Standardized Computer Analyses for Licensing Evaluation, NUREG/CR-0200, Rev. 4 (ORNL/NUREG/CSD-2/R4), Vols. 1, 2, and 3 (draft February 1990). Available from Radiation Shielding Information Center as CCC-545.

4. H. R. Dyer et al., A Technique for Code Validation for Criticality Calculations," TANSAO 63-1-464, p. 238, 1991.

5. W. C. Jordan, N. F. Landers, and L. M. Petrie, Validation of KENO V.a Comparison with Critical Experiments, ORNL/CSD/TM-238, Martin Marietta Energy Systems, Inc., Oak Ridge Natl. Lab., December 1986.

6. H. R. Dyer and R. M. Westfall, KENO V.a Validation of Fuel Pin Experiments, TANSAO 55-1-760, p. 382, 1987.

7. S. M. Bowman, Configuration Management Plan for the SCALE Code System, SCALECMP-001, Rev. 1, ORNL Internal Report issued by Nuclear Engineering Applications Department of Computing and Telecommunications Division, Oak Ridge National Laboratory, September 1990.

8. J. R. Knight, L. M. Petrie, and C. V. Parks, "Standard Composition Library," Sect. M8 of SCALE: A Modular Code System for Performing Standardized Computer Analyses for Licensing Evaluation, NUREG/CR-0200, Rev. 4 (ORNL/NUREG/CSD-2/R4), Vols. 1, 2, and 3 (draft February 1990). Available from Radiation Shielding Information Center as CCC-545.

9. N. F. Landers, L. M. Petrie, and J. A. Bucholz, "The Material Information Processor for SCALE," Sect. M7 of SCALE: A Modular Code System for Performing Standardized Computer Analyses for Licensing Evaluation, NUREG/CR-0200, Rev. 4 (ORNL/NUREG/CSD-2/R4), Vols. 1, 2, and 3 (draft February 1990). Available from Radiation Shielding Information Center as CCC-545. 
10. N. M. Greene, "BONAMI-S: Resonance Self-Shielding by the Bondarenko Method," Sect. F1 of SCALE: A Modular Code System for Performing Standardized Computer Analyses for Licensing Evaluation, NUREG/CR-0200, Rev. 4 (ORNL/NUREG/CSD-2/R4), Vols. 1, 2, and 3 (draft February 1990). Available from Radiation Shielding Information Center as CCC-545.

11. N. M. Greene, L. M. Petrie, and R. M. Westfall, "NITAWL-II: SCALE System Module for Performing Resonance Shielding and Working Library Production," Sect. F2 of SCALE: A Modular Code System for Performing Standardized Computer Analyses for Licensing Evaluation, NUREG/CR-0200, Rev. 4 (ORNL/NUREG/CSD-2/R4), Vols. 1, 2, and 3 (draft February 1990). Available from Radiation Shielding Information Center as CCC-545.

12. N. M. Greene, W. E. Ford, III, L. M. Petrie, and J. W. Arwood, AMPX-77: A Modular Code System for Generating Coupled Multigroup Neutron-Gamma Cross-Section Libraries from $E N D F / B-I V$ and/or ENDF/B-V, ORNL/CSD/TM-283, Martin Marietta Energy Systems, Inc., Oak Ridge Natl. Lab., October 1992.

13. N. M. Greene et al., "3. XLACS - Module to Produce Group-Averaged Neutron CrossSection Libraries on an AMPX Master Library," Section from AMPX-77: A Modular Code System for Generating Coupled Multigroup Neutron-Gamma Cross-Section Libraries From $E N D F / B-I V$ and/or ENDF/B-V, ORNL/CSD/TM-283, Martin Marietta Energy Systems, Inc., Oak Ridge Natl. Lab. (to be published).

14. W. C. Jordan, "SCALE Cross-Section Libraries," Sect. M4 of SCALE: A Modular Code System for Performing Standardized Computer Analyses for Licensing Evaluation, NUREG/CR-0200, Rev. 4 (ORNL/NUREG/CSD-2/R4), Vols. 1, 2, and 3 (draft February 1990). Available from Radiation Shielding Information Center as CCC-545.

15. N. M. Greene et al., "3. CORECTOL - Module to Convert Pre-AMPX-77 Master Libraries to the AMPX-77 Form," Section from AMPX-77: A Modular Code System for Generating Coupled Multigroup Neutron-Gamma Cross-Section Libraries From ENDF/B-IV and/or ENDF/B-V, ORNL/CSD/TM-283, Martin Marietta Energy Systems, Inc., Oak Ridge Natl. Lab. (to be published).

16. N. M. Greene et al., "3. PERFUME - Module to Correct Legendre Polynomial Fits to Scattering Matrices," Section from AMPX-77: A Modular Code System for Generating Coupled Multigroup Neutron-Gamma Cross-Section Libraries From ENDF/B-IV and/or $E N D F / B-V$, ORNL/CSD/TM-283, Martin Marietta Energy Systems, Inc., Oak Ridge Natl. Lab. (to be published).

17. L. M. Petrie and J. T. Thomas, Assessment of Computational Performance in Nuclear Criticality, ORNL/CSD/TM-224, Martin Marietta Energy Systems, Inc., Oak Ridge Natl. Lab., January 1985.

18. D. F. Cronin, Critical Mass Studies, Part X, ORNL-2968, Union Carbide Corp., Nucl. Div., Oak Ridge Natl. Lab., 1960. 
19. E. B. Johnson, The Criticality of Large Uranium Metal Units of Low Enrichment in U-235, ORNL-6310, Martin Marietta Energy Systems, Inc., Oak Ridge Natl. Lab. (to be published).

20. F. Abbey, comp., Handbook of Experimental Criticality Data, AHSB(s) Handbook 5, 1968.

21. S. J. Raffety and J. T. Mihalczo, Homogenized Critical Assemblies of 2 and $3 \%$ Enriched Uranium in Paraffin, Y-DR-14, Union Carbide Corp., Nucl. Div., Oak Ridge Y-12 Plant, 1969.

22. E. B. Johnson, "Effect of Steel-Water Reflectors on the Criticality of Low Enriched Uranyl Fluoride Solution," p. 47 in Neutron Physics Division Annual Progress Report for Period Ending May 31, 1968, ORNL-4280, Union Carbide Corp., Nucl. Div., Oak Ridge Natl. Lab., 1968.

23. J. W. Webster and E. B. Johnson, Criticality of a Single Unit of Aqueous Uranyl Fluoride Solution Enriched to 5\% in U-235, ORNL/TM-1195, Union Carbide Corp., Nucl. Div., Oak Ridge Natl. Lab., 1965.

24. R. E. Rothe et al., Critical Experiments with Interstitially Moderated Arrays of Low Enriched Uranium Oxide, NUREG/CR-1071, RFP-3008, U.S. Nuclear Regulatory Commission, 1980.

25. G. Tuck and I. Oh, Benchmark Critical Experiments on Low Enriched Uranium Oxide Systems with $H / U=0.77$, NUREG/CR-0674, U.S. Nuclear Regulatory Commission, 1979.

26. G. R. Goebel et al., Critical Experiments on Low-Enriched Uranium Oxide Systems with $H / U=1.25$, NUREG/CR-1653, RFP-3129, U.S. Nuclear Regulatory Commission, 1980.

27. R. E. Rothe and G. R. Goebel, Critical Experiments on Low-Enriched Uranium Oxide Systems with $H / U=2.03$, NUREG/CR-2500, RFP-3277, U.S. Nuclear Regulatory Commission, 1982.

28. R. E. Rothe, Benchmark Critical Experiments on High Enriched Uranyl Nitrate Solution Systems, NUREG/CR-0041, RFP-2710, U.S. Nuclear Regulatory Commission, 1978.

29. C. C. Byers, J. J. Koelling, G. E. Hansen, D. R. Smith, and H. R. Dyer, "Critical Measurements of a Water-Reflected Enriched Uranium Sphere," Trans. Am. Nucl. Soc. 27, 412 (1977).

30. R. Gwin and D. W. Magnuson, Nucl. Sci. Eng. 12, 364 (1962).

31. H. C. Paxton, Los Alamos Critical Mass Data, LAMS-3067, Los Alamos National Laboratory, Los Alamos, New Mexico, 1964.

32. G. Tuck, "Critical Masses of Spherical and Hemispherical Enriched Uranium Assemblies," J. Nucl. Energy 23, 663 (1969). 
33. J. T. Thomas, Critical Three-Dimensional Arrays of Neutron-Interacting Units, ORNL/TM719, Union Carbide Corp., Nucl. Div., Oak Ridge Natl. Lab., 1963.

34. J. T. Thomas, Critical Three-Dimensional Arrays of Neutron-Interacting Units, Part II U(93.2) Metal, ORNL/TM-868, Union Carbide Corp., Nucl. Div., Oak Ridge Natl. Lab., 1964.

35. J. K. Fox and L. W. Gilley, Preliminary Report of Critical Experiments in Slab Geometry, ORNL/CF-56-7-148, Union Carbide Corp., Nucl. Div., Oak Ridge Natl. Lab., 1956.

36. G. S. Hoovler et al., Critical Experiments Supporting Underwater Storage of Lightly Packed Configurations of Spent Fuel Pins, BAW-1645-4, Babcock and Wilcox Company, 1981. 


\section{APPENDIX A. CSAS25 INPUT DATA USE FOR VALIDATION}

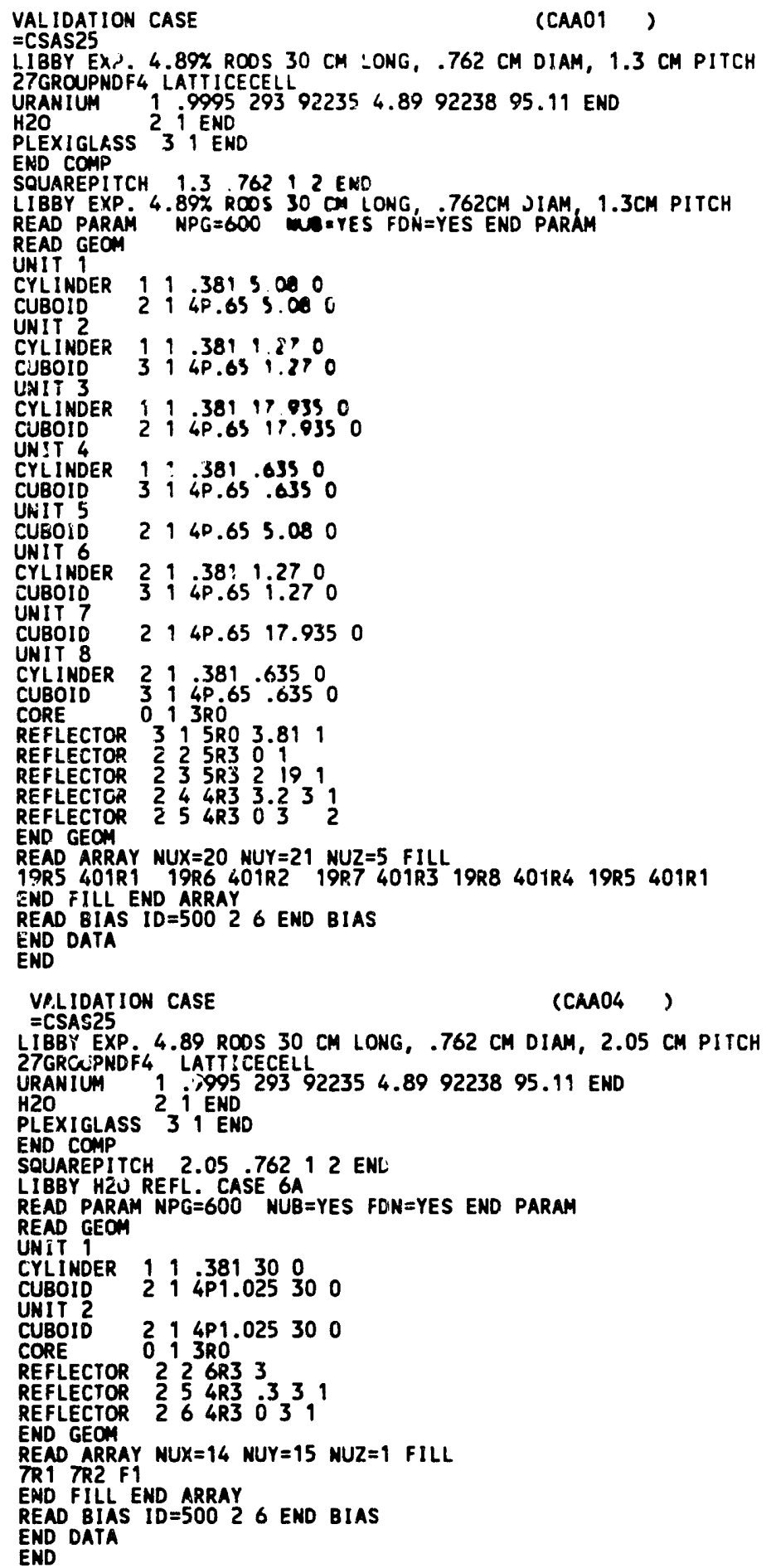


VALIDATION CASE

(CAA07)

=CSAS25

I.IBBY EXP. $4.89 \%$ ROOS 30 CM LONG, .762 CM DIAM, $1.3 \mathrm{CM}$ PITCH

27GROUPNDF4 LATTICECELL

URANIUM ${ }^{1} 9995293922354.899223895 .11$ END

H2O 2 i END

PLEXIGLASS 3

END COMP

SQUAREPITCH 1.3 .76212 END

LIBBY H2O REFL. CASE $23 B$ PB 4 FACES

READ PARAM NPG $=600$ NUB=YES PLT=NO FDN=YES END PARAM

READ GEOM

UNIT 1

CYLINDER

CUBOID

UNIT 2

CUBOID 214 P.65 270

CYI INDER

CUBOID

UNIT 8

CUBOID

UNIT 3

ARRAY $13 R 0$

REFLECTOR $214 R 3.2212 R O 1$

UNIT 9

ARRAY 4 3RO

REFLECTOR 014 L 3.221 2RO

ARRAY 53 3RO

UNIT 4

CUBOID 412 2P10.16 2P16.871 25.40

$\begin{array}{lllllll}\text { REFLECTOR } & 0 & 1 & 4 R O & 1.425 & 0 & 1 \\ \text { REFLECTOR } & 3 & 2 & 5 R O & 3.175 & 1\end{array}$

UNIT 5

ARRAY 2 3RO

UNIT 6

CUBOID 41 2P37.191 2P10.16 25.4 0

REFLECTOR 014 LRO 1.4250

REFLECTOR $325 R O 3.1751$

ARRAY $33 R O$

REFLECTOR $325 R O 3$

REFLECTOR $3 \begin{array}{lllll}6 & 5 R O & 1.97 & 1\end{array}$

END GEOM

READ ARRAY ARA $=1$ NUX $=21$ NUY $=21$ NUZ $=1$ FILL

IOR1 2 1OR1 9021 $21 R 2$ 1OR1 2 1OR1 9021

END FILL

$A R A=4$ NUX $=21 \quad N U Y=21 \quad N U Z=1 \quad F I L$

IOR7 \& 1OR7 OQ21 21R8 10R7 8 10R7 9Q21

END FIL

$A R A=2$ NUX $=3$ NUY $=1$ NUZ $=1$ FILL $410 \quad 4$ END FILL

$A R A=3$ NUX $=1$ NUY $=3$ NUZ=1 FILL 656

END FILL

$A R A=5$ NUX=1 NUY=1 NUZ=2 FILL 39

END FILL END ARRAY

READ BIAS ID $=50026$ END BIAS

READ PLOT TTL $=1 X-Y$, PIC $=M I X$

$X U L=0 \quad Y U L=75 \quad$ ZUL $=15 \quad X L R=75 \quad Y L R=0 \quad Z L R=15$

$U A X=1$ VON $=-1$ NAX $=125$ END

TTL $=1 X-Y$, PIC=UTS END

$T T L=1 \quad Y-Z$ ' PIC $=M I X$

$X U L=33$ YUL $=0 \quad Z U L=31 \quad X L R=33 \quad Y L R=75 \quad Z L R=-14.1$

VAX $=1$ WDN $=-1$ UAX $=0$ VDN=0 MAX $=125$ END

TTL $=, \quad Y-Z$, PIC=LTS END

END WOT

EN'' DATA

END

VALIDATION CASE

(CAA12)

=CSAS25

LIBBY EXP. $4.89 \%$ ROOS 30 CM LONG, .762 CM DIAM, 2.05 CM PITCH IN UO2F2 SOLN

27GROUPNDF4 LATTICECELL

URANIUM 1.9995293922354 .899223895 .11 END

SOLNUO2F2 2 295.540 1293922354.899223895 .11 END

PLEXIGLASS 31 END

AL 49 END

SOLNUOZF2 5295.5401293922354 .899223895 .11 END

END COMP

SQUAREPITCH $2.05 .762 \uparrow 2$ END

LIBBY ROOS IN UO2F2 SOLN CASE 325

READ PARAM NPG $=600$ NUB $=$ YES FDN=YES END PARAM

READ GEO

UNIT

CYLIMDER 11.3815 .080

CYLINDER 19123815.0800

Uiti 2 


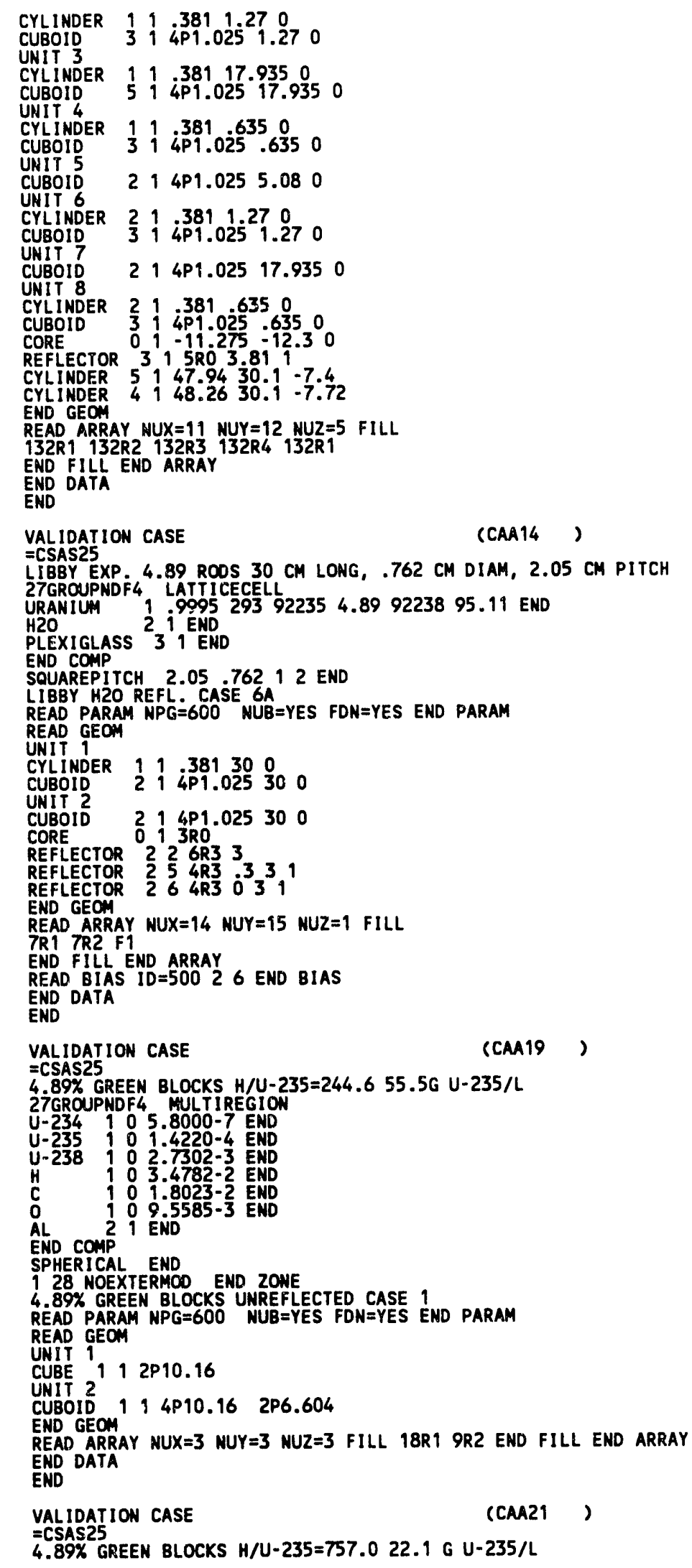




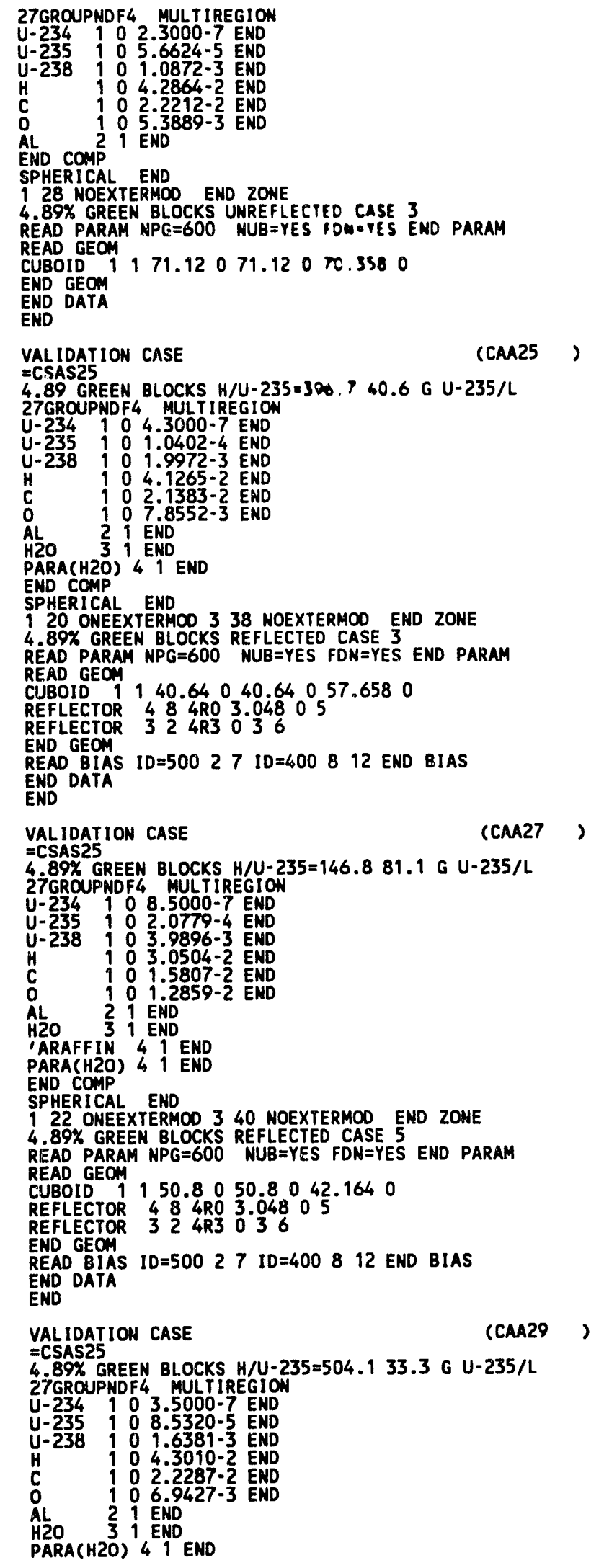




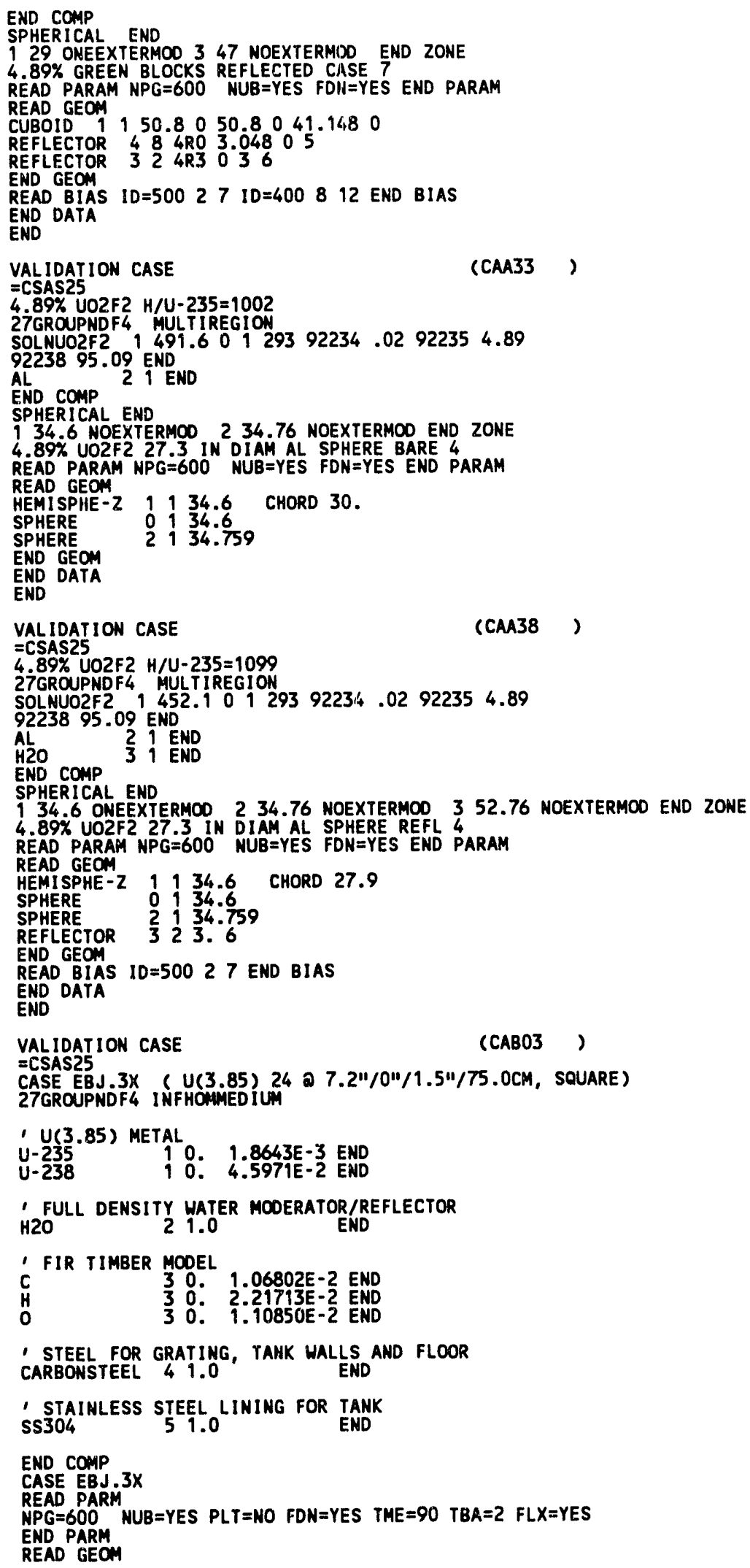


UNIT 1

COM=* FIR TIMBSR $7.2511 \times 72^{\prime \prime} \times 7.251$ *

CUBOID $3{ }^{1} 182.880$. 18.4150 . 18.4150 .

UNIT 2

COM $=\star^{2}$ WATER GAP $3.5 " \times 72 " \times 7.2511$ BETWEEN TIMBERS * CUBOID 21182.88 0. 8.89 0. 18.4150 .

UNIT 3

COM= $=$ " ARRAY OF TIMBERS 72" $\times 72 " \times 7.25 "$ PLUS $12 "$ H2O *

ARRAY 1 3*0.0

REPLICATE 211 2RO. 2RO.3175 2RO. 1

REPLICATE 214 RुO. 48 2RO. 1

UNIT 4

CON=* SECTION OF STEEL GRATING *

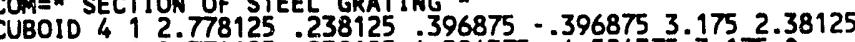

CUBOID $212.778125 .238125 \quad 4.524375-4.5243753 .1750$

CUBOID 4 i $3.016250 .24 .524375-4.5243753 .1750$.

UNIT 5

COM=* ARRAY OF STEEL GRATING PLUS $12 "$ H2O *

ARRAY $23 * 0.0$

REPLICATE $2 \uparrow 4 R 0.95252 R 0.01$

REPLICATE 2 i 4 R30.48 $2 R 0.0^{\circ} 1$

UNIT 6 COM $=*$ U(3.85) ANNULUS HITH INSERT $(75.0 \mathrm{cM} \mathrm{H})$ *

CYLINDER 113.17575 .00 .

CYLINDER 2 I 3.30275 .00

CYLINDER 199.14475 .00 .

UNIT 7

COM $=\star$ HATER CUBOID TO COMPLETE ANNULI ARRAY $(75.0 \mathrm{cM} \mathrm{H})$ * CUBOID 214 P11.049 75.00 .

UNIT 8

COM $=*$ ARRAY OF 24 ANAULI WITH U(3.85) INSERTS $(75.0 \mathrm{CM} \mathrm{H})$ *

ARRAY 3 3*0.0 4 REPLICATE 2 1 4 R36.195 2 RO $\dot{1}$

UNIT 9 COM $=*(3.85)$ ANNULUS HITH INSERT $(1.2 \mathrm{cM} \mathrm{H})$ *

CYLINDER 193.1751 .20 .

CYLINDER O 13.3021 .20 .

CYLINDER 013.3021 .20$.

CUBOID 0 i 4 P $19.049^{1} i^{2}{ }^{0} \dot{0}$

UNIT 10

COM $=*$ VOID CUBOID TO COMPLETE ANNULI ARRAY $(1.2 \mathrm{CM} \mathrm{H})$ *

CUBOID 014911.0491 .20 .

UNIT 11 COM=* ARRAY OF 24 ANNULI WITH U(3.85) INSERTS ( $1.2 \mathrm{CM} \mathrm{H}$ ) *

ARRAY $43 * 0.0$

REPLICATE 0 \% 4 R36.195 2R0.0

REPLICATE O I 4 R30.48 $2 R 0.0$ i

CORE $51-121.92-121.920$.

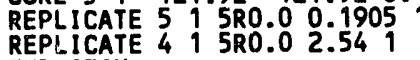

END GEOM

READ ARRAY

$A R A=1 \quad$ NUX $=1$ NUY $=13$ NUZ $=1$

FII 12502 I END FILL

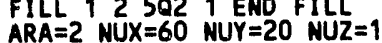

$A R A=2$ NUX $=60$ NUY

FILL F4 END FILL

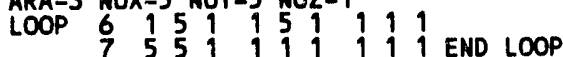

ARA $=4$ NUX $=5$ NUY $=5$ NUZ $=1$

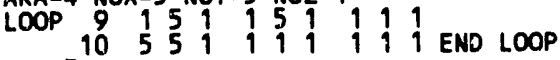

$G B L=5$

$A B L=5 \quad N U X=1 \quad$ NUY $=1 \quad$ NUZ $=4$

AILL 35811 END FILL

END ARRAY

ENDAD START

NST $=1$

$X S M=-55.245 \quad X S P=55.245 \quad Y S M=-55.245 \quad Y S P=55.245 \quad Z S M=21.59 \quad Z S P=97.79$

END START

READ PLOT 
TTL="PLAH VIEW $1 / 6$ SCALE OF PALLET, Z=MIDLEVEL OF ANNULI/INSERTS"

$X U L=-91.44$ YUL $=91.44 \quad 2 U L=59.69$
$X L R=91.44$ YLR $=-91.44$ ZLR $=59.69$

$X L R=91.44 Y L R=-91.44$
$U A X=1$. VDN $=-1 . \quad D L X=1.524$ NCH $=\star$ U.HGSC* END

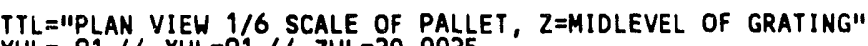

$X U L=-91.44$ YUL $=91.44 \quad Z U L=20.0025$

$X L R=91.44 \quad Y L R=-91.44 \quad Z L R=20.002$

UAX $=1$. VDN $=-1$. DLXX $=1.524$ NCH $=*$ U. WGSC* END

TTL="PLAN VIEW $1 / 6$ SCALE OF PALLET, Z=MIDLEVEL OF FIR TIMBERS"

$X U L=-91.44$ YUL $=91.44 \quad Z U L=9.2075$

$X L R=91.44 Y L R=-91.44, Z L R=9.2075$

UAX $=1$. VDN $=-1$. DLX $=1.524$ NCH $=\star$ U. HGSC* END

TTL="PLAN VIEW $1 / 10$ SCALE OF MOCKUP, $Z=M I D L E V E L$ OF $U$ UNITS"

$X U L=-137 . \quad Y U L=137 . \quad Z U L=59.69$
$X L R=137 . \quad Y L R=-137 . \quad Z L R=59.69$

$\cup A X=1$. $V D N=-1$. $D L \dot{X}=2.54$ NCH $=*$ U.WGSC* END

TIL: :"PLAN VIEW $2 X$ SCALE OF SINGLE U UNIT, $Z=M I D L E V E L$ OF UNIT"

$X U I=-11.049$ YUL $=11.049$ 2UL $=59.69$

$X L K=11.049 \quad Y L R=-11.0492 L R=59.69$

$U A X=1$. VON $=-1$. $D L X=0.127$ NCH $=\star$ U. WGSC END

TTL="CROSS SECTION OF PALLET 1/6 SCALE, $Y=0.0 "$

$X U L=-91.44$ YUL $=0.0 \quad$ ZUL $=113$

$X L R=91.44 Y L R=0.0$ ZLR=-18

UAX $=1$. WON $=-1$. DLX $=1.524$ NCH $=\star$ U. WGSC END

TTL="CROSS SECTION OF MOCKUP $1 / 10$ SCALE, $Y=0.0 "$

$X U L=-137$. YUL $=0.0 \quad Z U L=113$.

$X L R=137$ Y YLR $=0.0 \quad Z L R=-18$.
$U A X=1$. WDN $=-1 . \quad D L X=2.54$ NCH $=*$ U.WGSC* END

TTL="CROSS SECTION OF FLOOR/GRATING/FIR TIMBERS, FULL SCALE"

$X U L=0$. YUL $=0.2 U L=24.13$

$X L R=25.4 \quad Y L R=0.2 L R=-5.4$
$U A X=1 . W D N=-1 . D L X=.254 \quad \mathrm{NCH}=\star$ U. WGSC* END

TTL="PLAN VIEW OF GRATING THRU TIEBARS, FULL SCALE"

$X U L=0$. YUL $=25.4$ ZUL $=21.193$

$X L R=25, Y$ YLR=0 $2 L R=21,193$

$U A X=1$. VDN $=-1$. $D L X=.254 \mathrm{NCH}=$ * U.WGSC* END

TTL="PLAN VIEW OF GRATING Q GRATING MIDLEVEL, FULL SCALE"

$X U L=0 . \quad Y U L=25.4 \quad Z U L=20.0025$
$X L R=25.4 \quad Y L R=0$. $Z L R=20.0025$

$U A X=1$. $V O N=-1$. $O L X=.254 \mathrm{NCH}=\star$ U. WGSC* END

TTL="PLAN VIEW OF TIMBERS จ TIMBER MIDLEVEL, FULL SCALE"

$X U L=0 . \quad Y U L=25.4 \quad Z U L=9.2075$

$X L R=25.4 Y L R=-25,4$ Z $Z L R=9.2075$

$U A X=1$. VDN $=-1$. DLX $=.254 \quad N C H=\star$ U. WGSC* END

TTL="CROSS SECTION AT TOP OF UNITS, 2X SCALE"

$X U L=-11.049$ YUL $=0.2 U L L=105$.

$X L R=11.049 \quad Y L R=0 . \quad 2 L R=90$

$U A X=1$. $W D=-1$. DLX $=.127$ NCH $=*$ U. WGSC* END

END PLOT

END DATA

END

VALIDATION CASE

(CAB08)

CASE EBJ.5X ( U(3.85) 7 a $7.2 " 10 " 11.0 " 153.1 \mathrm{CM}$, TRIANGULAR )

27GROUPNDF4 INFHOMMED IUA

U U(3.85) METAL

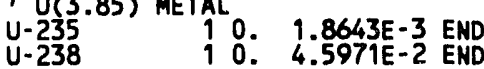

I FULL DENSITY HATER MOOERATOR/REFLECTOR

H2O 21.0 END

- fir timber model

C FIR TIMBER 30 . $1.06802 E-2$ END

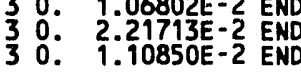

CATEEL FOR GRATING, TANK WALLS AND FLOOR

' STAINLESS STEEL. LINING FOR TANK
SS304
END 
END COMP

CASE EBJ.5X

READ PARM

$T B A=3 \quad N P G=600$ NUB $=$ YES PLT $=$ NO FDN=YES TME $=90$

READ GEOM

UNIT 9

COMIT ${ }^{9}$ FIR TIMBER $7.25 " \times 7211 \times 7.251$ *

CUBOID 31182.880 .18 .4150 .18 .4150 .

UN1T 2

COM=* HATER GAP 3.5" $\times 72 " \times 7.25 "$ BETWEEN TIMBERS *

CUBOID 2 १ 182.88 0. 8.890 . 18.4150 .

UNIT 3

COMI $=\star^{3}$ ARRAY OF TIMBERS $72 " 1 \times 72 " \times 7.25 "$ *

ARRAY $13 * 0.0$

REPLICATE 21 2RO. 2RO.3175 2RO. 1

REPLICATE 2 I $4 R 30.48$ 2RO. 1

UNIT 4

COM $=*$ SECTION OF STEEL GRATING *

CUBOID 4 个 2.778125 $.238125 \quad .396875 \quad-.3968753 .1752 .38125$

CUBOID $212.778125 .2381254 .524375-4.5243753 .1750$.

CUBOID 4 1 $3.016250 .4 .524375-4.5243753 .1750$.

UNIT 5

COM=* ARRAY OF STEEL GRATIHG *

ARRAY 2 3*0.0

REPLICATE 2 \% 1 4RO.9525 2RO.0

REPLICATE $214 R 30.482 R 0.01$

UNIT 6

COM=* $U(3.85)$ UNIT, FLOODED, $H=1.905$, LEVELS 1 AND 3 *

CYLINDER i $13.175^{\prime} 1.9050$.

CYLINDER 213.3021 .9050$.

CYLINDER 119.1441 .9050 .

UNIT 7

COM=* ARRAY OF 7 UNITS FLOODED, $H=1.905$, LEVELS 1 AND $j$ *

CUBOID 314 P91.44 1.9650 .

HOLE $60.0 \quad 0.0^{\circ} 0.0$

HOLE 6 - $20.828 \quad 0.0 \quad 0.0$

HOLE $6-10.414 \quad 18.0375770 .0$

HOLE 6 10.414 18.0375770 .0

HOLE $6 \quad 10.414-18.0375770 .0$

HOLE $6-10.414-18.0375770 .0$

REPLICATE 2 i 4 R30.48 2 RO.

UNIT 8

COM $=\star$ U(3.85) UNIT, FLOOOED $H=35.2425$, LEVEL 2 *

CYLINDER i 13.175 ' 35.24250 .

CYLINDER 213.30235 .24250$.

CYLINDER 199.14435 .24250 .

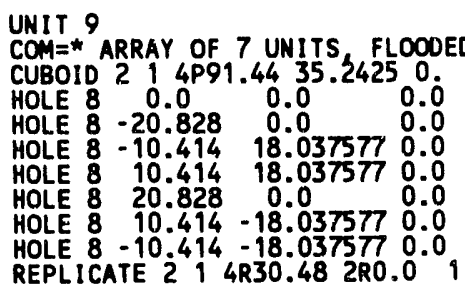

UNIT 10

COM=* U(3.85) UNIT, FLOODED $H=14.0475$, LEVEL 4 *

CYLINDER i $13.175,14.0475 \%$.

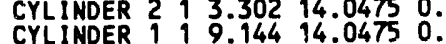

UNIT 11

COM=* ARRAY OF 7 UNITS FLOODED, $H=14.0475$, LEVEL 4 *

CUBOID 214 P99. 4414.64750

KOLE $10 \begin{array}{lll}0.0 & 0.0 & 0.0\end{array}$

HOLE $10-20.828 \quad 0.0 \quad 0.0$

HOLE $10-10.414 \quad 18.0375770 .0$

HOLE $10 \quad 10.414 \quad 18.0373770 .0$

HOLE $1020.828 \quad 0.0$

HOLE $10 \quad 10.414-18.0375770 .0$

HOLE $10-10.414-18.0375770$ 


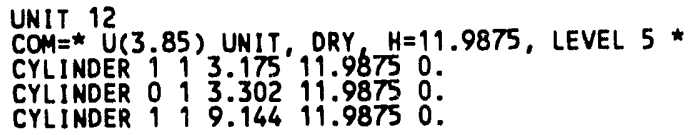

UNIT 14

COM $=* U(3.85)$ UNIT, ORY H=1.905, LEVEL 6 *

CYLINDER i $13.175,1.9050$.

CYLINDER $013.3021 .905 \%$

CYLINDER 119.1441 .9050$.

UNIT 15

COM=* ARRAY OF 7 UNITS DRY, $H=1.905$, LEVEL 6 *

CUBOID 314 PQ91.44 1.9650 .

HOLE $140.000 .0 \quad 0.0$

$\begin{array}{llll}\text { HOLE } 14 & -20.828 & 0.0 & 0.0\end{array}$

HOLE $14-10.414 \quad 18.0375770 .0$

MOLE $14 \quad 10.414 \quad 18.0375770 .0$

HOLE $14 \quad 20.828 \quad 0.077770 .0$

HOLE 14 10.414 - 18.035770 .0

HOLE $14-10.414-18.0375770^{0} i^{\circ}$

UNIT 16

COM=* U(3.85) UNIT, ORY H=9.2075, LEVEL 7 *

CYL.INDER i $13.175,9.20450$.

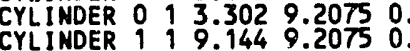

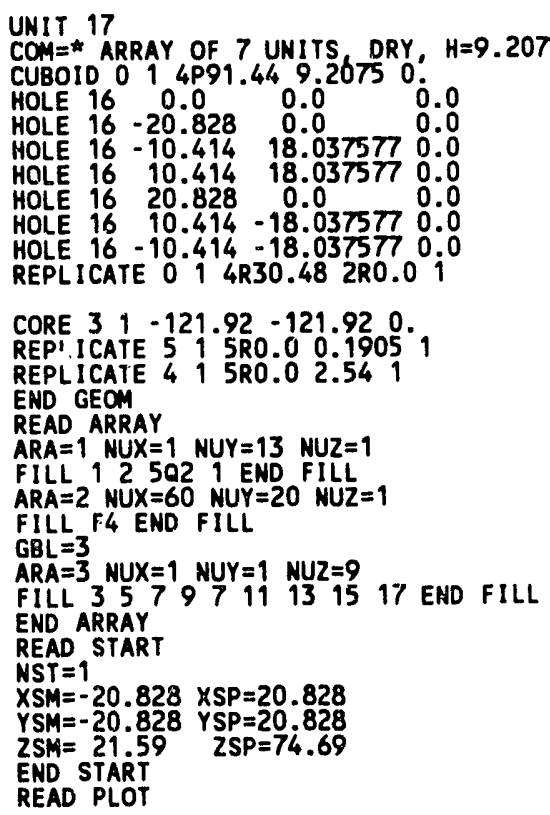

UNIT 17 CUBOID 014 P91.44 9.26750

UDO 16 0.4 .

HOLE $16-20.028 \quad 0.0 \quad 0.0$

HOLE $16-20.828 \quad 0.03770 .0$

HOLE $16-10.414 \quad 18.0375770 .0$

HOLE 16 10.414 18.0373770 .0

HOLE $16 \quad 20.828 \quad 0.0 \quad 770.0$

HOLE $16 \quad 10.414-18.0375770 .0$

REPLICATE 014 R30.48 2R0.0 $i$

CORE $31-121.92-121.920$.

REPI ICATE 5 I SRO.0 0.19051

REPLICATE 4 I 5RO.0 2.54

END GEOM

READ ARRAY

$A R A=1$ NUX $=1$ NUY $=13$ NUZ $=1$

FILL 12502 1 END FILL

ARA $=2$ NUX $=60$ NUY $=20$ NUZ $=1$

FILL F.4 END FILL

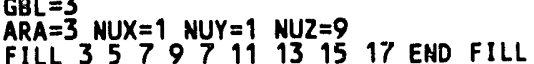

END ARRAY

END ARRAY

NST $=1$

$X S M=-20.828$ XSP $=20.828$

$Y S M=-20.828 \quad Y S P=20.828$

$2 S M=21.59 \quad 2 S P=74.69$

END START

READ PLOT

COM=* ARRAY OF 7 UNITS DRY, $H=9.2075$, LEVEL 7 *

TTL="PLAN VIEH $1 / 6$ SCALE OF CORE, Z=MIDLEVEL OF ANNULI/INSERTS"

$X U L=-100$. YUL $=100.2 U L=59.69$

$X L R=100 . Y L R=-100.2 L R=59.69$

$U A X=1$. $V D N=-1$. $D L \dot{X}=1.524 \quad M C H=\star U . W G S C *$ END

TTL="PLAN VIEW 1/6 SCALE OF CORE, Z=MIDLEVEL OF GRATIHG"

$X U L=-100$. YUL $=100$. $Z U L=20.0025$

$X L R=100, Y L R=-100, Z L R=20.0025$

UAX $=1$. VOH $=-1$. $D L \dot{X}=1.524$ NCH $=\star$ U. WGSC END

ITL="PLAN VIEH $1 / 6$ SCALE OF CORE, Z=MIDLEVEL OF FIR TIMBERS"

$X U L=-100$. YUL $=100$. $Z U L=9.2075$ 
$X L R=100 \quad Y L R=-100 . \quad 2 L R=9.2075$
$U A X=1$. VON $=-1 . \quad D L X=1.524 \quad N C H=\star$ U. WGSC* END

TTL="PLAN VIEW $1 / 10$ SCALE OF MOCKUP, $Z=M I D L E V E L$ OF $U$ UNITS" $X U L=-130 . Y U L=130.2 U L=59.69$

$X L R=130$. $Y L R=-130 . \quad Z L R=59.69$

UAX $=1$. $\dot{V} D N=-1$. DLL $\dot{X}=2.54 \quad N C H=\star$ U.WGSC* END

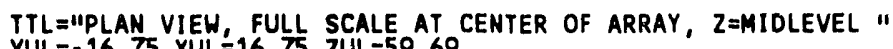
$X U L=-16.75$ YUL $=16.75$ TUL $=59.69$

$X L R=16.75$ YLR $=-16.75 \quad Z L R=59.69$

$U A X=1$. VON=-9. DLXX=0.254 NCH=* U. WGSC* END

T' $Y L=" C R O S S$ SECTION OF CORE $1 / 6$ SCALE, $Y=0.0 "$

$X U L=-100$. YUL $=0.0$ ZUL $=102$.

$U A X=1$. WDN $=-1$. DLX=1.524 NCH=* U.HGSC* END

$T T L=" C R O S S$ SECTION OF MOCKUP $1 / 10$ SCALE, $Y=0.0 "$

$X U L=-130$. YUL $=0,0$ ZUL $=105$.

$X L R=130$. $Y L R=0.0 \quad Z L R=-65$

$U A X=1$. WON $=-1$. DLX $X=2.54 \quad \mathrm{NCH}=*$ U. WGSC* END

TTL="CROSS SECTION OF GRATING/FIR TIMBERS, FULL SCALE"

$X U L=0 . \quad Y U L=0 . \quad Z U L=24.13$

$X L R=25.4$ YLR $=0$. $Z L L R=-55^{\circ}$
UAX $=1$. WON $=-1$. DLX $=.254^{\circ}$ NCH $=*$ U. WGSC* END

TIL ="PLAN VIEW OF GRATING THRU TIEBARS, FULL SCALE"

$X U L=0 . Y U L=25.4$ ZUL $=21.193$

UAX $=1$. $V D N=-1$. DLX $=.254 \quad N C H=*$ U. WGSC* END

TTL="PLAN VIEW OF GRATING \& GRATING MIDLEVEL, FULL SCALE"

$X U L=0 . \quad Y U L=25.4 \quad Z U L=20.0025$

$X L R=25.4 \quad Y L R=0 . \quad Z L R=20.0025$

UAX $=1$. VON $=-1$. DLX $=.254$ NCH $=\star$ U.HGSC* END

TTL="PLAN VIEW OF TIMBERS Q TIMBER MIDLEVEL, FULL SCALE"

$X U L=0 . \quad Y U L=25.4 \quad Z U L=9.2075$

$X L R=25.4 \quad Y L R=-25.4 Z L R=9.2075$

UAX $=1$. VDH $=-1$. DLX $=.254$ NCH $=\star$ U. WGSC* END

TTL="CROSS SECTION OF CENTRAL UNIT, $2 X$ SCALE"

$X U L=0.0$ YUL $=0.0$ ZUL $=102$.

UAX $=1$. WDN $=-1$. DLX $=0.127^{\circ}$ NCH=* U. WGSC* END

END PLOT

END DATA

END

VALIDATION CASE

(CAB11)

=CSAS25

CASE EBJ.9 U(3.85) 16 a 7.2"/2.6"/1.50"/49.2CM, SOUARE 27GROUPNDF4 INFHOMMEDIIU

( U(3.85) METAL

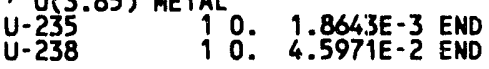

' FULL DENSITY W 1 TATER MODERATOR/REFLECTOR

- fir timber mooel

C $30.1 .06802 E-2$ END

O $30.1 .10850 E-2$ END

I STEEL FOR GRATING, TANK WALLS AND FLOOR

CARBONSTEEL 41.0 END

STAINLESS STEEL LINING FOR TANK
SS304
ENO

END COMP

CASE EBJ.9 U(3.85) 16 a $7.2 " 12.611 / 1.501 / 149.2 \mathrm{CM}$, SQUARE

READ PARM

NPG $=600$ NUB $=Y E S$ PLT $=$ NO FDN $=$ YES TME $=90 \quad T B A=2$

END PARM

END PARM

UNIT 1

COM=* FIR TIMBER $7.2511 \times 7211 \times 7.251$ *

CUBOID $31_{182.88} 0$. 18.415 0. 18.4150 . 
UNIT 2

COM $=*$ WATER GAP $3.5 " \times 72 " \times 7.25 "$ BETHEEN TIMBERS * CUBO1D 21182.880 .8 .890 . 18.4150 .

UNIT 3

COM=* ARRAY OF TIMBERS 72" $\times 72 " \times 7.25 "$ *

ARRAY 1 3*0.0

REPLICATE $212 R O$. 2RO $31732 R 0.1$

REPLICATE $214 R 30.68$ 2RO.

UNIT 4

COM=* SECTION OF STEEI COAPING *

CUBO1D \& 12.778125 .236125 $.396875-.3968753 .1752 .38125$

CUBOID 212.778125 .230185 $4.524375-4.5243753 .1750$.

CUBOID $413.016250 .+326375-4.5243753 .1750$.

UNIT 5

COM=* ARRAY OF STERI CAATIMC -

ARRAY $23 * 0.0$

REPLICATE 2 i 4 RO.OSR 200.01

COMIT ${ }^{6} U(3.85)$ ANMULUS, Ma69.2, SUBMERGED, ON CORMER *

CYLINDER 213.30260 .20

CYLINDER 2 13.30269 .200 .20

CYLINDER I 14.61413040 .20$.

CYLINDER I $15.62826469 .2 \%$.

CYLINDER I 97.24234949 .20 .

CYLINDER 117.92707949 .20

CYLINDER 118.55719949 .20

CYLINDER 199.14400049 .20

CUBOID? 14919.04949 .20 .

UNIT ${ }^{7}$ COM $\left.=* 3.85\right)$ ANNULUS, $H=49.2$, SUBMERGED, ON SIDE *

CYLINDER 213.30249 .20

CYLINDER 114.61413949 .20

CYLINDER T $4.61413949 .2 \%$.

CYLINDER I $5.62826449 .2 \%$

CYLINDER 19.46234149 .20

CYLINDER I 9.24234149 .20

CYLINDER 197.92707949 .20

CYLINDER 1 I 8.55719949 .20$.

CYLINDER 1199.14400049 .20

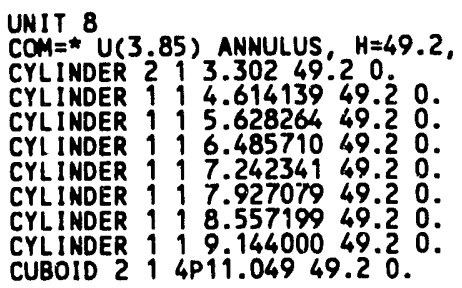

UNIT 9

COM $=* U(3.85)$ ANNULUS, $H=27.0, D R Y, O N$ CORNER *

CYLINDER 0 1 3.30227 .00

CYLINDER \& 1 i 4.61413927 .00

CYLINDER \& I 5.62826427 .00 .

CYLINDER I 5.62820427 .00

CYLINDER I 6.48571027 .00$.

CYLINDER I 17.24234927 .00

CYLINDER \& 17.92707927 .00

CYLINDER I 9.94400027 .00

CUBOID O 14 P 11.04927 .00 .

COMIT $={ }^{10} U(3.85)$ ANNULUS, $H=27.0, D R Y$, ON SIDE *

CYLINDER O 13.30227 .00 . 0 .

CYLINDER 114.61413927 .00

CYLINDER 1115.62826427 .00$.

CYLINDER I 19.48571027 .00$.

CYLINDER 117.92707927 .00

CYLINDER I I 8.55719927 .00 .

CYLINDER 119914400027.00

UNIT 11

CON=* $U(3.85)$ ANMULUS, $H=27.0, D R Y$, IN MIDDLE *

CYLINDER 013.30227 .00

CYLINDER 114.61413927 .00

CYLINDER 115.62826427 .00 . 
CYLINDER 116.48571027 .00 .

CYLINDER 117.24234127 .00 .

CYLINDER I 17.92707927 .00$.

CYLINDER if 18.55719927 .00$.

CYLINDER 119914400027.00

UNIT 12

COM=* ARRAY OF 16 ANNULI, $H=49.2$, SUBMERGED *

ARRAY $33 * 0.0$

REPLICATE 2 I 4 R47.244 2RO. 1

REPLICATE 2 i 4 R30.48 2RO. 1

UNIT 13

COM= ARRAY OF 16 ANMULI, H=27.0, DRY *

ARRAY $43^{\star 0} 0.0$

REPLICATE 014 R47.244 2RO. 1

REPLICATE 014 R30.48 2RO. 1

CORE 5 1 $1-121.92-121.920$.

REPLICATE 5 1 5 RO.0 $0.1905^{\circ} 1$

END GEOM

READ ARRAY

$A R A=1$ NUX $=1$ NUY $=13$ NUZ $=1$

FILL 12502 I END FILL

$A R A=2$ MUX 5060 NUY $=20$ NUZ $=1$

FILL F4 END FILL

$F I L L F 4$ END $F I L L$
$A R A=3$ NUX $=4$ NUY $=4 \quad$ NUZ $=1$

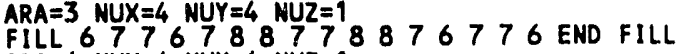

ARA $=4$ MUX $=4$ NUY $=4$ MUZ $=1 \quad 101011 \quad 11 \quad 10910109$ END FILL

$G B L=5$

$A R A=5$ NUX $=1$ NUY $=1 \quad$ NUZ $=4$

FILL 3512 13 END FILL

END ARRAY

READ START

NST $=1$

XSM $=-44.196$ XSP $=44.196$

YSM $=-44 \cdot 196 \quad Y S P=44,196$

ZSM $=21.59$ ZSP $=70.79$

END START

READ PLOT

TTL="PLAN VIEW 1/6 SCALE OF CORE, Z=MIDLEVEL OF ANHULI/INSERTS"

$X U L=-91.44 \quad Y U L=91.44 \quad Z U L=59.69$

$X L R=91.44$ YLR $=-91.44$ ZLR $=59.69$

$U A X=1$. VDN $=-1$. DLX $=1.524 \quad N C H=*$ U.WGS* END

TTL="PLAN VIEH $1 / 6$ SCALE OF CORE, Z=MIDLEVEL OF GRATING"

$X U L=-91.44$ YUL $=91.44 \quad 2 U L=20.0025$

$X L R=91.44$ YLR=-91 44 ZLR=20.0025

UAX $=1$. VON $=-1$. DLX $=1.524 \mathrm{NCH}=*$ U.HGS* END

ITL="PLAN VIEW $1 / 6$ SCALE OF CORE, $Z=M I D L E V E L$ OF FIR TIMBERS"

$X U L=-91.44$ YUL $=91.44 \quad 2 U L=9.2075$
$X L R=91.44$ YLR $=-91.44$ ZLR $=9.2075$

UAX $=1$. $V D N=-1$. DLX $=1.524$ NCH $=\star$ U. WGS* END

ITL="PLAN VIEW $1 / 10$ SCALE OF MOCKUP, $Z=$ =MIDLEVEL OF U UNITS"

$X U L=-135$. YUL $=135.2 U L=59.69$

$X L R=135$. $Y L R=-135.2 L R=59.69$

UAX $=1$. VDN $=-1$. DLL $=2.54$ NCH $=*$ U.WGS* END

TTL="PLAN VIEH $2 X$ SCALE OF SINGLE U UNIT, Z=MIDLEVEL OF UNIT"

$X U L=0.0$ YUL $=22.098 \quad$ UUL $=59.69$

$X L R=22.098$ YLR $=0$. $Z L R=59.69$
$U A X=1$. $V D N=-1$. DLX $=0.127 \quad H C H=* U . W G S^{\star}$ END

TTL="CROSS SECTION OF CORE 1/6 SCALE, $Y=11.049 "$

$X U L=-91.44$ YUL $=11.049$. $U L L=110$.

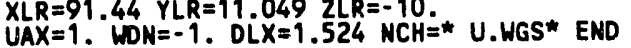

TTL="CROSS SECTION OF MOCXUP 1/10 SCALE, $Y=11.049 "$

$X U L=-135$. YUL $=11.049$ ZUL $=125$.

$X U L=-135$. YUR $=11.049 \quad 2 L R=-10$

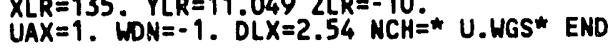

TTL="CROSS SECTION OF GRATING/FIR TIMBERS, FULL SCALE"

$X U L=0 . \quad Y U L=0 . \quad Z U L=24.13$

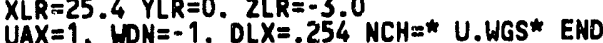

ITL="PLAN VIEH OF GRATING THRU TIEBARS, FULL SCALE"

$X U L=0$. YUL $=25.4 \quad 2 U L=21.193$
$X L R=25.4 \quad Y L R=0 . \quad Z L R=21.193$ 
$U A X=1 . V D N=-1 . \quad D L X=.254 \quad N C H=*$ U.WGS* END

TTL="PLAN VIEH OF GRATING @ GRATING MIDLEVEL, FULL SCALE" $X U L=0$.
$X I R=25,4 L=25.4 \quad Z U L=20.0025$

UAX $=1$. $V D N=-1$. DLLX $=254$ NCH=*U.WGS* END

TTL="PLLAN VIEW OF TIMBERS O TIMBER MIDLEVEL, FULL SCALE"

$X U L=0 . \quad Y U L=25.4$ ZUL $=9.2075$

$X L R=25.4 \quad Y L R=-25.4 \quad Z L R=9.2075$

$U A X=1$. VDN $=-1$. DLX $=.254$ NCH $=\star$ U. WGS* END

TTL="CROSS SECTION OF CENTRAL UNIT, FULL SCALE, Y $=11.049 "$

$X U L=0.0$ YUL $=22.098$ ZUL $=110$

$U A X=1$. $W D N=-1$. DLX $=.254$ NCH $=\star U$. HGS* END

END PLOT

END DATA

END

VALIDATION CASE

(CAB15)

$=$ CSAS25

CASE EBJ. 13 U(3.85) 15 a $2.5 " / 0.0 " / 0.75 " / 64.8 \mathrm{CM}$, SQUARE CASEROUPNDF4 INFHOMMED IUM

U U(3.85) METAL

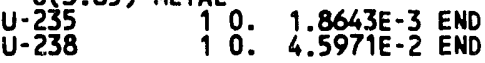

H2O FULL DENSITY 2 HATER MODERATOR/REFLECTOR

- fir timber mooel

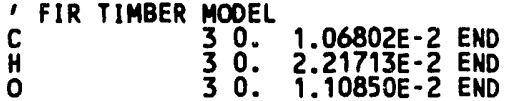

' STEEL FOR GRATING, TANK WALLS AND FLOOR

SS304

END COMP

CASE EBJ.13 U(3.85) 15 a $2.5 " / 0.0 " / 0.75 " / 64.8 \mathrm{~cm}$, SQUARE

READ PARM

NPG $=600$ NUB $=Y E S$ PLT $=$ NO FDN $=$ YES TME $=90$ TBA $=2$

END PARM

UNIT 1

COM=* FIR TIMBER 7.25" $\times 7211 \times 7.2511$ *

CUBOID 31182.880 .18 .4150 .18 .4150 .

UNIT 2

COM=" WATER GAP $3.51 \times 72 " \times 7.25 "$ BETWEEN TIMBERS *

CUBOID 2 T 182.88 0. 8.89 0. 18.4150 .

UNIT 3

COM=* ARRAY OF TIMBERS $72 " \times 72 " \times 7.251$ *

ARRAY $13 * 0.0$

REPLICATE 21 2RO. 2RO.3175 2RO. 1

REPLICATE 214 R30.48 2RO. 1

UNIT 4

COM=* SECTION OF STEEL GRATING *

CUBOID $412.778125 .238125 .396875 \cdot .396875 \quad 3.175 \quad 2.38125$

CUBOID $212.778125 .2381254 .524375-4.5243753 .1750$.

CUBOID 4 1 $3.016250 .4 .524375-4.5243753 .1750$.

UHIT 5

COM=* ARRAY OF STEEL GRATING *

ARRAY $23 * 0.0$

REPLICATE $214 R 0.9525$ 2RO.0 1

REPLICATE 2 i 4 R30.48 2RO. 1

UNIT 6

COM =* U(3.85) ROO H=64.8, SUBMERGED *

CYLINDER 111.41990364 .80 .

CYLINDER 112.00804664 .80

CYLINDER 112.45934464 .80

CYLINDER 112.83980664 .80 .

CYLINDER 113.17500064 .80 .

CUBOID 214 P4.1275 64.80 . 
UNIT 7

COM $=$ * HATER CUBOID TO COMPLETE ARRAY 3 *

CUBOID 214 P4.1275 64.8 0.

\section{UNIT 8}

COM $=* U(3.85)$ ROO $H=11.4, D R Y$ *

CYLINDER $i$ i 1.41690311 .40 .

CYLINDER I 12.00804611 .40$.

CYLINDER 192.45934411 .40$.

CYLINDER I $12.839806 \quad 11.40$.

CUBOID 014 P 4.127511 .40 .

UNIT 9

COM $=*$ VOID CUBOID TO COMPLETE ARRAY 4 *

CUBOID $O 14$ P4.1275 11.40 .

UNIT 10

COMA=* U(3.85) ROD ARRAY, H=64.8, SUBMERGED *

ARRAY $33 * 0.0$

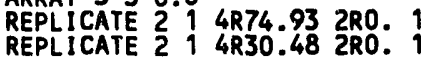

UNIT 11

COM $=\star$ ARRAY OF 15 RODS, $H=11.4$, DRY *

ARRAY $43 * 0.0$

REPLICATE 014 4R74.93 2RO.

CORE $51-121.92-121.920$.

REPLIICATE 5 I 5 RO $00.1905^{\circ}$

REPLICATE 4 I 5RO.0 2.54 1

END GEOM

READ ARRAY

$A R A=1$ NUX $=1$ NUY $=13$ NUZ $=1$

FILL 125021 END FILL

$A R A=2$ NUX $=60$ NUY $=20$ NUZ $=1$

FILL F4 END FILL

$A R A=3 \quad N U X=4 \quad N U Y=4 \quad N U Z=1$

FILL 3R6 7 12R6 END FILL

$A R A=4 \quad N U X=4 \quad N U Y=4 \quad N U Z=1$

FILL 3R8 9 12R8 END FILL

GBL $=5$

$A B A=5 \quad N U X=1 \quad$ NUY $=1 \quad N U Z=4$

ARA $=5$ NUX=1 NUY $=1$ NUZ $=4$

END ARRAY

READ START

NST $=1$

$X S M=-16.51 \quad X S P=16.51$

$Y S M=-16.51 \quad Y S P=16.5$

$Z S M=21.59 \quad Z S P=86.39$

END START

READ PLOT

TTL="PLAN VIEW 1/6 SCALE OF CORE, Z=MIDLEVEL OF ANHULI/INSERTS"

$X U L=-91.44$ YUL $=91.44$ ZUL $=59.69$

$X L R=91.44 Y L R=-91.44$ TLR=59.69

$U A X=1$. VON $=-1$. $D L X=1.524 \quad N C H=*$ U.WGS* END

TTL="PLAN VIEW FULL SCALE OF ARRAY, Z=MIDLEVEL OF UNITS"

$X U L=-16.51$ YUL $=16.51$ ZUL $=59.69$

$X L R=16.51$ YLR $=-16.51$ ZLR $=59.69$

$U A X=1$. VON $=-1$. DLX $=.254$ NCH $=*$ U.WGS* END

TTL="PLAN VIEW 1/6 SCALE OF CORE, Z=MIDLEVEL OF GRATING"

$X U L=-91.44$ YUL $=91.44 \quad Z U L=20.0025$

$X L R=91$. 44 YLR=-91.

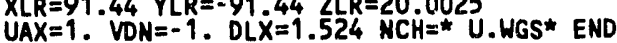

TTL="PLAN VIEW $1 / 6$ SCALE OF CORE, $Z=M I D L E V E L$ OF FIR TIMBERS"

$X U L=-91.44$ YUL $=91.44 \quad Z U L=9.2075$

$X L R=91.44 Y L R=-91.44$ ZLR $=9.207$

UAX $=1$. VON $=-1$. DLX $=1.524$ NCH $=\star$ U. WGS* END

TTL="PLAN VIEW $1 / 10$ SCALE OF MOCKUP, $Z=$ MIDLEVEL OF U UNITS"

$X U L=-135$. YUL $=135 . \quad 2 U L=59.69$

$X L R=135$. $Y L R=-135 . Z L R=59.69$

UAX $=1$. $V D N=-1$. $D L \dot{X}=2.54$ NCH $=*$ U.WGS* END

TTL="PLAN VIEW $2 X$ SCALE OF SINGLE U UNIT, Z=MIDLEVEL OF UNIT"

$X U L=0.0$ YUL $=8.255 \quad Z U L=59.69$

$X L R=8.255$ YLR $=0.0 \quad Z L R=59.69$

UAX $=1$. VON $=-1$. DLX $=0.127$ NCH=* U. WGS* END

ITL="CROSS SECTION OF CORE 1/6 SCALE, $Y=4.1275 "$

$X U L=-91.44$ YUL $=4.1275$ ZUL $=110$

$X L R=91.44 \quad Y L R=4.1275 \quad Z L R=-10$. 
UAX $=1 . \quad W D N=-1 . \quad D L X=1.524 \quad N C H=\star U . W G S^{*} E N D$

TTL="CROSS SECTION OF MOCKUP $1 / 10$ SCALE, $Y=4.12 ; j "$

$X U L=-135 . Y U L=4,1275 \quad 2 U L=125$.

$X L R=135$ Y YLR $=4.1275$ ZLR $=-10$.
$U A X=1$. WON $=-1 . \quad$ OLX $=2.54$ NCH $=*$ U.WGS* END

ITL="CROSS SECTION OF GRATING/FIR TIMBERS, FULL SCALE"

$X U L=0 . \quad Y U L=0.2 U L=24.13$
$X L R=25.4 \quad Y L R=0.2 L R=-3.0$

$U A X=1$. IDN $=-1 . \quad D L X=.254$ NCH $=$ * U. WGS* ENO

TTL="PLAN VIEH OF GRATING THRU TIEBARS, FULL SCALE"

$X U L=0 . \quad Y U L=25.4 \quad 2 U L=21.193$

UAX $=1$. VON $=-1$. DLX $=.254$ WCH $=\star$ U.HGS* END

TYL="PLAN VIEH OF GRATING a GRATING MIDLEVEL, FULL SCALE"

$X U L=0 . \quad Y U L=25.4 \quad Z U L=20.0025$

$X L R=25.4 \quad Y L R=0$. $2 L R=20.0025$

UAX $=1$. VON $=-1$. $O L X=.254$ MCH $=$ U.WGS* END

TTL="PLAN VIEW OF TIMBERS a TIMBER MIDLEVEL, FLULL SCALE"

$X U L=0$. YUL $=25.42 \mathrm{UL}=9.2075$

$X L R=25.4 Y L R=-25,4, Z 1 R=9.2075$

$U A X=1$. VON $=-1$. DiX $=.254$ MCH $=\star$ U. WGS END

TTL="CRUSS SECTION OF CENTRAL UNIT, $2 X$ SCALE, $Y=4.1275 "$

$X U L=0.0$ YUL $=4.1275$ ZUL $=110$

UAX $=1$. WDN $=-1$. DLX $=.127$ NCH=* U. HGS* END

END PIDT

END :ATA

END

VALIDATION CASE (CAR01)

ROCKY FLATS CRITICALS MUREG/CR-1071 EXPERIMENT MUMBER 1 (27 GROUP)

148 FUEL CANS 2.44 CM MOOERATOR GEE. HUT7.DATACOPTI;

48 FUEL CANS 2.44 CM MOOE

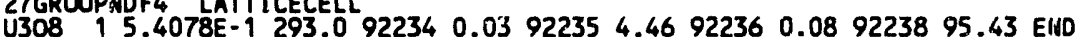

H2O $98.9514 E-2$ EMD

ARBM-BAGGIE $1.03000100114 .01601284 .980161 .2011 .9134 E-2$

END

ARBM-AL1100 $1.030011302799 .18260000 .529000 \quad 0.229 .5390 E-1$

$\begin{array}{llllllllll}\text { ARBM-TAPE(VINYL) } & 1.070 & 0 & 0 & 10015.92 & 601245.918016 & 10.82 & 17000\end{array}$ $25.73200006 .9220001 .6820009 .92919115 E-2$ END

ARBM-TAPE(MYLAR) 1.ก 3000 TÚ1 6.83601265 .50801627 .022

$1.7491 E-2$ END

ARBM-MOOERATOR 1.185300010017 .83601259 .49801632 .483 END

ARBM-PLEX(RE(i) $\quad 1.03$ h 0010017.84601259 .59801632 .234 1.1773 ENS

ARBM-PLEX(PY?ER) 1.0300010016 .48601242 .17801649 .54

ARBM-PLEX(GLUE) 1.03000100191 .67601286 .2980161 .204

$9.1648 E-3$ ENO

ARBM-PLEX(TRIS) $1.080010017 .16 \quad 601252.03 \quad 7014 \quad 0.16 \quad 801629.82$ 150311.02170001 .81350794 .260350812 .84059 .2757 END

ARBM-PLEX(PAPER) 1.0300010016 .48601242 .17801649 .55 3.7534E-3 END

ARBM-PLEX(GLUE) 1.03000100111 .67601286 .2980161 .205

ARBM-FILLER 1185300010017.83601259 .49801632 .486 .88 END

- IHIS MATERIAi IS ACTUALLY MOOERATOR ADJUSTED FOR THE VOLUME

- FRACTION OF THE VOID FILLED BETWEEN CORE AND REFLECTOR.

END COMP

SPHTRIANGP 19.946218 .58571318 .95792 END

ROCKY FLATS CRITICALS MUREG/CR-107i EXPERIMENT MUMBER 1 (27 GROUP)

148 FUEL CANS 2.44 CM MODERATOR GEE. HUT7.OATA(OPT1)

READ PARM RUN:=YES MPG $=600$

MUB $=$ YES FDN $=$ YES PLT $=$ NO

END PARM

READ GEOM

READ GEOM

COMITFIJEL BOX $15.28 \mathrm{~cm}$ OM A SIDE HITH. $15 \mathrm{~cm}$ WALLS .05CM STACKING VOID

CUBOII' 19697.49

CUBOT:D $216 P 7.64$

CUBCID $0: 6 P 7.6650$

UNIT 2

COU $=I X$-FACE INTERSTITIAL MODERATOR'

CJBOID 31 2P1.2200 4P7.665

in!? 3

COM=XY-FACE INTERSTITIAL MODERATOR.

CUBOIO 312 P7.665 2P1.2200 2P7.665 


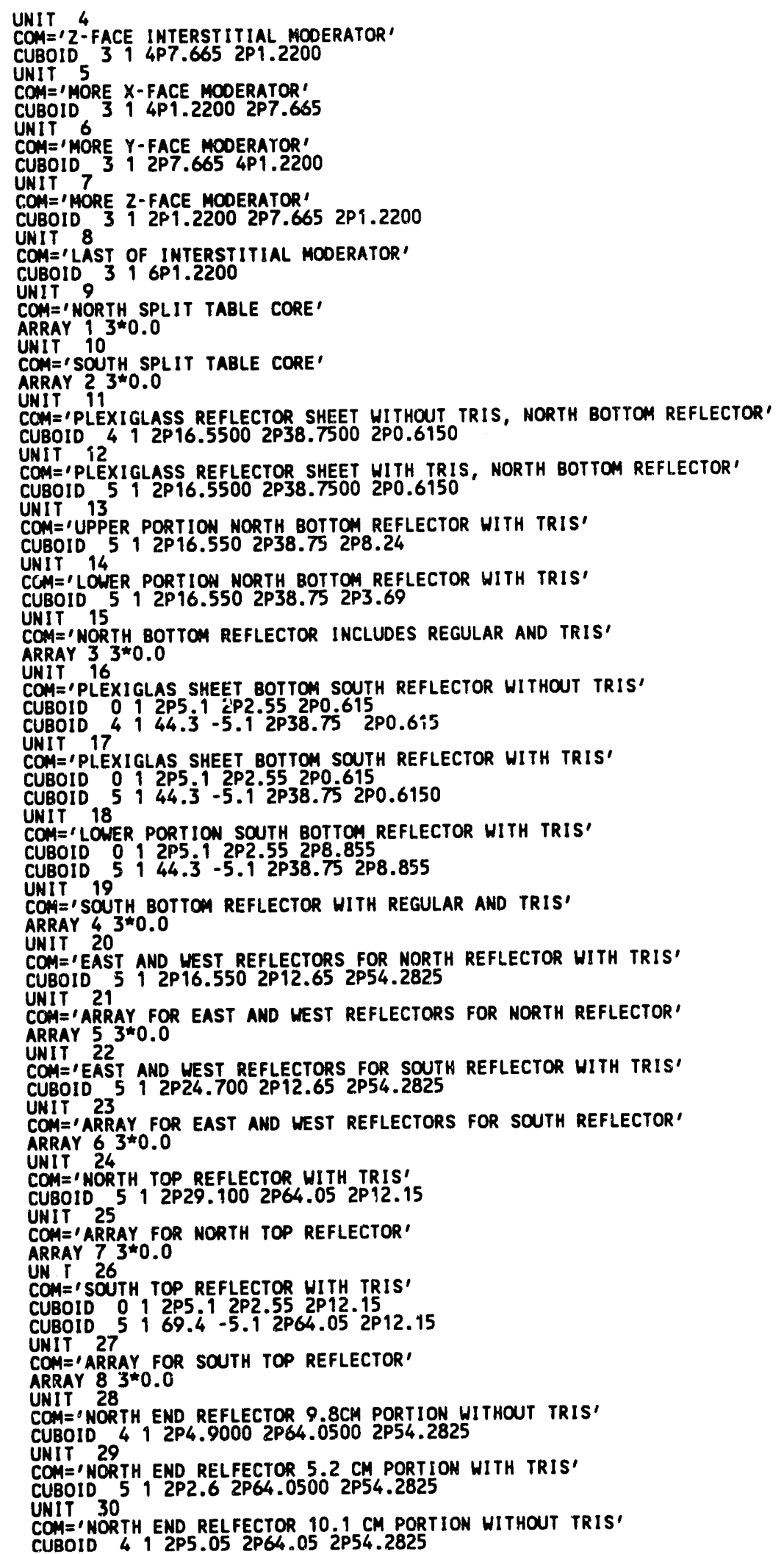




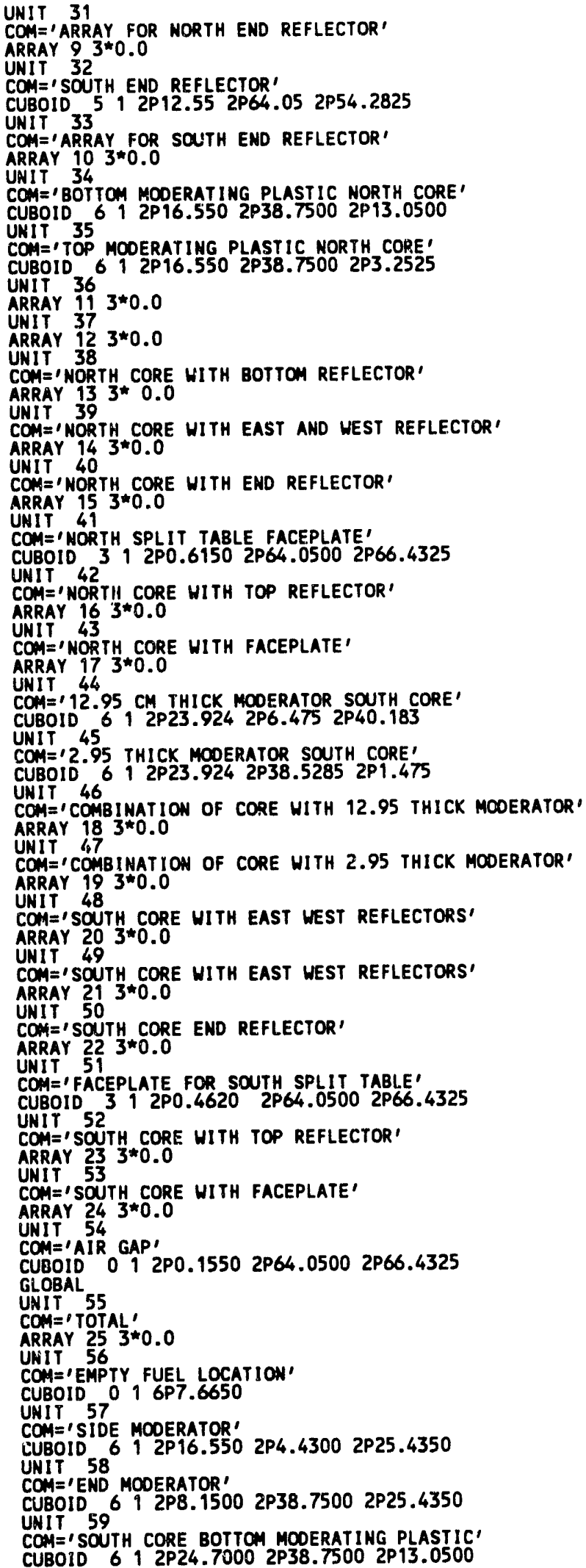




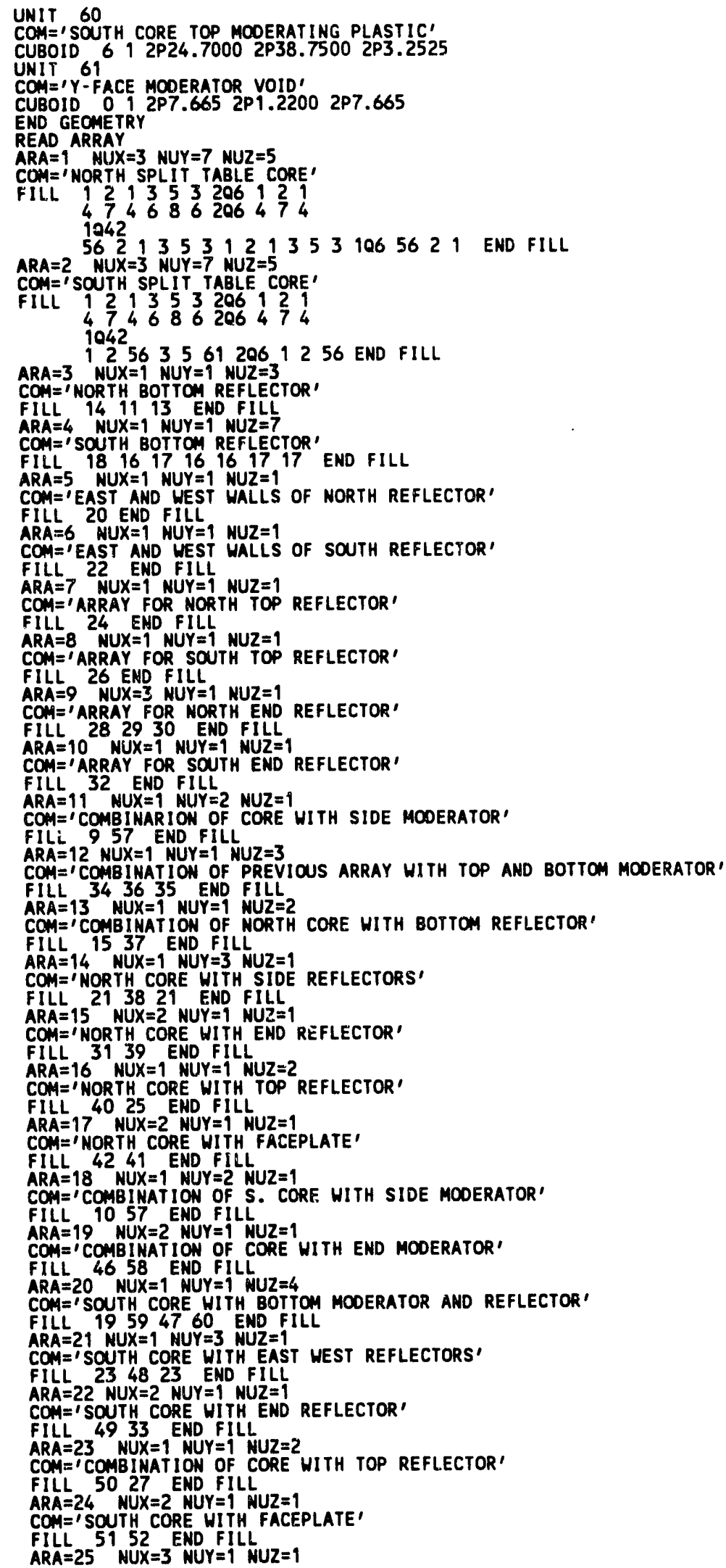




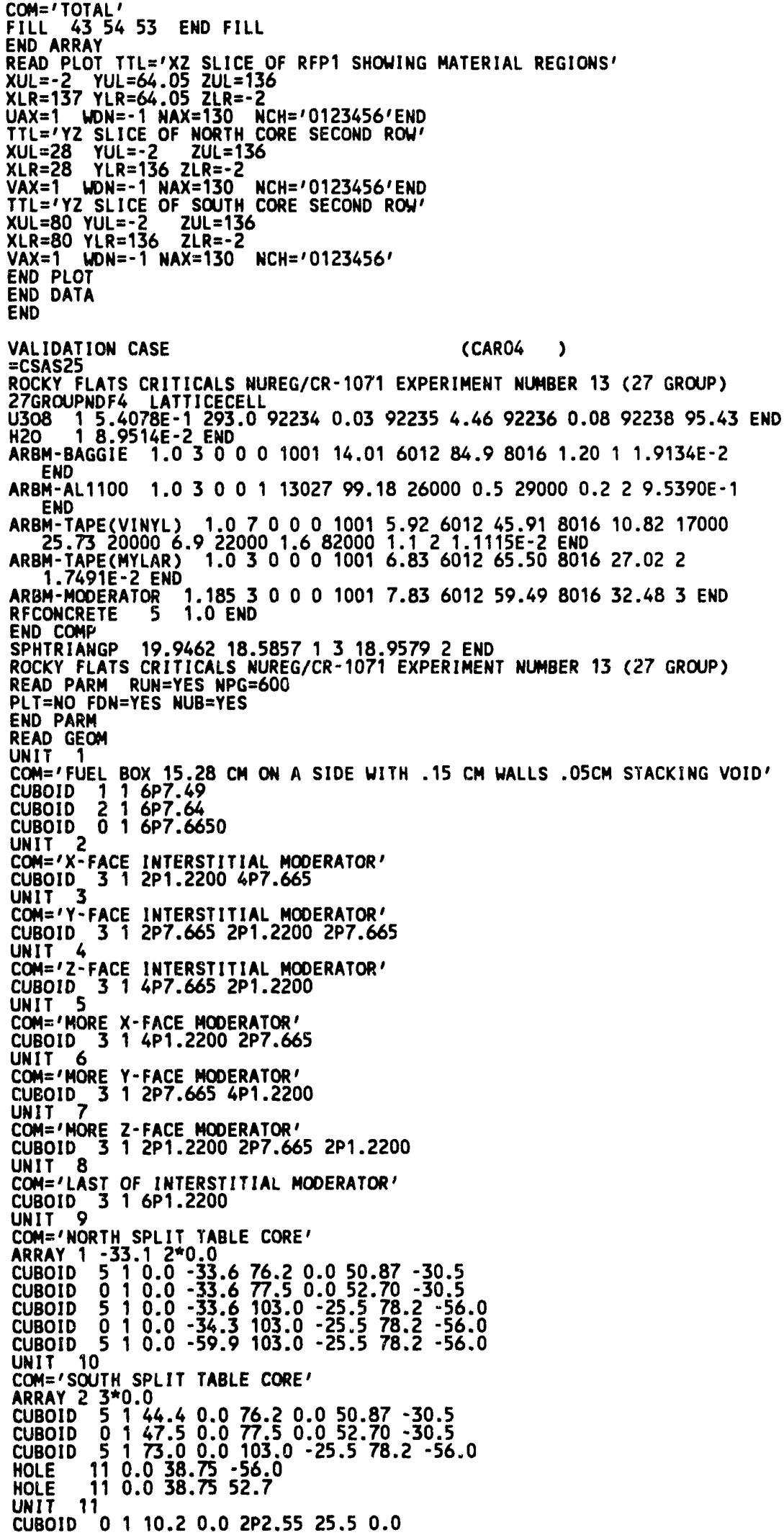




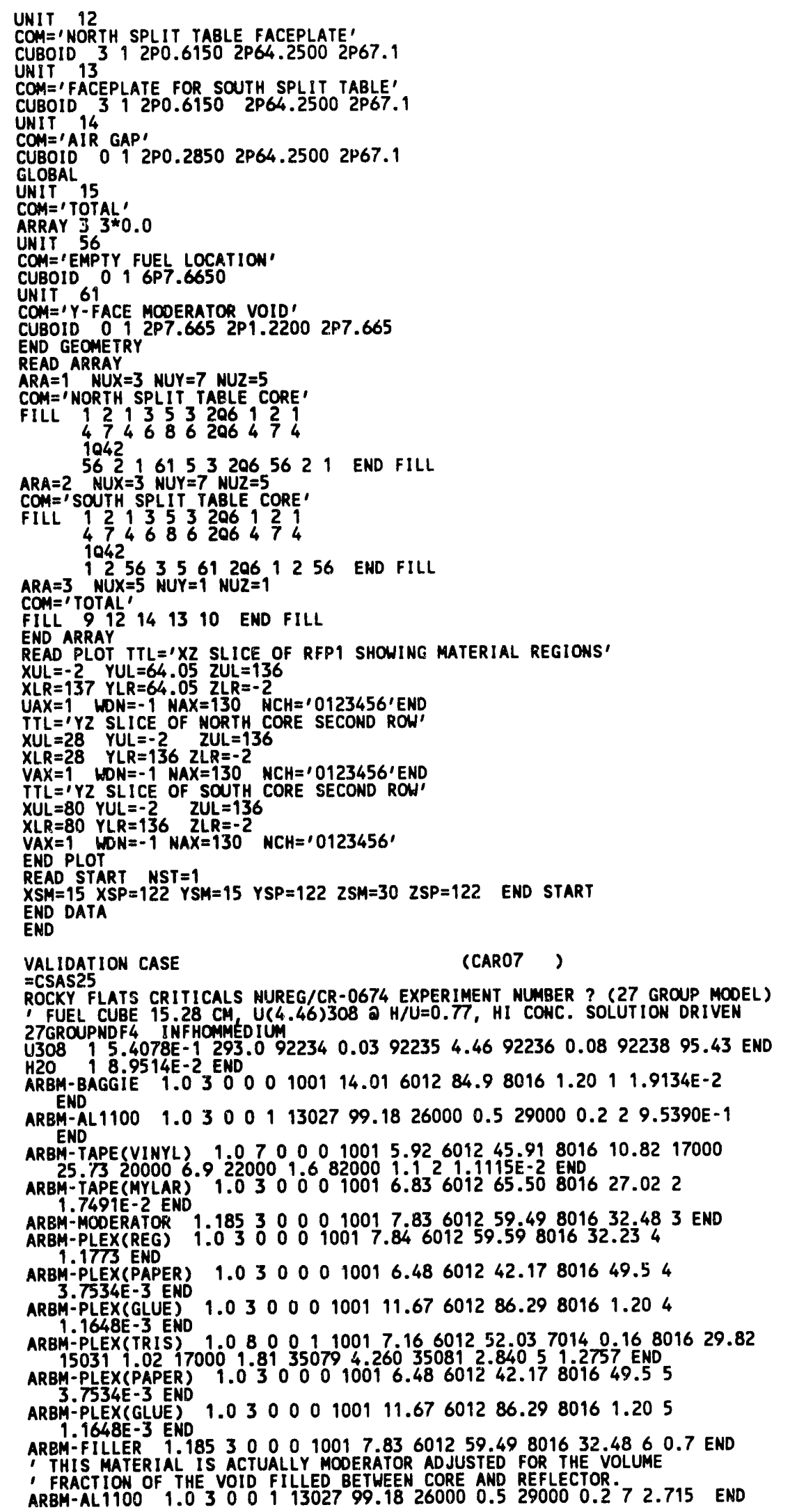




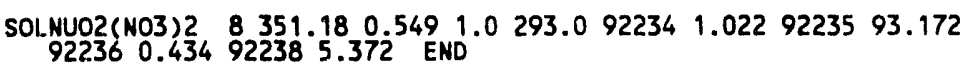

SS304 91.0 END

ROCKY FLATS CRITICALS NUREG/CR-0674 EXPERIMENT NUMBER ? (27 GROUP MOOEL)

1 FUEL CUBE $15.28 \mathrm{CM}$ U(4.46)308 a H/U $=0.77$ HI CONC. SOLUTION DRIVEN

READ PARM NPG $=600$ NÚB=YES FDN $=$ YES PLT $=$ NO ENO PARM

READ GEOM

UNIT 1

COM=' FUEL BOX WITH VOID AND PART OF AL BOX'

CUBOID $019.800 .03 .75 \quad 0.0 \quad 14.980 .0$

CUBOID $219.95 \quad 0.03 .90 \quad-0.15 \quad 14.980 .0$

CUBOID $1114.980 .0 \quad 9.365-5.61514 .980 .0$

UNIT 2

COM $=$ 'FRONT OF FUEL BOX'

CUBOID $210.150 .0 \quad 5.6150 .014 .98 \quad 0.0$

CUBOID $0190.15 \quad 0.0 \quad 9.365 \quad 0.014 .980 .0$

CUBOID $210.15 \quad 0.0 \quad 14.98 \quad 0.014 .98 \quad 0.0$

UNIT 3

COM=IREAR OF FUEL BOX

CUBOID 2 1 $0.150 .014 .98 \quad 0.014 .98 \quad 0.0$

UNIT 4

COM='ARRAY TO ASSEMBLE PART OF BOX

ARRAY 26 3*0

UNIT 5

COM=ITOP AND BOTTOM OF BOX'

$\begin{array}{llllllll}\text { CUBOID } & 0 & 9.80 & 0.0 & 3.750 & 0.0 & 0.15 & 0.0 \\ \text { CUBOID } & 2 & 15.28 & 0.0 & 9.365 & -5.615 & 0.15 & 0.0\end{array}$

GLOBAL

GLOBAL 6

COM='ARRAY TO ASSEMBLE TOP AND BOTTOM

ARRAY $273 * 0$

ARRAY 27 3*0 $15.28 \quad 0.0 \quad 15.13-0.15$

$\begin{array}{lllll}0 & 15.13 & -0.15 & 15.28 & 0.0\end{array}$

CUBOID, $0115.305-0.025 \quad 15.155-0.175 \quad 15.305-0.025$

UNIT 7 7 ROX $15.28 \mathrm{cM}$ ON A SIDE WITH .15 CM WALLS .05CM STACKING VOID'

COM=' FUEL BOX 15.28

CUBOID $216 P 7.64$

CUBOID 01 6P7.6650

UNIT 34

COM='ALUMINUM WEIGHT DISTRIBUTION PLATE FOR SPECIAL BOX'

CUBOID $019.800 .03 .750 \quad 0.0 \quad 0.160 .0$

CUBOID $7115.330 .09 .54-5.790 .160 .0$

UNIT 35

COM='ALUMINUM WEIGHT DISTRIBUTION PLATE FOR BOXES

CUBOID 714 P7.665 0.160 .0

UNIT 9

COM='NORTH SPLIT TABLE CORE'

ARRAY $13 * 0.0$

CUBOID $0130.66-0.9477 .5 \quad 0.083 .475 \quad 0.0$

UNIT 10

COM='SOUTH SPLIT TABLE CORE'

ARRAY 2 3*0.0

CUBOID $0149.4 \quad 0.077 .5 \quad 0.083 .475 \quad 0.0$

UNIT 11

COM='PLEXIGLASS REFLECTOR SHEET WITHOUT TRIS, NORTH BOTTOM REFLECTOR'

CUBOID 4 I 2P15.8000 2P38.7500 2P0.6150

UNIT 12 COM='PLEXIGLASS REFLECTOR SHEET HITH TRIS, NORTH BOTTOM REFLECTOR'

CUBO1D 512 P $15.80002 \mathrm{P} 38.75002 \mathrm{PO} .6150$

UNIT 13

COM='UPPER PORTION NORTH BOTTOM REFLECTOR WITH TRIS'

CUBOID 5 1 2 P 15.8 2P38.75 2 P8.24

UNIT 14

COM='LONER PORTION NORTH BOTTOM REFLECTOR WITH TRIS'

CUBOID 512 P 15.82 P 38.75 2P3.69

COM $=$ 'NORTH BOTTOM REFLECTOR INCLUDES REGULAR AND TRIS'

ARRAY $33 * 0.0$

ARRAY 16

COMIT 16 PLEXIGLAS SHEET BOTTOM SOUTH REFLECTOR WITHOUT TRIS

CUBOID $012 P 5.12$ P2.5 2 PO.6150

CUBOID 4 1 $44.3-5.12 P 38$. 75 2P0.615

UNIT 17

COM='PLEXIGLAS SHEET BOTTOM SOUTH REFLECTOR WITH TRIS'

CUBOID $012 P 5.12 P 2.52 P 0.6150$

CUBOID $5144.3-5.12 P 38.752 P 0.6150$

UNIT 18

COM='LOWER PORTION SOUTH BOTTOM REFLECTOR WITH TRIS'

CUBOID 0 1 2P5.1 2P2.5 2P8.855

CUBOID 5 1 $44.3-5.12 P 38$. 75 2P8.855

UNIT 19

COM='SOUTH BOTTOM REFLECTOR WITH REGULAR AND TRIS'

ARRAY $43 * 0.0$

UNIT 20

COM='EAST AND HEST REFLECTORS FOR NORTH REFLECTOR WITH TRIS'

CUBOID 5 T 2 P 15.8 2P12.65 2 P54.2825 


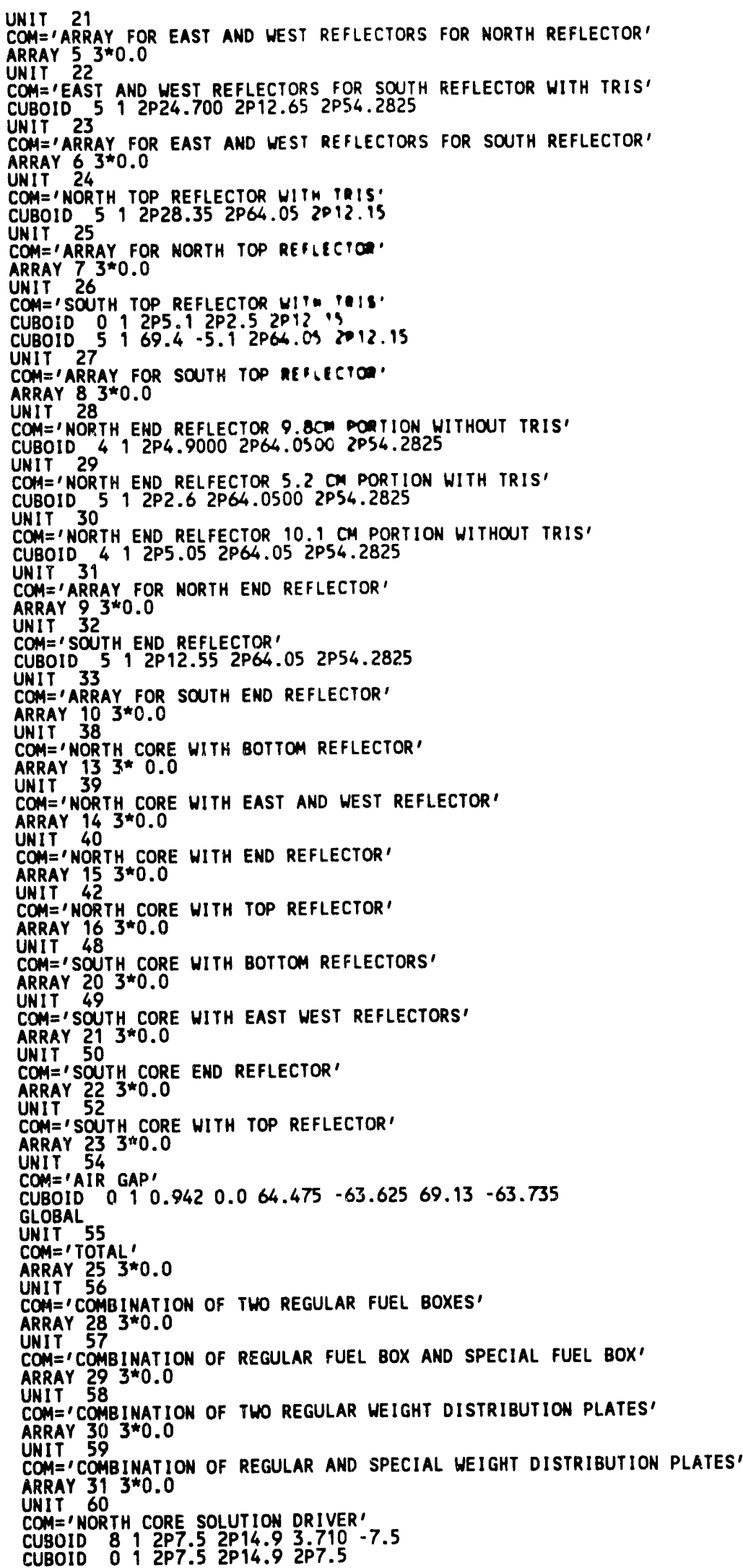




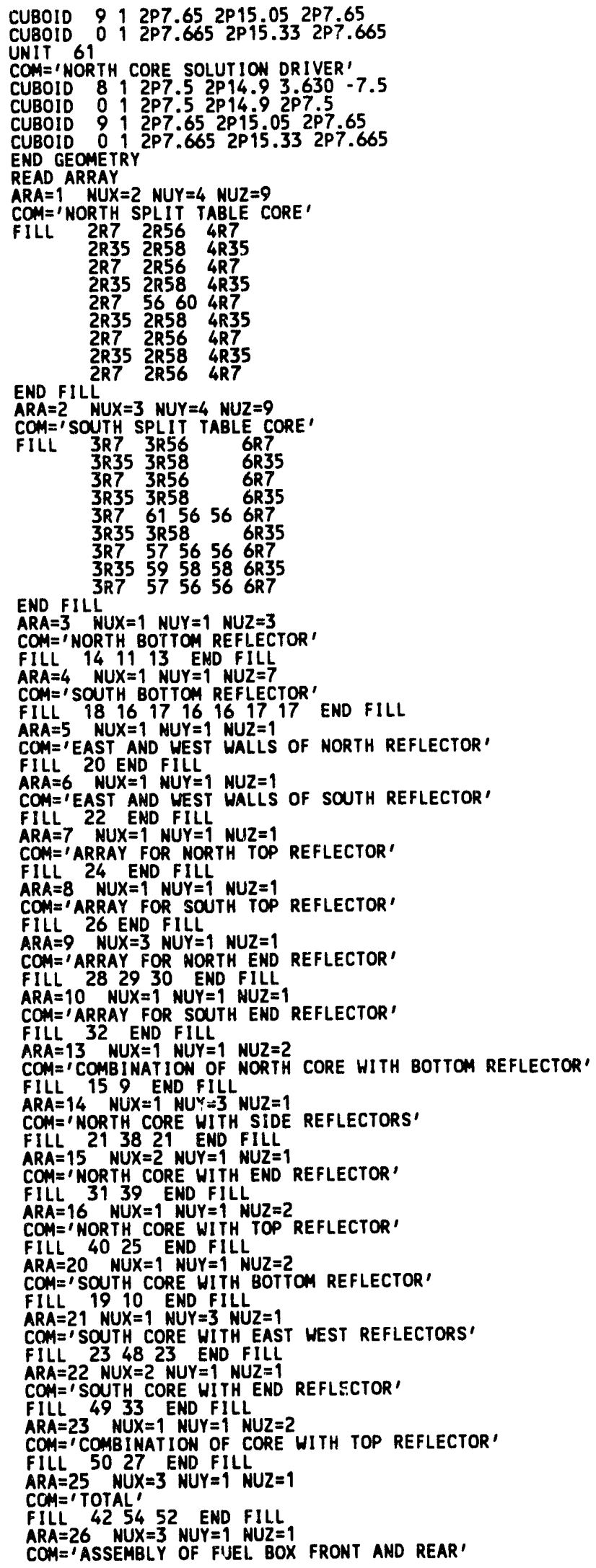




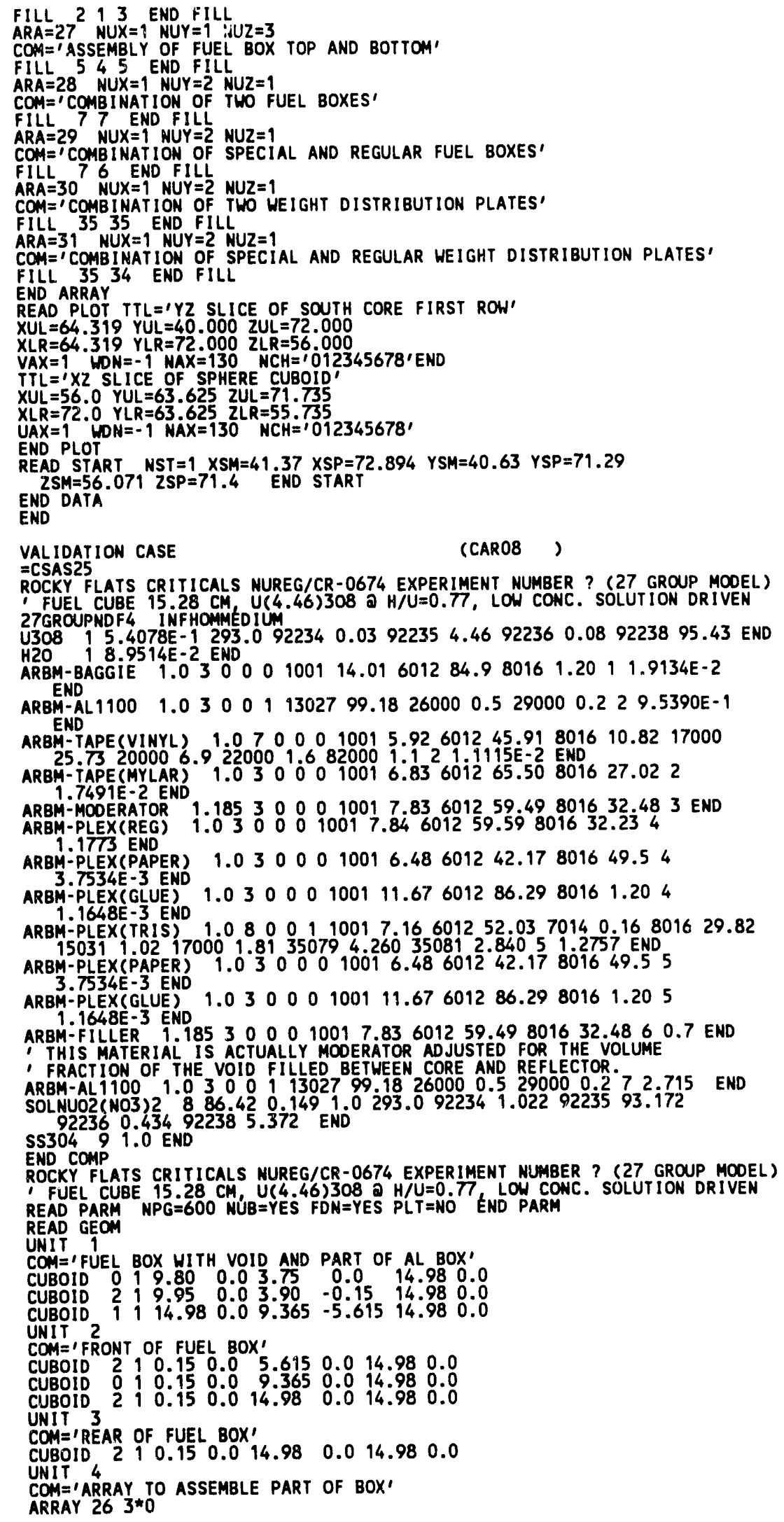


UNIT 5

COM=ITOP AND BOTTOM OF BOX'

CUBOID $019.800 .03 .750 \quad 0.0 \quad 0.150 .0$

CUBOID 2 i $15.28 \quad 0.09 .365-5.6150 .150 .0$

GLOBAL

COMIT = ARRAY TO ASSEMBLE TOP AND BOTTON'

ARRAY $273 * 0$

$\begin{array}{llllllll}\text { CUBOID } 2 & 1 & 15.28 & 0.0 & 15.13 & -0.15 & 15.28 & 0.0\end{array}$

CUBOID $0115.305-0.025 \quad 15.155-0.175 \quad 15.305-0.025$

UNIT 7

COM=IFUEL BOX $15.28 \mathrm{CM}$ ON A SIDE HITH .15 CM WALLS .05CM STACKING VOID'

CUBOID $196 P 7.49$

CUBOID $216 P 7.64$

CUBOID 0 i 6 P7.6650

UNIT 34

COMIT 34 ALINUM WEIGHT DISTRIBUTION PLATE FOR SPECIAL BOX

CUBOID $019.800 .03 .750 \quad 0.0 \quad 0.160 .0$

CUBOID 7 I 15.33 0.0 9.54 -5.790 .160 .0$

UNIT 35

COM='ALUMINUM WEIGHT DISTRIBUTION PLATE FOR BOXES'

CUBOID 714 P 7.6650 .160 .0

UNIT 9

COM=INORTH SPLIT TABLE CORE

ARRAY $13 * 0.0$

CUBOID $0130.66 \quad-0.9477 .5 \quad 0.0 \quad 83.475 \quad 0.0$

UNIT 10

COM='SOUTH SPLIT TABLE CORE'

ARRAY $233^{*} 0.0$

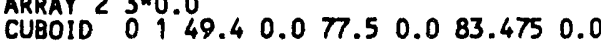

CUBOID 0149.40 .077 .50 .083 .4750 .0

COM='PLEXIGLASS REFLECTOR SHEET WITHOUT TRIS, NORTH BOTTOM REFLECTOR'

CUBOID 412 2P15.8000 2P38.7500 2P0.6150

COM='PLEXIGLASS REFLECTOR SHEET HITH TRIS, NORTH BOTTOM REFLECTOR'

CUBO1D 512 P 15.80002 2P38. 75002 2P0.6150

UNIT 13

COM='UPPER PORTION MORTH BOTTOM REFLECTOR WITH TRIS'

CUBOID 512 P15.8 2 P38.75 2P8.24

COM='LONER PORTION NORTH BOTTOM REFLECTOR UITH TRIS'

CUBOID 512 P 15.82 2P38.75 2 P3.69

UNIT 15

ARRAY $33 \div 0.0$

UNIT 16 COW='PLEXIGLAS SHEET BOTTOM SOUTH REFLECTOR WITHOUT TRIS'

CUBOID 012 P5.1 2 P2.5 2 PO .6150

CUBOID 4 T $44.3-5.12 P 38.752$ 2P0.615

UNIT 17

COM='PLEXIGLAS SHEET BOTTOM SOUTH REFLECTOR WITH TRIS'

CU8OID 0 T 2 P5.1 2 P2.5 2 P0.6150

CUBOID $5144.3-5.12 P 38$. 75 2P0.6150

UNIT 18

COM='LONER PORTION SOUTH BOTIOM REFLECTOR WITH TRIS'

CUBOID O 12 P5.1 2P2.5 2 P8.855

CUBOID 5 i $64.3-5.1$ 2P38.75 2 P8.855

UNIT 19

COM =' SOUTH BOTTOM REFLECTOR WITH REGULAR AND TRIS'

ARRAY $433^{\star 0} 0.0$

UNIT 20

COM='EAST AND WEST REFLECTORS FOR NORTH REFLECTOR WITH TRIS

CUBOID 512 P15.8 2 P12.65 2P54.2825

UNIT 21

COM='ARRAY FOR EAST AND WEST REFLECTORS FOR NORTH REFLECTOR'

ARRAY 5 3*0.0

CNIT 22 COM ='EAST AND WEST REFLECTORS FOR SOUTH REFLECTOR WITH TRIS'

COM='EAST AND WEST REFLECTORS FOR SOUI
CUBOID 5 i 2 P24.700 2 P12.65 2 P54.2825

UNIY 23

COM=IARRAY FOR EAST AND WEST REFLECTORS FOR SOUTH REFLECTOR'

ARRAY $63 * 0.0$

UNIT 24

COM=INORTH TOP REFLECTOR HITH TRIS

CUBOID 512 P28.35 2P64.05 2P12. 15

UNIT 25

COM=IARRAY FOR NORTH TOP REFLECTOR'

ARRAY $733^{*} 0.0$

COMIT 2 SOUTH TOP REFLECTOR WITH TRIS

COBOID 0 T $12 P 5.1$ RECTOR $2 P 12.15$

CUBOID $51209.4-5.12 P 64.052 P 12.15$

UNIT 27

COM='ARRAY FOR SOUTH TOP REFLECTOR'

ARRAY $83 * 0.0$ 


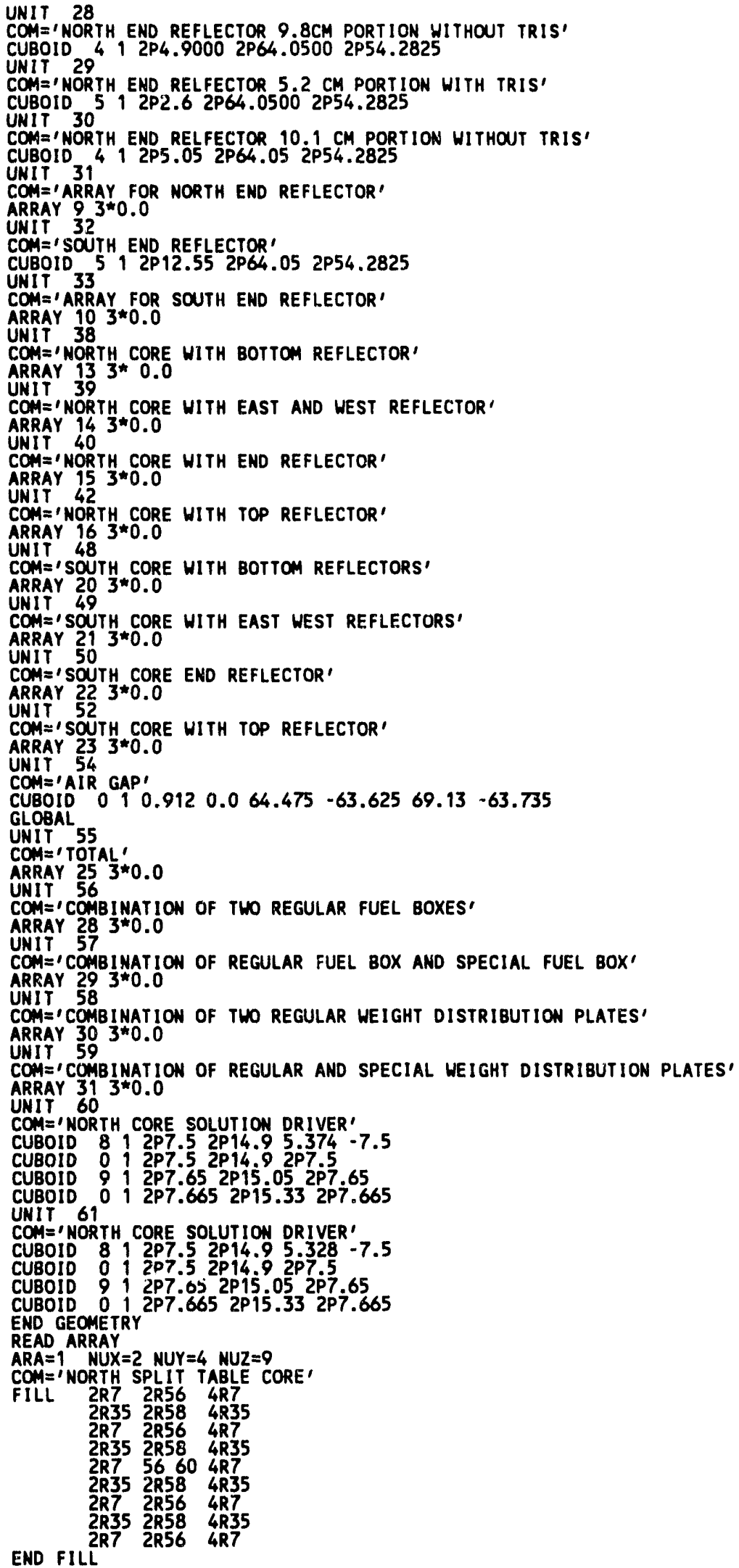




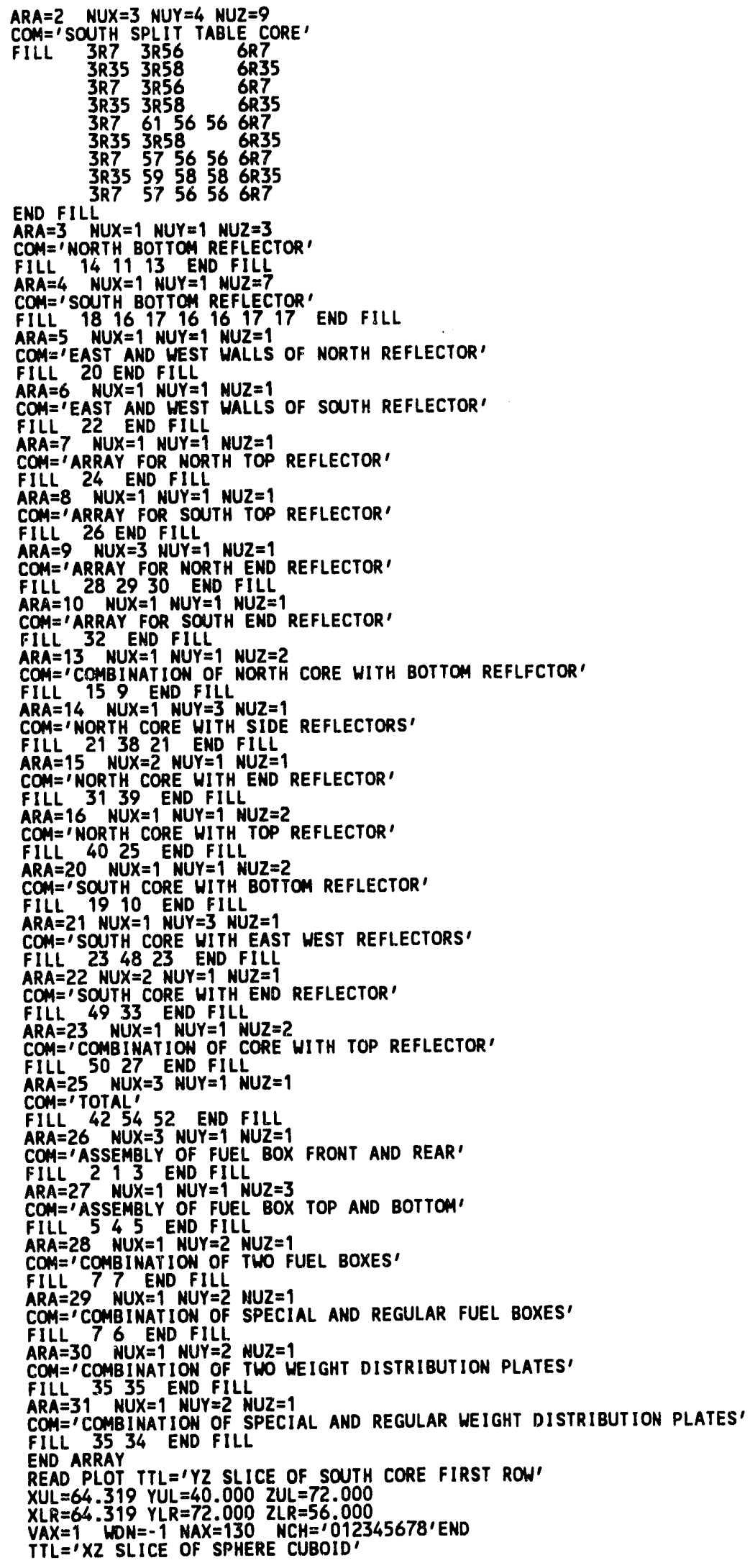


$X U L=56.0 \quad Y U L=63.625 \quad Z U L=71.735$

$X L R=72.0$ YLR=63.625 $2 L R=55.735$
$U A X=1$ WDN $=-1$ NAX $=130$ NCH $=1012345678$

UAX $=1$ WDN
END PLOT

READ START NST $=1$ XSM=41.37 XSP $=72.894$ YSM $=40.63 \quad Y S P=71.29$

ZSM $=56.071$ ZSP $=71.4$ END START

END DATA

VALIDATION CASE

(CAR11)

=CSAS25

MENT A (27 GROUP )

ROCKY FLATS CRITICALS NUREG/CR - 1653 EXPERIMENT A (27)

27GROUPNDF 4 LATIICECELL

27GROUPNDF4 LATTICECELL 922340.03922354 .46922360 .089223895 .43 END

U308 $15.4078 E-1293.0$

ARBM-BAGGIE 1.0 3 0 O $01001 \quad 14.01601284 .98016 \quad 1.2011 .9134 E-2$

END $\begin{aligned} & \text { ARBM-AL1100 } \\ & \text { END }\end{aligned} 1.0300011302799 .18260000 .5290000 .22 \quad 9.5390 \mathrm{E}-1$

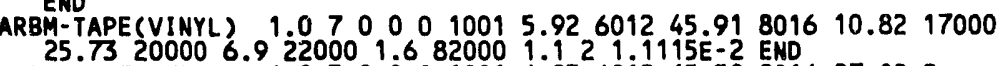

ARBM-TAPE(MYLAR) 1.0300010016 .83601265 .50801627 .022 1.7491E-2 END

ARBM-MODERATOR $1.185300010017 .83 \quad 601259.49801632 .483$ END

ARBM-PLEX(REG) $\quad 1.0300010017 .84601259 .59801632 .234$

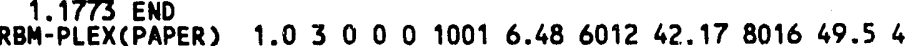

3. 7534 (PAPER)

ARBM-PLEX(GLUE) 1.03000100111 .67601286 .2980161 .204

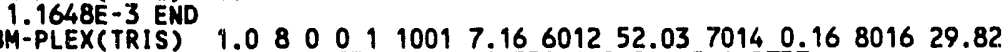

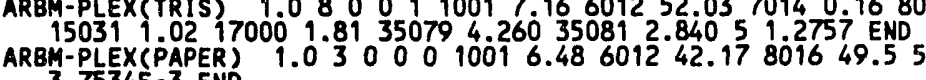

3.7534E-3 END

ARBM-PLEX(GLUE) 1.03000100111 .67601286 .2980161 .205

ARBM-FILLER $1.18530000010017 .83601259 .498016 \quad 32.486 .888$ END

- THIS MATERIAL IS ACTUALLY MODERATOR ADJUSTED FOR THE VOLUME

- FRACTION OF THE VOID FILLED BETWEEN CORE AND REFLECTOR.

SPHTRIANGP $19.9462 \quad 18.58571318 .95792$ END

ROCKY FLATS CRITICALS NUREG/CR-1653 EXPERIMENT A (27 GROUP)

148 FUEL CANS 2 44 CM MODERATOR GEE HUT25. DATACOPT

READ PARM RUN=YES NPG $=600$ FDN=YES NUB=YES PLT=NO END PARM

READ GEOM

UNIT 1

COM='FUEL BOX $15.28 \mathrm{CM}$ ON A SIDE WITH .15 CM WALLS .05CM STACKING VOID

CUBOID 1 1 6 P 7.49

CUBOID 2 I 6 6P7.64

UNIT 5

COM $=\prime X$-FACE INTERSTITIAL MOOERATOR'

CUBOID $312 \mathrm{P} 1.22004$ P7.665

UNIT 3

COM= $Y$-FACE INTERSTITIAL MOOERATOR'

CUBOID 312 P7.665 2P1.2200 2P7.665

UNIT 4

COM $=I Z$-FACE INTERSTITIAL MOOERATOR'

CUBOID 314 P7.665 2P1.2200

UNIT 5

COM $=$ 'MORE X-FACE MOOERATOR'

CUBOID 3 T 4P1.2200 2P7.665

UNIT 6

COM $=$ MORE $Y$-FACE MODERATOR'

CUBOID 31 2P7.665 4P1.2200

UNIT 7

COM=1MORE Z-FACE MOOERATOR!

CUBOID 31 2P1.2200 2P7.665 2P1.2200

UNIT 8

CUBOID 3 T 6 P 9.2200

UNIT 9

COM='NORTH SPLIT TABLE CORE'

ARRAY $13 \neq 0.0$

UNIT 10

COM =' SOUTH SPLIT TABLE CORE'

ARRAY $233^{\star} 0.0$

UNIT 11

COM='PLEXIGLASS REFLECTOR SHEET WITHOUT TRIS, NORTH BOTYOM REFLECTOR'

CUBOID 412 P 16.55002 2P38.7500 2P0.6150

UNIT 12 COM='PLEXIGLASS REFLECTOR SHEET HITH TRIS, NORTH BOTTOM REFLECTOR'

CUBOID $13^{5}$

COM= IUPPER PORTION NORTH BOTTOM REFLECTOR WITH TRIS'

CUBOID 5,12 P 16.5502 P 38.752 P 8.24 


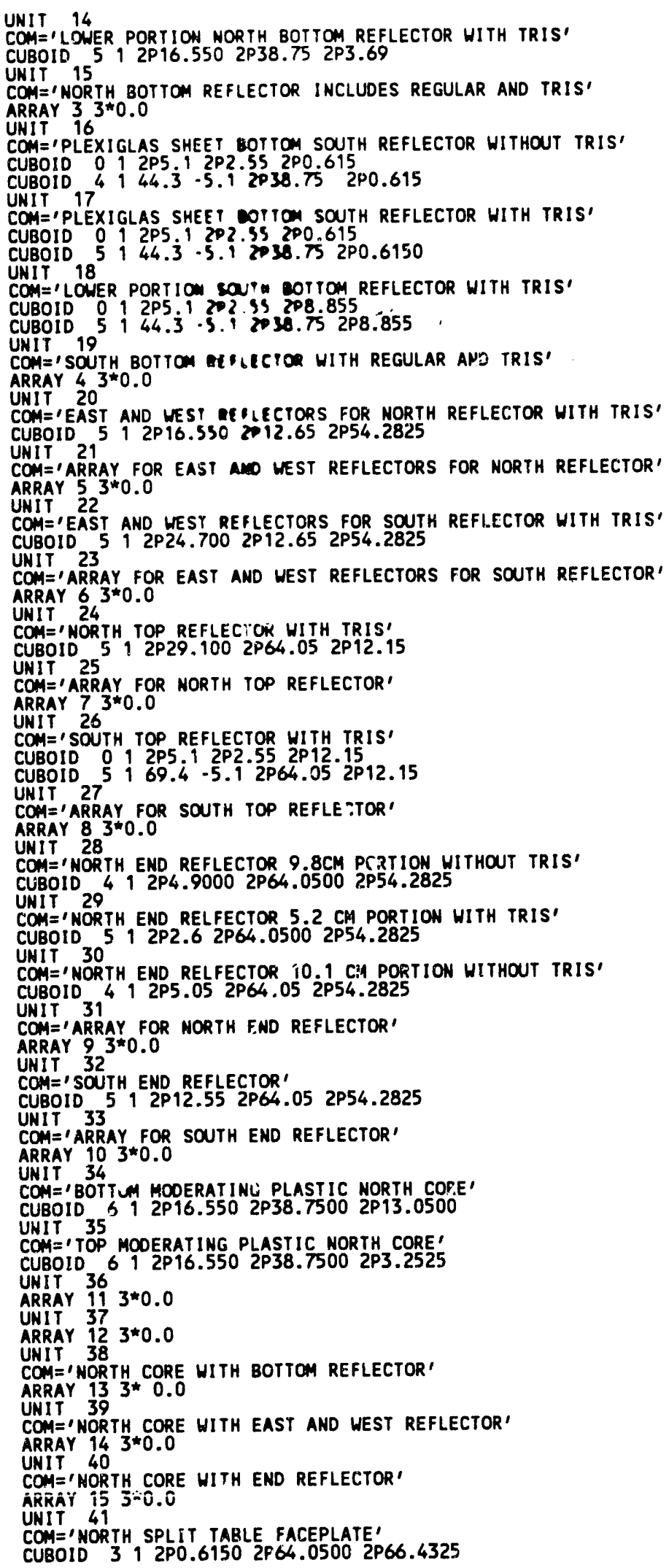


UNIT 42

COM ='NORTH CORE WITH TOP REFLECTOR'

ARRAY $163 * 0.0$

UNIT 43

FACEPLATE'

ARRAY 17 3*0.0

UNIT 44

COM='12.95 CM THICK MODERATOR SOUTH CORE'

CUBOID 61 ?P23.924 2P6.475 2 P40.183

UNIT 45

COM $=12.95$ THICK MODERATOR SOUTH COREI

CUBO1D 612 P23.924 2 P38.5285 2 P 1.475

UNIT $46^{6}$

COM=I COMBINATION OF CORE WITH 12.95 THICK MODERATOR'

ARRAY $18 \quad 3 * 0.0$

UNIT 47 34.

ARRAY $193^{* 0}$.0

UNIT 48

COM='SOUTH CORE WITH EAST HEST REFLECTORS'

ARRAY $203 * 0.0$

UNIT 49

COM $=$ ' SOUTH CORE WITH EAST WEST REFLECTORS'

ARRAY $213 * 0.0$

UNIT 50

COM=' SOUTH CORE END REFLECTOR'

ARRAY $223 \approx 0.0$

UNIT ST

CUBOID 312 PO. $4620 \quad 2 \mathrm{P} 64.0500$ 2P66.4325

UNIT 52 2P0.4620 2P64.0500 2P66.4

COM='SOUTH CORE WITH TOP REFLECTOR'

ARRAY $233 * 0.0$

UNIT 53

COM=' SOUTH CORE HITH FACEPLATE'

ARRAY $243 * 0.0$

UNIT 54

COM=IAIR GAP

CUBOID 0 Y 2P0.1550 2P64.0500 2P66.4325

GLOBAL

UNIT 55

COM $=$ ITOTAL,

ARRAY 25 3*0.0

UNIT 56

COM $=$ EMPTY FUEL LOCATION'

COM=I EMPTY FUEL LOCAT
CUBOID O 1 6P7.6650

UNIT 57

COM='SIDE MOOERATOR'

CUBOID 51 2P16.550 2P4.4300 2P25.4350

UNIT 58

COM=IEHO MODERATOR

CUBO1D 61 2P8.1500 2P38. $55002 P 25.4350$

UNIT $59^{\circ}$

COH='SOUTH CORE BOTTOH MOOEPATING PLASTIC

CUBOID 612 P24.7000 2P38.7500 2P13.0500

UNIT 60

COM=1 SOUTH CORE TOP MODERATIMG PLASTIC'

CUBOID 6 1 2 P24.7000 2P38.7500 2P3.2525

UNIT 61

COM=IY-FACE MOOERATOR YOID'

CUBOID O 1 2P7.665 2P1.2200 2P7.665

UNIT 62

COM=IX-FACE MOOERATOR VOID'

CUBOID O 1 2P1.2200 4P7.665

END GEOMETRY

READ ARPAY

$A R A=1$ MUX $=3$ MUY $=7 \quad M U Z=5$

$A R A=1$ NUX $=3$ NUY $=7 N U Z=5$

COAA $=$ NORTH SPLIT TABLE CORE

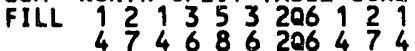

1042

1042,695356216153106566256 END FILL

$A R A=2$ NUX $=3$ NUY $=7$ NUZ $=5$

COM=ISOUTH SPLIT TABLE CORE

FILL 121353206121

1042

12563561206566256 END FILL

$A R A=3$ MUX $=1$ NUY $=1$ MUZ $=3$

COM=INORTH BOTTOM REFLECTOR'

FILL 141113 END FILL

ARA $=4 \quad N U X=1$ NUY=1 NUZ $=7$

COM=' SOUTH BOTTOM REFLECTOR'

II! 18 16 17 16 $16 \quad 1797$ END FILL

$A R A=5 \quad$ NUX $=1$ NUY $=1$ NUZ $=1$

COM ='EAST AND WEST WALLS OF NORTH REFLECTOR'

FILL $2 C$ END FILL 


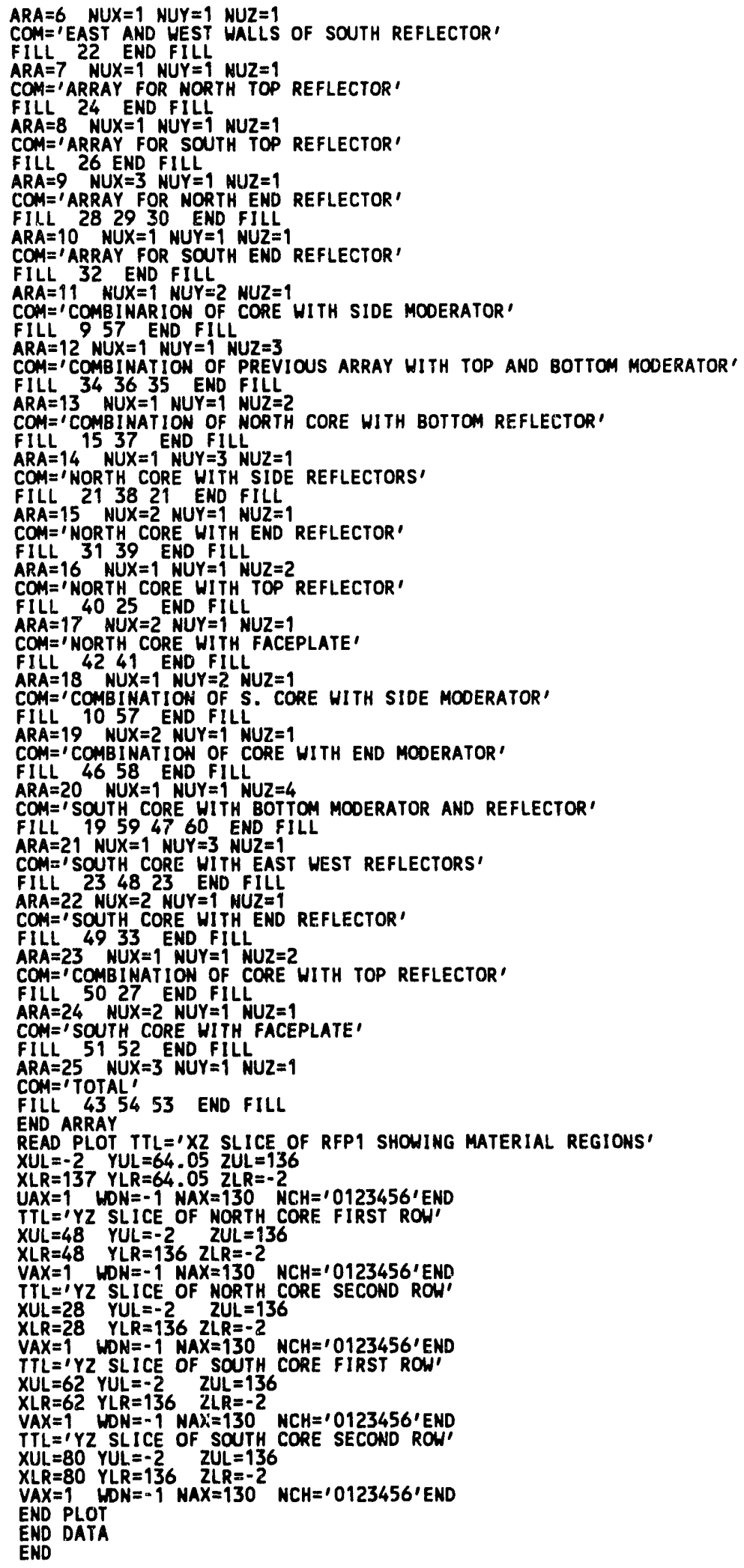




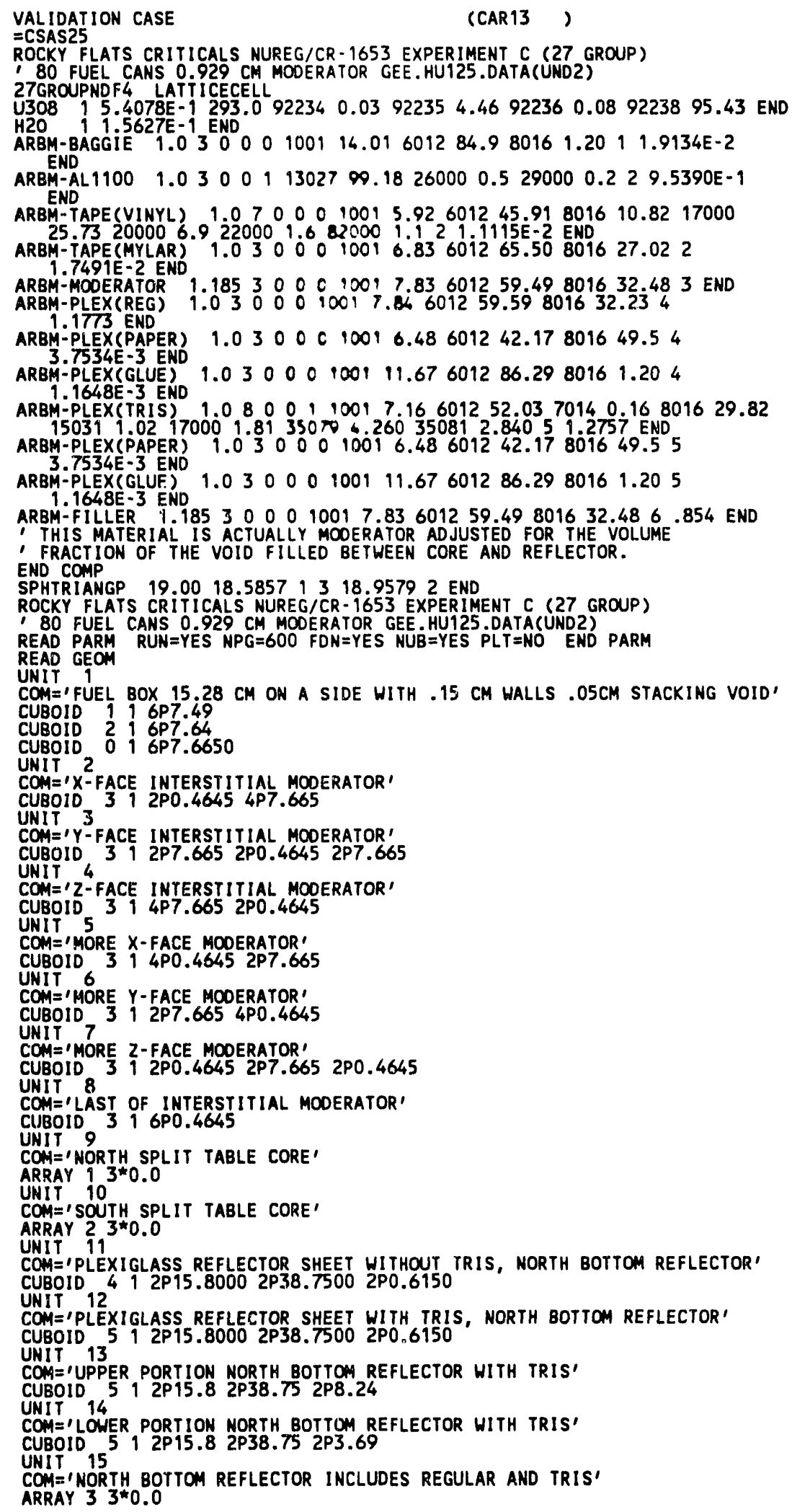


UNIT 16

COM='PLEXIGLAS SHEET BOTTOM SOUTH REFLECTOR WITHOUT TRIS'

CUBOID 012 P .12 P2.55 2 PO.615

CUBOID 4 1 $44.3-5.12 P 38.75$ 2PO.615

UNIT 17

COM='PLEXIGLAS SHEET BOTTOM SOUTH REFLECTOR WITH TRIS'

CUBOID 012 P $112 P 2.552 P 0.615$

CUBOID 5144.3 - 5.1 2P38.75 2P0.6150

UNIT 18

COM ='LOWER PORTION SOUTH BOTTOM REFLECTOR WITH TRIS'

CUBOID 012 P 1 . 1 2P2.55 2P8.855

CUBOID 5 1 44.3 - $5.12 P 38.75$ 2P8.855

COM=' SOUTH BOTTOM REFLECTOR WITH REGULAR AND TRIS'

ARRAY $43 * 0.0$

UNIT 20

TEST REFLECTORS FOR NORTH REFLECTOR HITH TRIS'

CUBOID 512 P15.8 2P12.65 2P54.2825

UNIT 21

COMA ='ARRAY FOR EAST AND WEST REFLECTORS FOR NORTH REFLECTOR'

ARRAY 53 3०0.0

UNIT 22

COM $=1 E A S T$ AND HEST REFLECTORS FOR SOUTH REFLECTOR WITH TRIS' CUBOID 512 2P24.700 2P12.65 2P54.2825

UNIT 23

COM $=$ AARAY FOR EAST AND HEST REFLECTORS FOR SOUTH REFLECTOR'

ARRAY $63 * 0.0$

UNIT 24

COH=IMORTH TOP REFLECTOR HITH TRIS

CUBOID 5 1 2 P28.35 2P64.05 2P12.15

UNIT 25

COM=1ARRAY FOR NORTH TOP REFLECTOR'

ARRAY $73 * 0.0$

UNIT 26

COM $=1$ SOUTH TOP REFLECTOR HITH TRIS

CUBOID 012 P 12 . 2 2.55 2P12.15

CUBOID 5169.4 -5.1 2P64.05 2P12.15

COM=1 RRRAY FOR SOUTH TOP REFLECTOR

ARRAY $83 * 0.0$

UNIT 28

CON $=1$ NORTH END REFLECTOR $9.8 \mathrm{CM}$ PORTION WITHOUT TRIS'

CUBOID 4 T 2P4.9000 2P64.0500 2P54.2825

UHIT 29

COM='NORTH END RELFECTOR $5.2 \mathrm{CM}$ PORTION WITH TRIS'

CUBOID 5 1 2 P2.6 2 P64.0500 2P54.2825

UNIT 30

COM ='NORTH END RELFECTOR $10.1 \mathrm{CM}$ PORTION WITHOUT TRIS

CUBOID 412 P5.05 2P64.05 2P54.2825

UNIT 31

COM = IARRAY FOR NORTH END REFLECTOR'

ARRAY $93 * 0.0$

ANIT 32

UNIT 32 TH END REFLECTOR,

CUBOID 5 , 2 P12.55 2P64.05 2P54.2825

UNIT 33

COM $=$ 'ARRAY FOR SOUTH END REFLECTOR'

ARRAY $103 * 0.0$

UNIT 34

COM $=112.95$ THICK MODERATING PLASTIC NORTH CORE

CUBOID 612 P 15.7945 2P6.4750 2P32.0535

UNIT 35

COM $=12.95$ THICK MODERATING PLASTIC NORTH CORE'

CUBOID 6 I 2P15.7945 2P38.5285 2P1.475

UNIT 36

3*0.0

UNIT 37

ARRAY $1233^{*} 0.0$

REPLICATE $010.0 \quad 0.011 \quad 0.443 \quad 0.0 \quad 0.1590 \quad 0.01$

UNIT 38

COM='NORTH CORE WITH BOTTOM REFLECTOR'

ARRAY 13 3* 0.0

UNIT 39

COM $=$ INORTH CORE WITH EAST AND WEST REFLECTOR

ARRAY $14 \quad 3 * 0.0$

UNIT 40 COM = 'NORTH CORE WITH ENO REFLECTOR'

ARRAY $153 \star 0.0$

UNIT 49

COM = INORTH SPLIT TABLE FACEPLATE

CUBOID 312 P0.6150 2P64.0500 2P66.4325

UNIT 42

COM $=$ 'NORTH CORE WIYH TOP REFLECTOR'

ARRAY $163 * 0.0$

UNIT 63

COM=INORTH CORE HITH FACEPLATE'

ARRAY $173 * 0.0$ 
UNIT 444 COM $=12^{4} .95$ CM THICK MODERATOR SOUTH CORE'

COM $=112.95$ CM THICK MODERATOR SOUTH CORE
CUBOID 6,2 P23.924 2 P6.475 2 P32.0535

UNIT $45^{\circ}$

COM $=12.95$ THICK MOOERATOR SOUTH CORE'

CUBOID 612 P23.924 2P38.5285 2 P1.475

UNIT 46

COM=I COMBINATION OF CORE WITH 12.95 THICK MODERATOR ARRAY $1833^{\star 0} 0.0$

UNIT 47

COM $=$ ' COMBINATION OF CORE WITH 2.95 THICK MODERATOR'

ARRAY $193 * 0.0$

REPLICATE $019 \begin{array}{lllllll}1.552 & 0.0 & 0.4430 & 0.0 & 0.159 & 0.0 & 1\end{array}$

UNIT 48 COM='SOUTH CORE WITH EAST WEST REFLECTORS'

ARRAY $203 \star 0.0$

UNIT 49

COM=' SOUTH CORE WITH EAST WEST REFLECTORS'

ARRAY $213 * 0.0$

UNIT 50

COM $=\prime$ SOUTH CORE END REFLECTOR

ARRAY $223 * 0.0$

UNIT 5

COM=I FACEPLATE FOR SOUTH SPLIT TABLE'

CUBOID 312 PO.4620 2P64.0500 2P66.4325

UNIT 52

COM $=$ ' SOUTH CORE HITH TOP REFLECTOR'

ARRAY $233 * 0.0$

UNIT 53

CORE HITH FACEPLATE

ARRAY $243 \star 0.0$

UNIT 54

COM=IAIR GAP

CUBOID 012 P $0.78052 P 64.05002$ P 66.4325

GLOBAL

UNIT 55

COM= 'TOTAL'

ARRAY $253 * 0.0$

UNIT 56

COM = INORTH BOTTOM MOOERATOR'

CUBOID 612 2P15.8000 2P38.7500 2P8.1295

UNIT 57

COM='BOTTOM SOUTH MODERATOR

CUBOID 692 P24.700 2P38.75 2P8.1295

UNIT 58

COM $=$ ' SOUTH REAR MOOERATOR'

CUBOID 31

END GEOMETRY

READ ARRAY

$A R A=1$ NUX $=3$ NUY $=7 \quad N U Z=7$

COM ='NORTH SPLIT TABLE CORE'

FILL 121353206121

4774688
2042

ARA $=2$ 2 $1353 \quad 206121$ END FILL

$\begin{aligned} & A R A=2 \\ & C O M\end{aligned}=1$ SOUTH SPLII TABLE CORE

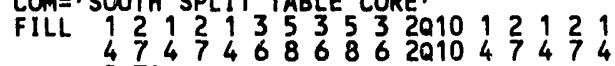
2070

1212135353201012121 END FILL

$A R A=3$ NUX $=1$ NUY $=1$ NUZ $=4$

COM='NORTH BOTTOM MCOERATOR AND REFLECTOR'

FILL $14 \quad 111356$ END FILL

$A R A=4 \quad N U X=1$ NUY $=1$ NUZ $=8$

COM $=$ ' SOUTH BOTTOM MODERATOR AND REFLECTOR'

FILL $18 \quad 16 \quad 17 \quad 16 \quad 16 \quad 971757$ END FILL

$A R A=5$ NUX $=1$ NUY $=1$ NUZ $=1$

ARA $=5$ NUX $=1$ NUY $=1$ NUZ $=1$
COM $=$ IEAST AND WEST WALIS OF NORTH REFLECTOR'

COM='EAST AND WEST

FILL 20 END FILL WUX NUY NUZ $=1$

COM='EAST AND WEST WALLS OF SOUTH REFLECTOR'

FILL 22 END FILL

$A R A=7 \quad N U X=1$ MUY $=1 \quad N U Z=1$

COM $=$ I ARRAY FOR NORTH TOP REFLECTOR'

FILL 24 END FILL

$A R A=8 \quad N U X=1$ NUY $=1 \quad N U Z=1$

COM ='ARRAY FOR SOUTH TOP REFLECTOR'

FILL 26 END FILL

$A R A=9$ NUX $=3$ NUY $=1$ NUZ $=1$

COM $=$ 'ARRAY FOR NORTH END REFLECTOR'

FILL 282930 END FILL

$A R A=10$ NUX $=1$ NUY $=1$ NUZ $=1$

COM=IARRAY FOR SOUTH END REFLECTOR'

FILL 32 END FILL

$A R A=11$ NUX $=1$ NUY $=2 \quad N U Z=1$

COM=' COMBINARION OF CORE WITH $12.95 \mathrm{CM}$ THICK MODERATOR' 
FILL 934 END FILL

$A R A=12$ NUX=1 NUY $=1$ MUZ $=2$

COM $=$ ' COMBINATION OF PREVIOUS ARRAY WITH $2.95 \mathrm{CM}$ THICK MODERATOR'

FILL 3635 END FILL

$A R A=13$ NUX $=1$ NUY $=1$ NUZ $=2$

COM= ' COMBINATION OF NORTH CORE WITH BOTTOM REFLECTOR'

FILL 1537 END FILL

$A R A=14$ NUX $=1$ NUY $=3$ NUZ $=1$

COM= 'NORTH CORE WITH SIDE REFLECTORS'

FILL 213821 END FILL

$A R A=15$ NUX $=2$ NUY $=1$ TUZ $=1$ REFECTOR'

COM= NORTH CORE WITH END

ARA $=16$ NUX 31 END FILL

ARA $=16$ NUX $=1$ NUY $=1$ NUZ $=2$
COM $=$ I NORTH CORE WITH TOP REFLECTOR'

FILL 4025 END FILL

$A R A=17$ NUX $=2$ NUY $=1$ NUZ $=1$

COM='NORTH CORE HITH FACEPLATE

FILL 4241 END FILL

$A R A=18$ NUX $=1$ NUY $=2$ NUZ $=1$

COM=' COMBIMATION OF S. CORE WITH 12.95 CM THICK MOOERATOR'

FILL 10 ARA 44 END FILL

ARA $=19$ NUX $=1$ NUY $=1$ NUZ $=2$ WITH $2.95 \mathrm{CM}$ THICK MOOERATOR'

COM= COMB INATION OF COR

$A R A=20$ NUX $=1$ NUY $=1$ NUZ $=2$

COM='SOUTH CORE WITH BOTTOM REFLECTOR'

FILL 1947 END FILL

$A R A=21$ NUX $=1$ NUY $=3$ NUZ $=1$

COM $=$ 'SOUTH CORE WITH EAST WEST REFLECTORS'

FILL $23 \quad 4823$ END FILL

COM=' SOUTH CORE WITH END REFLECTOR'

FILL 4933 END FILL

$A R A=23$ MUX $=1$ NUY $=1$ NUZ $=2$

$A R A=23$ NUX $=1$ NUY $=1$ NUZ $=2$ WIT TOP REFLECTOR'

COM=' COMBINATION OF CORE

FILL 5027 NUX 27 EMD FILL

COM='SOUTH CORE HITH FACEPLATE'

FILL 5152 END FILL.

$A R A=25$ NUX $=3$ NUY $=1$ NUZ $=1$

COM=' TOTAL'

FILL 435453 END FILL

END ARRAY

READ PLOT TTL $=1 X Z$ SLICE OF RFP3 SHOWING MATERIAL REGIONS'

$X U L=-1$ YUL $=64.05 \quad 2 U L=135$

$X L R=136 \quad Y L R=64.05 \quad 2 L R=-2 \quad 12 H=10123456^{\prime}$ END

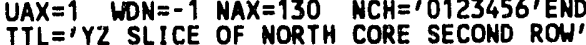

TTL $=Y Y Z$ SLICE OF NORTH CO

$\begin{array}{lll}X U L=28 & Y U L=-2 & 2 U L=136 \\ X L R=28 & Y L R=136\end{array}$

$X L R=28 \quad Y L R=136 \quad Z L R=-2$

VAX $=1$ WDN $=-1$ NAX $=130^{2}$ NCH $=10123456^{\prime}$ END

TTL $=$ 'YZ SLICE OF SOUTH CORE SECOND ROW'

$X U L=80$ YUL $=-2 \quad Z U L=136$

$X L R=80 \quad Y L R=136 \quad 2 L R=-2$

VAX $=1$ UDN $=-1$ MAX $=130^{\circ} \quad \mathrm{MCH}=10123456^{\circ}$

END PLOT

END DATA

END

VALIDATION CASE

(CAR15)

$=$ CSAS25

ROCKY FLATS CRITICALS NUREG/CR-1653 EXPERIMENT NUMBER ? (27 GROUP MOOEL) 1 FUEL CUBE $15.28 \mathrm{CM} U(4.46) 308$ a $H / U=1.25, H I$ CONC. SOLUTION DRIVEN

27GROUPNDF4 INFHOMMEDIUM

作

H2O 1 1.5627E-1 END $1.00114 .01601284 .980161 .2011 .9134 E-2$

ARBM-BACC

ARBM-AL1100 $1.030011302799 .18260000 .529000 \quad 0.22 \quad 9.5390 E-1$ END

ARBM-TAPE(VINYL) $1.077000010015 .926012 \quad 45.918016 \quad 10.82 \quad 17000$ $25.73200006 .9220001 .6820001 .121 .1115 \mathrm{E}-2$ END

ARBM-TAPE(MYLAR) $1.03000 \quad 10016.83601265 .508016 \quad 27.022$ 1.7491E-2 END

ARBM-MOOERATOR 1.185300010017 .83601259 .49801632 .483 END

ARBM-PLEX(REG) $\quad 1.0300010017 .84601259 .59801632 .234$

1.1773 END

ARBM-PLEX(PAPER) 1.0300010016 .48601242 .17801649 .54

3.75345E 3 END

ARBM-PLEX(GLUE) 1.03000100111 .67601286 .2980161 .204 $1.1648 E-3$ END

ARBM-PLEX(TRIS) $1.08800011001 \quad 7.16 \quad 6012 \quad 52.03 \quad 7014 \quad 0.16 \quad 801629.82$ 150311.02170001 .81350794 .260350812 .84051 .2757 END

ARBM-PLEX(PAPER) 1.0300010016 .48601242 .17801649 .55 3.7534E-3 END 
ARBM-PLEX(GLUE) 1.03000100111 .67601286 .2980161 .205 $1.1648 E-3$
END

ARBM-FILLER THIS MATERIAL IS ACTUALLY MOOERATOR ADJUSTED FOR THE VOLUME

? THIS MATERIAL IS ACTUALLY MOOERATR ADSUSTED FOR THE VOR

FRACTION OF THE VOID FILLED BETWEEN CORE AND REFLECTOR.
ARBM-AL 11001.0301091502799 .18260000 .5290000 .272 .715 END

SOLNUO2 (NO3)2 83351.180 .5491 .0293 .0922341 .0229223593 .172 922360.434922385 .372 END

5530491.0 END

END COMP

ROCKY FLATS CRITICALS NUREG/CR-1653 EXPERIMENT NUMBER ? (27 GROUP MODEL)

1 FUEL CUBE $15.28 \mathrm{CM} U(4,46) 308$ a $H / U=1.25$ HI COMC. SOLUTION DRIVEN

READ PARM NPG $=600$ NÚB $=Y E S$ SON $=Y E S$ PL $T=N 0^{\circ}$ END PARM

READ GEOM

COM=IFUEL BOX WITH VOID AND PART OF AL BOX'

CUBOID $019.80 \quad 0.03 .75 \quad 0.0 \quad 14.980 .0$

CUBOID $219.95 \quad 0.0 \quad 3.90 \quad-0.15 \quad 14.98 \quad 0.0$

CUBOID 1 i $14.98 \quad 0.0 \quad 9.365-5.615 \quad 14.980 .0$

UNIT 2

COM='FRONT OF FUEL BOX'

CUBOID $2,0.150 .0 \quad 5.6150 .014 .980 .0$

CUBOID 0 i $0.150 .0 \quad 9.3650 .014 .980 .0$

CUBOID $210.150 .014 .98 \quad 0.014 .980 .0$

UNIT 3

COM=IREAR OF FUEL BOXI

$\begin{array}{llllllll}\text { CUBOID } 2 & 1 & 0.15 & 0.0 & 14.98 & 0.0 & 14.98 & 0.0\end{array}$

CONIT 4 ARRAY TO ASSEMBLE PART OF BOX'

ARRAY $2633^{\star 0} 0$

UNIT 5

COM=I TOP AND BOTTOM OF BOX

CUBOID $019.800 .03 .750 \quad 0.0 \quad 0.150 .0$

CUBOID 2 i $15.28 \quad 0.0 \quad 9.365 \quad-5.615 \quad 0.15 \quad 0.0$

GLOBAL

UNIT 6

COM $=$ ARRAY TO ASSEMBLE TOP AND BOTTOM

ARRAY $273 * 0$

$\begin{array}{llllllll}\text { CUBOID } 2 & 1 & 15.28 & 0.0 & 15.13 & -0.15 & 15.28 & 0.0\end{array}$

CUBOID $0115.305-0.025 \quad 15.155-0.175 \quad 15.305-0.025$

UNIT 7

COM=I FUEL BOX $15.28 \mathrm{cM}$ ON A SIDE WITH $.15 \mathrm{cM}$ WALLS .05CM STACKING VOID'

CUBOID 196 P 7.49

CUBOID 216 GP7.49

CUBOID 0 i 6 P 7.6650

CUROID 34

COM=IALUMINUM WEIGHT DISTRIBUTION PLATE FOR SPECIAL BOX

CUBOID 019.800 .03 .7500 .00 .160 .0

CUBOID 7 T 7 15.33 $0.09 .54-5.790 .160 .0$

COM=IALUMINUM WEIGHT DISTRIBUTIOH PLATE FOR BOXES

CUBOID 79 4P7.665 0.160 .0

UNIT 9

COM=INORTH SPLIT TABLE CORE'

ARRAY $13 * 0.0$

CUBOID $0130.66 \quad-0.94 \quad 77.5 \quad 0.0 \quad 83.475 \quad 0.0$

COM=' SOUTH SPLIT TABLE CORE

ARRAY $233^{\circ} 0.0$

CUBOID $0199.4 \quad 0.0 \quad 77.5 \quad 0.0 \quad 83.475 \quad 0.0$

UNIT 11

COM='PLEXIGLASS REFLECTOR SHEET WITHOUT TRIS, NORTH BOTTOM REFLECTOR'

CUBO10 412 P 15.80002 P38.7500 2P0.6150

UNIT 12

COM='PLEXIGLASS REFLECTOR SHEET WITH TRIS, NORTH BOTTOM REFLECTOR'

CUBOID 512 P15.8000 2P38.7500 2P0.6150

UNIT 13

COM=IUPPER PORTION NORTH BOTTOM REFLECTOR WITH TRIS。

CUBOID 5 , 2 P 15.82 P 38.752 PB.24

UNIT 14 12 P15.8 2 P38.75 2 P8.24

COM $=$ 'LOWER PORTION NORTH BOTTOM REFLECTOR WITH TRIS'

CUBOID 512 P 15.82 P 38.752 P3.69

UNIT 15

COM='NORTH BOTTOM REFLECT'JR INCLUDES REGULAR AND TRIS'

ARRAY $33^{\star} 0.0$

UNIT 16

COM='PLEXIGLAS SHEET BOTTOM SOUTH REFLECTOR WITHOUT TRIS'

CUBOID 012 P5 12 P2.5 2 P0.6150

CUBOID $4144.3-5.12 P 38.75$ 2P0.615

UNIT 17

COM='PLEXIGLAS SHEET BOTTOM SOUTH REFLECTOR WITH TRIS'

CUBOID 012 P5.12P2.5 2 P0.6150

CUBOID 5 T $44.3^{1}-5.12 P 38.752 P 0.6150$

UNIT 18

COM ='LOWER PORTION SOUTH BOTTOM REFLECTOR WITH TRIS'

CUBOID 012 P 5.12 2P2.5 2 P8.855

CUBOID 5 T 44.3 -5.1 2 P38.75 2 P8.855 


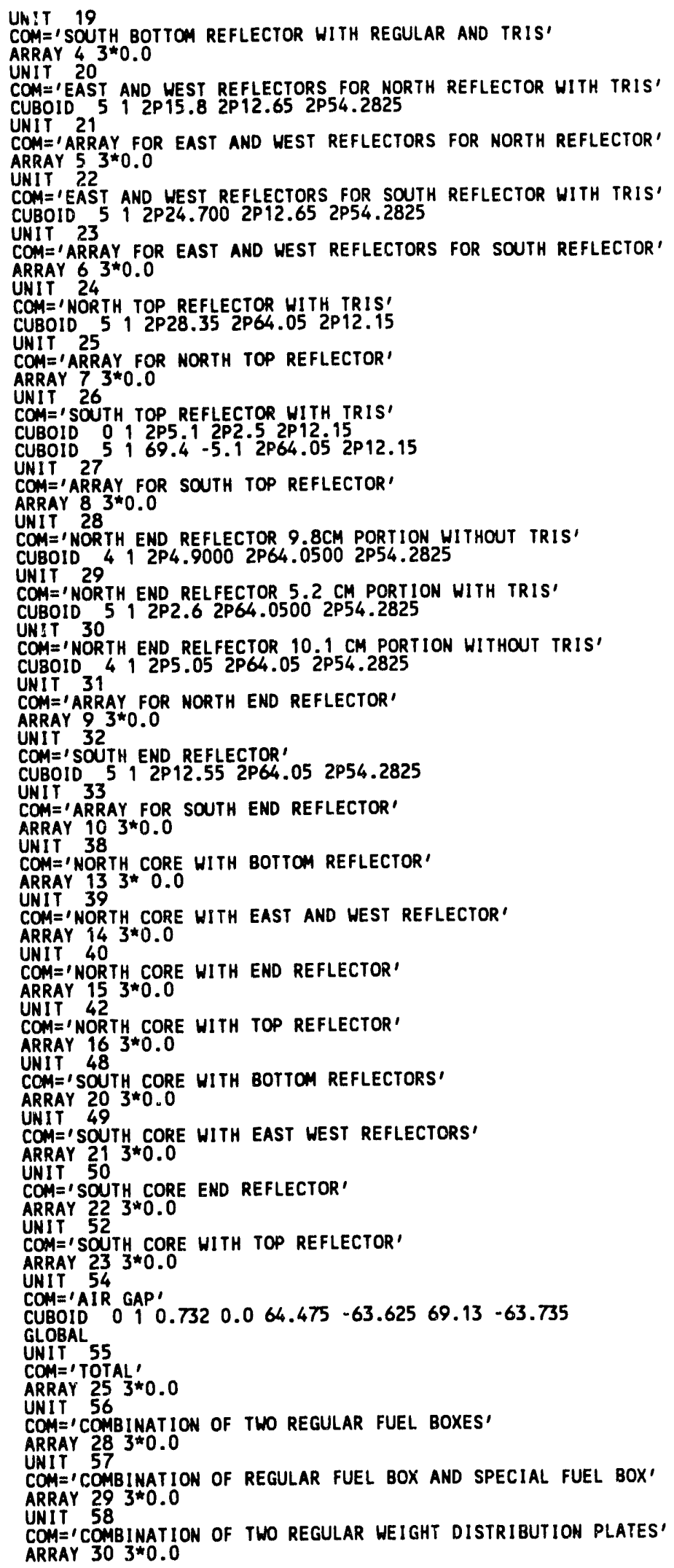




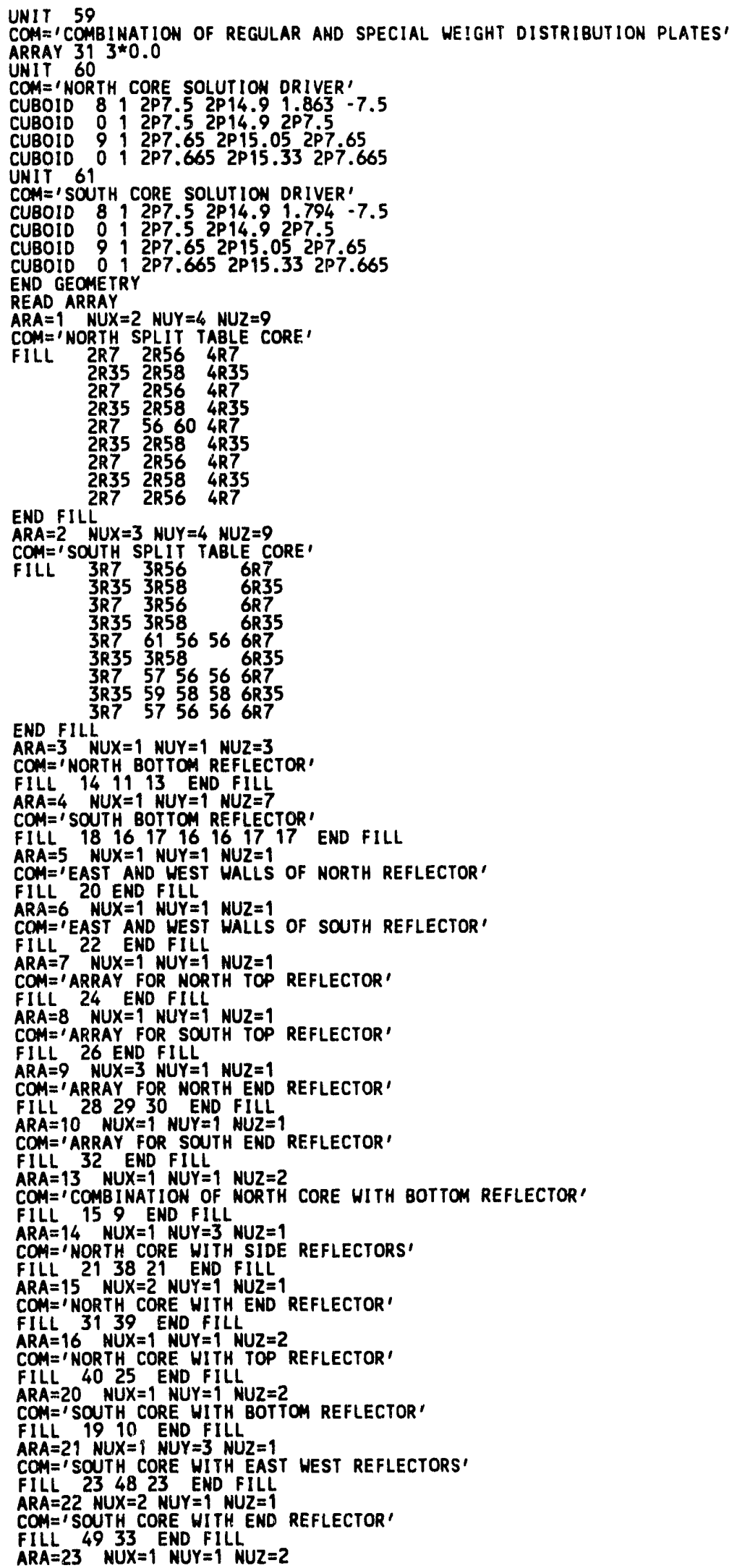




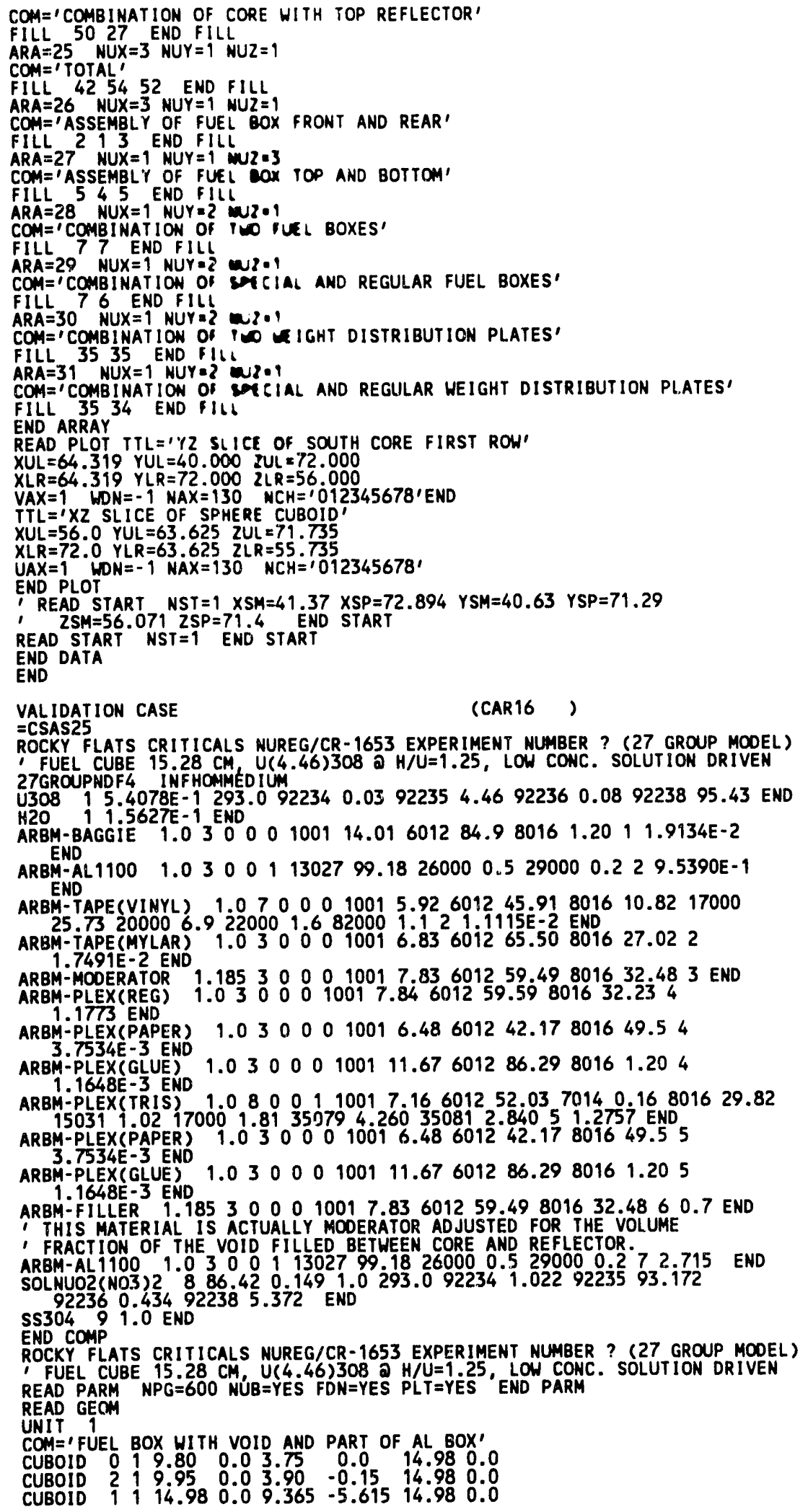


UNIT 2

COM $=1$ FRONT OF FUEL BOX'

COMBOID $2,0.150 .0 \quad 5.6150 .014 .980 .0$

CUBold $010.150 .0 \quad 5.6150 .014 .980 .0$

CUBOID $210.15 \quad 0.0 \quad 14.98 \quad 0.014 .980 .0$

UNIT 3

COM='REAR OF FUEL BOX

$\begin{array}{llllll} & & & \end{array}$

UNIT 4

COM=IARRAY TO ASSEMBLE PART OF BOX'

ARRAY 26 3*0

UNIT 5

COM $=1$ TOP AND BOTTOM OF BOX'

$\begin{array}{lllll} & & & \end{array}$

$\begin{array}{lllllllll}\text { CUBOID } & 0 & 9 & 9.80 & 0.0 & 3.750 & 0.0 & 0.15 & 0.0\end{array}$

GLOBAL

UNIT 6

COM='ARRAY TO ASSEMBLE TOP AND BOTTOM'

ARRAY $273 * 0$

$\begin{array}{llllllll}\text { CUBOID } 21 & 15.28 & 0.0 & 15.13 & -0.15 & 15.28 & 0.0\end{array}$

CUBOID $0115.305-0.025 \quad 15.155-0.175 \quad 15.305-0.025$

UNIT 7

COM='FUEL BOX $15.28 \mathrm{CM}$ ON A SIDE WITH .15 CM WALLS .05CM STACKING VOID'

CUBOID 11697.49

CUBOID 21 6P7.64

CUBOID 01 6P7.6650

UNIT 34

UNIT 34 MINUM YEIGHT DISTRIBUTION PLATE FOR SPECIAL BOX'

CUBOID 0
CUBOID $79.800 .03 .750-0.0 \quad 0.160 .0$

CUBOIT 35

COM=IALUMINUM WEIGHT DISTRIBUTION PLATE FOR BOXES'

CUBOID 7 1 4 P7.665 0.160 .0

UNIT 9

COM='NORTH SPLIT TABLE CORE'

ARRAY $13 * 0.0$

$\begin{array}{lllllll} & & \end{array}$

UNIT 10

COM='SOUTH SPLIT TABLE CORE'

ARRAY $23 * 0.0$

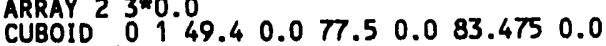

CUBOID 0149.40 .077 .50 .083 .4750 .0

UNIT 11 COMLXIGLASS REFLECTOR SHEET HITHOUT TRIS, NORTH BOTTOM REFLECTOR'

CUBOID 412 P 15.80002 2P38.7500 2P0.6150

UNIT 12 COM='PLEXIGLASS REFLECTOR SHEET WITH TRIS, NORTH BOTTOM REFLECTOR'

COM='PLEXIGLASS REFLECTOR SHEET HITH TRIS,
CUBOID 512 P 15.80002 P38.7500 2 PO.6150

UNIT 13

COM=IUPPER PORTION NORTH BOTTOM REFLECTOR HITH TRIS'

CUBCID 512 P15.8 2P38.75 2P8.24

UNIT 14 PORTION NORTH BOTTOM REFLECTOR HITH TRIS'

CUBOID 5 , 12 P 15.8 2P38.75 2 P3.69

UNIT 15

COMA NORTH
ARRAY $3 \quad 3 * 0.0$

UNIT 16 COM='PLEXIGLAS SHEET BOTTOM SOUTH REFLECTOR WITHOUT TRIS'

CUBOID 012 P5.1 2 P2.5 2 P0.6150

CUBOID 4 1 $44 . \dot{3}-5.12 P 38.752$ P0.615

UNIT 17

COM=IPLEXIGLAS SHEET BOTTOM SOUTH REFLECTOR WITH TRIS'

CUBOID $012 \mathrm{P} 512 \mathrm{P2} .52 \mathrm{P} 0.6150$

CUBOID 5 T $44.3-5.12 P 38.75$ 2P0.6150

UNIT 18 POM=ILOWER PORTION SOUTH BOTTOM REFLECTOR WITH TRIS'

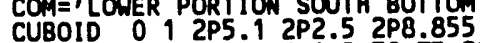

CUBOID $5144.3^{1}-5.12 P 38$. 55 2P8.855

UNIT , SOUTH BOTTOM REFLECTOR WITH REGULAR AND TRIS'

ARRAY $43 \star 0.0$

UNIT 20

COM =IEAST AND MEST REFLECTORS FOR NORTH REFLECTOR HITH TRIS'

CUBOID 512 P 15.82 P 12.652 P 54.2825

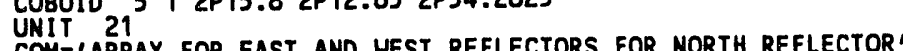

COM $=1$ ARRAY
ARRAY 5 \% $3 * 0.0$

ARRAY 52

COM='EAST AND WEST REFLECTORS FOR SOUTH REFLECTOR WITH TRIS'

CUBOID 512 P24.700 2P12.65 2P54.2825

UNIT 23 COM =IARRAY FOR EAST AND WEST REFLECTORS FOR SOUTH REFLECTOR'

ARRAY $63 * 0.0$

UNIT 24

UNIT 24.

CUBOID 5,12 P28.35 2 P64.05 2 P 12.15 
UNIT 25 COM= ARRAY FOR NORTH TOP REFLECTOR'

ARRAY $73 \star 0.0$

UNIT 26

COM=' SOUTH TOP REFLECTOR HITH TRIS'

CUBOID 0112 P5.1 2 P2.5 2 P 12.15

CUBOID 5169.4 -5.1 2P64.05 2P12.15

UNIT 27

COMI ='ARRAY FOR SOUTH TOP REFLECTOR'

ARRAY $83 \star 0.0$

UNIT 28

COM = NORTH END REFLECTOR $9.8 \mathrm{CM}$ PORTION WITHOUT TRIS'

CUBOID 4,2 P4.9000 2P64.0500 2P54.2825

UNIT 29

COM='NORTH END RELFECTOR $5,2 \mathrm{CM}$ PORTION HITH TRIS'

CUBOID 5 , 2 P2.6 2 P64.0500 2 P5 4.2825

UNIT 30

COM='NORTH END RELFECTOR $10.1 \mathrm{CM}$ PORTION WITHOUT TRIS'

CUBOID 412 P5.05 2P64.05 2P54.2825

UNIT 31

COM $=$ 'ARRAY FOR NORTH END REFLECYOR'

ARRAY $93 * 0.0$

UNIT 32

COM $=$ I SOUTH END REFLECTOR

CUBOID 512 2P12.55 2P64.05 2P54.2825

UNIT 33 YOY FOR SOUTH END REFLECTOR'

ARRAY 10 3*0.0

UNIT 38

COM='NORTH CORE WITH BOTTOM REFLECTOR'

ARRAY $133 * 0.0$

UNIT 39

COM $=$ 'NORTH CORE WITH EAST AND WEST REFLECTOR'

ARRAY $143 * 0.0$

UNIT 40

COM $=$ 'NORTH CORE WITH END REFLECTOR'

ARRAY $153 * 0.0$

UNIT 42

COM='NORTH CORE WITH TOP REFLECTOR'

ARRAY 16 3*0.0

UNIT 48

COM='SOUTH CORE WITH BOTTOM REFLECTORS'

ARRAY $203 * 0.0$

UNIT 49

COM='SOUTH CORE WITH EAST HEST REFLECTORS'

ARRAY $213 * 0.0$

COMIT $=$ SOUTH CORE END REFLECTOR'

ARRAY $223 * 0.0$

UNIT 52

COM=' SOUTH CORE WITH TOP REFLECTOR'

ARRAY 23 3*0.0

UNIT 54

COM='AIR GAPI

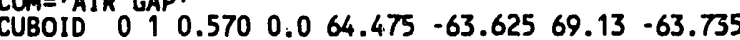

GLOBAL

UNIT 55

COM=1TOTAL,

ARRAY 25 3*0.0

UNIIT 56

CONIT 56 COMBINATION OF TWO REGULAR FUEL BOXES

ARRAY 283 3*0.0

UNIT 57

COMY =' COMBINATION OF REGULAR FUEL BOX AND SPECIAL FUEL BOX'

ARRAY $293 * 0.0$

UNIT 58

COM=' COMBINATION OF TWO REGULAR WEIGHT DISTRIBUTION PLATES'

ARRAY $303 * 0.0$

COMIT 59 ' COMBINATION OF. REGULAR AND SPECIAL WEIGHT DISTRIBUTION PLATES'

ARRAY $313 * 0.0$

ARRAY 60

UNIT 60

COM= $=$ NORTH CORE SOLUTION DRIVER

CUBOID 8 , 2 P $7.52 P 14.93 .332-7.5$

CUBOID $012 P 7.52 P 14.92 P 7.5$

CUBOID $912 P 7.652 P 15.052 P 7.65$

CUBOID 0 I 2 P7.665 2 P 15.332 PP7.665

UNIT 61 SOMTH CORE SOLUTION DRIVER'

CUBOID $812 P 7.52 P 14.93 .292-7.5$

CUBOID $812 P 7.5$ 2P 149237.5

CUBOID $912 P 7.652 P 15.052 P 7.65$

CUBOID $0192 \mathrm{P} 7.6652 \mathrm{P} 15.332 \mathrm{P} 7.665$

END GEOMETRY

READ ARRAY

$A R A=1 \quad$ NUX $=2$ NUY $=4 \quad$ NUZ $=9$

COM='NORTH SPLIT TABLE CORE' 


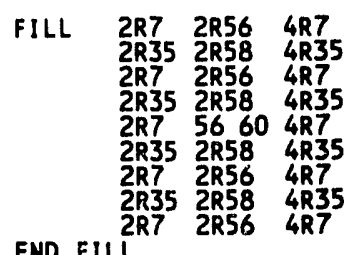

$A R A=2$ NUX $=3$ SUY $=4 \quad M U Z=9$ COM $=$ ISOUTH SPLIT TABLE CORE'

FIIL $3 R 73256$ ABLE 6 COE

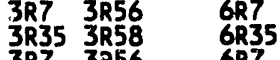

3273356067

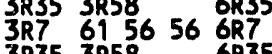

$3 R 7575656607$

$\begin{array}{lllll}3 R 7 & 57 & 56 & 56 & 6 R 7 \\ 3 R 35 & 59 & 58 & 58 & 6 R 35 \\ 3 R 7 & 57 & 56 & 56 & 6 R 7\end{array}$

END FIUL

$A R A=3$ MUX $=1$ NUY $=1$ NUZ $=3$

COM $=$ ' NORTH BOTTOM REFLECTOR

FILL 141113 END FILL

COM='SOUTH BOTTOM REFLECTOR'

FILL $1816 \quad 1716 \quad 1617 \quad 17$ END FILL

$A R A=5 \quad M U X=1 \quad$ NUY $=1$ MUZ $=1$

CORA $=$ IEAST AND WEST WALLS OF NORTH REFLECTOR'

COM= $E A S T$ N

FIL 20 END FILL $\quad$ KUZ

ARA $=6$ NUX $=1$ NUY $=1$ NUZ $=1$ OF SOUTH REFLECTOR'

COM='EAST AND MEST

$A R A=7$ NUX $=1$ NUY $=1$ NUZ $=1$

COM='ARRAY FOR MORTH TOP REFLECTOR'

FILL 24 END FILL

$A R A=8$ NUX $=1$ NUY $=1 \quad$ NUZ $=1$

COM=IARRAY FOR SOUTH TOP REFLECTOR'

FILL 26 END FILL

ARA =9 NUX $=3$ NUY $=1$ NUZ $=1$ REFLCTOR'

COM=ARRAY FOR NORTH END

FILL 282930 END FILL

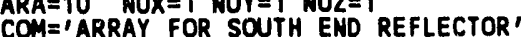

COM= ARRAY FOR SO

FIL! 32 END FILL

COA= 'COMBINATION OF HOKTH CORE WITH BOTTOM REFLECTOR'

FILL 159 END FILL

$A R A=14$ NUX $=1$ MUY $=3$ NUZ $=1$

COM='NORTH CORE HITH SIDE REFLECTORS'

FILL 2138 21 END FILL

ARA $=15$ NUX $X=2$ NUY $=1$ FUL REFLCTOR!

COM= NOR 3130 C

F

$A R A=16$ N $X=1$ TO

COMA'NORTH CORE WITH TOP

FILL 4025 END FILL

COH=' SOUTH CORE WITH BOTTOM REFLECTOR

FILL 1910 END FILL

$A R A=21$ NUX=1 NUY $=3$ NUZ $=1$

CON='SOUTH CORE WITH EAST WEST REFLECTORS'

FILL 234823 END FILL

ARA $=22$ NUX $=2$ NUY $=1$ NUZ $=1$ REFLECTOR'

FILL 4933 END FILL

$A R A=23$ NUX $=1$ NUY $=1$ NUZ $=2$

COM='COMBINATION OF CORE WITH TOP REFLECTOR'

FILL 5027 END FILL

$A R A=25$ NUX $=3$ NUY $=1$ NUZ $=1$

COM='TOTAL'

FILL 425452 END FILL

$A R A=26$ NUX $=3$ NUY $=1$ NUZ $=1$

COM=1ASSEMBLY OF FUEL BOX FRONT AND REAR

FILL 2 \& 3 END FILL 3 EN

CRA=27 NOX $=1$ NUY $=1$ NUZ=3

FILL 545 END FILL

ARA $=28$ NUX $=1$ NUY $=2$ NUZ $=1$

$C O M=$ COMBINATION OF THO FUEL BOXES

FILL 77 END FILL

$A R A=29$ NUX 1 MUY $=2$ NUZ $=1$

COM='COMBINATION OF SPECIAL AND REGULAR FUEL BOXES'

FILL 76 END FILL

$A R A=30$ MUX $=1$ NUY $=2$ NUZ $=1$

COM $=$ ' COMBINATION OF TWO WEIGHT DISTRIBUTION PLATES' 
FIL' 3535 END FILL

ARA $=31$ MUX $=1$ NUY $=2$ MUZ $=1$

COM=' COMBINATION OF SPECIAL AND REGULAR WEIGHT DISTRIBUTION PLATES'

FILL 3534 END FILL

END ARRAY

READ PLOT TTL='YZ SLICE OF SOUTH CORE FIRST ROW'

$X U L=64.319$ YUL $=40.000$ ZUL $=72.000$

$X L R=64.319$ YLR=72.000 ZLR=56.000

VAX $=1$ WDN $=-1$ MAXX $=130 \quad N C H=1012345678^{\prime}$ END

TIL $=1 X Z$ SLICE UF SPHERE CUBOID

$X U L=56.0$ YUL $=63.625 \quad 2 U L=71.735$

$X L R=72.0 \quad Y L R=63.625 \quad Z L R=55.735$

$\cup A X=1$ WDN $=-1$ NAX $=130$ NCH $=1012345678^{\prime}$

END PLOT

READ START NST $=1 \times S M=41.37$ XSP $=72.894 \quad Y S M=40.63 \quad Y S P=71.29$

ZSM $=56.071 \quad$ ZSP $=71.4 \quad$ END START

END DATA

END

VALIDAYION CASE (CAR18)

=CSAS25 25 COTS CRITICALS MUREG/CR-2500 EXPERIMENT G (27 GROUP)

130 FUEL CANS 2.44 CM MODERATOR GEE. HU2O3.DATA (OPTUM)

27GROUPNDF4 LATTICECELL

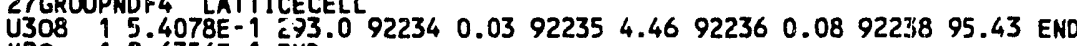

H2O $92.6356 \mathrm{E}-1$ END

ARBK-BAGGIE $1.03000100114 .01601284 .980161 .2011 .9134 E-2$

$\begin{array}{lllllllllllll}\text { END } & \\ \text { ARBM-ALL1100 } & 1.0 & 0 & 0 & 1 & 13027 & 99.18 & 26000 & 0.529000 & 0.2 & 2 & 9.5390 E-1\end{array}$ END

ARBM-TAPE(VINYL) $1.0700010015 .92601245 .918016 \quad 10.82 \quad 17000$

$25.73200006 .9220001 .6820001 .121 .1115 \dot{E}-2$ EMD

ARBM-TAPE (MYLAR) 1.0300010016 .83601265 .50801627 .022 1.7491E-2 END

ARBM-MOOERATOR 1.185300010017 .83601259 .49801632 .4 .83 END

ARBM-PLEX(REG) 1.0300010017 .84601259 .59801632 .234

$\begin{array}{llllllllll}1.1773 \text { END } & 10016.48601242 .17801649 .54\end{array}$

ARBM-PLEX(PAPER)

3.7534E-3 END 1.03000 300010.4

1.1648E-3 END

ARBM-PLEX(TRIS) $1.080010017 .16 \quad 601252.03 \quad 7014 \quad 0.16 \quad 8016 \quad 29.82$

$15031\} .02170001 .81350794 .260350812 .84051 .2757$ EIND

ARBM-PLEX(PAPER) 1.0300010016 .48601242 .17801649 .55 3.7 $354 \mathrm{E}-3 \mathrm{END}$

ARBM-PLEX (GLUE)

$1.0,000100111.67601286 .2980161 .205$

ARBM-FILLER 1.185300010017 .83601259 .49801632 .4861 .0 END

- THIS MATERIAL IS ACTUALLY MODERATOR ADJUSTED FOR THE VOLUME

- FRACTION OF THE VOID FILLED BETWEEN CORE AND REFLECTOR.

SPHTRIANGP 19.0018 .58571318 .95792 END

ROCKY FLATS CRITICALS NUREG/CR-2500 EXPERIMENT G (27 GROUP)

, 30 FUEL CANS 2.44 CM MOOERATOR GEE. HU203. DATA OPPTUM)

READ PARM RUN=YES NPG $=600$ FDN=YES NUB=YES PLT=NO

END PARM

READ GEOM

UNIT 1

COM='FUEL BOX $15.28 \mathrm{CM}$ ON A SIDE HITH .15 CM WALLS .05CM STACKING VOID'

CUBOID $116 \mathrm{P} 7.49$

CUBOID 29 6P7.64

CUBOID 2

COM=IX-FACF INTERSTITIAL MODERATOR

CUBOID 3 T 2P1.2200 4P7.665

UNIT 3

COM=YY $Y$ WACE INTERSTITIAL MODERATOR,

CUBOID 31 2P7.665 2P1.2200 2P7.665

CNIT 4

COM=1Z-FACE INTERSTITIAL MOOERATOR'

CUBOID 314 P 7.6652 P 1.2200

UNIT 5

COM $=$ MORE X-FACE MOOERATOR'

CUBOID 3 1 4P1.2200 2P7.665

UNIT 6

COM=IMOT.E Y-FACE MOOERATOR!

CÚBOIT 31 2P7.665 4P1.2200

UNIT

COM=IMORE Z-FACE MOOERATOR

CUBOID 3 1 2P1.2200 2P7.665 2P1.2200

UHit 8

COM $=\prime$ LAST OF IMTERSTITIAL MOOERATOR'

CUBOID 31 6P1.2200

UNIT 9

COM ='NORTH SPLIT TABLE CORE'

ARRAY $10^{+0.0}$ 
COM='SOUTH SPLIT TABLE CORE'

ARRAY 2 3*0.0

UNIT 11

COM='PLEXIGLASS REFLECTOR SHEET WITHOUT TRIS, NORTH BOTTOM REFLECTOR'

CUBOID 4 1 2 P 16.5500 2P38.7500 2P0.6150

UNIT 12

COM='PLEXIGLASS REFLECTOR SHEET WITH TRIS, NORTH BOTTOM REFLECTOR'

CUBOID $512 P 16.55002$ 2P38.7500 2P0.6150

UNIT 13

COM=IUPPER PORTION NORTH BOTTOM REFLECTOR HITH TRIS'

CUBOID 512 P 16.5500 2P38.75 2P8.24

UNIT 14 PORTION NORTH BOTTOM REFLECTOR HITH TRIS'

CUBOID 512 P 16.5500 2P38.73 2P3.69

UNIT 15 T 2 PI6.5SO 2 P38.TS 2 P3.69

COM=INORTH BOTTOM REFLECTOR INCLUDES REGULAR AND TRIS'

ARRAY $33 * 0.0$

UNIT 16 IO

CUBOID $012 \mathrm{PP} .12 \mathrm{P} 2.552 \mathrm{P} 0.615$

CUBOID 4 I 144.3 - $5.12 \mathrm{P} 38.752 \mathrm{PO} 0.615$

COM='PLEXIGLAS SHEET BOTTOM SOUTH REFLECTOR WITH TRIS'

CUBOID 0 I 2 P5.1 2 P2.55 2 P0.615

CUBOID $5144.3-5.12 P 38.752 P 0.6150$

CONIT 18 'LOWER PORTION SOUTH BOTTOM REFLECTOR WITH TRIS'

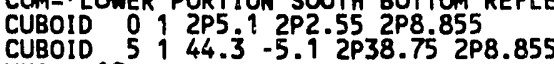

UNIT $19^{2}$

COM='SOUTH BOTTOM REFLECTOR WITH REGULAR AND TRIS'

ARRAY 4 3*0.0

UNIT 20 COM='EAST AND WEST REFLECTORS FOR NORTH REFLECTOR WITH TRIS'

CUBOID 512 1 16.5500 2P12.65 2P52.7825

UNIT 21

COM='ARRAY FOR EAST AND WEST REFLECTORS FOR NORTH REFLECTOR'

ARRAY $53 * 0.0$

UNIT 22

COM='EAST AND WEST REFLECTORS FOR SOUTH REFLECTOR WITH TRIS'

CUBOID 512 P24.700 2P12.65 2P52.7825

UNIT 23 TO

ARRAY $63 * 0.0$

UNIT 24

COM=INORTH TOP REFLECTOR WITH TRIS'

CUBOID 5 १ 2P29.1 2P64.05 2P12.15

UNIT 25

COM $=$ 'ARRAY FOR NORTH TOP REFLECTOR'

ARRAY $73 * 0.0$

UNIT 26

COM=I SOUTH TOP REFLECTOR WITH TRIS'

CUBOID 012 P 12 P2.55 2P12 15

CUBOID $5169.4-5.12 P 64.052 P 12.15$

UNIT 27 5 $69.4-5.12 P 64.052 P 12.15$

COM='ARRAY FOR SOUTH TOP REFLECTOR'

ARRAY $83 * 0.0$

UNIT 28

COM $=$ 'MORTH END REFLECTOR $9.8 \mathrm{CM}$ PORT ION WITHOUT TRIS'

CUBOID 412 P4.9000 2P64.0500 2P52.7825

UNIT 29

COM=' NORTH END RELFECTOR 5.2 CM PORTION WITH TRIS'

CUBOID 5 Y 5 2P2.6 2P64.0500 2P52.7825

COM=INORTH END RELFECTOR $10.1 \mathrm{CM}$ PORTION HITHOUT TRIS'

CUBOID 412 P5.05 2P64.05 2P52.7825

UNIT 31

COM='ARRAY FOR NORTH END REFLECTOR'

ARRAY $93 * 0.0$

UNIT 32

COM='SOUTH END REFLECTOR'

CUBOID 5 1 2P12.55 2P64.05 2P52.7825

UNIT 33

COM='ARRAY FOR SOUTH END REFLECTOR'

ARRAY 10 3*0.0

UNIT 34.95 THICK MODERATING PLASTIC NORTH CORE'

CUBOID 012 2P16.5500 2P13.315 2P25.4350

UNIT $=35$ THICK MOOERATING PLASTIC NORTH CORE'

CUBOID 612 P16.5500 2P38.75 2P1.7525

UNIT 36

ARRAY $113 * 0.0$

ARRAY $123 \star 0.0$ 


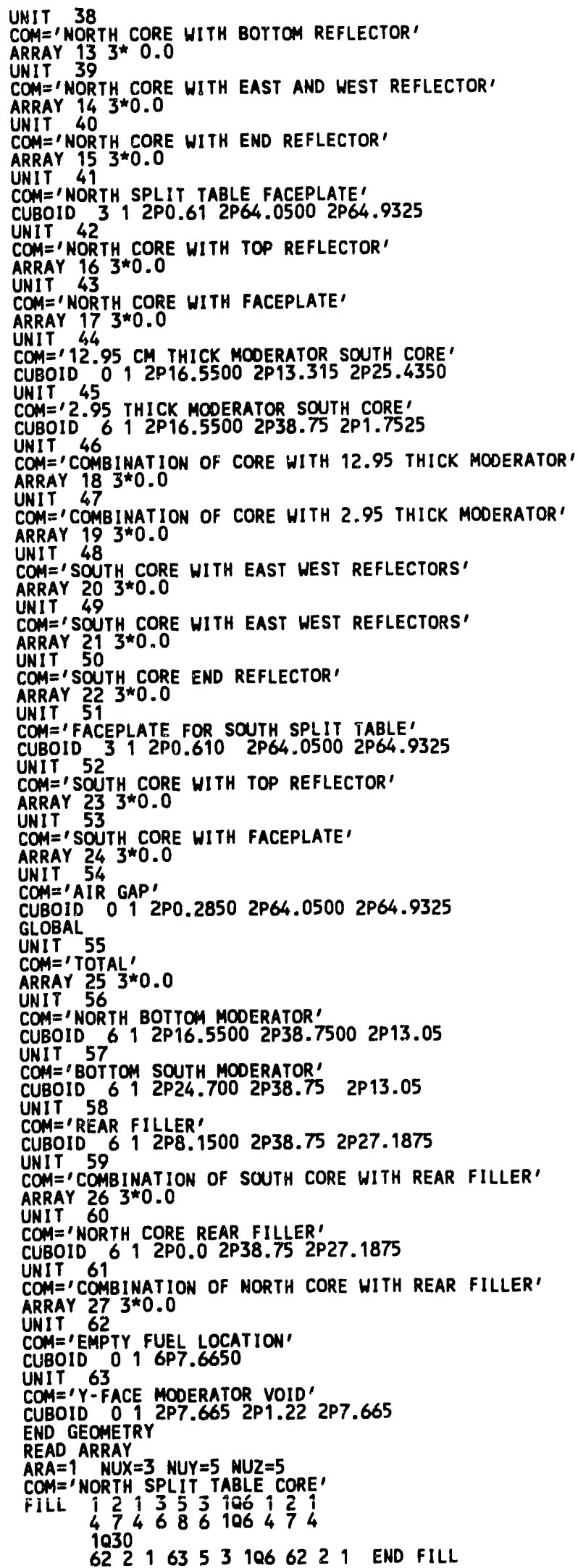


$A R A=2$ NUX $=3$ NUY $=5$ NUZ $=5$

COM='SOUTH SPLIT TABLE CORE

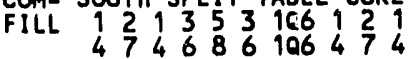
1030

126235631061262 END FILL

ARA $=3$ NUX $=1$ NUY $=1$ NUZ $=4$

COM='NORTH BOTTOM MODERATOR AND REFLECTOR'

FILL 14111356 END FILL

$A R A=4$ NUX $=1$ NUY $=1$ NUZ $=8$

COM ='SOUTH BOTTOM MODERATOR AND REFLECTOR'

FILL $18 \quad 16 \quad 17 \quad 16 \quad 1617 \quad 1757$ END FILL

$A R A=5 \quad M U X=1$ NUY $=1 \quad M U Z=1$

COM='EAST AND WEST WALLS OF NORTH REFLECTOR'

FILL 20 END FILL

$A R A=6$ NUX $=1$ NUY $=1 \quad$ MUZ $=1$

COM='EAST AND UEST WALIS OF SOUTH REFLECTOR'

FILL 22 END FILL

ARA $=7$ NUX=1 NUY=1 NUZ=1

COM='ARRAY FOR NORTH TOP REFLECTOR'

FILL 24 END FILL

$A R A=8$ NUX $=1$ NUY $=1 \quad$ NUZ $=1$

COM $=$ 'ARRAY FOR SOUTH TOP REFLECTOR'

FILL 26 END FILL

ARA $=9$ NUX $=3$ NUY $=1$ NUZ $=1$.

COM='ARRAY FOR NORTH END RI

FILL 282930 END FILL

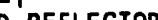

COM $=1$ ARRAY FOR SOUTH END REFLECTOR

FILL 32 END FILL

$A R A=11$ NUX $=1$ NUY $=2$ NUZ $=1$. $12.95 \mathrm{CM}$ THICK MODERATOR

FILL 934 END FILL

$A R A=12$ NUX $=1$ NUY $=1$ NUZ $=2$

COM $=$ 'COMBINATION OF PREVIOUS ARRAY WITH 2.95CM THICK MODERATOR'

FILL 36 35 END FILL

ARA $=13$ NOMU $=1$ NOY 1 OF NORTH CORE HITH BOTTOM REFLECTOR'

FILL 1569 END FILL

$A R A=14$ NUK $=1$ NUY $=3$ NUZ $=1$

COM='NORTH CORE WITH SIDE REFL.ECTORS

FILL 213821 END FILL

$A R A=15$ NUX $=2$ MUY $=1$ NUZ $=1$

COM=' NORTH CORE WITH END REFLECTOR'

FILL 3139 END FILL

$A R A=16$ MUX $=1$ NUY $=1$ NUZ $=2$

COM $=$ INORTH CORE WITH TOP REFLECTOR'

FILL 4025 END FILL

COM= MORTH COPE HITH FACEPLATE'

FILL 4241 END FILL

$A R A=18$ NUX $=1$ NUY $=2$ NUZ $=1$

COM=' COMBINATION OF S. CORE WITH 12.95 CM THICK MODERATOR'

FILL 1044 END FILL

$A R A=19$ NUX $=1$ NUY $=1$ NUZ $=2$

COM = ' COMBINATION OF CORE WITH 2.95 CM THICK MODERATOR'

FILL 4645 END FILL

$A R A=20$ C $A U X=1$ MUY $=1$ MUZ $=$ ?

CORA= I SOUTH CORE WITH BOTTOM REFLECTOR'

FILL 1959 END FILL

$F I L L 21959$, END $F I L L Z=1$

ARA $=21$ NUX $=1$ NUY $=3$ NUZ $=1$ WEST REFLECTORS'

COM= SOU 4823 END $F I L L$

FILL $23 \quad 4823$ END FILL

$A R A=22$ NUX $=2$ NUY $=1$ NUZ $=1$ REFECTOR

COM $=$ 'SOUTH CORE WITH END

FILL 4933 END FILL

COM $=$ ' COMBINATION OF CORE WITH TOP REFLECTOR'

FILL 5027 END FILL

FRA $=24$ NUX $=2$ NUYY $=1$ NUZ $=1$

$A R A=24$ NUX $=2$ NUY $=1$ NUZ $=1$

FILL 5152 END FILL

$A R A=25$ NUX $=3$ NUY $=1$ NUZ $=1$

COM $=$ I TOTAL

FILL 435453 END FILL

COM=' COMBINATION OF SOUTH CORE WITH REAR FILLER'

FILL 4758 END FILL

$A R A=27$ NUX $=2$ NUY $=1$ NUZ $=1$

ARA $=27$ NUXILATION OF NORTH CORE WITH REAR FILLER'

FILL 37 GO END FILL

FILL 37 ARRAY

READ PLOT TTL $=1 X Z$ SLICE OF RFPG SHOWING MATERIAL REGIONS'

$X \cup L=-1 \quad Y U L=64.05 \quad Z U L=135$

$X \perp P=136 \quad 11=64.05$ YIR $=-2$

UAX $=1$ WDH $=-1$ MAX $=130 \quad \mathrm{NCH}=10123456^{\prime} \mathrm{END}$

TTL $=$ 'YZ SLICE OF NORTH CORE FIRST RON 


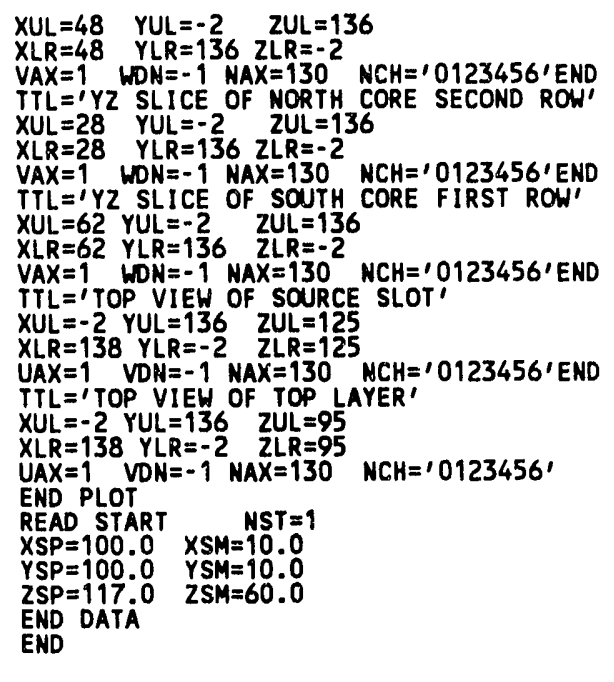

VALIDATION CASE (CAR2O)

=CSAS25 ROCKY FLATS CRITICALS NUREG/CR-2500 EXPERIMENT NUMBER ? (27 GROUP MOOEL) ROCKY FUEL CUBE $15.28 \mathrm{CM} U(4.46) 308$ Q $H / U=2.03$, HI ENRICHED SPHERE DRIVEN

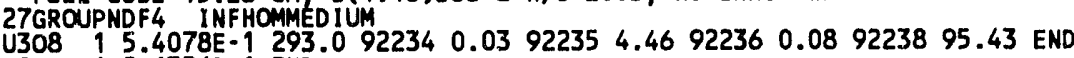
H2O $12.6356 \mathrm{E}-1$ EMD

ARBM-BAGGIE $1.030001001 \quad 14.01601284 .980161 .2011 .9134 E-2$ END

ARBM-AL1100 $1.0300011302799 .1826000 \quad 0.5290000 .229 .5390 E-1$

ARBM-TAPE(VINYL) $\quad 1.0700010015 .92601245 .918016 \quad 10.8217000$ $25.73200006 .9220001 .6820001 .121 .1115 \mathrm{E}-2$ END

ARBM-TAPE (MYLAR) 1.0300010016 .83601265 .50801627 .022 $1.7491 \mathrm{E}-2$ END

ARBM-MOOERATOR 1.1853000010017 .83601259 .49801632 .483 END

ARBM-PLEX(REG) 1.0300010017 .84601259 .59801632 .234 1.1773 END

ARBM-PLEX(PAPER) 1.0300010016 .48601242 .17801649 .54 3. $7534 \mathrm{E}-3$ END

ARBM-PLEX(GLUE) 1.03000100111 .67601286 .2980161 .204

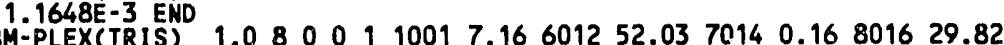

ARBM-PLEX (TRIS) $15031.0800350794 .260^{16} 350812.84051 .2757$ END

ARBM-PLEX(PAPER) 1.03 00010016.48601242 .17801649 .55 3.7534E-3 END

ARBM-PLEX(GLUE) 1.03000100111 .67601286 .2980161 .205 $1.1648 \mathrm{E}-3$ END

ARBM-FILLER 1.1853000010017 .83601259 .49801632 .4860 .7 END

I THIS MATERIAL IS ACTUALLY MOOERATOR ADJUSTED FOR THE VOLUME

- FRACTION OF THE VOID FILLED BETWEEN CORE AND REFLECTOR.

ARBM-AL $1100 \quad 1030011302799.182600005290000 .272715$ END

ARANIUM $0.9483293 .092234 \quad 190223593.19922360 .492238 \quad 5.41$ END

ARBM-PJ 0.81620000100185 .1601214 .980 .0089 END

SS304 9 9.0 END

SS304 COMP

ROCKY FLATS CRITICALS NUREG/CR-2500 EXPERIMENT NUMBER ? (27 GROUP MODEL) 1 FUEL CUBE $15.28 \mathrm{CM}$ U(4.46)308 a H/U =2.03 HI ENR ICHED SPHERE DRIVEN READ PARM NPG $=600$ NÚB=YES FDN=YES PLT $=$ NO END PARM

READ GEOM

UNIT 1

COM='FUEL BOX WITH VOID AND PART OF AL BOX'

CUBOID $019.800 .03 .75 \quad 0.0 \quad 14.980 .0$

CUBOID $219.950 .03 .90-0.1514 .980 .0$

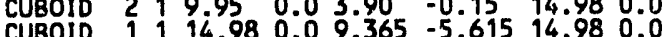

CUBOID

UNIT 2

COM=IFRONT OF FUEL BOX

CUBOID $2,0.150 .0 \quad 5.6150 .014 .980 .0$

CUBOID $010.150 .0 \quad 0.3650 .014 .980 .0$

CUBOID $210.150 .0 \quad 14.98 \quad 0.0 \quad 14.98 \quad 0.0$

UNIT 3

COM=IREAR OF FUEL BOX'

$\begin{array}{llllllll}\text { CUBOID } 2 & 1 & 0.15 & 0.0 & 14.98 & 0.0 & 14.98 & 0.0\end{array}$

UNIT 4 COM=IARRAY TO ASSEMBLE PART OF BOX'

ARRAY 26 3*0

COMITIOP AND BOTTON OF BOX'

$\begin{array}{lllllll} & & & \end{array}$

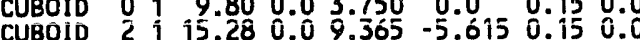




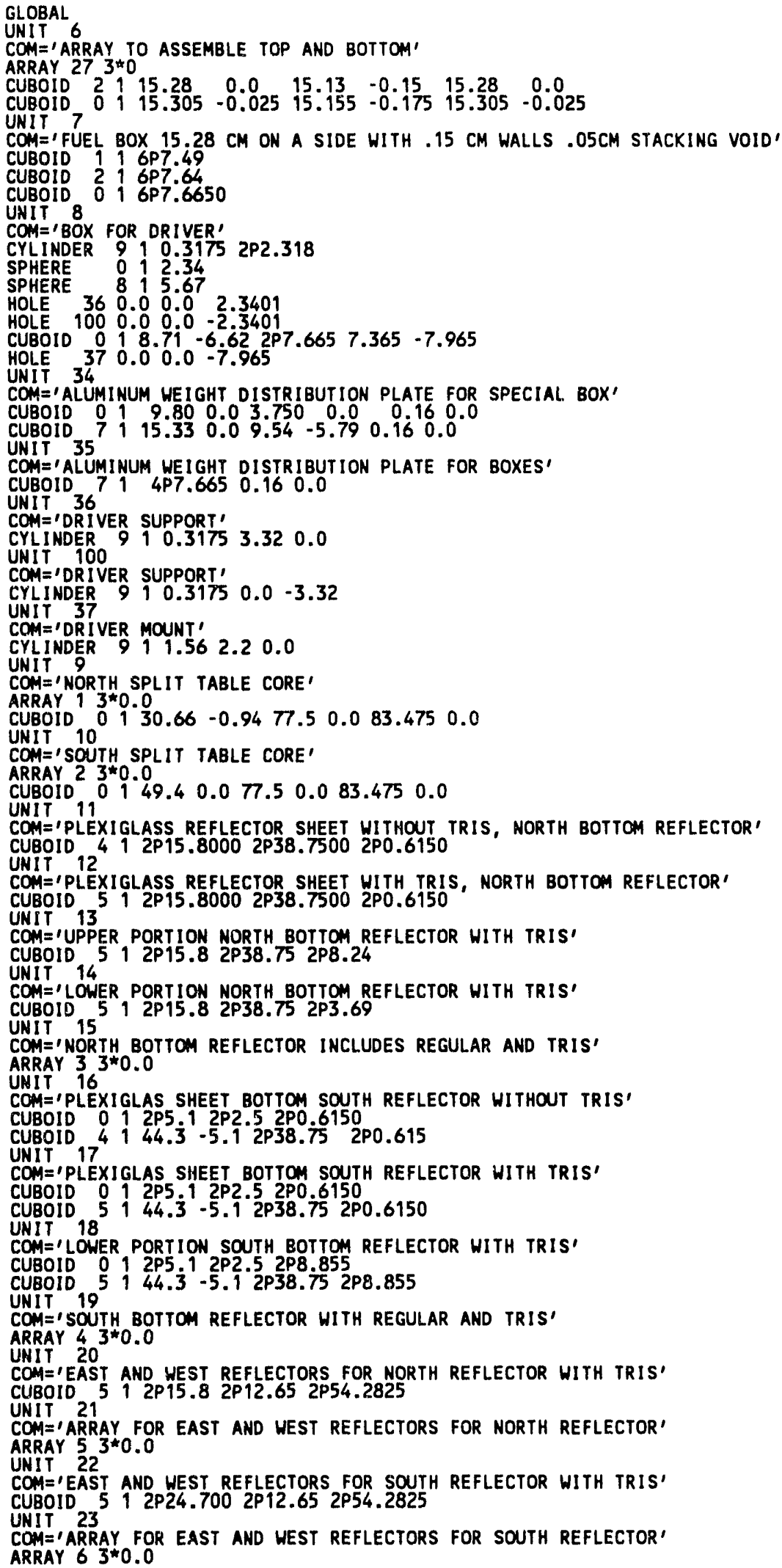




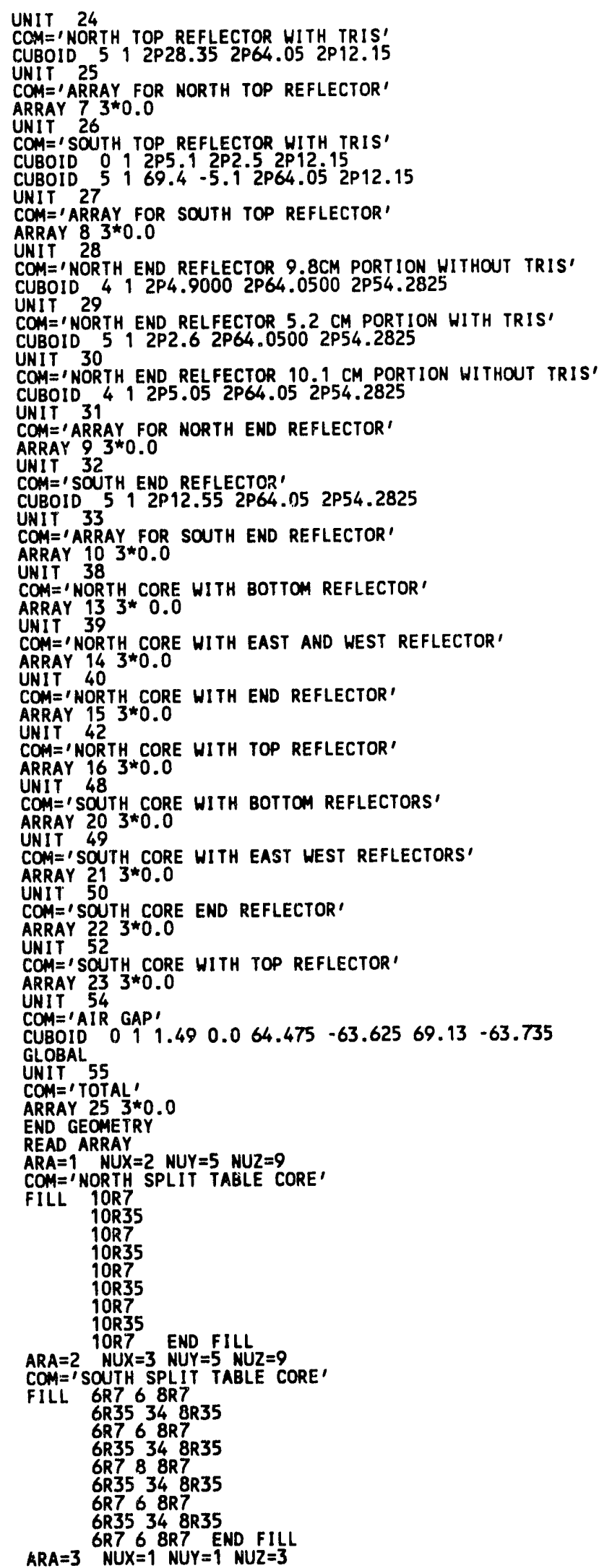




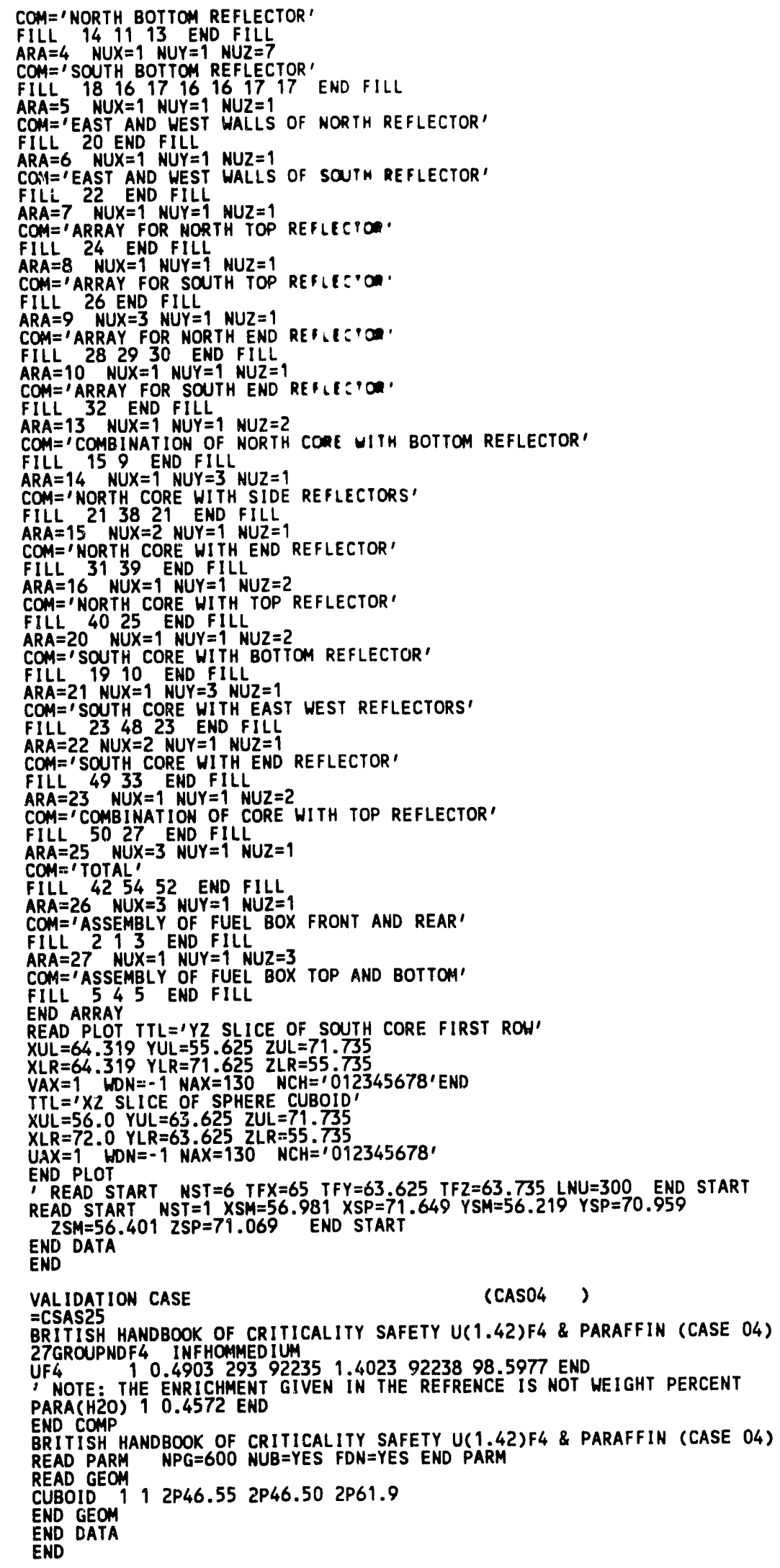




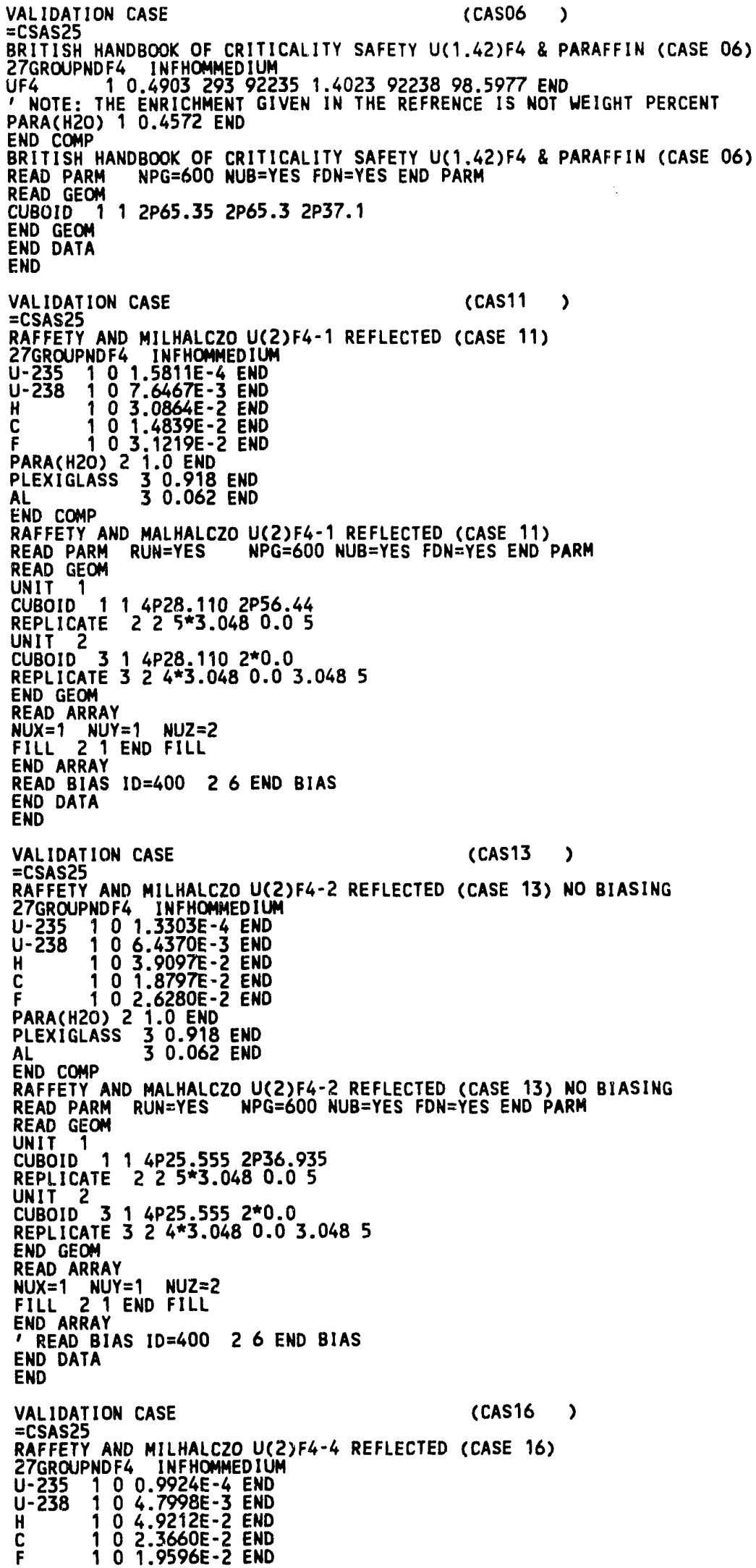




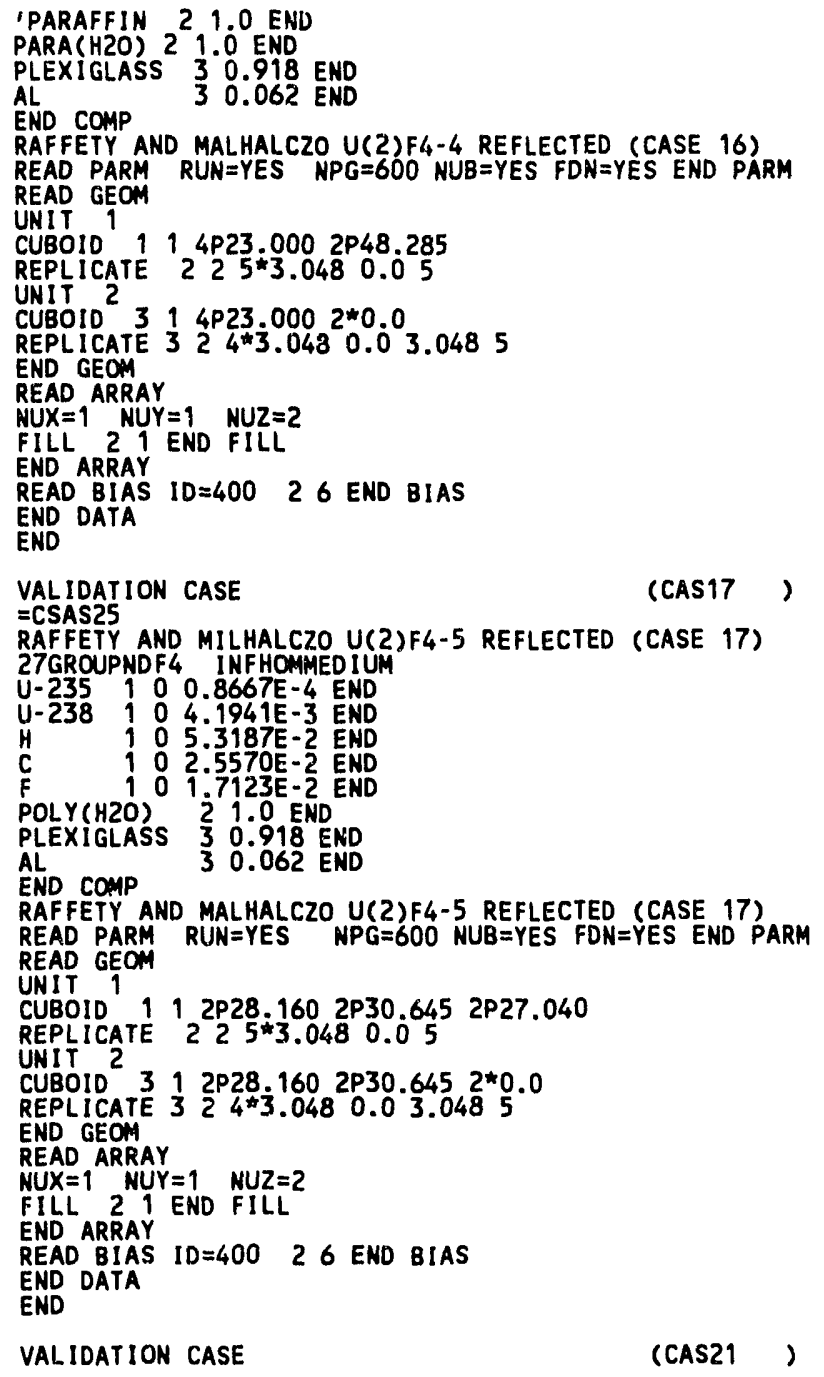

=CSAS25
RAFFETY AND MILHALCZO U(3)F4-1 REFLECTED (CASE 21) NO BIAS 27GROUPNDF 4 INFHOMMEDIUM $U-238$ i $07.4999 \mathrm{E}-3$ END H $103.1341 \mathrm{E}-2$ END

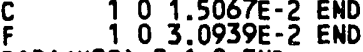

PARACH2O) 2 i.O END

PARA(H2O) 2 1.0 END END

AL 30.062 END

END COMP READ PARM RUN $=$ YES NPG $=600$ NUB $=$ YES FDN $=$ YES END PARM READ GEOM

UNIT 1

CUBO1D 112 P25.57 2P25.572P25.635

REPLICATE $225 * 3.0480 .05$

UNIT ${ }^{2} 3,2 P 25.572 P 25.572 * 0.0$

REPLICATE $324 \$ 3.0480 .03 .0485$

REPLICATE

END GEOM

NUX $=1$ ARUY $=1 \quad$ NUZ $=2$

FILL 2 I END FILL

END ARRAY

1 READ BIAS ID $=40026$ END BIAS

END DATA

END 


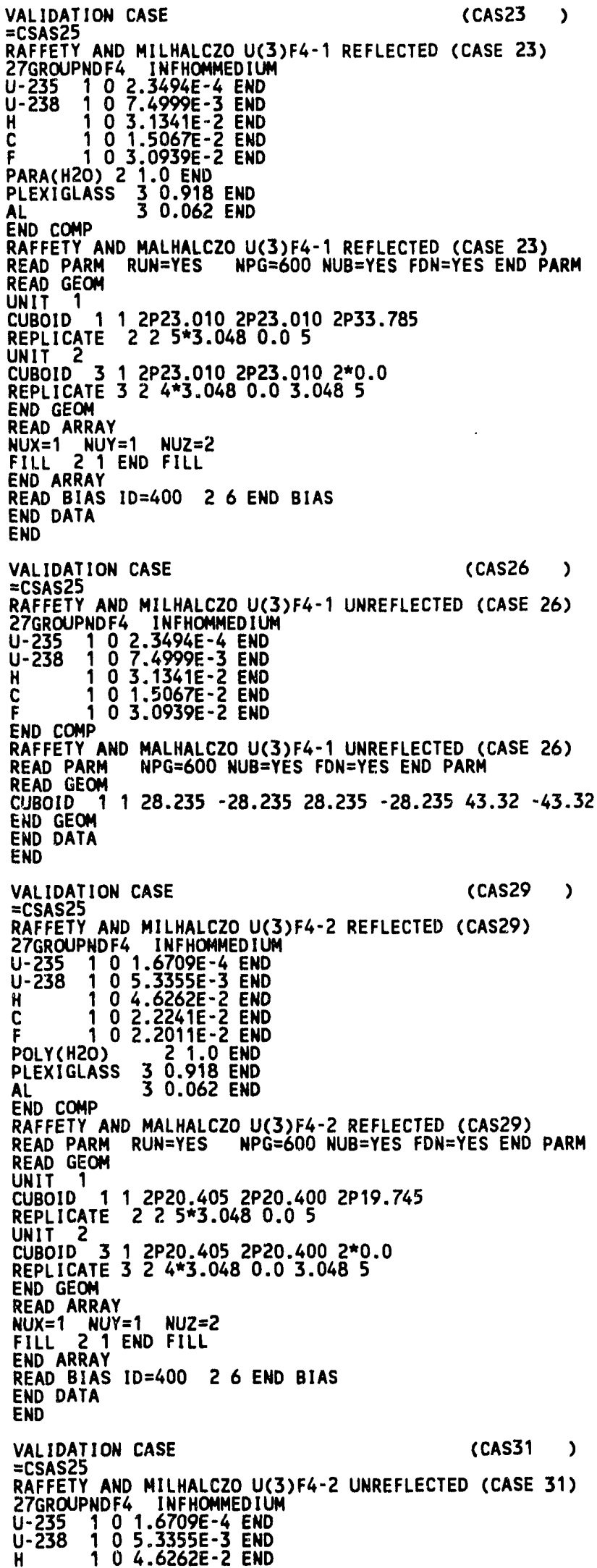




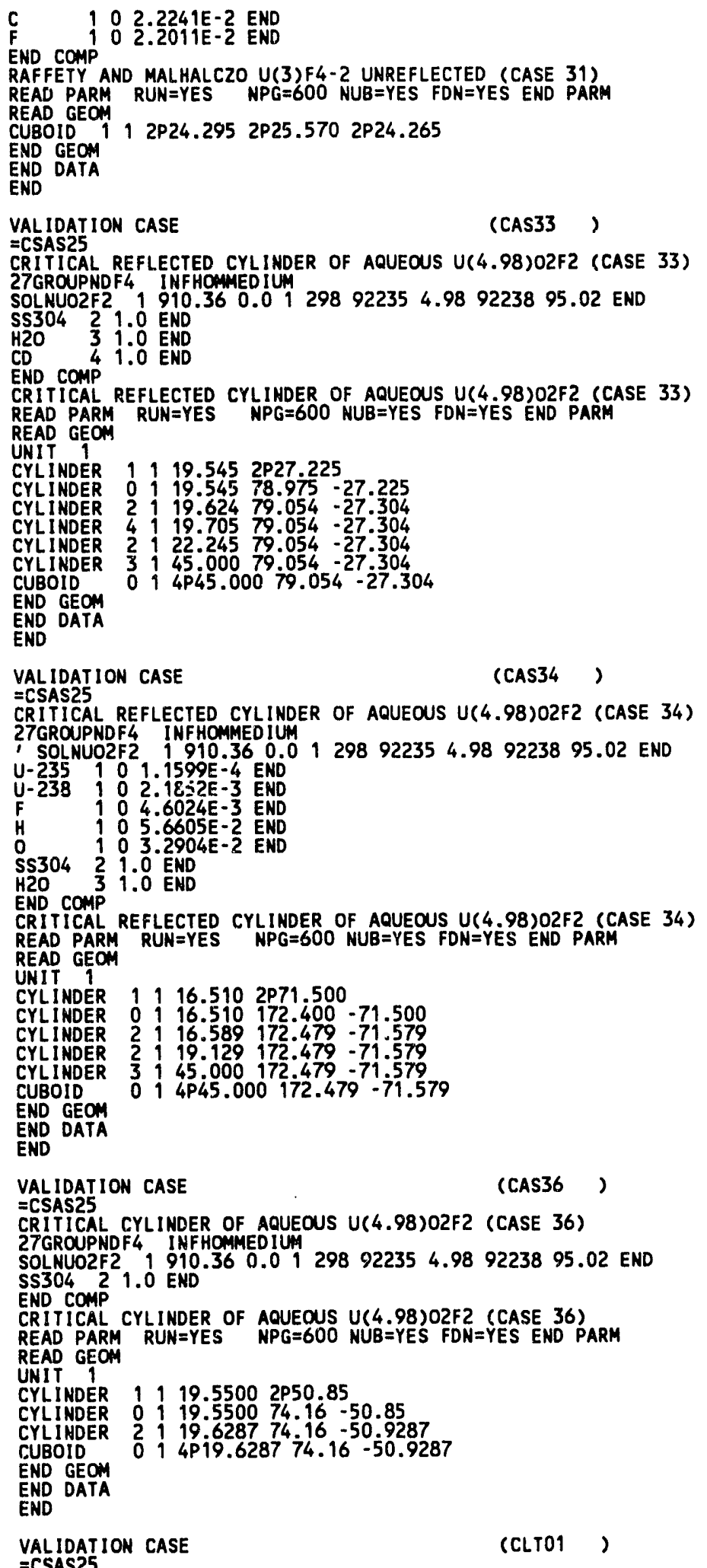

=CSAST

(CLT01)

BAW-1645-4, EXP'MT-2452, CASE C-2-C, DANCOFF FACTOR FOR EACH FUEL PIN 27GROUPNDF4 LATTICECELL

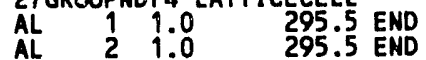




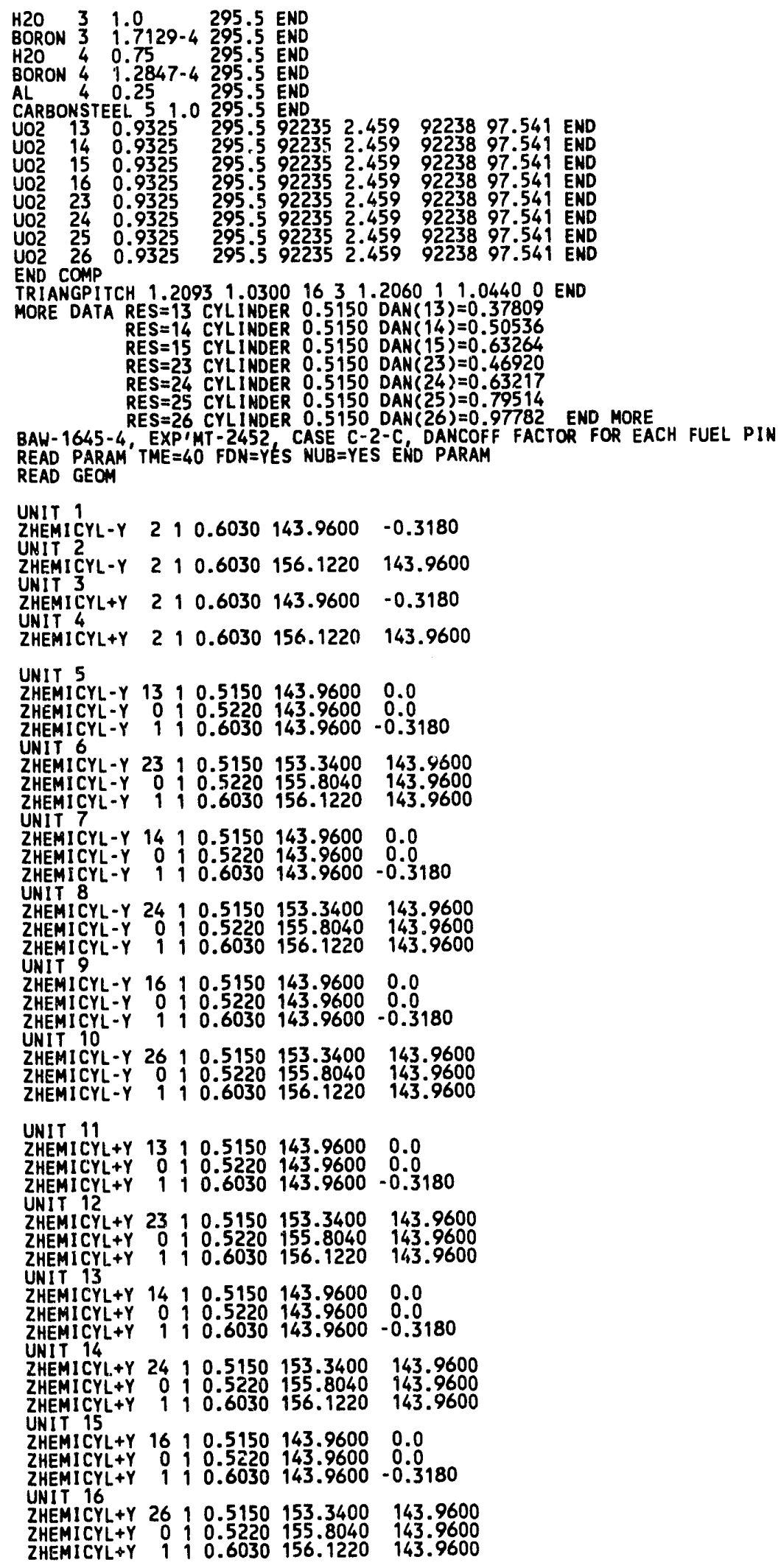




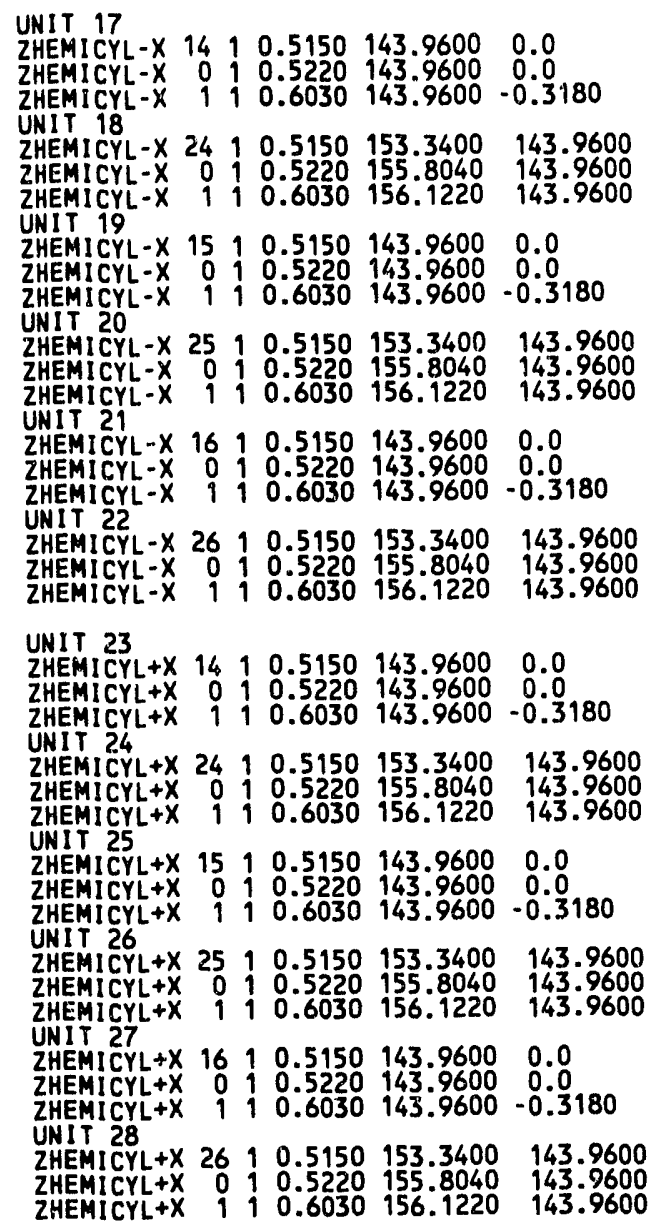

ZHEMICYL+X 190.6030156 .1220143 .9600

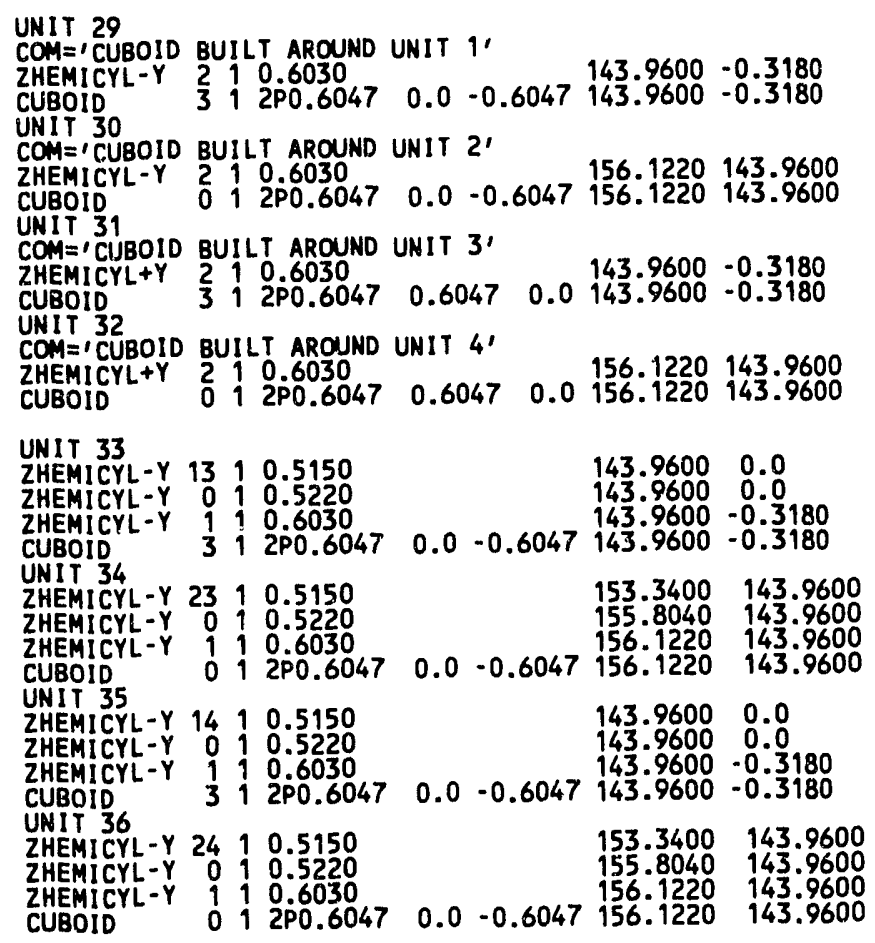




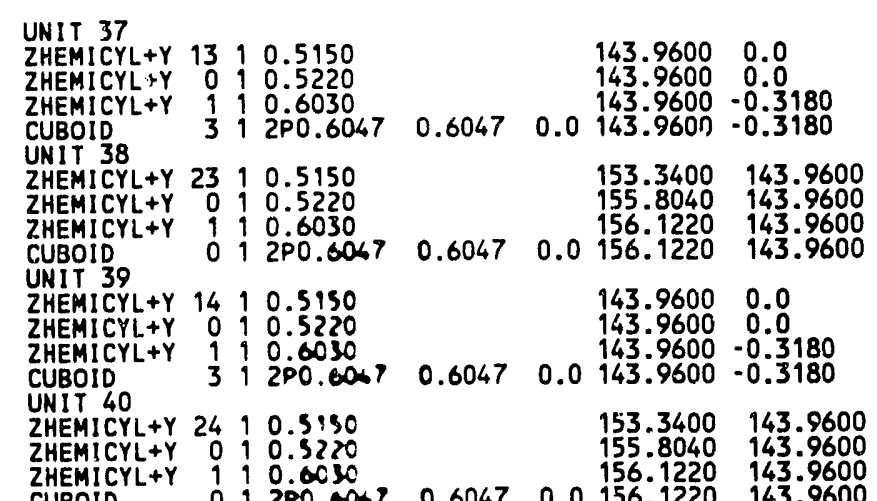

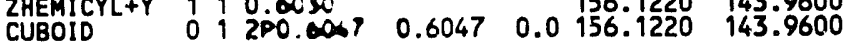

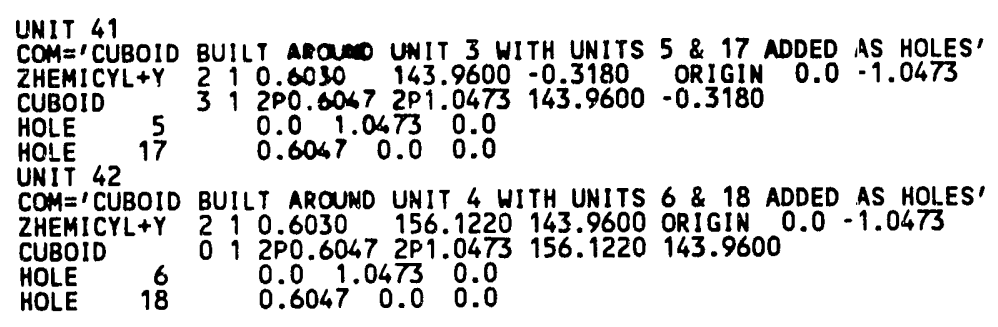

UNIT 43 COM='CUBOID BUILT AROUND UNIT 11 WITH UNITS $921 \& 23$ ADDED AS HOLES'

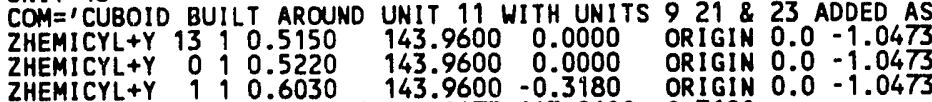

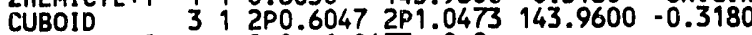

$\begin{array}{llllll}\text { HOLE } & 9 & 0.047 & 0473 & 0.0 \\ \text { HOLE } & 21 & 0.6047 & 0.0 & 0.0 \\ \text { HOLE } & 23 & -0.6047 & 0.0 & 0.0\end{array}$

COMIT 44 CUEOID BUILT AROUND UNIT 12 HITH UNITS $1022 \& 24$ ADDED AS HOLES

ZHEMICYL+Y $2310.5150 \quad 153.3400143 .9600$ ORIGIN $0.0-1.0473$

ZHEMICYL+Y $010.5220 \quad 155.8040143 .9600$ ORIGIN $0.0-1.0475$

ZHEMICYL+Y $1910.6030 \quad 156.1220143 .9600$ ORIGIN $0.0-1.0473$

CUBOID 012 PO0.6047 2P1.0473 156.1220 143.9600

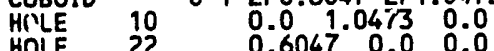

$\begin{array}{lllll}\text { HOLE } & 22 & 0.6047 & 0.0 & 0.0 \\ \text { HOLE } & 24 & -0.6047 & 0.0 & 0.0\end{array}$

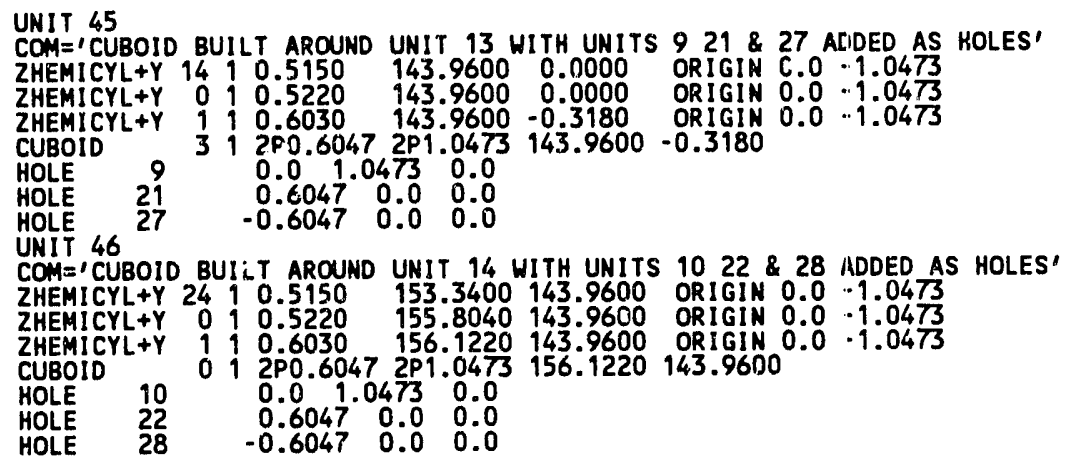

UNIT 47 COM=ICUBOID BUILT AROUND UNIT 11 WITH UNITS $917 \& 27$ ADDED AS HOLES' ZHEMICYLTY 1310.5150 133.9600 0.0000 ORIGIN $0.0-1.0473$

ZHEMICYL+Y $010.5220 \quad 143.9650 \quad 0.0000$ ORIGIN $0.0-1.0473$

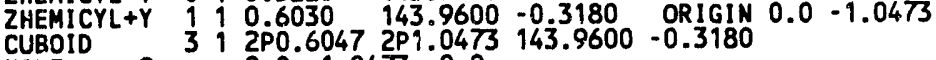

HOLE $9 \quad 0.01 .04730 .0$

$\begin{array}{lllll}\text { HOLE } & 17 & 0.0004 & 0.0 & 0.0 \\ \text { HOLE } & 27 & -0.6017 & 0.0 & 0.0\end{array}$ UNIT 43 COM $=$ CUBOID BUILT AROUND UNIT 12 WITH UNITS $1018 \& 28$ ADDED AS HOLES' 2HEMICYL+Y $23 i 0.5150 \quad 153.3400143 .9000$ ORIGIN $0.0-1.0473$

ZHEMICYLYY $010.5220 \quad 155.8040143 .9600$ ORIGIN $0.0-1.0473$

$Z$ HEMICYL+Y I $0.6030 \quad 156.1220143 .9600$ ORIGIN $0.0-1.0473$

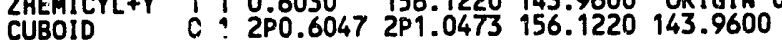


$\begin{array}{lllrl}\text { HOLE } & 10 & 0.0 & 1.0473 & 0.0 \\ \text { HOLE } & 18 & 0.6047 & 0.0 & 0.0 \\ \text { HOLE } & 28 & -0.6047 & 0.0 & 0.0\end{array}$

UNIT 49

COM= CCUBOID BUILT AROUND UNIT 3 HITH UNITS $5 \& 23$ ADDED AS HOLES' ZHEMICYL +Y 2 \% $0.6030 \quad 143.9600-0.3180$ ORIGIN $0.0-1.0473$ CUBOID 312 PO.6047 2 P $1.0473143 .9600-0.3180$

HOLE $\quad 55 \quad 0.017 .04730 .0$

HOLE $23 \quad-0.6047 \quad 0.0 \quad 0.0$

COM='CUBOID BUILT AROUND UNIT 4 WITH UNITS $6 \& 24$ ADDED AS HOLES

ZHEMICYL+Y $2 \$ 0.6030$ 156.1220 143.9600 ORIGIN $0.0-1.0473$

CUBOID O 12 PO.6047 $2 P 1.0473156 .1220143 .9600$

HOLE $\quad 26 \quad 0.001 .04730 .0$

UNIT 51

COM=' CUBOID BUILT AROUND UNIT 11 WITH UNITS $5 \& 19$ ADDED AS HOLES'

ZHEMICYLYY $13 \quad 130.5150 \quad 143.96000 .0 \quad$ ORIGIN $0.0-1.0473$

ZHEMICYL+Y $0110.5220 \quad 143.9600 \quad 0.0 \quad$ ORIGIN $0.0-1.0475$

ZHEMICYL+Y 110.06030 143.9600

$\begin{array}{lllllll}\text { CUBOID } & 3 & 1 & 2 P 0.6047 & 2 P 1.0473 & 143.9600 & -0.3180\end{array}$

$\begin{array}{lrrl}\text { HOLE } & 5 & 0.061 .0473 & 0.0 \\ \text { HOLE } & 19 & 0.60470 .0 & 0.0\end{array}$

UNIT 52

COM='CUBOID BUILT AROUND UNIT 12 WITH UNITS $6 \& 20$ ADDED AS HOLES'

ZHEMICYL+Y $2310.5150 \quad 153.3400143 .9600$ ORIGIN $0.0-1.0473$

ZHEMICYL+Y $010.5220 \quad 155.8040143 .9600$ ORIGIN $0.0-1.0473$

ZHEMICYL+Y 110.6030 156.1220 143.9600 ORIGIN $0.0 \cdot 1.0473$

CUBOID 012 P0.6047 2P1.0473 156.1220143 .9600

$\begin{array}{lllll}\text { HOLE } & 6 & 0.0 & 1.0473 & 0.0 \\ \text { HOLE } & 20 & 0.6047 & 0.0 & 0.0\end{array}$

UNIT 53

COM=ICUBOID BUILT AROUND UNIT 15 WITH UNITS $921 \& 25$ ADDED AS HOLES'

ZHEMICYL+Y $16: 10.5150 \quad 143.9600 \quad 0.0000$ ORIGIN $0.0-1.0473$

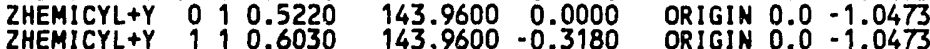

CUBOID 31 2P0.6047 2P1.0473 $143.9600-0.3180$

HOLE 9 9 0.01 .04730 .0

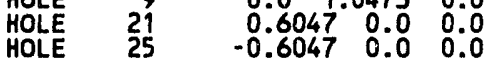

UNIT 54

COM $=$ 'CUBOID BUILT AROUND UNIT 16 WITH UNITS $1022 \& 26$ ADDED AS HOLES'

ZHEMICYL+Y $2610.5150 \quad 153.3400143 .9600$ ORIGIN $0.0-1.0473$

ZHEMICYL+Y O $0.5220 \quad 155.8040143 .9600$ ORIGIN $0.0-1.0473$

ZHEMICYL+Y 110.6030 156.1220 143.9600 ORIGIN $0.0-1.0473$

CUBDID 012 PP0.6047 2P1.0473 156.1220 143.9600

HOLE $10 \quad 0.01 .04730 .0$

$\begin{array}{lllll}\text { HOLE } & 22 & 0.6047 & 0.0 & 0.0 \\ \text { HOLE } & 26 & -0.6047 & 0.0 & 0.0\end{array}$

UNIT 55

COM=ICUBOID BUILT AROUND UNIT 15 WITH UNITS $921 \& 27$ ADDEC AS HC'. :S'

ZHEMICYL+Y $1610.5150 \quad 143.9600 \quad 0.0000$ ORIGIN $0.0-1.0473$

ZHEMICYL+Y $110.52030 \quad 143.9600 \quad 0.0000$ ORIGIN $0.0-1.0473$

CUBOID $312 \mathrm{P} 0.65472 \mathrm{ZP} 1.0473143 .9600-0.3180$

HOLE 9 O 0.004 .04730 .0

HOLE $21 \quad 0.6047 \quad 0.0 \quad 0.0$

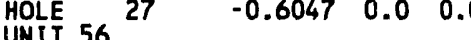

COM=ICUBOID BUILT AROUND UNIT 16 HITH UNITS $1022 \& 28$ ADDED AS HOLES'

ZHEMICYL+Y $2610.5150 \quad 153.3400143 .9600$ ORIGIN $0.0-1.0473$

ZHEMICYL+Y O $10.5220 \quad 155.8040143 .9600$ ORIGIN $0.0-1.0473$

ZHEMICYL+Y $110.6030 \quad 156.1220143 .9600$ ORIGIN $0.0-1.0473$

CUBOID 012 PO.6047 2P1.0473 156.1220 143.9600

HOLE $10 \quad 0.01 .04730 .0$

$\begin{array}{lllll}\text { HOLE } & 22 & 0.6047 & 0.0 & 0.0 \\ \text { HOLE } & 28 & -0.6047 & 0.0 & 0.0\end{array}$

UNIT 57

COM =' CUBOID BUILT AROU:S UNIT 15 HITH UNITS $919 \& 27$ ADDED AS HOLES'

ZHEMICYL+Y $1610.5150 \quad 143.96000 .0000$ ORIGIN $0.0-1.0473$

$143.9600 \quad 0.0000$ ORIGIN $0.0-1.0475$

ZHEMICYL+Y 1 i $0.6030 \quad 143.9600-0.3180$ ORIGIN $0.0-1.0473$

HOLE

$312 P 0.60472 P 1.0473143 .9600-0.3180$

ORIGIN $0.0-1.0473$

$19 \quad 0.6047^{\circ} 0.00$

$\begin{array}{lllll}H O L E & 27 & -0.604 & 0.0 & 0.0\end{array}$

UNIT 58

COM $=$ CUUBOID BUILT AROUND UNIT 16 WITH UNITS $1020 \& 28$ ADDED PS HOLES'

ZHEMICYL+Y $26 \quad 0.5150 \quad 153.3400143 .9600$ ORIGIN $0.0-1.0473$

ZHEMICYL+Y $0110.5220 \quad 155.8040143 .9600$ ORIGIN $0.0-1.0473$

ZHEMICYL+Y $110.6030 \quad 156.1220143 .9600$ ORIGIN $0.0-1.0473$

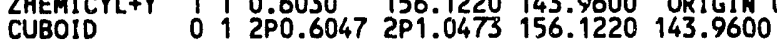


$\begin{array}{lllll}\text { HOLE } & 10 & 0.0 & 1.0473 & 0.0 \\ \text { HOLE } & 20 & 0.6047 & 0.0 & 0.0 \\ \text { HOLE } & 28 & -0.6047 & 0.0 & 0.0\end{array}$

UNIT 59

COM='CUBOID BUILT AROUND UNIT 11 WITH UNITS $5 \& 25$ ADDED AS HOLES'

ZHEMICYL+Y $131310.5150 \quad 943.96000 .0$ ORIGIN $0.0-1.0473$

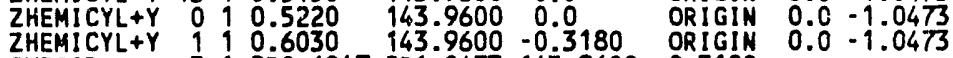

$\begin{array}{lllllll}\text { ZHEMICYL+Y } & 1 & 1 & 0.6030 & 143.9600 & -0.3180 & \text { ORIGIN } \\ \text { CUBOID } & 3 & 1 & 2 P 0.6047 & 2 P 1.0473 & 143.9600 & -0.3180\end{array}$

$\begin{array}{lllll}\text { HOLE } & 5 & 0.0 & 1.0473 & 0.0 \\ \text { HOLE } & 25 & -0.6047 & 0.0 & 0.0\end{array}$

COM= CUBOID BUILT AROUND UNIT 12 HITH UNITS 6 \& 26 ADDED AS HOLES

ZHEMICYL+Y $23 \quad 0.5150 \quad 153.3400143 .9600$ ORIGIN $0.0-1.0473$

ZHEMICYL+Y $010.5220 \quad 155.8040143 .9600$ ORIGIN $0.0-1.0473$

ZHEMICYL+Y 1910.6030 156.1220 143.9600 ORIGIN $0.0-1.0473$

$012 \mathrm{P} 0.60472 \mathrm{P} 1.0473156 .1220143 .9600$

$\begin{array}{lllll}\text { HOLE } & 6 & 0.0 & 1.0473 & 0.0 \\ \text { HOLE } & 26 & -0.6047 & 0.0 & 0.0\end{array}$

UNIT 61

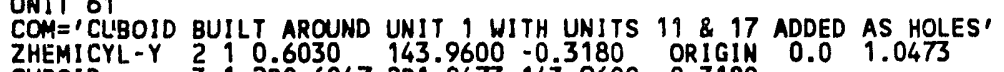
CUBOID $312 P 0.60472 P 1.0473143 .9600-0.3180$

$\begin{array}{llll}\text { HOLE } & 11 & 0.0-1.0473 & 0.0 \\ \text { HOLE } & 17 & 0.60470 .0 & 0.0\end{array}$

UNIT $6217 \quad 0.60470 .0 \quad 0.0$

COM=ICUBOID BUILT AROUND UNIT 2 WITH UNITS $12 \& 18$ ADDED AS HOLES'

ZHEMICYL-Y $210.6030 \quad 156.1220143 .9600$ ORIGIN 0.01 .0473

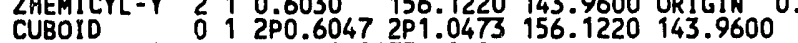

$\begin{array}{lllll}\text { HOLE } & 12 & 0.0-1.0473 & 0.0 \\ \text { HOLE } & 18 & 0.6047 & 0.0 & 0.0\end{array}$

UNIT 63

COM='CUBOID BUILT AROUND UNIT 5 WITH UNITS $1521 \& 23$ ADDED AS HOLES'

ZHEMICYL-Y 1310.5150 143.9600 10.0000 ORIGIN 0.001 .0473

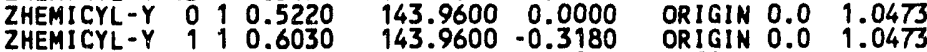

CUBOID 312 P0.6047 2P1.0473 $143.9600-0.3180$

HOLE 15 O $0.0-1.04730 .0$

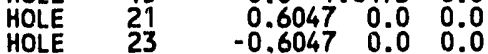

UNIT 64

COM $=$ 'CUBOID BUILT AROUND UNIT 6 WITH UNITS $1622 \& 24$ ADDED AS HOLES'

ZHEMICYL-Y $2310.5150 \quad 153.3400143 .9600$ ORIGIN 0.01 .0473

ZHEMICYL-Y $0110.5220 \quad 155.8040143 .9600$ ORIGIN 0.01 .0473

ZHEMICYL-Y $110.6030 \quad 156.1220143 .9600$ ORIGIN 0.01 .0473

CUBOID $012 P 0.60472 P 1.0473156 .1220143 .9600$

HOLE $16 \quad 0.0-1.04730 .0$

$\begin{array}{lllll}\text { HOLE } & 22 & 0.6047 & 0.0 & 0.0 \\ \text { HOLE } & 24 & -0.6047 & 0.0 & 0.0\end{array}$

\section{UNIT 65}

COM=' CUBOID BUILT AROUND UNIT 7 WITH UNITS 1521 \& 27 ADDED AS HOLES'

ZHEMICYL-Y $1410.5150 \quad 143.9600 \quad 0.0000$ ORIGIN 0.01 .0473

2HEMICYL-Y $010.5220 \quad 143.9600 \quad 0.0000$ ORIGIN $0.0 \quad 1.0473$

ZHEMICYL-Y $1110.6030 \quad 143.9600-0,3180$ ORIGIN $0.0 \quad 1.0473$

CUBOID 312 P0.6047 2P1.0473 $143.9600-0.3180$

HOLE $15 \quad 0.0-1.04730 .0$

$\begin{array}{lllll}\text { HOLE } & 21 & 0.6047 & 0.0 & 0.0 \\ \text { HOLE } & 27 & -0.6047 & 0.0 & 0.0\end{array}$

UNIT 66

COM $=$ 'CUBOID BUILT AROUND UNIT 8 WITH UNITS $1622 \& 28$ ADDED AS HOLES' ZHEMICYL-Y 24 $10.5150 \quad 153.3400143 .9600$ ORIGIN 0.001 .0473

ZHEMICYL-Y $010.5220 \quad 155.8040143 .9600$ ORIGIN 0.01 .0473

ZHEMICYL-Y 110.6030 156.1220 143.9600 ORIGIN 0.01 .0473

CUBOID 012 P0.6047 2P1.0473 156.1220143 .9600

HOLE $16 \quad 0.0-1.04730 .0$

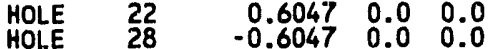

UNIT 67

COM=ICUBOID BUILT AROUND UHIT 5 WITH UNITS $1517 \& 27$ ADDED AS HOLES' ZHEMICYL-Y 13 \% $0.515 \mathrm{C} \quad 143.9600 \quad 0.0000$ ORIGIN 0.01 .0475

ZHEMICYL-Y $010.5220 \quad 143.96000 .0000$ ORIGIN $0.0 \quad 1.0475$

ZHEMICYL-Y $110.6030 \quad 143.9600-0.3180$ ORIGIN $0.0 \quad 1.0473$

CUBOID 312 PP0.6047 $2 P 1.0473143 .9600-0.3180$

HOLE 15 0.0 15.04730 .0

$\begin{array}{lllll}\text { HOLE } & 17 & 0.6047 & 0.0 & 0.0 \\ \text { HOLE } & 27 & -0.6047 & 0.0 & 0.0\end{array}$

UNIT 68

COM=' CUBOID BUILT AROUND UNIT 6 HITH LWITS 16 io \& 26 AOUDED AS HULLES

ZHEMICYL-Y 2310.5150 153.3400 143.9600 ORIGIN 0.01 .0473

ZHEMICYL-Y $010.5220 \quad 155.8040143 .9600$ ORIGIN 0.0 1.0473

ZHEMICYL-Y $110.5030 \quad 156.1220143 .9600$ ORIGIN $0.0 \quad 1.0473$

$\begin{array}{lllllll}\text { ZHEMICYL-Y } & 1 & 1 & 0.6030 & 156.1220 & 143.9600 & \text { ORIGIN } \\ \text { CUBOID } & 0 & 1 & 2 \mathrm{P} 0.6047 & 2 \mathrm{P} 1.0473 & 156.1220 & 143.9600\end{array}$ 


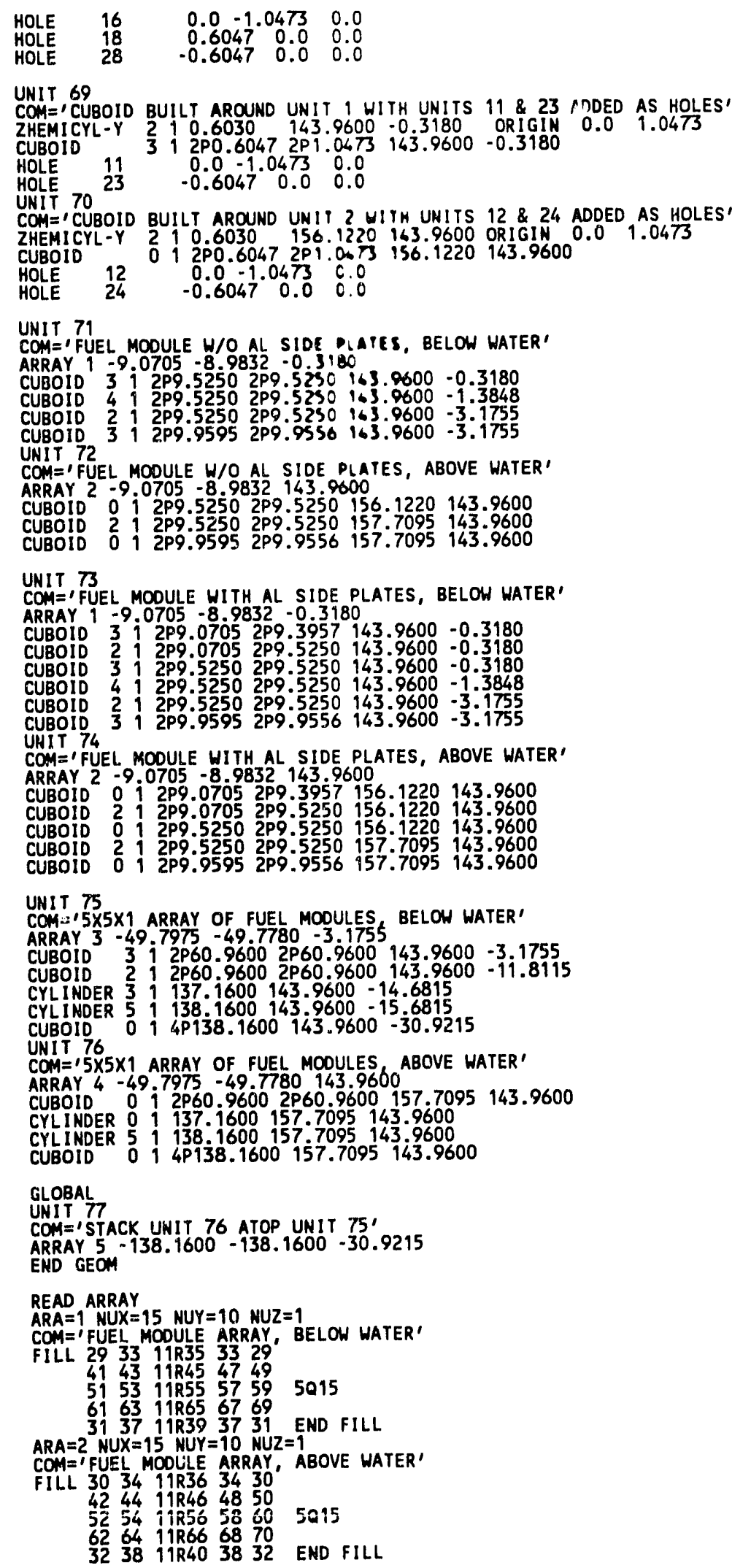


ARA $=3$ NUX $=5$ MUY $=5$ MUZ $=1$

COM=IARRAY OF FUEL MOOULES BELOW WATER'

FILL.

$$
\begin{aligned}
& 5 R 71 \\
& 71 \text { SRT3 } 71 \\
& 5 R 71 \\
& 71 \text { SRT3 } 71 \\
& 5 R 71 \text { END FILL }
\end{aligned}
$$

ARA $=4$ NUX $=5$ NUY $=5$ NUZ $=1$

ARA $=4$ NOX $=5$ NOY FUEL MOOULES ABOVE WATER
FILL

$$
\begin{array}{lll} 
& 5 R 72 \\
3 R 74 & 72 \\
5 R 72 & \\
72 & 3 R 74 & \\
5 R 72 & & \\
& & \\
&
\end{array}
$$

ARA $=5$ NUX=1 NUY $=1$ NUZ $=2$

ARA $=5$ NUX $=1$ NUY $=1$ NUZ $=2$
COM $=1$ STACKING ABOVE WATER UNIT ATOP BELOW WATER UNIT' FILL 7576 END FILL

END ARRAY

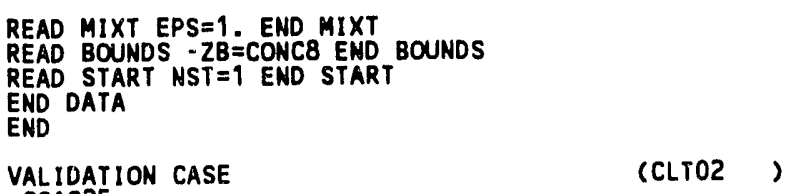

$$
\text { 27GROUPNDF4 LATTICECELL }
$$

BAW-1645-4 EXPIMT-2457, CASE C-2-C, DANCOFF FACTOR FOR EACH FUEL PIN

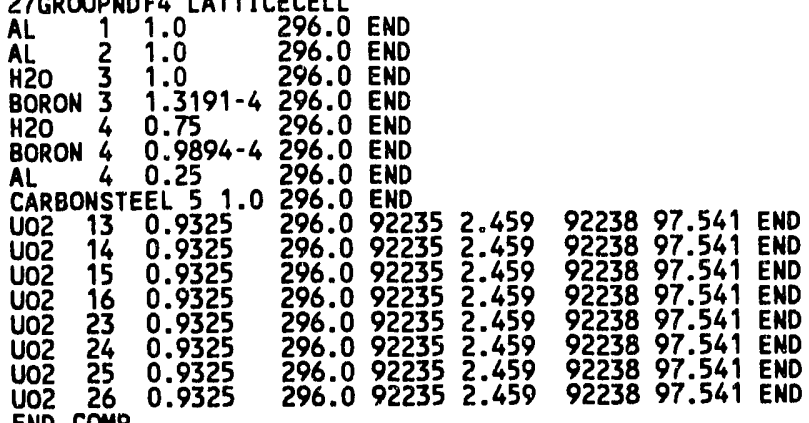

END COMP

TRIANGPITCH 1.20931 .03001631 .206011 .04400 END

MORE DATA RES $=13$ CYLINDER 0.5150 DAN (13) $=0.37809$

RES 14 CYLINDER 0.5150 DAN 14$)=0.50536$

RES $=15$ CYIINDER 0.5150 DAN (15) $=0.63264$

RES $=23$ CYLINDER 0.5150 DAN $(23)=0.46920$

RES $=24$ CYLINDER 0.5150 DAN $(24)=0.63217$

RES $=25$ CYLIMDER 0.5150 DAN $(25)=0.79514$

BAY-1645-4 EXPIMT-2457 CASE C-2-C DANCDFF FACTOR FOR EACH FUEL PIN

BAH-1645-4, EXP'MT - 2457, CASE C-2-C, DANCOF

READ PARAM

$\begin{array}{llllll}\text { UNIT } 1 & & & \\ \text { ZHEMICYL-Y } & 2 & 1 & 0.6030 & 104.8200 & -0.3180 \\ \text { UNIT } 2 & & & & \\ \text { ZHEMICYL-Y } & 2 & 1 & 0.6030 & 156.1220 & 104.8200 \\ \text { UNIT } 3 & 3 & & \\ \text { ZHEMICYL+Y } & 2 & 1 & 0.6030 & 104.8200 & -0.3180 \\ \text { UNIT } 4 & 4 & & 0.6030 & 156.1220 & 104.8200 \\ \text { ZHEMICYL+Y } & 2 & 1 & 0.6030 & 156.1220 & \end{array}$

UNIT 5

ZHEMICYL-Y $13 \quad 1 \quad 0.5150 \quad 104.8200 \quad 0.0$

ZHEMICYL-Y

ZHEMICYL-Y $110.6030 \quad 104.8200 \quad-0.3180$

UNIT 6

ZHEMICYL.Y $23,0.5150153 .3400 \quad 104.8200$

ZHEMICYL-Y $010.5220155 .8040 \quad 104.8200$

ZHEMICYL-Y 110.6030156 .1220104 .8200

UNEMT 7

UNIT Y

ZHEMICYL-Y 14 I $0.5150 \quad 104.82000 .0$

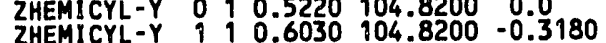

ZHEMICY

ZHEMICYL-Y $2410.5150153 .3400 \quad 104.8200$

ZHEMICYL-Y O $10.5220155 .8040 \quad 104.8200$

ZHEMICYLYY $190.6030 \quad 156.1220 \quad 104.8200$ 


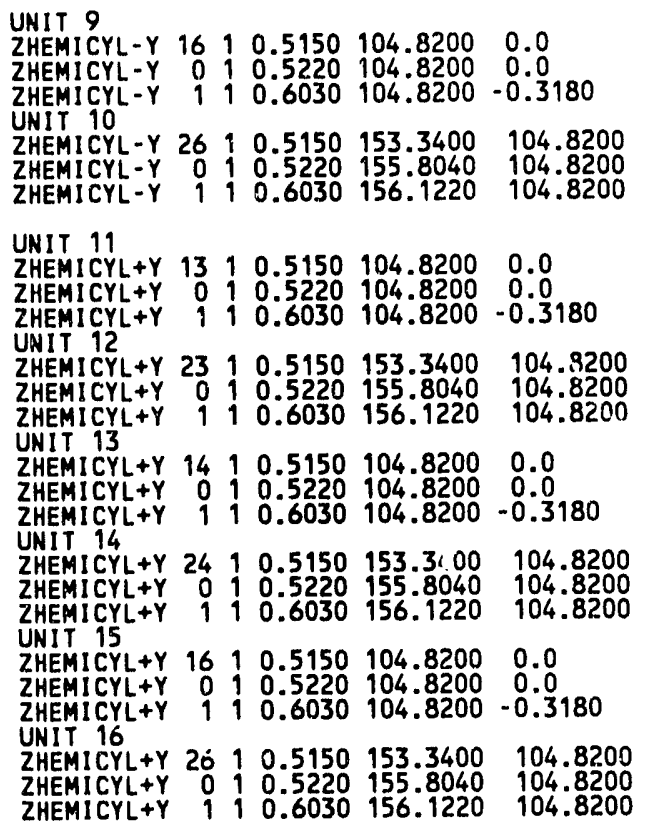

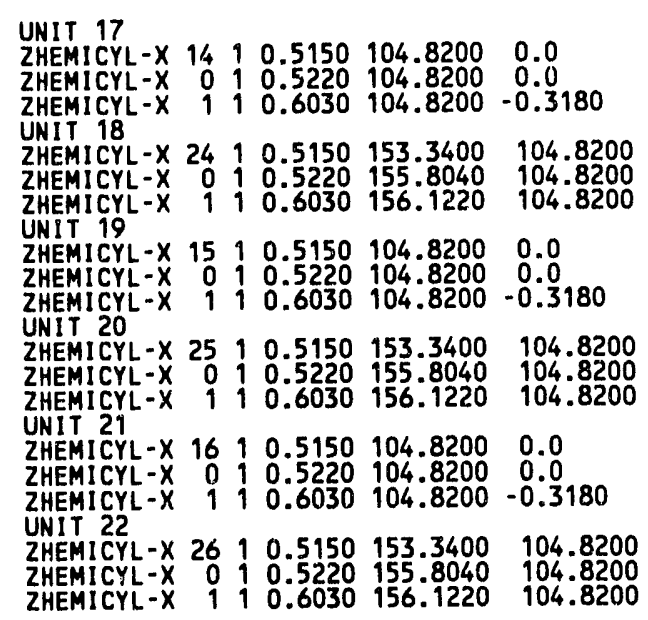

UNIT 23

ZHEMICYLL+X $14 \quad 1 \quad 0.5150 \quad 104.8200 \quad 0.0$

ZHEMICYL+X 010.5220104 .82000 .0

ZHEMICYL+X 11 1 $0.6030 \quad 104.8200 \quad-0.3180$

UNIT 24

ZHEMICYL+X $24 \quad 1 \quad 0.5150 \quad 153.3400 \quad 104.8200$

HHEMICYL $X$ O $19.5220155 .8040 \quad 104.8200$

ZHEMICYL+X $1110.6030156 .1220 \quad 104.8200$

ZHEMICYL+X $1510.5150 \quad 104.8200 \quad 0.0$

$\begin{array}{llllll}\text { ZHEMICYL+X } & 0 & 1 & 0.5220 & 104.8200 & 0.0 \\ & & 0.0 & \\ \text { ZHEMICYL +X } & 1 & 1 & 0.6030 & 104.8200 & -0.3180\end{array}$

ZHEMICYL $+X \quad 110.6030104 .3200-0.3180$

ZHEMICYL +X $25 \quad 1 \quad 0.5150 \quad 153.3400 \quad 104.8200$

ZHEMICYL +X 0 T $0.5220155 .8040 \quad 104.8200$

ZHEMICYL+X $110.6030 \quad 156.1220 \quad 104.8200$

UNIT 27

ZHEMICYL $+X 16 \quad 1 \quad 0.5150 \quad 104.8200 \quad 0.0$

ZHEMICYL+X 0 1 $0.5220104 .8200 \quad 0.0$

$\begin{array}{lllllll}2 H E M T L & & \end{array}$

UNIT 28

UNIT 28

$\begin{array}{llllll}\text { ZHEMICYLLX } & 26 & 1 & 0.5150 & 153.3400 & 104.8200 \\ \text { ZHEMICYL+X } & 1 & 0.5220 & 155.8040 & 104.8200\end{array}$

$\begin{array}{llllll}\text { ZHEMICYL+X } & 0 & 1 & 0.5220 & 55.8040 & 104.8200 \\ \text { ZHEMICYL+X } & 1 & 1 & 0.60 & 56.1220 & 104.8200\end{array}$

UNIT 29

COM $=$ 'CUBOID BUILT AROUND UNIT 1 '

ZHEMICYL-Y 210.6030

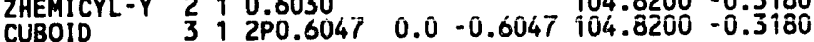


UNIT 30

COM=ICUBOID BUILT AROUND UNIT 2'

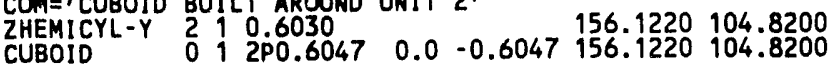
UNiT 31

COM $=$ CCUBOID BUILT AROUND UNIT 3 '

CHEMICYLIY 290.6030 UNIT 3' $104.8200 \cdot 0.3180$

$\begin{array}{llllllll}\text { ZHEMICYL+Y } & 2 & 1 & 0.6030 & & & & \\ \text { CUBOID } & 3 & 1 & 2 \mathrm{P} 0.6047 & 0.6047 & 0.0 & 104.8200 & -0.3180 \\ & & & 104.8200 & -0.3180\end{array}$

UNIT 32

COM=' CUBOID BUILT AROUND UNIT 4'

$\begin{array}{llllllll}\text { ZHEMICYL +Y } & 2 & 1 & 0.6030 & & 156.1220 & 104.8200 \\ \text { CUBOID } & 0 & 1 & 2 P 0.6047 & 0.6047 & 0.0 & 156.1220 & 104.8200\end{array}$

\begin{tabular}{|c|c|c|c|c|}
\hline \multicolumn{5}{|c|}{$0.0-0.6$} \\
\hline $\begin{array}{l}\text { UNIT } 34 \\
\text { ZHEMICYL-Y } \\
\text { ZHEMICYL-Y } \\
\text { ZHEMICYL-Y } \\
\text { CUBOID }\end{array}$ & $\begin{array}{rll}23 & 1 & 0.5150 \\
0 & 1 & 0.5220 \\
1 & 1 & 0.6030 \\
0 & 1 & 2 \mathrm{P} 0.6047\end{array}$ & $0.0-0.6047$ & $\begin{array}{l}153.3400 \\
155.8040 \\
156.1220 \\
156.1220\end{array}$ & $\begin{array}{l}104.8200 \\
104.8200 \\
104.8200 \\
104.8200\end{array}$ \\
\hline $\begin{array}{l}\text { UNIT } 35 \\
\text { ZHEMICYL-Y } \\
\text { ZHEMICYL-Y } \\
\text { ZHEMICYL-Y } \\
\text { CUBOID }\end{array}$ & $\begin{array}{rll}14 & 1 & 0.5150 \\
0 & 1 & 0.5220 \\
1 & 1 & 0.6030 \\
3 & 1 & 280.6047\end{array}$ & $\begin{array}{lll}0.0 & -0.6047\end{array}$ & $\begin{array}{l}104.8200 \\
104.8200 \\
104.8200 \\
104.8200\end{array}$ & $\begin{array}{l}0.0 \\
0.0 \\
-0.3180 \\
-0.3180\end{array}$ \\
\hline $\begin{array}{l}\text { UNIT } 36 \\
\text { ZHEMICYL-Y } \\
\text { ZHEMICYL-Y } \\
\text { ZHEMICYL-Y } \\
\text { CUBOID }\end{array}$ & $\begin{array}{rll}24 & 1 & 0.5150 \\
0 & 1 & 0.52220 \\
1 & 1 & 0.6030 \\
0 & 1 & 2 \mathrm{P} 0.6047\end{array}$ & $\begin{array}{lll}0.0 & -0.6047\end{array}$ & $\begin{array}{l}153.3400 \\
155.8040 \\
156.1220 \\
156.1220\end{array}$ & $\begin{array}{l}104.8200 \\
104.8200 \\
104.8200 \\
104.8200\end{array}$ \\
\hline $\begin{array}{l}\text { UNIT } 37 \\
\text { ZHEMICYL+YY } \\
\text { ZHEMICYL+.Y } \\
\text { ZHEMICYL+Y } \\
\text { CUBOID }\end{array}$ & $\begin{array}{rll}13 & 1 & 0.5150 \\
0 & 1 & 0.52200 \\
1 & 1 & 0.6030 \\
3 & 1 & 280.6047\end{array}$ & $0.6047 \quad 0.0$ & $\begin{array}{l}104.8200 \\
104.8200 \\
104.8200 \\
104.8200\end{array}$ & $\begin{array}{l}0.0 \\
0.0 \\
-0.3180 \\
-0.3180\end{array}$ \\
\hline $\begin{array}{l}\text { ZHEMIC } \\
\text { ZHEMI CY } \\
\text { ZHEMI }\end{array}$ & $\begin{array}{rll}23 & 1 & 0.5150 \\
0 & 1 & 0.5220 \\
1 & 1 & 0.6030 \\
0 & 1 & 2 P 0.6047\end{array}$ & $0.6047 \quad 0.0$ & $\begin{array}{l}153.3400 \\
155.8040 \\
156.1220 \\
156.1220\end{array}$ & $\begin{array}{l}104.8200 \\
104.8200 \\
104.8200 \\
104.8200\end{array}$ \\
\hline $\begin{array}{l}\text { ZHEM } \\
\text { ZHEM } \\
\text { ZHEM }\end{array}$ & $\begin{array}{rll}14 & 1 & 0.5150 \\
0 & 1 & 0.5220 \\
1 & 1 & 0.6030 \\
3 & 1 & 2 \mathrm{P} 0.6047\end{array}$ & $0.6047 \quad 0.0$ & $\begin{array}{l}104.8200 \\
104.8200 \\
104.8200 \\
104.8200\end{array}$ & $\begin{array}{l}0.0 \\
0.0 \\
-0.3180 \\
-0.3180\end{array}$ \\
\hline $\begin{array}{l}\text { ZHEMICYL+Y } \\
\text { ZHEMICYL }+Y \\
\text { ZHEMICYL } \\
\text { CUBOID }\end{array}$ & $\begin{array}{rll}24 & 1 & 0.5150 \\
0 & 1 & 0.5220 \\
1 & 1 & 0.6030 \\
0 & 1 & 2 p 0.6047\end{array}$ & $0.6047 \quad 0.0$ & $\begin{array}{l}153.3400 \\
155.8040 \\
156.1220 \\
0156.1220\end{array}$ & $\begin{array}{l}104.8200 \\
104.8200 \\
104.8200 \\
104.8200\end{array}$ \\
\hline
\end{tabular}

UNIT 41

CON=' CUBOID BUILT AROUND UNIT 3 HITH UNITS $5 \& 17$ ADDED AS HOLES'

ZHEMICYL+Y 210.6030 104.8200 -0.3180 ORIGIN $0.0-1.0473$

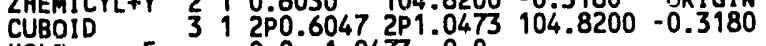

$\begin{array}{lllll}\text { HOLE } & 5 & 0.0 & 1.0473 & 0.0 \\ \text { HOLE } & 17 & 0.6047 & 0.0 & 0.0\end{array}$

UNIT 42 2 OOD

COM $=$ CUBOID BUILT AROUND UNIT 4 HITH UNITS $6 \& 18$ ADDED AS HOLES

ZHEMICYL+Y $210.6030 \quad 156.1220104 .8200$ ORIGIN $0.0-1.0473$

CUBOID $012 \mathrm{P} 0.60472 \mathrm{P} 1.0473 \quad 156.1220104 .8200$

$\begin{array}{llllll}\text { HOLE } & 6 & 0.0 & 1.0473 & 0.0 \\ \text { HOLE } & 18 & 0.6047 & 0.0 & 0.0\end{array}$

UNIT 43

COM=I CUBOID BUILT AROUND UNIT 11 WITH UNITS $921 \& 23$ ADDED AS HOLES'

2HEMICYL+Y $1310.5150 \quad 104.8200 \quad 0.0000$ ORIGIN $0.0-1.0473$

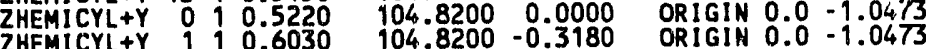

$\begin{array}{lllllll}2 \text { HEMICYL+Y } & 1 & 1 & 0.6030 & 104.8200 & -0.3180 & \text { ORIGIN } \\ & 3 & 1 & 2 \mathrm{P} 0.6047 & 2 \mathrm{P} 1.0473 & 104.8200 & -0.3180\end{array}$

HOLE 9 O 0.01 .04730 .0

HOLE $21 \quad 0.60470 .0000$

HOLE 44

UNIT 44 POID BUILT AROUND UNIT 12 HITH UNITS $1022 \& 24$ ADDED AS HOLES'

ZHEMICYL+Y $2310.5150 \quad 153.3400104 .8200$ ORIGIN $0.0-1.0473$

2HEMICYL+Y 010.5220 156.8040 104.8200 ORYGIN $0.0-1.0473$

ZHEMICYL+Y 1110.6030 156.1220 104.8200 ORYGIN

CUBOID $012 P 0.60472 P 1.0473156 .1220104 .8200$

HOLE $10 \quad 0.01 .04730 .0$

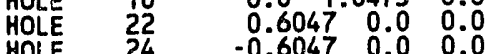


UNIT 45

COM='CUBOID BUILT AROUND UNIT 13 WITH UNITS $921 \& 27$ ADDED AS HOLES'

ZHEMICYL +Y $1410.5150 \quad 104.8200 \quad 0.0000$ ORIGIN $0.0-1.0473$

ZHEMICYL+Y $010.5220 \quad 104.82000 .0000$ ORIGIN $0.0=1.0473$

$\begin{array}{lllllll}\text { ZHEMICYL+Y } & 1 & 1 & 0.6030 & 104.8200 & 0.3180 & \text { ORIGIN } \\ \text { CUBOID } & 3 & 1 & 2 \mathrm{P} 0.6047 & 0.041 .0473 & 104.8200 & -0.3180\end{array}$

CUBOID \& 312 PO.6047 2 P 1.0473

$\begin{array}{lllll}\text { HOLE } & 21 & 0.6047 & 0.0 & 0.0 \\ \text { HOLE } & 27 & -0.6047 & 0.0 & 0.0\end{array}$

UNIT 46

COM='CUBOID BUILT AROUND UNIT 14 WITH UNITS $1022 \& 28$ ADDED AS HOLES'

ZHEMICYL+Y $24 \quad 10.5150 \quad 153.3400104 .8200$ ORIGIN $0.0-1.0473$

ZHEMICYLYY O $10.5220 \quad 155.8040104 .8200$ ORIGIN $0.0-1.0475$

ZHEMICYLTY 110.6030 156.1220 104.8200 ORIGIN 0.0

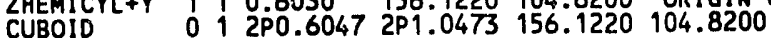

HOLE $10 \quad 0.01 .04730 .0$

$\begin{array}{lllll}\text { HOLE } & 10 & 0.6047 & 0.0 & 0.0 \\ \text { HOLE } & 22 & 0.604 & 0.0 & 0.0\end{array}$

UNIT 47

COM='CUBOID BUILT AROUND UNIT 11 WITH UNITS $917 \& 27$ ADDED AS HOLES'

ZHEMICYL +Y $1310.5150 \quad 104.8200 \quad 0.0000$ ORIGIN $0.0-1.04 \%$

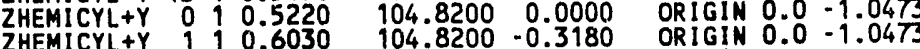

CUBOID 312 P0.6047 2P1.0473 $104.8200-0.3180$

HOLE $9 \quad 0.01 .04730 .0$

HOLE $17 \quad 0.604700 .00 .0$

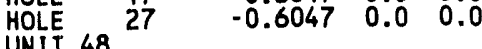

COM=1CUBOID BUILT AROUND UNIT 12 UITH UNITS $1018 \& 28$ ADDED AS HOLES

ZHEMICYL +Y 2310.5150 153.3400 104.8200 ORIGIN $0.0-1.0473$

2HEMICYLY $2310.5250 \quad 155.8040104 .8200$ ORIGIN $0.0-1.0473$

ZHEMICYL+Y 1.5300156 .1220104 .8200 ORIGIN $0.0-1.0473$

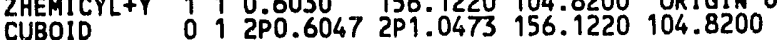

HOLE $10 \quad 0.01 .04730 .0$

$\begin{array}{lllll}\text { HOLE } & 18 & 0.6047 & 0.0 & 0.0 \\ \text { HOLE } & 28 & -0.6047 & 0.0 & 0.0\end{array}$

UNIT 49

COM $=$ ' CUBOID BUILT AROUND UHIT 3 WITH UNITS $5 \& 23$ ADDED AS HOLES

COFEICYL +Y $10.6030104 .8200-0.3180$ ORIGIN $0.0-1.0473$

ZHEMICYL+Y $210.6030472 \mathrm{P} 1.0473 \quad 104.8200-0.3180$

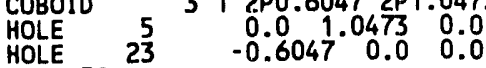

UNIT 50 O

COM='CUBOID BUILT AROUND UNIT 4 WITH UNITS $6 \& 24$ ADDED AS HOLES

ZHEMICYL+Y $210.6030 \quad 156.1220104 .8200$ ORIGIN $0.0-1.0473$

CUBOID $012 P 0.60472 P 1.0473156 .1220104 .8200$

$\begin{array}{lrrrr}\text { HOLE } & 6 & 0.0 & 1.0473 & 0.0 \\ \text { HOLE } & 24 & -0.6047 & 0.0 & 0.0\end{array}$

UNIT 51 1

COM $=$ 'CUBOID BUILT AROUND UNIT 110 WITH UNITS 5 \&

ZHEMICYL+Y $13130.5150 \quad 104.82000 .0 \quad$ ORIGIN $0.0-1.0475$

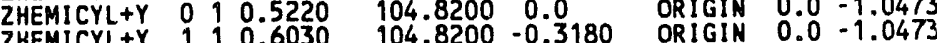

$\begin{array}{lllllll}\text { ZKEMICYL+Y } & 1 & 1 & 0.6030 & 104.8200 & -0.3180 & \text { ORIGIN } \\ \text { CUBOID } & 3 & 1 & 2 \mathrm{P} 0.6047 & 2 \mathrm{P} 1.0473 & 104.8200 & -0.3180\end{array}$

$\begin{array}{llll}\text { CUBOID } & 312 \mathrm{PO} .6047 & 2 \mathrm{P} 1.0473 \\ \text { HOLE } & 5.0 & 1.04730 .0\end{array}$

HOLE $19 \quad 0.60470 .0 \quad 0.0$

UNIT 52 2 DOMILT AROUND UNIT 12 WITH UNITS $6 \& 20$ ADDED AS HOLES'

ZHEMICYLTY $23,0.5150 \quad 153.3400104 .8200$ ORIGIN $0.0 \cdot 1.0473$

HEMICYLY $0.5220 \quad 155.8040104 .8200$ ORIGIN $0.0-1.0473$

ZHEMICYL+Y $190.6030 \quad 156.1220104 .8200$ ORIGIN $0.0-1.0473$

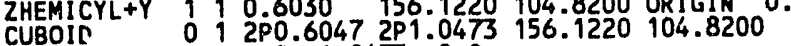

HOLE 6010.01 .04730 .0

UNIT 53 .

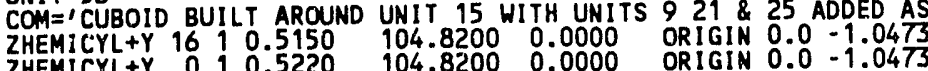

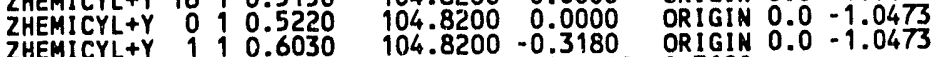

CUBOID 312 PP0.6047 2P1.0473 $104.8200-0.3180$

HOLE $90.0 \quad 1.04730 .0$

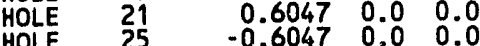

HNIT 5425

WNIT 54 BOID BUILT AROUND UNIT 16 WITH UNITS $1022 \& 26$ ADDED AS HOLES

COM=V

ZHEMICYL+Y 2610.5150

ZHEMICYL+Y $0110.5220 \quad 155.8040104 .8200$ ORIGIN $0.00=1.0473$

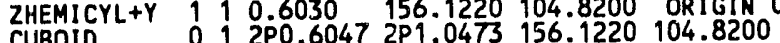

$\begin{array}{lllll}\text { CUBOID } & 0 & 12 P 0.6047 & 2 P 1.0473 \\ \text { HOLE } & 10 & 0.01 .0473 & 0.0\end{array}$

$\begin{array}{lllll}\text { HOLE } & 10 & 0.6047 & 047 & 0.0 \\ \text { HOLE } & 22 & 0.6047 & 0.0 & 0.0 \\ \text { HOLE } & 26 & -0.6047 & 0.0 & 0.0\end{array}$ 
UNIT 55

COM=I CUBOID BUILT AROUND UNIT 15 WITH UIVITS $921 \& 27$ ADDED AS MOLES

ZHEMICYL+Y $16,0.5150$ 104.8200 0.0000 ORIGIN $0.0-1.0473$

ZHEMICYL+Y $10.5220 \quad 104.82000 .0000$ ORIGIN $0.0-1.0473$

ZHEMICYL 1 1 $10.6030 \quad 104.8200-0.3180$ ORIGIN $0.0-1.0473$

CUBOID $312 P 0.6047$ 2P1.0473 $104.8200-0.3180$

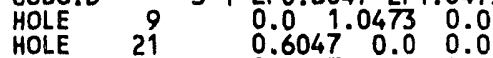

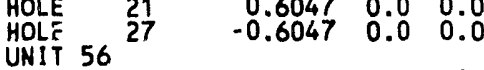

COM='CUBOID BUILT AROUND UNIT 16 WITH UNITS $1022 \& 28$ ADDED AS HOLES'

ZHEMICYL+Y $2610.5150 \quad 153.3400104 .8200$ ORIGIN $0.0-1.0473$

ZHEMICYL+Y O $10.5220 \quad 155.8040104 .8200$ ORIGIN $0.0-1.0473$

ZHEMICYL+Y 110.6030 156.1220 104.8200 ORIGIN $0.0-1.0473$

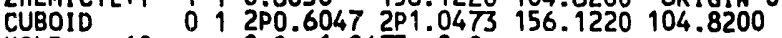

HOLE 10 O 0.01 .04730 .0

$\begin{array}{lllll}\text { HOLE } & 22 & 0.6047 & 0.0 & 0.0 \\ \text { HOLE } & 28 & 0.6047 & 0.0 & 0.0\end{array}$

UNIT 57

COM=I CUBOID BUILT AROUND UNIT 15 WITH UNITS 9 i9 \& 27 ADDED AS HOLES'

ZHEMICYLYY 1610.5150 104.8200 0.0000 CRIGIN $0.0-1.0473$

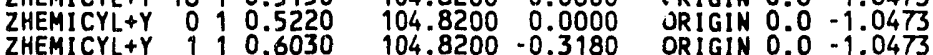

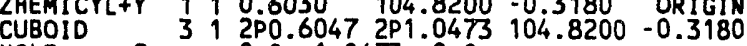

HOLE 9 O 0.011 .04730 .0

$\begin{array}{lllll}\text { HOLE } & 19 & 0.6047 & 0.0 & 0.0 \\ \text { HOLE } & 27 & -0.6047 & 0.0 & 0.0\end{array}$

HOLE $58^{27}$

COM=I CUBOID BUILT AROUND UNIT 16 HITH UNITS $1020 \& 28$ ADDED AS HOLES'

ZHEMICYL+Y $26 \div 0.5150 \quad 153.3400104 .8200$ ORIGIN $0.0-1.0473$

ZHEMICYL 20 Y $0.5220 \quad 155.8040104 .8200$ ORIGIN $0.0-1.0473$

2HEMICYL+Y $190.6030 \quad 156.1220104 .8200$ ORIGIN $0.0-1.0473$

CUBOID 10 O 12 2P0.6047 2 P1.0473 156.1220104 .8200

HOLE $10 \quad 0.011 .04730 .0$

$\begin{array}{lllll}\text { HOLE } & 20 & 0.6047 & 0.0 & 0.0 \\ \text { HOLE } & 28 & -0.6047 & 0.0 & 0.0\end{array}$

UNIT 59

COM=' CUBOID BUILT AROUND UNIT 11 WITH UNITS $5 \approx 25$ ADDED AS HOLES'

ZHEMICYL+Y $1310.5150 \quad 104.82000 .0$ ORIGIN $0.0 \% 1.0473$

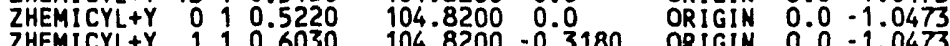

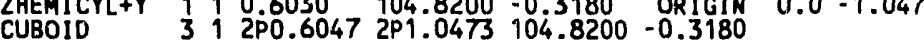

HOLE 550.001 .04730 .0

HOLE $25 \quad-0.60470 .0 \quad 0.0$

COM $=$ CCUBOID BUILT AROUND UNIT 12 HITH UNITS $6 \& 26$ ADDED AS HOLES'

ZHEMICYL+Y $2310.5150 \quad 153.3400104 .8200$ ORIGIN $0.0-1.0473$

ZHEMICYL+Y O $10.5220 \quad 155.8040104 .8200$ ORIGIN $0.0-1.0473$

ZHEMICYL+Y 119.6030 156.1220 104.8200 ORIGIN $0.0-1.0473$

CUBOID O $12 P 0.60472 P 1.0473156 .1220104 .8200$

$\begin{array}{lrrrr}\text { HOLE } & 6 & 0.0 & 1.0473 & 0.0 \\ \text { HOLE } & 26 & -0.6047 & 0.0 & 0.0\end{array}$

UNIT 61

COM $=$ 'CUBOID BUILT AROUND UNIT 1 WITH UNITS $11 \& 17$ ADDED AS HOLES'

ZHEMICYL-Y $210.6030 \quad 104.8200-10.3180$ ORIGIN 0.01 .0473

$\begin{array}{llll}\text { CUBOID } & 3 & 1 \\ 2 P 0.6047 & 2 \mathrm{P} 1.0473 & 104.8200 & -0.3180\end{array}$

HOLE 11 (1) $0.07-1.0473 .0 .0$

$\begin{array}{lll}\text { HOLE } & 17 & 0.6047 \quad 0.0 \quad 0.0\end{array}$

COM= $=$ CUBOID BUILT AROUND UNIT 2 HITH UNITS $12 \& 18$ ADDED AS HOLES'

ZHEMICYL-Y $210.6030 \quad 156.1220104 .8200$ ORIGIN $0.0 \quad 1.0473$

CUBOID 012 PO.6047 2P1.0473 156.1220104 .8200

$\begin{array}{lllll}\text { HOLE } & 12 & 0.0 & -1.0473 & 0.0 \\ \text { HOLE } & 18 & 0.6047 & 0.0 & 0.0\end{array}$

UNIT 63

COM $=$ 'CUBOID BUILT AROUND UNIT 5 WITH UNITS $1521 \& 23$ ADDED AS HOLES

ZHEMICYL-Y $1310.5150 \quad 104.8200 \quad 0.0000$ ORIGIH $0.0 \quad 1.0473$

ZHEMICYL-Y $010.5220 \quad 104.8200 \quad 0.0000$ ORIGIN $0.0 \quad 1.0473$

ZHEMICYL-Y $110.6030 \quad 104.8200-0.3180$ ORIGIN $0.0 \quad 1.0473$

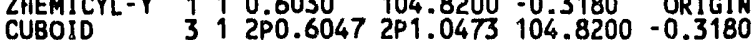

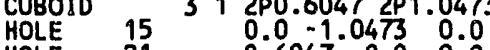

$\begin{array}{lllll}\text { HOLE } & 21 & 0.6047 & 0.0 & 0.0 \\ \text { HOLE } & 23 & -0.6047 & 0.0 & 0.0\end{array}$

UNIT 64

COM=' CUBOID BUILT AROUND UNIT 6 WITH UNITS $1622 \& 24$ ADDED AS HOLES'

ZHEMICYL-Y 2310.5150153 .3400104 .8200 ORIGIN 0.01 .0473

ZHEMICYL-Y $010.5220 \quad 155.8040104 .8200$ ORIGIN 0.01 .0473

ZHEMICYL-Y $1110.6030 \quad 156.1220104 .8200$ ORIGIN $0.0 \quad 1.0473$

CUBOID 012 P0.6047 2P1.0473 156.1220 104.8200

HOLE $16 \quad 0.0-1.04730 .0$

HOLE $22 \quad 0.60470 .00 .0$

$\begin{array}{lllll}\text { HOLE } & 24 & -0.6047 & 0.0 & 0.0\end{array}$ 


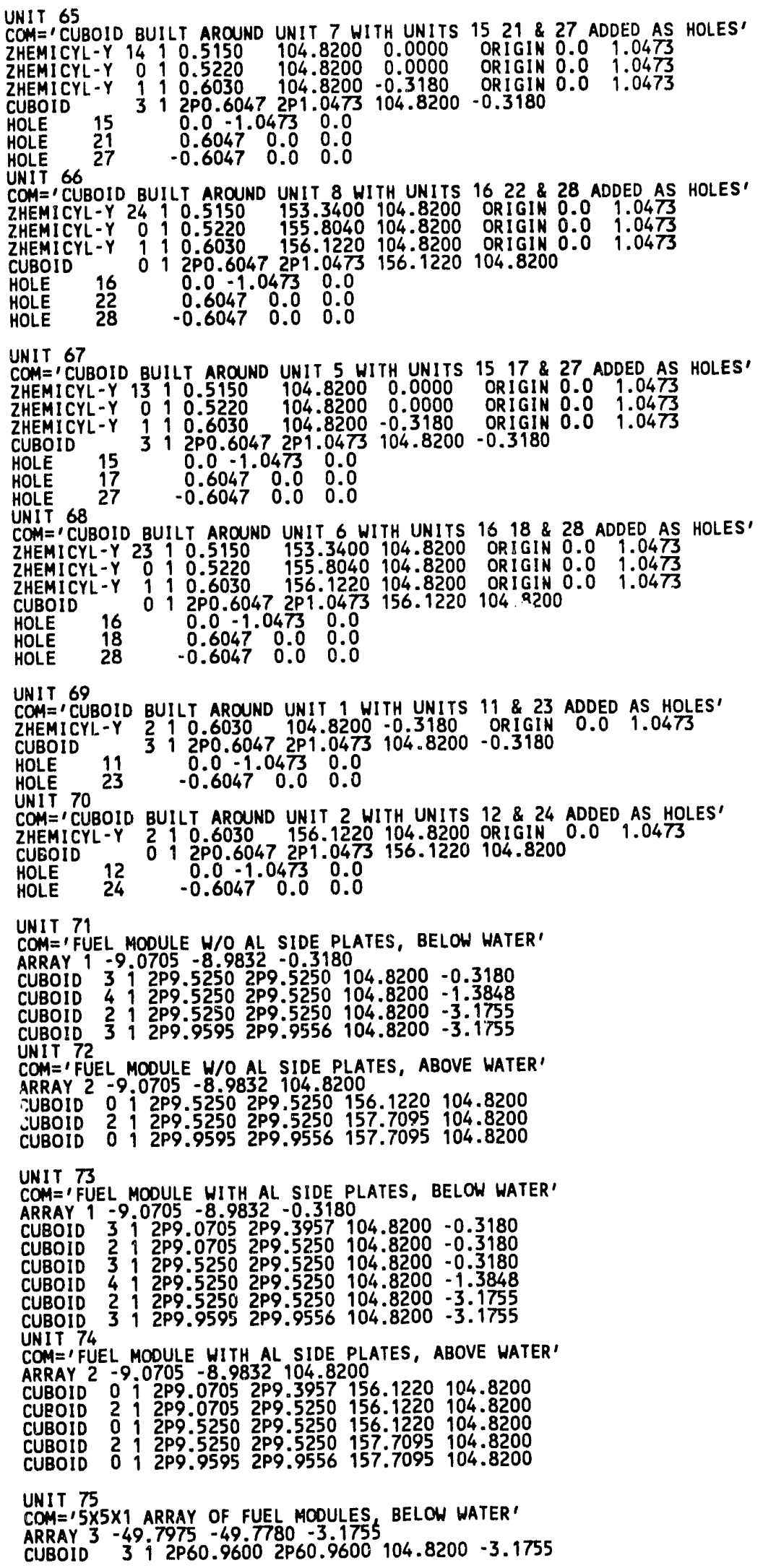




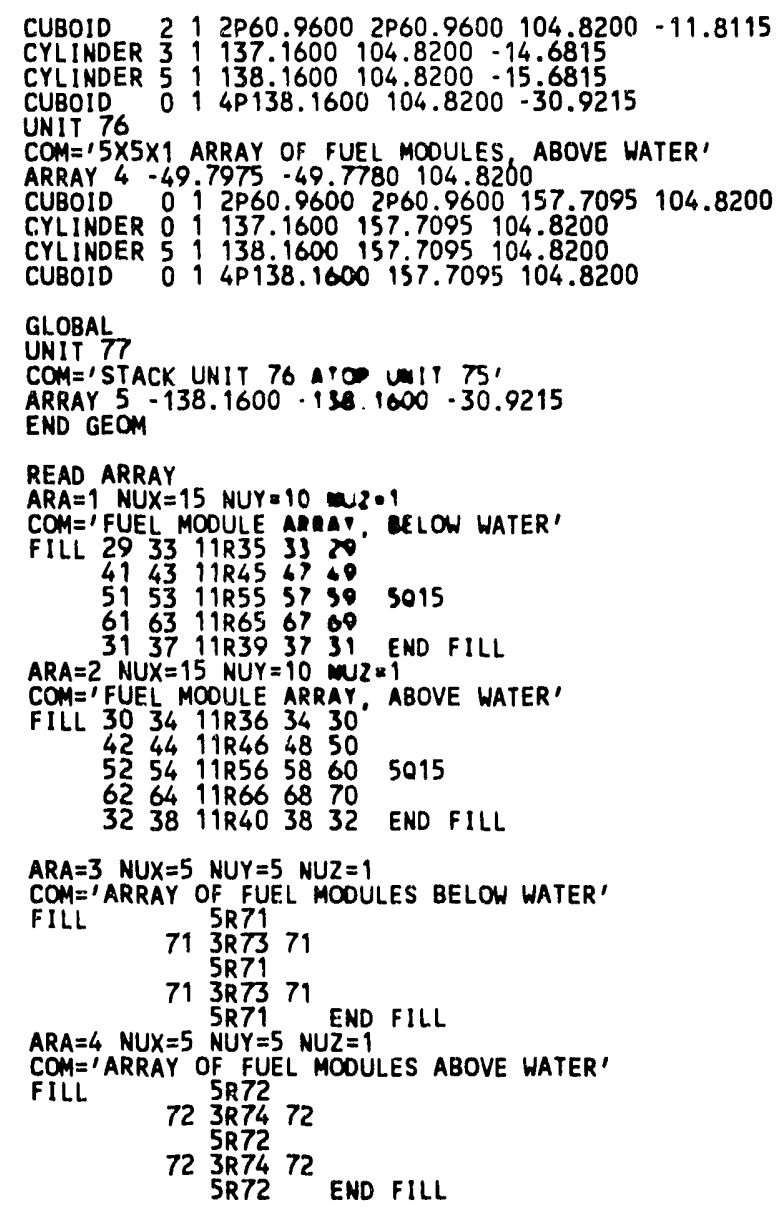

ARA $=5$ NUX $=1$ NUY $=1$ NUZ $=2$

COM $=$ ' STACKING ABOVE WATER UNIT ATOP BELOW WATER UNIT' FILL 7576 END FILL

END ARRAY

READ MIXT EPS $=1$. END MIXT

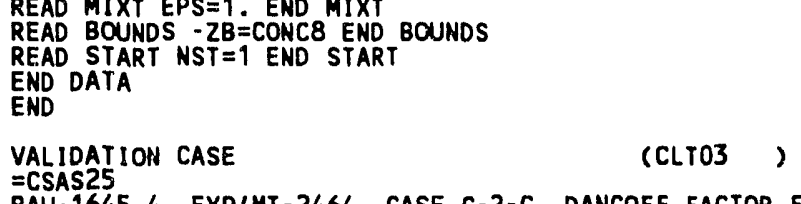

$=$ CSAS25

BAW-1645-4, EXP'MT-2464, CASE C-2-C, DANCOFF FACTOR FOR EACH FUEL PIN 27GROUPNDF4 LATTICECELI

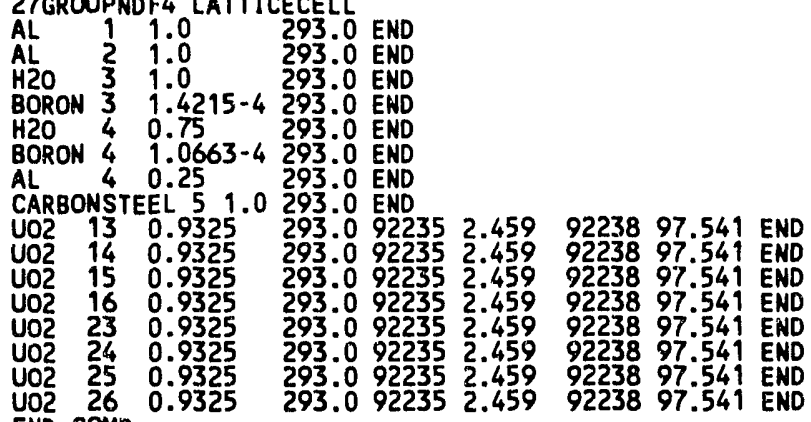

TRIANGPITCH 1.20931 .03001631 .206011 .04400 END

MORE DATA RES $=13$ CYLINDER 0.5150 DAN $\{33)=0.37808$

RES $=14$ CYLINDER 0.5150 DAN $(14)=0.50536$

RES $=15$ CYLINDER 0.5150 DAN(15) $=0.63264$

RES $=23$ CYLINDER 0.5150 DAN $(23)=0.46920$
RES $=24$ CYLINDER 0.5150 DAN $(24)=0.63217$ 
RES $=25$ CYLINDER 0.5150 DAN (25) $=0.79514$
RES $=26$ CYLINDER 0.5150 DAN 26$)=0.97782$ END MORE

BAW-1645-4, EXPIMT-2464 CASE C-2-C, DANCOFF FACTOR FOR EACH FUEL PIN READ PARAM TME $=40$ FDN $=Y E S$ NUB $=$ YES END PARAM

READ GEOM

UNIT 1

ZHEMICYL-Y $210.6030142 .5400 \quad-0.3180$

ZHEMICYL-Y $2 \quad 1 \quad 0.6030 \quad 156.1220 \quad 142.5400$

UNIT 3

ZHEMICYL+Y $210.6030142 .5400 \quad-0.3180$

UNIT 4

ZHEMICYL+Y $210.6030156 .1220 \quad 142.5400$

UNIT 5

2HEMICYL-Y $13 \quad 1 \quad 0.5150142 .5400 \quad 0.0$

$\begin{array}{llllll}\text { ZHEMICYL-Y } & 0 & 1 & 0.5220 & 142.5400 & 0.0 \\ \text { ZHEMICYL-Y } & 1 & 1 & 0.6030 & 142.5400 & -0.3180\end{array}$

UNIY 6

UNIT 6 -

ZHEMICYL-Y 23 i 0.5150153 .3400142 .5400

$\begin{array}{llllll}\text { ZHEMICYL-Y } & 0 & 1 & 0.5220 & 155.8040 & 142.5400 \\ \text { ZHEMICYL-Y } & 1 & 1 & 0.6030 & 156.1220 & 142.5400\end{array}$

UNIT 7

ZHEMICYL-Y $14 \quad 1 \quad 0.5150142 .5400 \quad 0.0$

$\begin{array}{llllll}\text { ZHEMICYL-Y } & 0 & 1 & 0.5220 & 142.5400 & 0.0 \\ \text { ZHEMICYL-Y } & 1 & 1 & 0.6030 & 142.5400 & -0.3180\end{array}$

UNIT 8

ZHEMICYL-Y $24 \quad 1 \quad 0.5150153 .3400 \quad 142.5400$

ZHEMICYL-Y O $10.5220155 .8040 \quad 142.5400$

ZHEMICYL-Y 110.6030156 .1220

UNIT 9

142.5400

ZHEMICYL-Y $16 \quad 1 \quad 0.5150 \quad 142.5400 \quad 0.0$

ZHEMICYLY

$\begin{array}{llllll}\text { 2HEMICYL-Y } & 1 & 1 & 0.6030 & 142.5400 & -0.3180\end{array}$

UNIT 10

ZHEMICYL-Y $26 \quad 1 \quad 0.5150153 .3400 \quad 142.5400$

$\begin{array}{llllll}\text { ZHEMICYL-Y } & 0 & 1 & 0.5220 & 155.8040 & 142.5400 \\ \text { ZHEMICYL-Y } & 1 & 1 & 0.6030 & 156.1220 & 142.5400\end{array}$

UNIT 11

ZHEMICYL+Y $13,0.5150142 .5400 \quad 0.0$

ZHEMICYL+Y 010.5520142 .540000 .00

ZHEMICYL+Y 12 1 0.6030 142.5400 -0.3180

2HEMICYL+Y $23 \quad 1 \quad 0.5150 \quad 153.3400 \quad 142.5400$

ZHEMICYL+Y $010.5220155 .8040 \quad 142.5400$

ZHEMICYL+Y 110.6030156 .1220142 .5400

UNIT 13

ZHEMICYL+Y $14 \quad 1 \quad 0.5150 \quad 142.5400 \quad 0.0$

$\begin{array}{llllll}\text { ZHEMICYL+Y } & 0 & 1 & 0.5220 & 142.5400 & 0.0 \\ \text { ZHEMICYL+Y } & 1 & 1 & 0.6030 & 142.5400 & -0.3180\end{array}$

2HEMICYL+Y $1110.6030142 .5400-0.3180$

UNIT 14

$\begin{array}{lrllll}\text { ZHEMICYL+Y } 24 & 1 & 0.5150 & 153.3400 & 142.5400 \\ \text { ZHEMICYL+Y } & 0 & 0.5220 & 155.8040 & 142.5400\end{array}$ ZHEMICYL+Y 110.6030156 .1220142 .5400 UNIT 15

ZHEMICYL+Y $16 \quad 1 \quad 0.5150 \quad 142.5400 \quad 0.0$

ZHEMICYL +Y 010.5220142 .54000 .0

ZHEMICYL+Y $110.6030142 .5400-0.3180$

UNIT 16

ZHEMICYL+Y $26,0.5150 \quad 153.3400 \quad 142.5400$ ZHEMICYL+Y 10.5220155 .8040142 .5400 ZHEMICYL+Y 110.6030156 .1220142 .5400

UNIT 17

ZHEMICYL-X $14 \quad 1 \quad 0.5150142 .5400 \quad 0.0$

$\begin{array}{llllll}\text { ZHEMICYLL-X } & 0 & 1 & 0.5220 & 142.5400 & 0.0 \\ \text { ZHEMICYYL-X } & 1 & 1 & 0.6030 & 142.5400 & -0.3180\end{array}$

UNIT 18

ZHEMICYL-X $24 \quad 1 \quad 0.5150 \quad 153.3400 \quad 142.5400$

2 HEMICYL-X O 10.5220155 .8040142 .5400

ZHEMICYL-X 110.6030156 .1220142 .5400

UNIT 19

ZHEMICYL-X $15 \quad 1 \quad 0.5150 \quad 142.5400 \quad 0.0$

ZHEMICYL-X 0 I 0.5220142 .54000 .0

ZHEMICYL-X $1110.6030 \quad 142.5400-0.3180$

ZHEMT 20

ZHEMICYL-X $25 \quad 10.5150153 .3400 \quad 142.5400$

ZHEMICYL-X 0 1 0.5220155 .8040 142.5400

ZHEMICYL-X 110.6030156 .1220142 .5400 UNIT 21

ZHEMICYL-X $16 \quad 1 \quad 0.5150 \quad 142.5400 \quad 0.0$

2HEMICYL-X 010.5220142 .54000 .0

ZHEMICYL-X $110.6030142 .5400-0.3180$

UNIT 22

ZHEMICYL-X $26 \quad 1 \quad 0.5150 \quad 153.3400 \quad 142.5400$ 


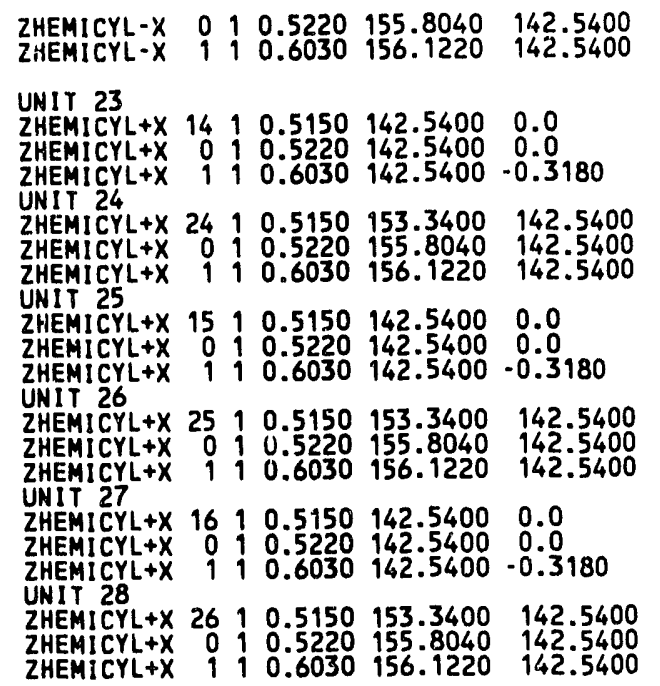

UNIT 29

COM= 'CUBOID BUILT AROUND UNIT 1 '

ZHEMICYL-Y $210.6030 \quad 142.5400-0.3180$

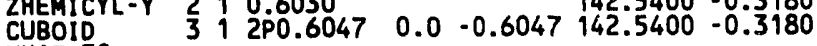

UNIT 30 COMOID BUILT AROUND UNIT 2

ZHEMICYL-Y $210.6030 \quad 156.1220142 .5400$ $\begin{array}{llllllll}\text { CUEMIDY } & 0 & 1 & 0.6050 .6047 & 0.0 & -0.6047 & 156.1220 & 142.5400\end{array}$ UNIT 31

COM=I CUBOID BUILT AROUND UNIT 3'

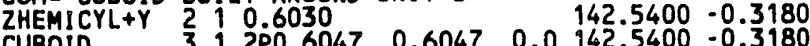

CUBOID

UNIT 32 BOID BUILT AROUND UNIT 41

COM='CUBOID BUILT AROUND UNIT 4'

$\begin{array}{lllllllll}\text { ZHEMICYL+Y } & 2 & 1 & 0.6030 & & & 156.1220 & 142.5400 \\ \text { CUBOID } & 0 & 1 & 2 \mathrm{P} 0.6047 & 0.6047 & 0.0 & 156.1220 & 142.5400\end{array}$

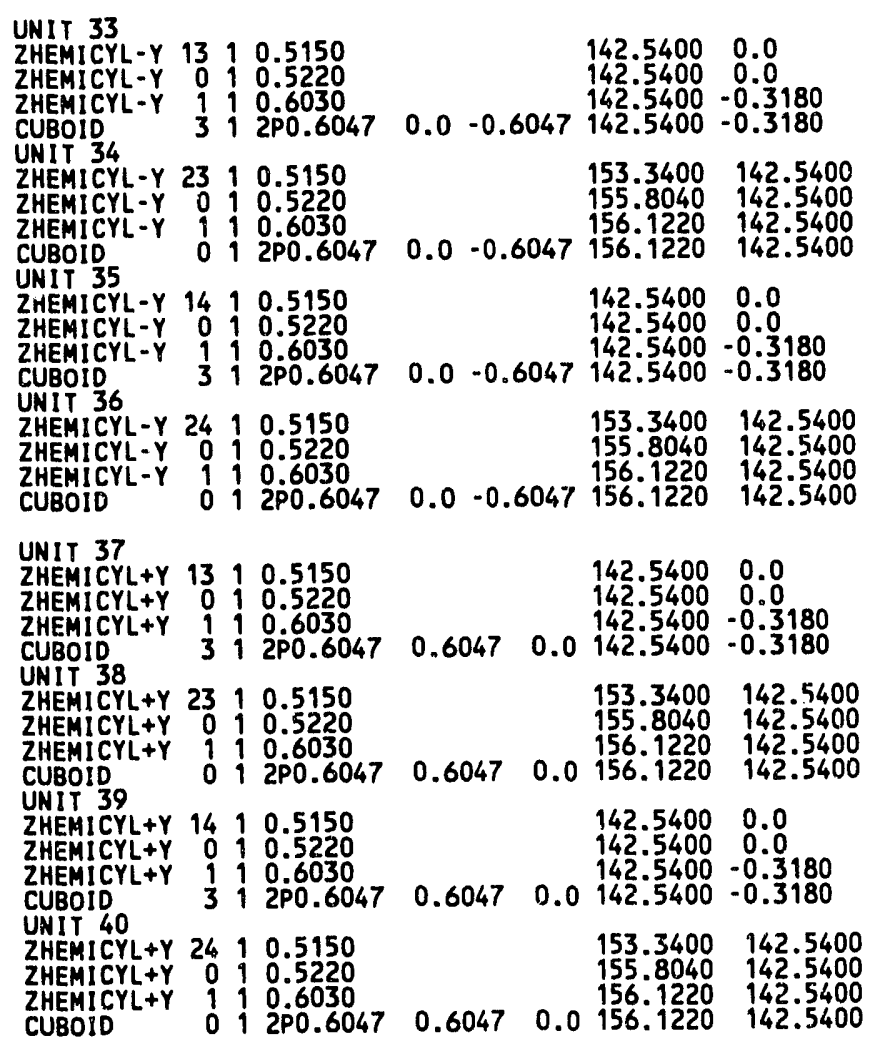


UNIT 41

UNIT 41 COMBID BUILT AROUND UNIT 3 HITH UNITS $5 \& 17$ ADDED AS HOLES' COM=ICUBOID BUILT AROUND UNIT 3 HITH UNITS 5 \& IT ADDED AS HOLES

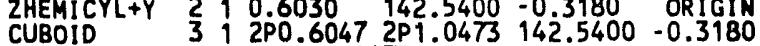
$\begin{array}{lrlll}\text { HOLE } & 5 & 0.001 .0473 & 0.0 \\ \text { HOLE } & 17 & 0.6047 & 0.0 & 0.0\end{array}$

UNIT 42

COM=, CUBOID BUILT AROUND UNIT 4 WITH UNITS $6 \& 18$ ADDED AS HOLES' ZHEMICYLTY $2,0.6030 \quad 156.1220142 .5400$ ORIGIN $0.0-1.0473$ CUBOID 012 PO.6047 2P1.0473 156.1220 142.5400

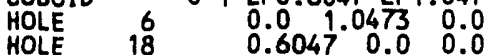

UNIT 43

COM=' CUBOID BUILT AROUND UNIT 11 WITH UNITS $921 \& 23$ ADDED AS HOLES' ZHEMICYL+Y 1310.5150142 .54000 .0000 ORIGIN $0.0-1.0473$

ZHEMICYLYY $010.5220 \quad 142.5400 \quad 0.0000$ ORIGIN $0.0-1.0473$

ZHEMICYL+Y $1110.6030 \quad 142.5400-0.3180$ ORIGIN $0.0-1.0473$

CUBOID $312 P 0.60472 P 1.0473142 .5400-0.3180$

HOLE 990.01 .04730 .0

$\begin{array}{lllll}\text { HOLE } & 21 & 0.6047 & 0.0 & 0.0 \\ \text { HOLE } & 23 & -0.6047 & 0.0 & 0.0\end{array}$

UNIT 44

CUBOID BUILT AROUND UNIT 12 WITH UNITS $1022 \& 24$ ADDED AS HOLES'

ZHEMICYL+Y $2310.5150 \quad 153.3400142 .5400$ ORIGIN $0.0-1.0473$

ZHEMICYLY $010.5220 \quad 155.8040142 .5400$ ORIGIN $0.0-1.0473$

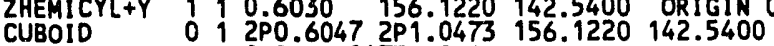

HOLE $10 \quad 0.01 .04730 .0$

$\begin{array}{lllll}\text { HOLE } & 22 & 0.6047 & 0.0 & 0.0 \\ \text { HOLE } & 24 & -0.6047 & 0.0 & 0.0\end{array}$

UNIT 45

COM='CUBOID BUILT AROUND UNIT 13 WITH UNITS $921 \& 27$ AUDED AS HOLES'

2HEMICYL+Y $14: 0.5150 \quad 142.5400 \quad 0.0000$ ORIGIN $0.0-1.0475$

ZHEMICYL+Y $010.5220 \quad 142.54000 .0000$ ORIGIN $0.0-1.0473$

CUBOID $312 \mathrm{PP0} .60472 \mathrm{P} 1.0473142 .5400-0.3180$

HOLE 9 O 0.091 .04730 .0

$\begin{array}{lllll}\text { HOLE } & 21 & 0.6047 & 0.0 & 0.0 \\ \text { HOLE } & 27 & -0.6047 & 0.0 & 0.0\end{array}$

UNIT 46

COM=' CUBOID BUILT AROUND UNIT 14 WITH UNITS $1022 \& 28$ ADDED AS HOLES

ZHEMICYL+Y 24 i $0.5150 \quad 153.3400142 .5400$ ORIGIN $0.0-1.0473$

ZHEMICYL+Y $010.5220 \quad 155.8040142 .5400$ ORIGIN $0.0-1.0473$

ZHEMICYL+Y 110.6030 156.1220 142.5400 ORIGIN $0.0-1.0473$

CUBOID $012 \mathrm{PPO} 0.60472 \mathrm{2P} 1.0473 \quad 156.1220142 .5400$

HOLE $10 \quad 0.011 .04730 .0$

$\begin{array}{lllll}\text { HOLE } & 22 & 0.6047 & 0.0 & 0.0 \\ \text { HOLE } & 28 & -0.6047 & 0.0 & 0.0\end{array}$

UNIT 47

COM $=$ ' CUBOID BUILT AROUND UNIT 11 WITH UNITS $917 \& 27$ ADDED AS HOLES

ZHEMICYL+Y $1310.5150 \quad 142.5400 \quad 0.0000$ ORIGIN $0.0-1.0473$

ZHEMICYLIY $0010.5220 \quad 142.5400 \quad 0.0000$ ORIGIN $0.0-1.0475$

ZHEMICYITY $110.6030 \quad 142.5400-0.3180$ ORIGIN $0.0-1.0473$

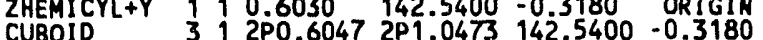

HOLE 9 S 0.091 .04730 .0

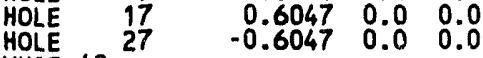

UNIT 48

COM= CUBOID BUILT AROUND UNIT 12 WITH UNITS $1018 \& 28$ ADDED AS HOLES ZHEMICYL+Y $2310.5150 \quad 153.3400142 .5400$ ORIGIN $0.0-1.0473$

ZHEMICYL+Y $010.5220 \quad 155.8040142 .5400$ ORIGIN $0.0-1.0473$

ZHEMICYL+Y 110.6030 156.1220 142.5400 ORIGIN 0.0

CUBOID $012 P 0.60472 P 1.0473156 .1220142 .5400$

HOLE $10 \quad 0.01 .04730 .0$

HOLE $18 \quad 0.60470 .00 .0$

UNIT 49

COM=' CUBOID BUILT AROUND UNIT 3 WITH UNITS 5 \& 23 ADDED AS HOLES

ZHEMICYL+Y 2 : $0.6030 \quad 142.5400-0.3180$ ORIGIN $0.0-1.0473$

CUBOID $312 P 0.60472 P 1.0473142 .5400-0.3180$

$\begin{array}{lllll}\text { HOLE } & 5 & 0.0 & 1.0473 & 0.0 \\ \text { HOLE } & 23 & -0.6047 & 0.0 & 0.0\end{array}$

COM=ICUBOID BUILT AROUND UMIT 4 WITH UNITS $6 \& 24$ ADDED AS HOLES

ZMEMICYLYY $210.6030 \quad 156.1220142 .5400$ ORIGIN $0.0-1.0473$

CUBOID O 12 P0.6047 2P1.0473 156.1220 142.5400

HOLE 60.01 .04730 .0

UNIT 51 COH=I CUBOID BUILT AROUND UNIT 11 HITH UNITS 5 \& 19 ADDED AS HOLES'

CONA=' CUBOID BUILT AROUND UNIT 11 HITH UNITS 5 \& 19 ADDED AS HOLES

$\begin{array}{lrllllll}\text { ZHEMICYL+Y } & 13 & 1 & 0.5150 & 142.5400 & 0.0 & \text { ORIGIN } & 0.0-1.0473 \\ \text { ZHEMICYL+Y } & 0 & 1 & 0.5220 & 142.5400 & 0.0 & \text { ORIGIN } & 0.0-1.0473\end{array}$ 


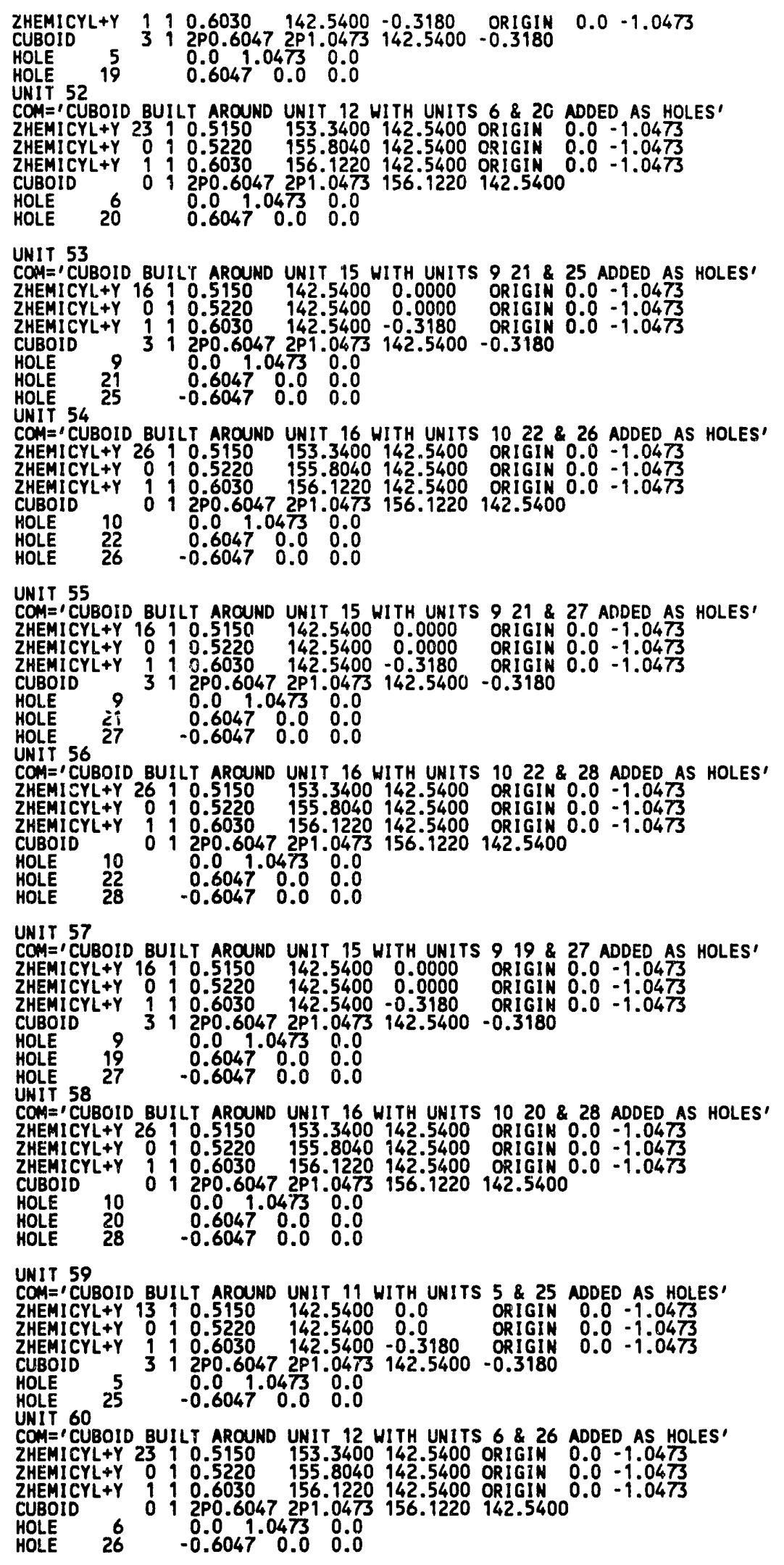


UNIT 61

COM='CUBOID BUILT AROUND UNIT 1 WITH UNITS $11 \& 17$ ADDED AS HOLES'

ZHEMICYL-Y $210.6030142 .5400-0.3180$ ORIGIN 0.01 .0473

CUBOID 312 P $0.60472 P 1.0473142 .5400-0.3180$

$\begin{array}{lllll}\text { HOLE } & 11 & 0.0-1.0473 & 0.0 \\ \text { HOLE } & 17 & 0.6047 & 0.0 & 0.0\end{array}$

HOLE 62

COM='CUBOID BUILT AROUND UNIT 2 WITH UNITS 12 \& 18 ADDED AS HOLES'

ZHEMICYL-Y 210.6030 156.1220 942.5400 ORIGIN 0.01 .0473

CUBOID 012 P0.6047 2P1.0473 156.1220142 .5400

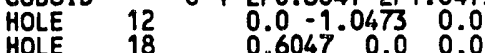

UNIT 63

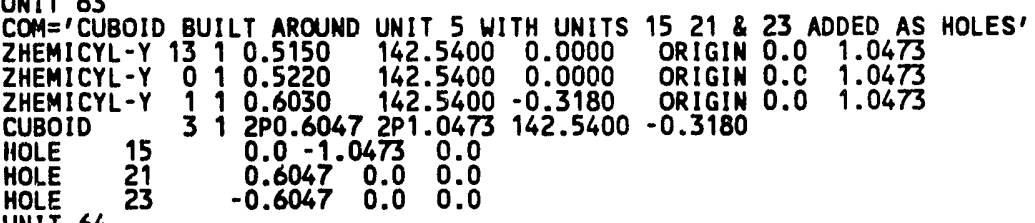

COM=ICUBOID BUILT AROUND UNIT 6 HITH UNITS $1622 \& 24$ ADDED AS HOLES'

ZHEMICYL-Y 2310.5150 T53.3400 142.5400 ORIGIN 0.019 .0475

ZHEMICYL-Y $010.5220 \quad 155.8040142 .5400$ ORIGIN 0.019 .0473

ZHEMICYL-Y 110.6030 156.1220 142.5400 ORIGIN 0.0190473

CUBOID $\quad 012 P 0.60472 P 1.0473156 .1220142 .5400$

HOLE $16 \quad 0.0-1.0473 \quad 0.0$

$\begin{array}{lllll}\text { HOLE } & 22 & 0.6047 & 0.0 & 0.0 \\ \text { HOLE } & 24 & -0.6047 & 0.0 & 0.0\end{array}$

UNIT 65

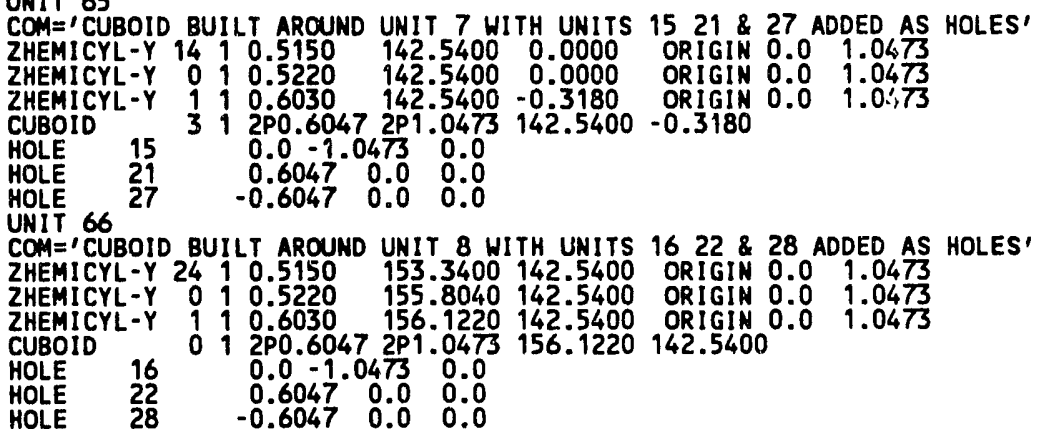

UNIT 67

COM $=1$ CUBOID BUILT AROUND UNIT 5 WITH UNITS $1517 \& 27$ ADDED AS HOLES'

ZHEMICYL-Y 13130.5150 142.5400 0.0000 ORIGIN 0.019 .0473

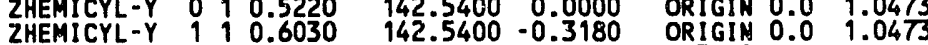

$\begin{array}{lllllll}\text { ZHEMICYL-Y } & 1 & 1 & 0.6030 & 142.5400 & -0.3180 & \text { ORIGIN } \\ \text { CUBOID } & 3 & 1 & 2 \mathrm{P} 0.6047 & 2 \mathrm{P} 1.0473 & 142.5400 & -0.3180\end{array}$

HOLE 15 O.0-1.0473 0.0

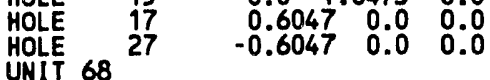

COM $=$ ' CUBOID BUILT AROUND UNIT 6 HITH UNITS $1618 \& 28$ ADDED AS HOLES'

ZHEMICYL-Y $2310.5150 \quad 153.3400142 .5400$ ORIGIN 0.01 .0473

ZHEMICYLY $010.5220 \quad 155.8040142 .5400$ ORIGIN 0.01 .0473

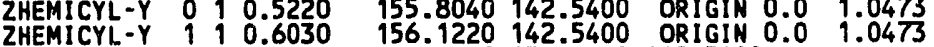

$\begin{array}{lllllll}\text { ZHEMICYL-Y } & 1 & 1 & 0.6030 & 156.1220 & 142.5400 & \text { ORIGIN } \\ \text { CUBOID } & 0 & 1 & 2 \mathrm{PO} 0.6047 & 2 \mathrm{P} 1.0473 & 156.1220 & 142.5400\end{array}$

HOLE 16 O.0 -1.04730 .0$

$\begin{array}{lllll}\text { HOLE } & 18 & 0.6047^{\circ} & 0.0 & 0.0 \\ \text { HOLE } & 28 & -0.6047 & 0.0 & 0.0\end{array}$

UNIT 69

COM=' CUBOID BUILT AROUND UNIT 1 WITH UNITS $11 \& 23$ ADDED AS HOLES'

ZHEMICYL-Y $210.6030 \quad 142.5400-0.3180$ ORIGIN 0.01 .0473

CUBOID 3 ; 2 P0.6047 2P1.0473 $142.5400-0.3180$

HOLE 11 (1 $0.0-1.04730 .0$

HOLE $23 \quad-0.6047 \quad 0.0 \quad 0.0$

COM=' CUBOID BUILT AROUND UNIT 2 WITH UNITS $12 \& 24$ ADDED AS HOLES'

ZHEMICYL-Y 210.6030 156.1220 142.5400 ORIGIN 0.01 .0473

CUBOID 012 2P0.6047 2P1.0473 156.1220142 .5400

HOLE $\quad 12 \quad 0.0-1.04730 .0$

UNIT 71

COM='FUEL MODULE W/O AL SIDE PLATES, BELOW WATER'

ARRAY $1-9.0705-8.9832-0.3180$

CUBOID 3 i 2 P9.5250 2 P9.5250 $142.5400-0.3180$ 
CUBOID 412 2P9.5250 2P9.5250 $142.5400-1.3848$

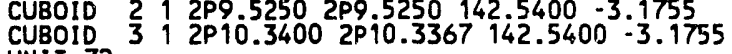

UNIT 72

COM $=$ ' $F U E L$ MODULE H/O AL SIDE PLATES, ABOVE HATER

COMA $=$ FUEL MOULE H/OAL SIDE PLATES
ARRAY $2-9.0705-8.9832142 .5400$

CUBOID O I $2 P 9.52502 P 9.5250156 .1220142 .5400$

CUBOID 2 I 12 PP.5250 2P9.5250 157.7095142 .5400

CUBOID 0 i 2 P 10.34002 P 10.3367157 .7095142 .5400

UNIT 73

COM='FUEL MOOULE WITH AL SIDE PLATES, BELOW HATER'

ARRAY $1-9.0705-8.9832-0.3180$

CUBOID 3 i 2 P9. $07052 P 9.3957142 .5400-0.3180$

CUBOID 21 2P9.0705 2P9.5250 $142.5400-0.3180$

CUBOID 211 2P9..0705 2P9.5250 $142.5400-0.3180$

CUBOID 4 i 2 PP9.5250 2P9.5250 $142.5400-1.3848$

CUBOID $292 P 9.52502 P 9.5250142 .5400-3.1755$

CUBOID $312 P 10.34002 P 10.3367142 .5400: 3.1755$

UNIT 74

COM='FUEL MOOULE WITH AL SIDE PLATES, ABOVE WATER'

ARRAY $2-9.0705-8.9832$ 142.5400

CUBOID 0 i $2 P 9.07052$ PP9.3957 156.1220942 .5400

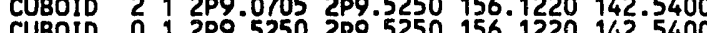

CUBOID 21 2P9.55250 2P9.5250 157.7095142 .5400

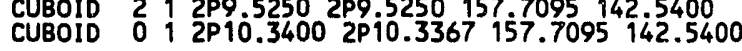

UNIT 75

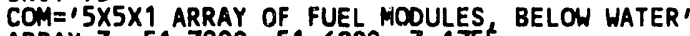

ARRAY $3-51.7000-51.6880-3.1755$

CUBOID 31 2P60.9600 2P60.9600 $142.5400-3.1755$

CUBOID 212 P60.9600 2 P60.9600 $142.5400-118115$

CYLINDER 3 1 $137.1600142 .5400-14.6815$

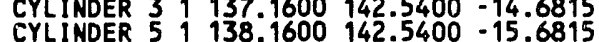

CUBOID 014 P $138.1600142 .5400^{-30.9215}$

UNIT 76

COM $=15 \times 5 \times 1$ ARRAY OF FUEL MOOULES ABOVE WATER'
ARRAY $4-51.7000-51.6880$
C 142.5400

CUBOID ${ }^{\circ} i 2 P 60.96002 P 60.9600 \quad 157.7095142 .5400$

CYLINDER $01137.1600 \quad 157.7095142 .5400$

CYLINDER 51138.1600157 .7095142 .5400

CUBOID 014 P 138.1600157 .7095142 .5400

GLOBAL

UNIT 77

COM $=$ ' STACK UNIT 76 ATOP UNIT 75

ARRAY $5-138.1600-138.1600-30.9215$

END GEOH

READ ARRAY

$A R A=1 \quad$ NUX $=15$ NUY $=10$ NUZ $=1$

COM $=$ 'FUEL MODULE ARRAY, BELOW WATER'

FILL 2933 11R35 3329

$414311 R 454749$

5015

$616311 R 656769$

313711 R39 3731 END FILL

ARA $=2$ NUX $=15$ NUY $=10$ MUZ $=1$

COM='FUEL MODULE ARRAY, ABOVE WATER'

FILL $303411 R 363430$

$424411 R 464850$

5254 11R56 $5860 \quad 5015$

$626411 R 666870$

$323811 R 403832$ END FILL

ARA $=3$ NUX $=5$ NUY $=5$ NUZ $=1$

COM $=$ 'ARRAY OF FUEL MOOULES BELOW WATER'

FILL

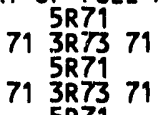

71 3R73 71 END FILL

$A R A=4$ NUX $=5$ NUY $=5$ NUZ $=1$

ARA $=4$ ARRAY OF FUEL MODULES ABOVE WATER'

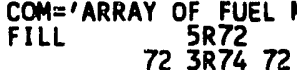

72 SRT4 72

72 SRT4 72

$A R A=5$ NUX $=1$ NUY $=1$ MUZ $=2$

COM='STACKING ABOVE WATER UNIT ATOP BELOW WATER UNIT'

FILL 7576 END FILL

END ARRAY

READ MIXT EPS=1. END MIXT 
READ BOUNDS - $2 B=$ =CONCB END BOUNDS

READ START NST $=1$ END START

END DATA

END

VALIDATIO'N CASE (CLTO4)
=CSAS25

BAH-1645-4, EXP'MT-2472, CASE C-2-C, DANCOFF FACTOR FOR EACH FUEL PIN 27GROUPNDF4 LATTICECELL

AL $11.0 \quad 291.5$ END

AL $21.0 \quad 291.5$ END

A

B2RON $34.7646-5291.5$ END

H2O 4 $0.75-291.5$ END

BORON $43.5735-5291.5$ END

AL 4 CARBONSTEEL 51.0291 .5 END

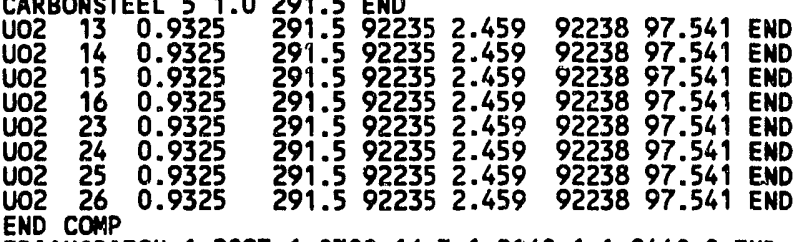

TRIANGPITCH 1.20931 .03001631 .206011 .04400 END

MORE DATA RES $=13$ CYLINDER 0.5150 DAN(13) $=0.37813$

RES $=14$ CYLINDER 0.5150 DAN 14$)=0.50543$

RES $=15$ CYLINDER 0.5150 DAN( 15) $=0.63273$

RES $=23$ CYLINDER $0.5150 \operatorname{DAN}(23)=0.46920$

RES $=24$ CYLIMDER 0.5150 DAN(24) $=0.63217$

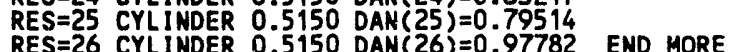

8AW-1645-4, EXP'MT-2472 CASE C-2-C, DANCOFF FACTOR FOR EACH FUEL PIN READ PARAM TME $=40$ FDN=YES NUB=YES END PARAM

READ GEOM

UNIT 1

ZHEMICYL-Y $2 \quad 1 \quad 0.6030145 .6400 \quad-0.3180$

UNIT 2

2HEMICYL-Y 210.6030156 .1220145 .6400

UNIT

ZHEMICYL+Y $210.6030145 .6400-0.3180$

ZHEMICYL+Y $210.6030156 .1220 \quad 145.6400$

UNIT 5

ZHEMICYL-Y $1313 \quad 0.5150 \quad 145.6400 \quad 0.0$

ZHEMICYL-Y 0 1 $10.5220145 .6400 \quad 0.018$

UNIT 6

ZHEMICYL-Y $23 \quad 10.5150153 .3400 \quad 145.6400$

ZHEMICYLY 2310.5520155 .3400145 .6400

ZHEMICYL-Y $190.6030 \quad 156.1220145 .6400$

ZHEMICY

ZHEMICYL-Y $\begin{array}{lllllll}14 & 1 & 0.5150 & 145.6400 & 0.0\end{array}$

ZHEMICYL-Y O $90.5220145 .6400 \quad 0.0$

ZHEMICYL-Y $110.6030145 .6400-0.3180$

UNIT 8

ZHEMICYL-Y $24 \quad 1 \quad 0.5150153 .3400 \quad 145.6400$

ZHEMICYL-Y O $10.5220155 .8040 \quad 145.6400$

ZHEMICYL-Y 190.6030 156.1220 145.6400

2HENTCYL

ZHEMICYL-Y $\begin{array}{llllll}16 & 1 & 0.5150 & 145.6400 & 0.0\end{array}$

ZHEMICYL-Y $0110.5220145 .6400 \quad 0.0$

ZHEMICYL-Y $110.6030145 .6400-0.3180$ UNIT 10

ZHEMICYL-Y $26110.5150 \quad 153.3400 \quad 145.6400$

ZHEMICYL-Y $0110.5220 \quad 155.8040 \quad 145.6400$

ZHEMICYL-Y 190.6030156 .1220145 .6400

UNIT 11

ZHEMICYL+Y $13130.5150 \quad 145.6400 \quad 0.0$

ZHEMICYL+Y $010.5220145 .6400 \quad 0.0$

ZHEMICYL+Y $1110.6030145 .6400-0.3180$ UNIT 12

ZHEMICYL+Y $23 \quad 1 \quad 0.5150153 .3400 \quad 145.6400$

ZHEMICYL+Y $010.5220 \quad 155.8040 \quad 145.6400$

ZHEMICYL+Y 110.6030156 .1220145 .6400

UNIT 13

ZHEMICYL +Y $1410.5150145 .6400 \quad 0.0$

ZHEMICYL+Y $0190.5220145 .6400 \quad 0.0$

$\begin{array}{llllll}\text { ZHEMICCYL+Y } & 0 & 1 & 0.5220 & 145.6400 & 0.0 \\ \text { ZHEMICYLLY } & 1 & 1 & 0.6630 & 145.6400 & -0.3180\end{array}$

UNIT 14

ZHEMICYL+Y $24,0.5150153 .3400 \quad 145.6400$

ZHEMICYL+Y 0 i $0.5220155 .8040 \quad 145.640$ 


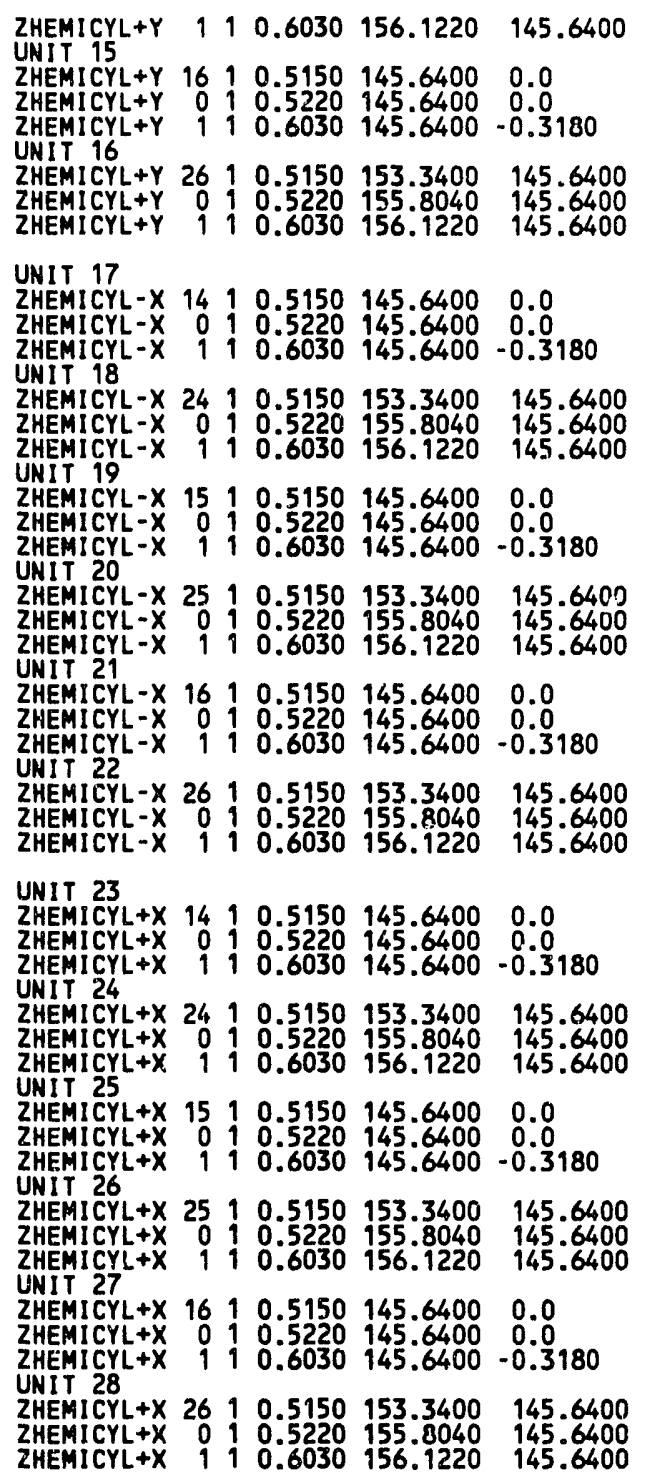

ZHEMICYL+X 110.6030156 .1220145 .6400

UNIT 29

ZHEMICYL-Y 2 T $0.6030 \quad 145.6400-0.3180$

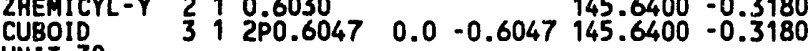
UNIT 30

COM='CUBOID BUILT AROUND UNIT 2 '

ZHEMICYL-Y 2 I $0.6030 \quad 156.1220145 .6400$ $\begin{array}{llllllll}\text { CUBOID } & 0 & 1 & 2 \mathrm{P} 0.6047 & 0.0 & -0.6047 & 156.1220 & 145.6400\end{array}$ UNIT 31

COM $=$ 'CUBOID BUILT AROUND UNIT 3 '

ZHEMICYL $+Y 210.6030$

$\begin{array}{lllllll}2 & 2 & 0.6030 & 0.620 & 145.6400 & -0.3180\end{array}$

UNIT 32

COM=ICUBOID BUILT AROUND UNIT 4'

ZHEMICYL+Y $210.6030 \quad 156.1220145 .6400$ $\begin{array}{llllllll}\text { ZHEMICYL+Y } & 2 & 1 & 0.6030 & & 156.1220 & 145.6400 \\ \text { CUBOID } & 0 & 1 & 2 \mathrm{P} 0.6047 & 0.6047 & 0.0 & 156.1220 & 145.6400\end{array}$

UNIT 33

\begin{tabular}{|c|c|c|c|c|}
\hline $\begin{array}{l}\text { ZHEMICYL-Y } \\
\text { ZHEMICYL-Y } \\
\text { ZHEMICYL-Y } \\
\text { CUBOOID }\end{array}$ & $\begin{array}{rll}13 & 1 & 0.5150 \\
0 & 1 & 0.5220 \\
1 & 1 & 0.6030 \\
3 & 1 & 2 p 0.6047\end{array}$ & $0.0-0.6047$ & $\begin{array}{l}145.6400 \\
145.6400 \\
145.6400 \\
145.6400\end{array}$ & $\begin{array}{l}0.0 \\
0.0 \\
-0.3180 \\
-0.3180\end{array}$ \\
\hline $\begin{array}{l}\text { HEMICYL-Y } \\
\text { HEMICYL-Y } \\
\text { HEMICYL-Y }\end{array}$ & $\begin{array}{rll}23 & 1 & 0.5150 \\
0 & 1 & 0.5220 \\
1 & 1 & 0.6030 \\
0 & 1 & 2 \mathrm{p} 0.6047\end{array}$ & $0.0-0.6047$ & $\begin{array}{l}153.3400 \\
155.8040 \\
156.1220 \\
156.1220\end{array}$ & $\begin{array}{l}145.6400 \\
145.6400 \\
145.6400 \\
145.6400\end{array}$ \\
\hline
\end{tabular}




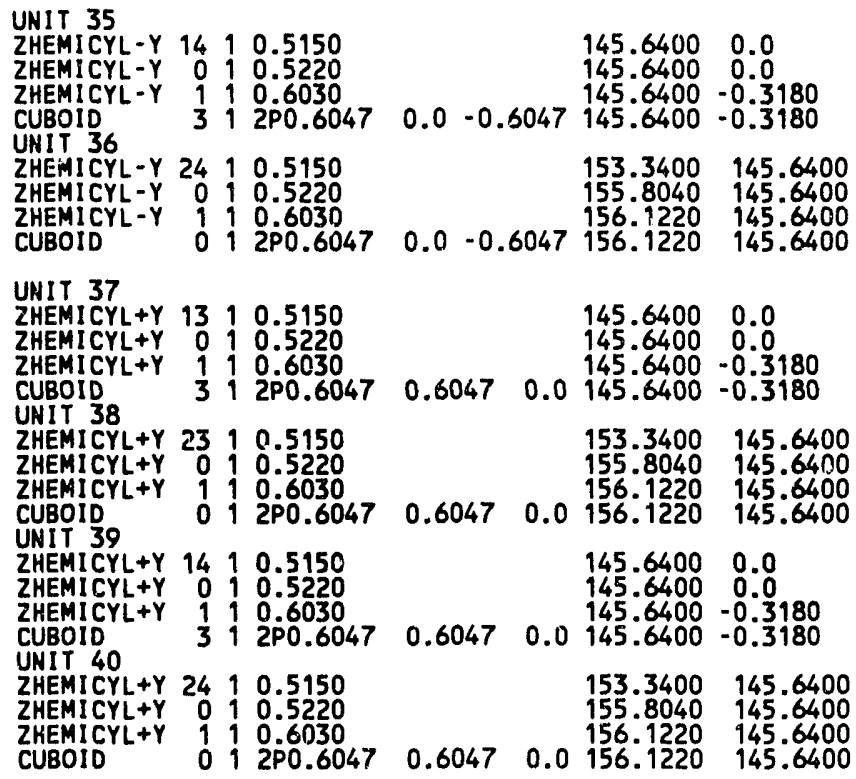

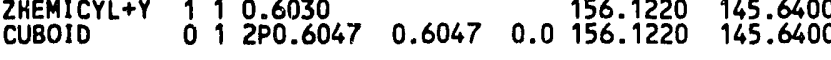

UNIT 41 COM=ICUBOID BUILT AROUND UNIT 3 WITH UNITS $5 \& 17$ ADDED AS HOLES

ZHEMICYL $+Y$ 2 10.6030 145.6400 -0.3180 ORIGIN $0.0-1.0473$

CUBOID 312 P0.6047 2P1.0473 $145.6400-0.3180$

$\begin{array}{lrlll}\text { HOLE } & 5 & 0.0 & 1.0473 & 0.0 \\ \text { HOLE } & 17 & 0.6047 & 0.0 & 0.0\end{array}$

UNIT $4217 \quad 0.60470 .0 \quad 0.0$

COM $=$ ' CUBOID BUILT AROUND UNIT 4 WITH UNITS $6 \& 18$ ADDED AS HOLES'

ZHEMICYL $+Y$ Y 10.6030156 .1220145 .6400 ORIGIN $0.0-1.0473$

ZHEMICYL+Y 2 T 0.60304756 .1220145 .6400 ORIGIN $0.0-1.0473$

$\begin{array}{lrrrrr}\text { CUBOID } & 0 & 1 & 2 P 0.6047 & 2 P 1.0473 \\ \text { HOLE } & 6 & 0.0 & 1.0473 & 0.0 \\ \text { HOLE } & 18 & 0.6047 & 0.0 & 0.0\end{array}$

UNIT 43

COMA='CUBOID BUILT AROUND UNIT 11 WITH UNITS 921 \& 23 ADDED AS HOLES'

ZHEMICYL+Y $1310.5150 \quad 145.6400 \quad 0.0000$ ORIGIN $0.0-1.0473$

ZHEMICYL+Y $010.5220 \quad 145.64000 .0000$ ORIGIN $0.0-1.0475$

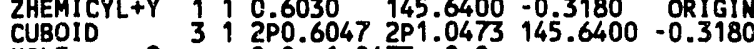

HOLE 9 P 0.01 .04730 .0

HOLE $21 \quad 0.60470 .00 .0$

HOLE 23

$\begin{array}{rll}-0.3047 & 0.0 & 0.0\end{array}$

UNIT 44

CHEMICYBOID BUILT AROUND UNIT 12 WITH UNITS $1022 \& 24$ ADDED AS HOLES

ZHEMICYL+Y $2310.5150 \quad 153.3400 \quad 145.6400$ ORIGIN $0.0-1.0473$

ZHEMICYL $+Y 110.6030156 .1220145 .6400$ ORIGIN $0.0-1.0473$

CUBOID 012 P0.6047 2 P1.0473 156.1220 145.6400

HOLE 10 O 0.01 .04730 .0

$\begin{array}{lllll}\text { HOLE } & 22 & 0.6047 & 0.0 & 0.0 \\ \text { HOLE } & 24 & -0.6047 & 0.0 & 0.0\end{array}$

UNIT 45

COM='CUBOID BUILT AROUND UNIT 13 WITH UNITS $921 \& 27$ ADDED AS HOLES'

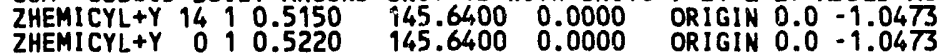

ZHEMICYL+Y $110.6030 \quad 145.6400-0.3180$ ORIGIN $0.0-1.0475$

CUBOID 3 i 2P0.6047 2P1.0473 $145.6400-0.3180$

HOLE 9 O 0.01904730 .0

$\begin{array}{lllll}\text { HOLE } & 21 & 0.6047 & 0.0 & 0.0 \\ \text { HOLE } & 27 & -0.6047 & 0.0 & 0.0\end{array}$

HOLE $4627 \quad-0.60470 .00 .0$

COM=1 CUBOID BUILT AROUND UNIT 14 HITH UNITS $1022 \& 28$ ADDED AS HOLES

ZHEMICYL +Y $24,0.5150$ 153.3400 145.6400 ORIGIN $0.0-1.0473$

ZHEMICYL +Y $10.5220 \quad 155.8040145 .6400$ ORIGIN $0.0-1.0475$

ZHEMICYL 1 Y 10.6030 156.1220 145.6400 ORIGIN $0.0-1.0473$

CUBOID $012 P 0.60472 P 1.0473156 .1220145 .6400$

HOLE 10 O $\quad 0.091 .04730 .0$

$\begin{array}{lllll}\text { HOLE } & 22 & 0.6047 & 0.0 & 0.0 \\ \text { HOLE } & 28 & -0.6047 & 0.0 & 0.0\end{array}$

UNIT 47

COM=ICUBOID BUILT AROUND UNIT 11 HITH UNITS $917 \& 27$ ADDED AS HOLES'

$\begin{array}{lllllll}\text { ZHEMICYLIY } & 13 & 1 & 0.5150 & 145.6400 & 0.0000 & \text { ORIGIN } 0.0-1.0473 \\ \text { ZHEMICYL+Y } & 0 & 1 & 0.5220 & 145.6400 & 0.0000 & \text { ORIGIN } 0.0-1.0473\end{array}$ 


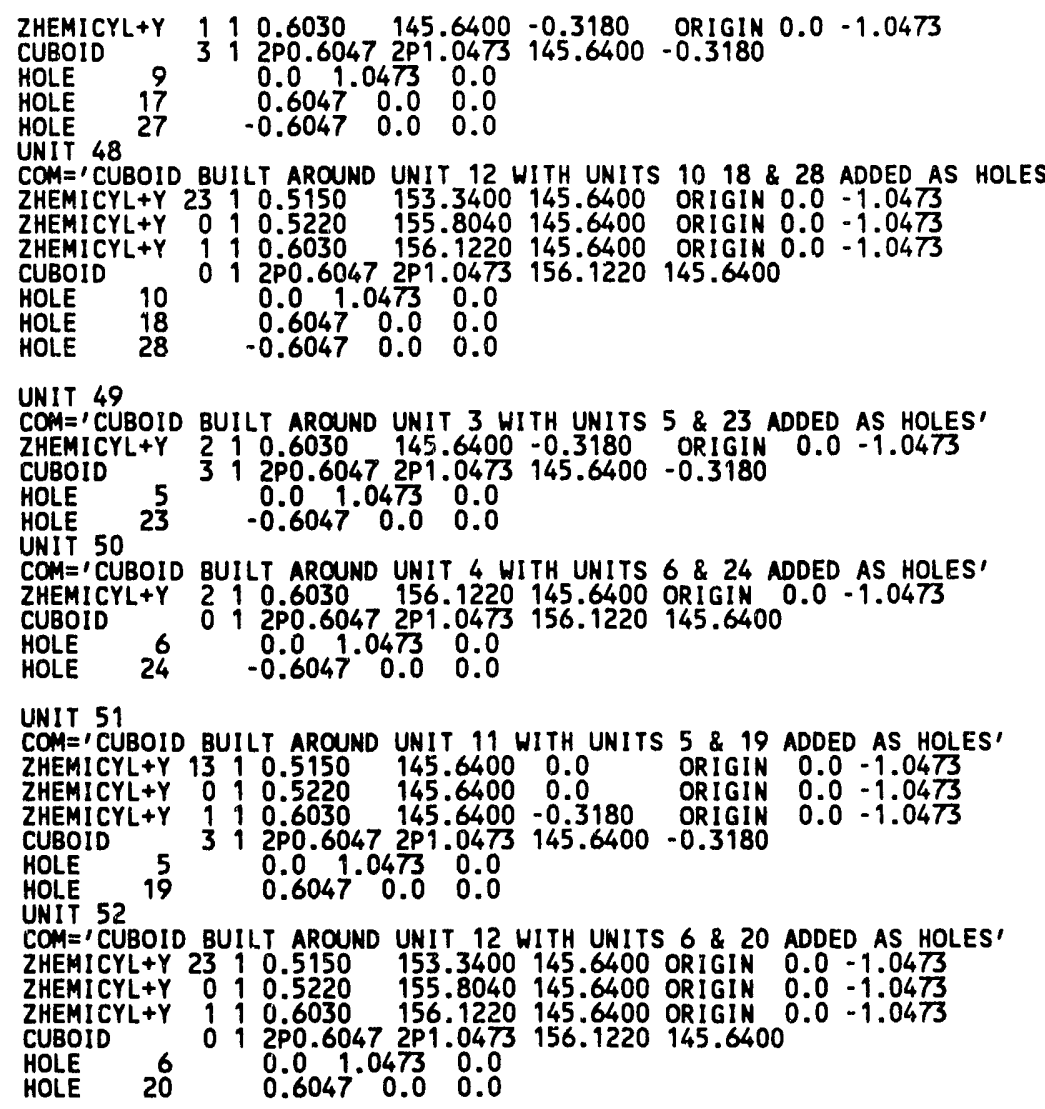

UNIT 53

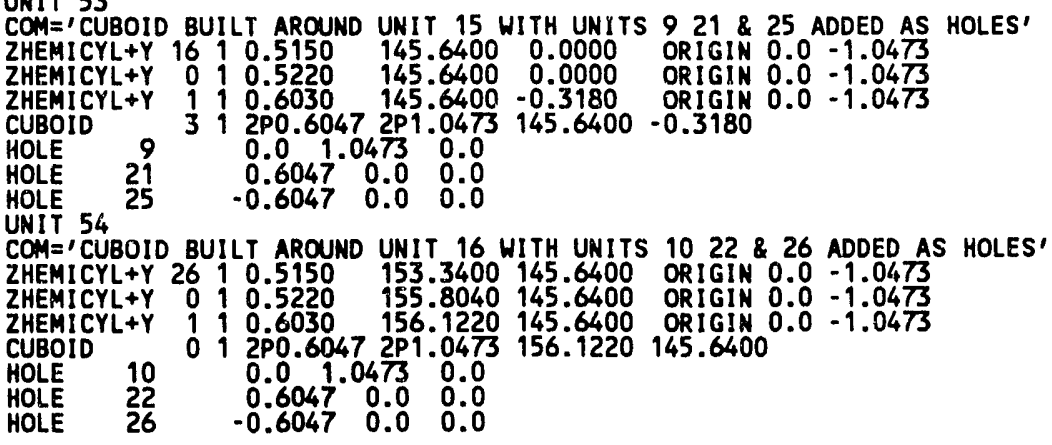

UNIT 55

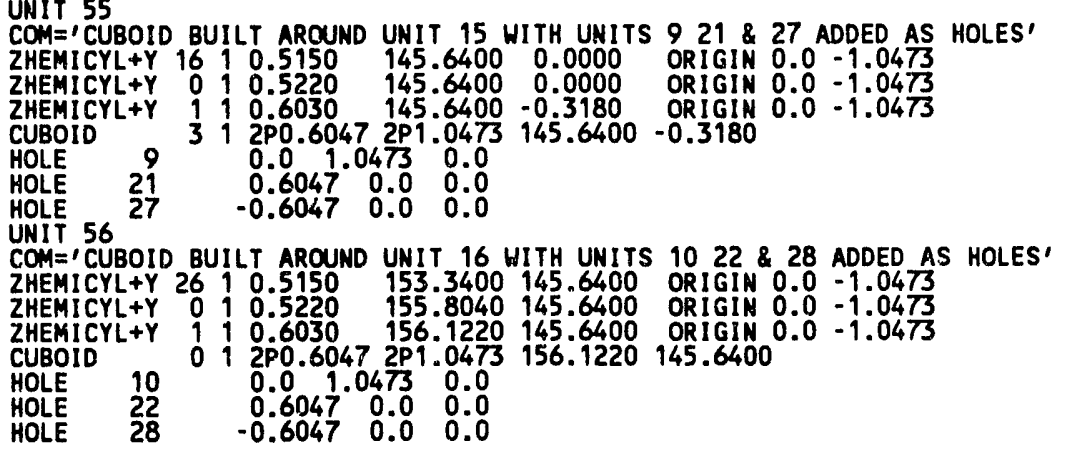

UNIT 57

COM='CUBOID BUILT AROUND UNIT 15 WITH UNITS $919 \& 27$ ADDED AS HOLES' ZHEMICYL+Y $16: \begin{array}{llllll}0.5150 & 145.6400 & 0.0000 & \text { ORIGIN } & 0.0 & -1.0473 \\ \text { ZHEMICYLTY } & 0 & 10.5220 & 145.6400 & 0.0000 & \text { ORIGIN } 0.0-1.0473\end{array}$ 


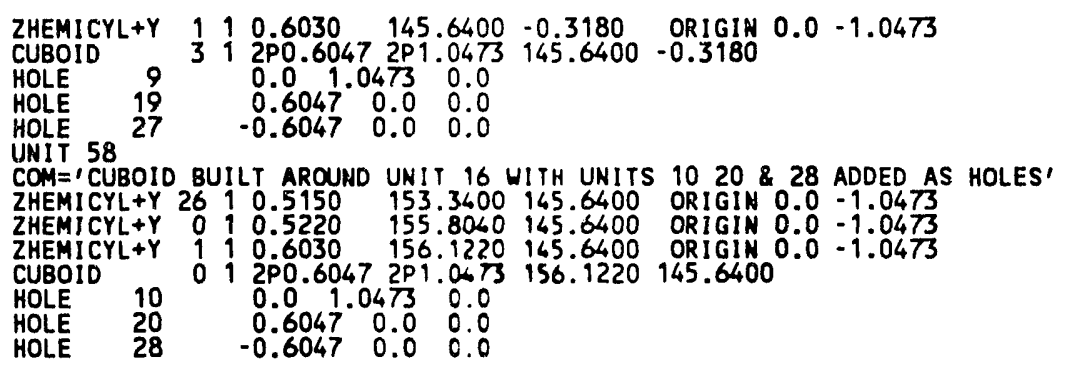

UNIT 59

COM=ICUBOID BUILT AROUND UWI" " UITM UNITS $5 \& 25$ ADDED AS HOLES

ZHEMICYL+Y 1310.5150 165.0 $x$ O 0.0 ORIGIN $0.0-1.0473$

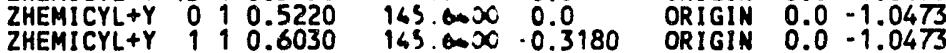

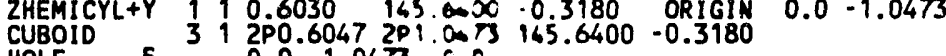

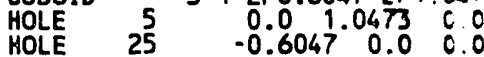

UNIT 6025

COM $=$ 'CUBOID BUILT AROUND UNIY 12 WITH UNITS $6 \& 26$ ADDED AS HOLES'

ZHEMICYL $+Y 2310.5150 \quad 153.3600145 .6400$ ORIGIN $0.0-1.0473$

2HEMICYLYY 2310.5150 153.34 145.6400 ORIGIN $0.0-1.0473$

ZHEMICYL+Y $0110.5220 \quad 155.8060145 .6400$ ORIGIN $0.0-1.0473$

$\begin{array}{lllllll}\text { ZHEMICYL+Y } & 1 & 1 & 0.6030 & 156.1220 & 145.6400 & \text { ORIGIN } \\ \text { CUBOID } & 0 & 1 & 2 \mathrm{P} 0.6047 & 2 \mathrm{P} 1.0473 & 156.1220 & 145.6400\end{array}$

$\begin{array}{lrrrrr}\text { CUBOID } & 0 & 1 & 2 P 0.6047 & 2 P 1.0675 \\ \text { HOLE } & 6 & 0.0 & 1.0473 & 0.0 \\ \text { HOLE } & 26 & -0.6047 & 0.0 & 0.0\end{array}$

UNIT 61

COM $=$ 'CUBOID BUILT AROUND UNIT 1 HITH UNITS $11 \& 17$ ADDED AS HOLES'

Z HEMICYL-Y $210.6030 \quad 145.6400-0.3180$ ORIGIN $0.0 \quad 1.0473$

CUBOID $312 \mathrm{P} 0.60472 \mathrm{P} 1.0473145 .6400-0.3180$

HOLE 11 $\quad 0.0-1.04730 .0$

$\begin{array}{lllll}\text { HOLE } & 17 & 0.6047 & 0.0 & 0.0\end{array}$

COM $=$ CUBOID BUILT AROUND UNIT 2 WITH UNITS $12 \& 18$ ADDED AS HOLES'

ZHEMICYL-Y 2 i 0.6030156 .1220145 .6400 ORIGIN 0.01 .0473

CUBOID O 1 2P0.6047 2P1.0473 156.1220145 .6400

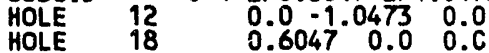

UNIT 63

COM = ' CUBOID BUILT AROUND UNIT 5 WITH UNITS $1521 \& 23$ ADDED AS HOLES'

ZHEMICYL-Y $1310.5150 \quad 145.6400 \quad 0.0000$ ORIGIN $0.0 \quad 1.0473$

ZHEMICYL-Y $010.5220 \quad 145.6400 \quad 0.0000$ ORIGIN $0.0 \quad 1.0473$

ZHEMICYL-Y $110.6030 \quad 145.6400-0.3180$ ORIGIN $0.0 \quad 1.0473$

CUBOID 312 P0.6047 2P1.0473 $145.6400-0.3180$

HOLE 15 $0.0-1.04730 .0$

$\begin{array}{lllll}\text { HOLE } & 21 & 0.6047 & 0.0 & 0.0 \\ \text { HOLE } & 23 & -0.6047 & 0.0 & 0.0\end{array}$

UNIT 64 23

COM $=$ 'CUBOID BUILT AROUND UNIT 6 WITH UNITS $1622 \& 24$ ADDED AS HOLES

ZHEMICYL-Y $23 \quad 10.5150 \quad 153.3400145 .6400$ ORIGIN $0.0 \quad 1.0473$

ZHEMICYL-Y $010.5220 \quad 155.8040 \quad 145.6400$ ORIGIN $0.0 \quad 1.0473$

ZHEMICYL-Y $110.6030 \quad 156.1220145 .6400$ ORIGIN $0.0 \quad 1.0473$

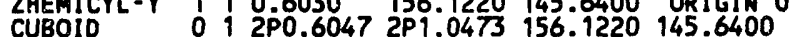

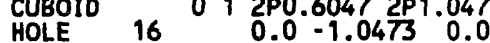

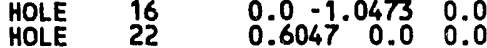

$\begin{array}{lllll}\text { HOLL } & 22 & 0.6047 & 0.0 & 0.0 \\ \text { HOLE } & 24 & -0.6047 & 0.0 & 0.0\end{array}$

UNIT 65

COM=ICUBOID BUILT AROUND UNIT 7 WITH UHITS $1529 \& 27$ ADDED AS HOLES'

ZHEMICYL-Y 1410.5150 145.6400 0.0000 ORIGIN 0.001 .0473

ZHEMICYL-Y $010.5220 \quad 145.6400 \quad 0.0000$ ORIGIN 0.01 .0473

ZHEMICYL-Y $1110.6030 \quad 145.6400 \quad-0.3180$ ORIGIN $0.0 \quad 1.0473$

$\begin{array}{lllllll}\text { CUBOID } & 312 P 0.60472 P 1.0473 & 145.6400 & -0.3180\end{array}$

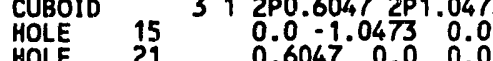

$\begin{array}{lllll}\text { HOLE } & 21 & 0.6047 & 0.0 & 0.0 \\ \text { HOLE } & 27 & -0.6047 & 0.0 & 0.0\end{array}$

UNIT 66

COM=ICUBOID BUILT AROUND UNIT 8 WITH UNITS $1622 \& 28$ ADDED AS HOLES ZHEMICYL-Y $2410.5150 \quad 153.3400145 .6400$ ORIGIN 0.01 .0473

ZHEMICYL-Y $010.5220 \quad 155.8040145 .6400$ ORIGIN $0.0 \quad 1.0473$

ZHEMICYL-Y $1110.6030 \quad 156.1220145 .6400$ ORIGIN $0.0 \quad 1.0473$

CUBOID O 12 2P0.6047 2P1.0473 156.1220145 .6400

HOLE $16 \quad 0.0-1.04730 .0$

$\begin{array}{lllll}\text { HCLE } & 22 & 0.6047 & 0.0 & 0.0 \\ \text { HOLE } & 28 & -0.6047 & 0.0 & 0.0\end{array}$

UNIT 67

COM=' CUBOID BUILT AROUND UNIT 5 WITH UNITS $1517 \& 27$ ADDED AS HOLES ZHEMICYL-Y $13,0.5150$ 145.6400 0.0000 ORIGIN 0.0190473 


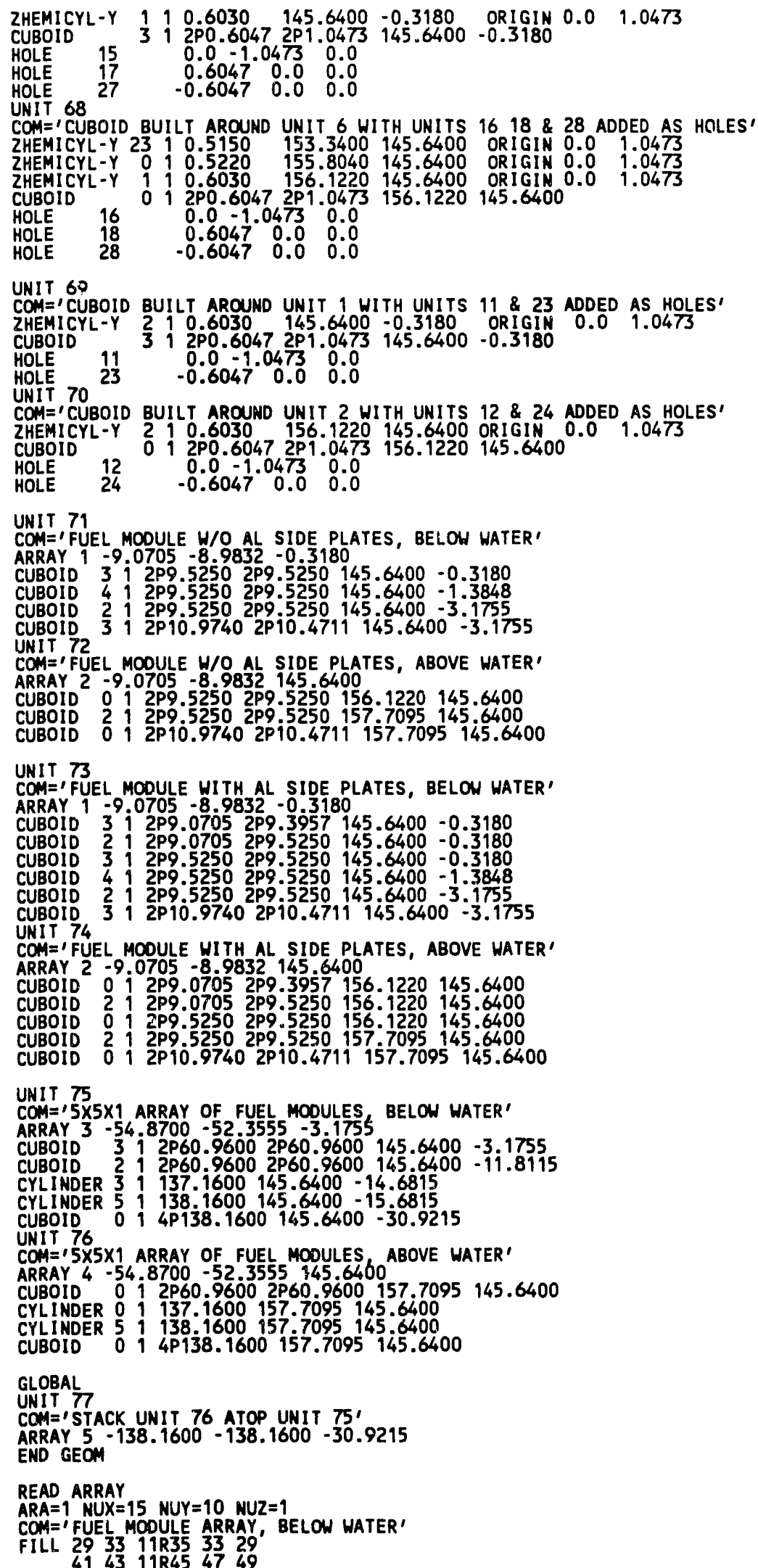




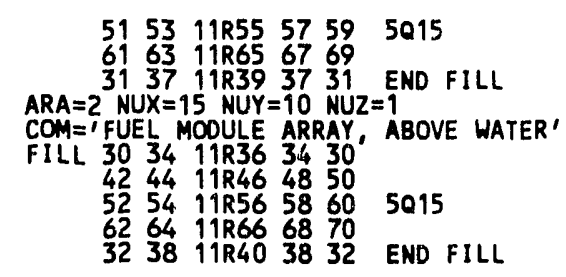

$A R A=3$ NUX $=5$ NUY $=5$ NUZ $=1$

COM=IARRAY OF FUEL MODULES BELOW WATER'

FILL

$$
\begin{aligned}
& \text { SR71 } \\
& 71 \text { 3RT3 } 71 \\
& 71 \text { SR71 } 31 \\
& \text { 3RT3 } 71 \text { END FILL }
\end{aligned}
$$

ARA $=4$ NUX $=5$ NUY1 $\quad$ NUND

COM= $=$ AARRAY OF FUEL MODULES ABOVE WATER'
FILL.

$$
\begin{array}{ll}
72 & \text { SR74 } \\
5 R 72 \\
52 \\
3 R 74 \\
3272 \\
5 R 72
\end{array}
$$

ARA $=5$ NUX $=1$ NUY $=1 \quad$ NUZ $=2$

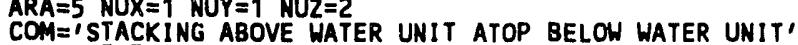
FILL 7576 END FILL

END ARRAY

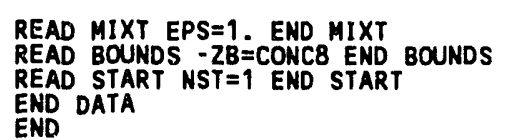

END

\section{VALIDATION CASE (CLT05)}

BAH-1645-4, EXPIMT-2485, CASE C-2-C, DANCOFF FACTOR FOR EACH FUEL PIN 27GROUPNDF4 LATTICECELL

AL 11.0 294.0 END

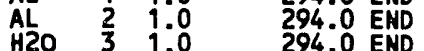

H2O 3 BON 3 3.. $3888-4294.0$ END

H2O 40.75 294.0 END

BORON $42.6166-4294.0$ END

AL 4 CARBONSTEEL $5,25294.0$ END

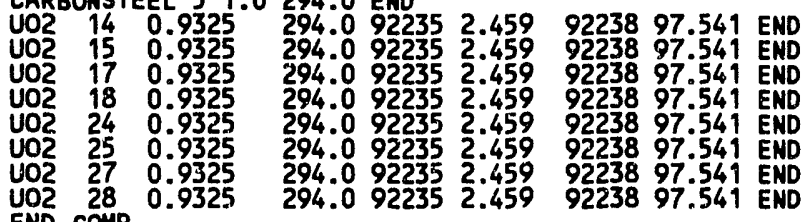

END COMP

SQUAREPITCH 1.20931 .03001831 .206011 .04400 END

MORE DATA RES $=14$ CYLINDER 0.5150 DAN (14) $=0.30655$

RES $=15$ CYLINDER 0.5150 DAN $(15)=0.43008$

RES $=17$ CYLINDER 0.5150 DAN (17) $=0.58330$

RES $=24$ CYLINDER 0.5150 DAN(24) $=0.47740$

RES $=25$ CYLINDER 0.5150 DAN(25) $=0.63128$

RES $=27$ CYLINDER 0.5150 DAN $(27)=0.85323$

BAW-1645-4, EXPIMT-2485, CASE C-2-C, DANCOFF FACTOR FOR EACH FUEL PIN READ PARAM FDN=YES NUB=YES END PARAM

READ GEOM

\section{UNIT 1}

COM=IAL PIN BELON MATER

CYLINDER $2100.6030 \quad 145.0000-0.3180$

CUBOID 314 P0.6047 $145.0000-0.3180$

UNIT 2

COM $=$ 'AL PIN ABOVE HATER'

CYLINDER 210.6030156 .1220145 .0000

CUBOID 014 P0.6047 156.1220 145.0000

\section{UNIT 3}

COM $=$ FUEL PIN BELOW WATER SURRONDED BY 4 PINS'

CYLINDER 1410.5150145 .00000 .0

CYLINDER 0110.5220145 .00000 .0

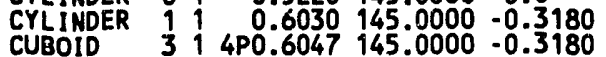


UNIT 4

COM='FUEL PIN ABOVE WATER, SURRONDED BY 4 PINS' CYLINDER 241 T 0.5150153 .3400145 .0000

CYLINDER O $1 \quad 0.5220155 .8040145 .0000$

CYLINDER i $1 \quad 0.6030156 .1220145 .0000$

CUBOID O 14 P0.6047 156.1220 145.0000

UNIT

COM=' FUEL PIN BELOW WATER SURRONDED BY 5 PINS'

CYLINDER 1510.5150145 .00000 .0

CYLINDER $010.5220145 .0000 \quad 0.0$

CYLINDER 11 0.6030 $145.0000-0.3180$

CUBOID 3 I 4 P0.6047 $145.0000=0.3180$

UNIT 6

COM=' FUEL PIN ABOVE HATER SURRONDED BY 5 PINS'

CYLINDER 251 0.5150153 .3400145 .0000

CYLINDER 010.5220155 .8040145 .0000

CYLINDER 110.6030156 .1220145 .0000

CUBOID 014 P0.6047 156.1220145 .0000

UNIT 7

COMIT I FUEL PIN BELOW WATER SURRONDED BY 7 PINS'

CYLINDER 1790.5150145 .00000 .0

CYLINDER 010.5220145 .00000 .0

CYLINDER 1 1 $0.6030145 .0000-0.3180$

CUBOID $3 \uparrow 4$ PO.6047 $145.0000=0.3180$

UNIT 8

COM= ' FUEL PIN ABOVE MATER SURRONDED BY 7 PINS'

CYLINDER 2710.5150153 .3400145 .0000

CYLINDER 010.5220155 .8040145 .0000

CYLINDER 11 1 0.6030156 .1220145 .0000

CUBOID 0 T 1400.6047156 .1220145 .0000

CUBOID

UNIT 9

COM=' FUEL PIN BELOW HATER SURRONDED BY 8 PINS'

CYLINDER 1810.5150145 .00000 .0

CYLINDER $011 \quad 0.5220145 .0000 \quad 0.0$

CYLINDER 1 1 $0.0030145 .0000-0.3180$

UNIT 10

COM=' FUEL PIN ABOVE WATER SURRONDED BY 8 PINS'

CYLINDER 2810.5150153 .3400145 .0000

CYLINDER 010.5220155 .8040145 .0000

CYLINDER 19.5220155 .8040145 .0000

$\begin{array}{llllll}\text { CYLINDER } & 1 & 1 & 0.6030 & 156.1220 & 145.0000 \\ \text { CUBOID } & 0 & 1 & 4 P 0.6047 & 156.1220 & 145.0000\end{array}$

UNIT 11

COM=' FUEL MODULE W/O AL SIDE PLATE, BELOW WATER' ARRAY $1=9.0705-9.0705-0.3180$

CUBOID 3 i 4 P9.5250 $145.0000-0.3180$

CUBOID 414 PQ.5250 $145.0000-1.3848$

CUBOID $214 \mathrm{Pg} .5250145 .0000-3.1755$

CUBOID 3 I 4 P9.9595 $145.0000-3.1755$

UNIT 12

COM=1 FUEL MODULE W/O AL SIDE PLATE, ABOVE WATER'

ARRAY $2-9.0705$ - $9.0705145,0000$

CUBOID O T $_{4 P 9.5250} 156.1220145 .0000$

CUBOID 214 P9.5250 157.7095145 .0000

CUBOID 014 P9.9595 157.7095145 .0000

UNIT 13

COM=' FUEL MOOULE $W$ AL SIDE PLATE, BELOW HATER'

ARRAY $1-9.0705-9.0705-0.3180$

CUROID 31 2PQ 0705 2P9 $3957145.0000-0.3180$

CUBOID 3 2P9.0705 2P9.3957 $145.0000-0.3180$

CUBOID 212 2P9.0705 2P9.5250 $145.0000 \quad-0.3180$

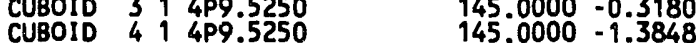

CUBOID 414 P9.5250 $\quad 145.0000-1.3848$

$\begin{array}{llllll}\text { CUBOID } & 2 & 1 & 4 \text { P9.5250 } & 145.0000 & -3.1755 \\ \text { CUBOID } 314 \text { PQ.9595 } & 145.0000 & -3.1755\end{array}$

UNIT 14

COM=I FUEL MODULE W AL SIDE PLATE, ABOVE HATER'

ARRAY $2-9.0705-9.0705145 .0000$

CUBOID $012 P 0.07052 P 93957156.1220145 .0000$

(15.070

CuBD 21289.0705 2P9.5250 156.1220145 .0000

CUBO10 156.1220145 .0000

$\begin{array}{llllll}\text { CUBOID } & 2 & 1 & 4 \mathrm{Pg} 9.5250 & 157.7095 & 145.0000 \\ \text { CUBOID } & 0 & 1 & 4 \mathrm{Pg} .9595 & 157.7095 & 145.0000\end{array}$

UNIT 15

CON $=15 \times 5$ ARRAY OF FUEL MOOULES, BELOW WATER'

ARRAY 3 -49.7975 - 49.7975 -3.1755

CUBOID 314 P $60.9600145 .0000-3.1755$

CUBOID 214 P $60.9600145 .0000-11.8115$

CYLINDER $31137.1600145 .0000-14.6815$

CYLINDER $51138.1600145 .0000-15.6815$

CYBOID 0 I 4 P $138.1600145 .0000-30.9215$

CUBOID 16

COM=15X5 ARRAY OF FUEL MODULES ABOVE WATER

ARRAY 4 - $49.7975-49.7975145 .6000$

CUBOID 0 i 4960.9600157 .7095145 .0000 
CYLINDER O $1 \quad 137.1600 \quad 157.7095 \quad 145.0000$

CYLINDER 51 138.1600 157.7095145 .0000

CUBOID 0 i 4 P 138.1600157 .7095145 .0000

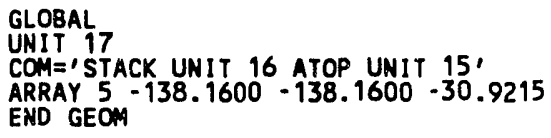

BAH-1645-4, EXPIMT-2491 CASE C-2-C DANCOFF FACTOR FOR EACH FUEL PIN RERO PARAM FDN=YES NUB=YES END PARAM

READ GEOM

COM $=\prime A L$ PIN BELOW WATER

CYLIMDER 2 1 0.6030 $100.2400-0.3180$

CUBOID 314 PO.6047 $100.2400-0.3180$

UNIT 2

COM=IAL PIN ABOVE WATER

CYLINDER 210.6030156 .1220100 .2400

CUBOID 0 i 4 P0.6047 156.1220100 .2400 
UNIT 3

COA $=$ 'FUEL PIN BEL.OW WATER SURRONDED BY 4 PINS'

CYLINDER 1410.5150106 .24000 .0

CYLINDER O $190.5220100 .2400 \quad 0.0$

CYLINDER i 1 0.6030 $900.2400-0.3180$

CUBOID 3 i 4 P0.6047 $100.2400-0.3180$

UNIT 4

COM='FUEL PIN ABOVE HATER SURRONDED BY 4 PINS'

CYLINDER 2410.5150153 .3400100 .2400

CYLINDER 0110.5220155 .8040100 .2400

CYLINDER 1 I 0.6030156 .1220100 .2400

CUBOID O 14 P0.6047 156.1220 100.2400

UNIT 5

COM=' FUEL PIN BELOW HATER SURRONDED BY 5 PINS'

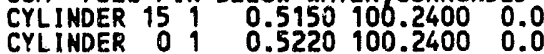

$\begin{array}{llllll}\text { CYLINDER } & 0 & 1 & 0.5220 & 100.2400 & 0.0 \\ \text { CYLINDER } & 1 & 1 & 0.6030 & 100.2400 & -0.3180\end{array}$

$\begin{array}{llllll}\text { CYLINDER } & 1 & 1 & 0.6030 & 100.2400 & -0.3180 \\ \text { CUBOID } & 3 & 1 & 4 P 0.6047 & 100.2400 & -0.3180\end{array}$

UNIT 6

COM=' FUEL PIN ABOVE GATER SURRONDED BY 5 PINS'

CYLIMDER 25 1 0.5150 153.3400100 .2400

CYLINDER D I 0.5220155 .8040100 .2400

CYLINDER \& $9 \quad 0.6030156 .1220100 .2400$

CUBOID O 1490.6047156 .1220100 .2400

COM='FUEL PIN BELOW WATER SURRONDED BY 7 PINS'

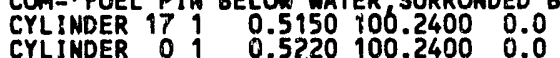

$\begin{array}{lllll}\text { CYLINDER } & 0 & 0.5220 & 100.2400 & 0.0 \\ \text { CYLIINDER } & 1 & 0.6030 & 100.2400 & -0.3180\end{array}$

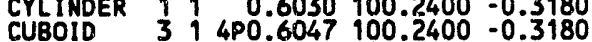

UNIT 8

COM=IFUEL PIN ABOVE HATER, SURRONDED BY 7 PINS'

CYLINDER 271 O.5150 153.3400100 .2400

CYLINDER O 10.5220155 .8040100 .2400

CYLINDER I 10.6030156 .1220100 .2400

CUBOID 014 P0.6047 156.1220 100.2400

UNIT 9

COM $=$ ' FUEL PIN BELON WATER SURRONDED BY 8 PINS'

$\begin{array}{lllll}\text { CYLINDER 18 } 1 & 0.5150 & 106.2400 & 0.0 \\ \text { CYLINDER } & 0.5220 & 100.2400 & 0.0\end{array}$

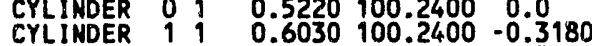

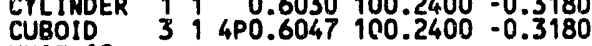

UMIT 10

COM=' FUEL PIN ABOVE WATER SURRONDED BY 8 PINS'

CYLINDER 28110.5150153 .3400100 .2400

CYLINDER O I 0.5220155 .8040100 .2400

CYLIHDER 11 o.6030 156.1220100 .2400

CUBOID O I 4 PO.6047 156.1220 100.2400

UNIT 91

COM='FUEL MOOULE W/O AL SIDE PLATE, BELOW WAIER'

ARRAY 1 -9.0705 $-9.0705-0.3180$

CUBOID 3 i 4 P9.5250 $100.2400-0.3180$

CUBOID 414 P9.5250 $100.2400-1.3848$

CUBOID 214 P9.5250 $100.2400-3.175$

CUBOID 314 P9.9595 $100.2400-3.1755$

UNIT 12

COM='FUEL MOOULE W/O AL SIDE PLATE, ABOVE HATER'

ARRAY $2-9.0705-9.0705100 .2400$

CUBOID O 14 P 9.5250 156.1220100 .2400

CUBOID 214 PO.5250 157.7095100 .2400

CUBOID 0 i 4 P9.9595 157.7095900 .2400

UNIT 13

COM='FUEL MOOULE W AL SIDE PLATE, BELOW HATER' ARRAY $1=9.0705-9.0705-0.3180$

CUBOID 3 i $2 P 0.07052 P 0.3057$ 100.2400 - 0.3180

CUBOID $212 \mathrm{2P9.0705} 2 \mathrm{PQ} .5250100 .2400-0.3180$

CUBOID 314 P9.5250 $100.2400-0.3180$

CUBOID $414 \mathrm{pg} .5250 \quad 100.2400-1.3848$

CUBOID $214905250 \quad 100.2400-3.1755$

CUBOID 3 i $499.9595 \quad 100.2400-3.1755$

CUBOID 314 4P9.9595 $100.2400-3.175$

COMIT $=14$ FUEL MOOULE $W$ AL SIDE PLATE, ABOVE WATER

ARRAY $2-9.0705-9.0705160$. 2400

CUBOID O $\mathrm{i} 2 \mathrm{2P9.0705} 2 \mathrm{PP} .3957 \quad 156.1220100 .2400$

CUBOID 212 2P9.0705 2P9.5250 156.1220 100.2400

CUBOID 014 4P9.5250 156.1220100 .2400

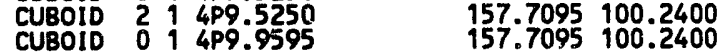

UNIT 15

COM='5X5 ARRAY OF FUEL MOOULES, BELOW HATER'

ARRAY 3 - 49.7975 - $49.7975-3.1455$

CUBOID 3 i 4 P60.9600 $100.2400-3.1755$

CUBOID 214 P $60.9600100 .2400-11.8115$

iYLINDER 3 i $137.1600100 .2400-14.6815$ 
CYLINDER $51 \quad 138.1600100 .2400-15.6815$

CUBOID $O$ I 4 P138.1600 $100.2400-30.9215$

COMIT $15 \times 5$ ARRAY OF FUEL MOOULES, ABOVE HATER'

ARRAY 4 - $49.7975-49.7975100 .2400$

CUBOID O $i$ 4P60.9600 157.7095 100.2400

CYLINDER D 1 137.1600 157.7095100 .2400

CYLINDER 51 138.1600 157.7095100 .2400

GLOBAL

UNIT IT

COM='STACK UNIT 16 ATOP UNIT 15'

ARRAY $5-138.1600-138.1600-30.9215$

END GEON

\section{READ ARRAY}

$A R A=1$ NUX $=15$ NUY $=15$ NUZ $=1$

COM=' FUEL MOOULE PIN RARAY, BELOW WATER'

FILL $\frac{1}{3} 11$ RS 31

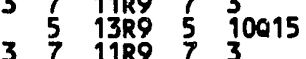

$\begin{array}{lllll}3 & 7 & 11 R 9 & 7 & 3 \\ 1 & 3 & 11 R 5 & 3 & 1\end{array}$

$A R A=2 \quad M U X=15$ MUY $=15$ MUZ $=1$ END FILL

COM $=$ I FUEL MOOULE PIN ARRAY, ABOVE WATER'

FILL 244 IIRG 42

48 11R10 84

48 11R10 8 80015

2 4 $11 R 6 \quad 4 \quad 2$

$A R A=3$ NUX $=5$ NUYG

COM $=15 \times 5$ ARRAY OF FUEI. MOOULES, BELOW HATER'

FILL 5RII

11 3R 1311

11 SR 1311

END FILL

$A R A=4$ NUX $=5$ NUY $=5$ NUZ $=1$

COM='5X5 ARRAY OF FUEL MOOULES, BELOW HATER'

FIL

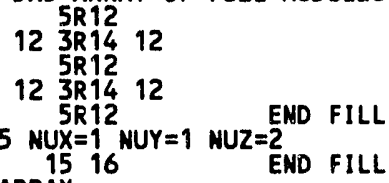

FILL 15

READ BOUNDS - ZB=CONC8 END BOUNDS

READ START NST $=1$ END START

END DATA

END

VALIDATION CASE (CLTO7)

BAH-1645-4, EXPIMT-2500, CASE C-2-C, DANCOFF FACTOR FOR EACH FUEL PIN 27GROUPNDF4 LATTICECELL

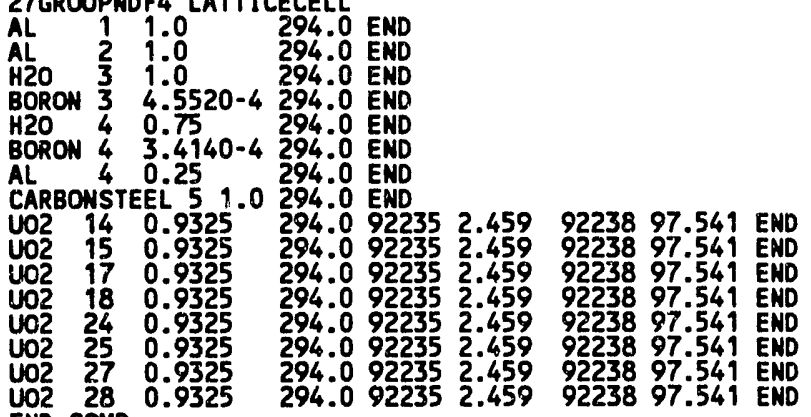

END COMP

SQUAREPITCH 1.40971 .03001831 .206019 .04400 END

MORE DATA RES $=14$ CYLINDER $0.5150^{\circ}$ DAN(14) $=0.17297$

RES $=15$ CYLINDER 0.5150 DAN(15) $=0.24270$

RES $=17$ CYLINDER 0.5150 DAN 17$)=0.32913$

RES $=24$ CYLINDER 0.5150 DAN(24) $=0.46465$

RES $=25$ CYLINDER 0.5150 DAN(25) $=0.59972$

RES $=27$ CYLINDER 0.5150 DAN(27) $=0.79067$

BAY-1645-4, EXP'MT-2500 CASE C-2-C DANCOFF FACTOR FOR EACH FUEL PIM

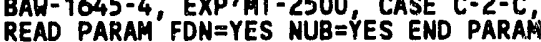

READ GEOA 
UNIT 1

COM $=$ AL PIN BELOW WATER

CYLINDER $21 \quad 0.6030 \quad 144.8500-0.3180$ CUBOID 3 I 4 PO. $.7049144 .8500-0.3180$

COM $=$ 'AL PIN ABOVE HATER'

CYLINDER 2 10.6030156 .1220144 .8500

CUBOID O 14 PO..7049 156.1220144 .8500

UNIT 3

CON=' FUEL PIN BELOW MATER, SURRONDED BY 4 PINS'

CYLINDER 141 T.5150 146.8500000

CYLINDER i 1 0.5030 $166.8500-0.3180$

CUBOID 314 PO.TOLO $146.8500-0.3180$

UNIT 4

COM='FUEL PIN ABOVE MITR SURRONDED BY 4 PINS'

CYLINDER 241 O.5130 $\$ 3 \$ .3400144 .8500$

CYLINDER 010.5220195 .8040144 .8500

CYLINDER $110.00 \% 96.1220144 .8500$

CUBOID 014 PO. TOA 156. 1220144.8500

UNIT 5

COM=' FUEL PIN BELO MIER, SURRONDED BY 5 PINS'

CYLINDER 1510.5150 164.8500 0.0

CYLINDER $011 \quad 0.5220164 .8500 \quad 0.0$

CYLINDER $11 \quad 0.6030164 .8500-0.3180$

CUBOID 314 PO.7049 $144.8500-0.3180$

UNIT 6

COMI ='FUEL PIN ABOVE WATER SURRONDED BY 5 PINS'

CYLINDER 2510.55150153 .3400144 .8500

CYLINDER 011 0.5220 155.8040144 .8500

CUBOID 014 PO.7049 156.1220 144.8500

UNIT 7

COM=' FUEL PIN BELOU UATER SURRONDED BY 7 PINS'

CYLINDER $1790.5150144 .8500 \quad 0.0$

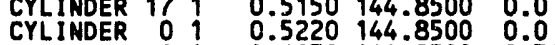

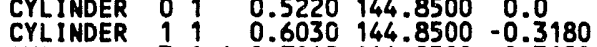

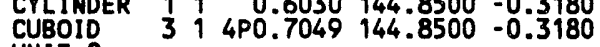

UNIT 8

CON='FUEL PIN ABOVE HATER SURRONDED BY 7 PINS'

CYLINDER 27110.5150153 .3400144 .8500

CYLINDER O 10.5220155 .8040144 .8500

CYLINDER 110.6030156 .1220144 .8500

CUBOID O 14 PO.7049 156.1220 144.8500

COM='FUEL PIN BELON WATER SURRONDED BY 8 PINS'

CYLINDER 1810.5150144 .85000 .0

CYLIMDER O $1 \quad 0.5220144 .8500 \quad 0.0$

CYLINDER i $1 \quad 0.6030144 .8500-0.3180$

CUBOID 314 PO.7049 $144.8500-0.3180$

UNIT 10

COM=' FUEL PIN ABOVE WATER SURRONDED BY 8 PINS'

CYLINDER 2810.5150153 .3400144 .8500

CYLINDER O I 0.5220155 .8040144 .8500

CYLIMDER 190.6030156 .1220144 .8500

CUBOID 014 PO.7049 156.1220 144.8500

UNIT 11

COM=' FUEL MODULE H/O AL SIDE PLATE, BELOW WATER'

ARRAY $1-9.1637$-9.1637 -0.3180

CUBOID 3 i 4 P9.5250 $144.8500-0.3180$

CUBOID $414 \mathrm{PQ} 5250144.8500-1.3848$

CU8O10 $21490.5250144 .8500-3.1755$

CUBOID 314 PQ.9578 144.8500 -3.1755

UNIT 12

COM='FUEL MODULE W/O AL SIDE PLATE, ABOVE WATER'

ARRAY $2-9.1637=9.1637144 .8500$

CUBOID O $i$ 4P9.5250 156.1220144 .8500

CUBOID 214 PQ.5250 157.7095144 .8500

CUBOID 014 P9.9578 157.7095144 .8500

UNIT 13

COM=' FUEL MOOULE $W$ AL SIDE PLATE, BELOW WATER'

ARRAY $1-9.1637-9.1637-0.3180$

CUBOID 3 T

CUBOID 3 i 2 9P.1637 2P9.395 $144.8500-0.3180$

CUBOID 212 2P9.1637 2P9.5250 $144.8500 \quad-0.3180$

CUBOID 314 P9.5250 $\quad 144.8500-0.3180$

CUBOID 414 P9.5250 $144.8500-1.3848$

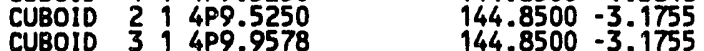

UNIT 14

COM=' FUEL MOOULE $W$ AL SIDE PLATE, ABOVE HATER'

ARRAY $2=9.1637-9.1637144 .8500$

ARRAY 2 -9.1637 -9.1637 144.8500

CUBOID 0 i $2 P 9.16372 P 9.3957 \quad 156.1220144 .8500$

CUBOID 212 P9.1637 2P9.5250 156.1220 144.8500

CUBOID 0 1 4P9.5250 156.1220144 .8500 


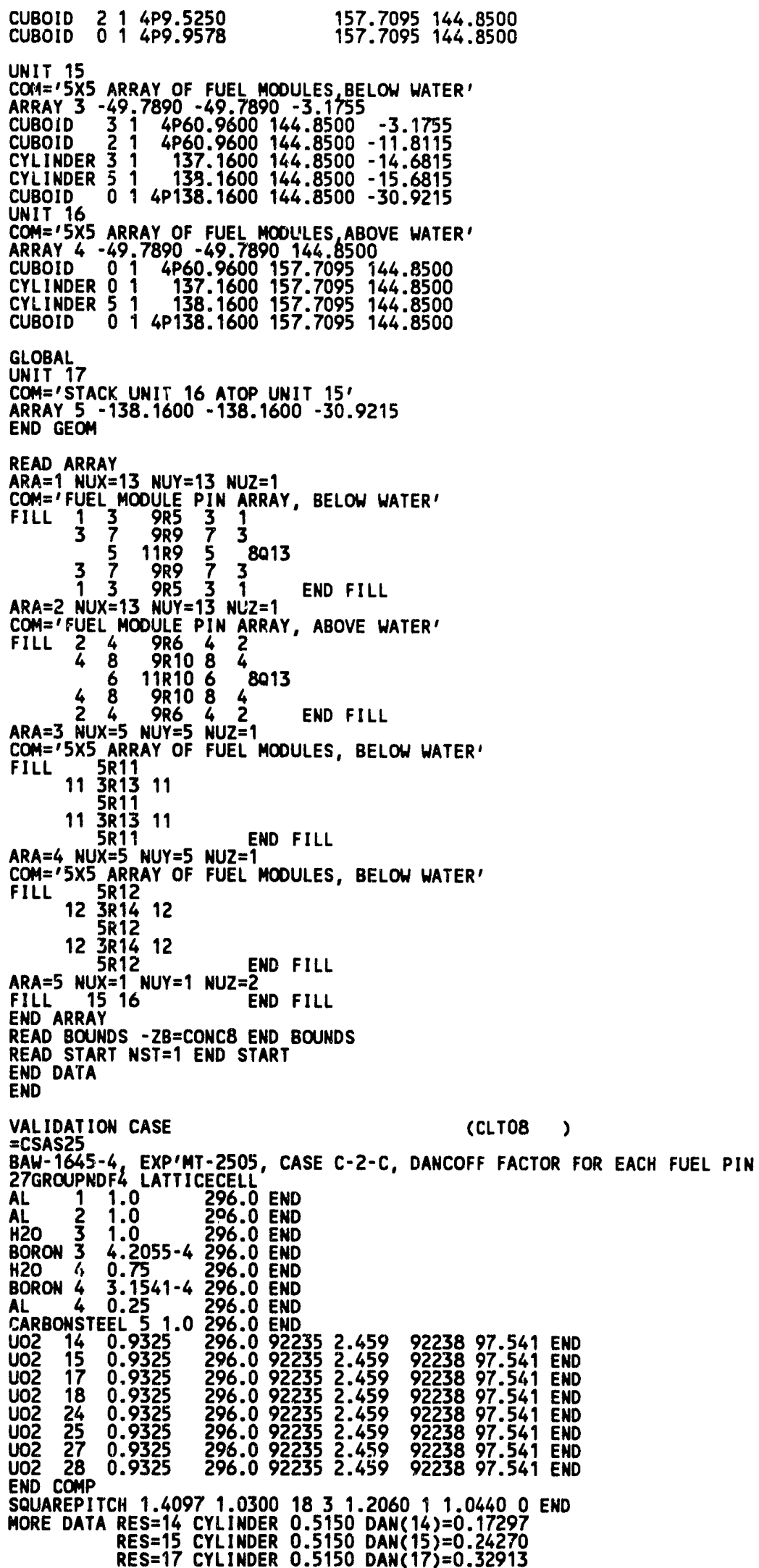


RES $=24$ CYLINDER 0.5150 DAN(24) $=0.46465$

RES $=25$ CYLINDER 0.5150 DAN $(25)=0.59972$

RES $=27$ CYLINDER 0.5150 DAN (27) $=0.79067$

RES $=28$ CYLINDER 0.5150 DAN(28)=0.98155 END MORE
BAH-1645-4, EXP'MT - 2505, CASE C-2-C DANCOFF FACTOR FOR EACH FUEL PIN READ PARAM' FDN=YES NUB =YES END PARAḾ READ GEOA

UNIT 1

COM='AL PIN BELOW HATER'

CYLINDER $210.6030101 .8100-0.3180$

CUBOID 3 T 4 PO.7049 $101.8100-0.3180$

UNIT 2

COM=IAL PIN ABOVE WATER

CYLINDER 2 140.6030156 .1220101 .8100
CUBOID 014 PO.7049 156.1220101 .8100

UNIT 3

COM='FUEL PIN BELOW HATER SURRONDED BY 4 PINS'

CYLINDER 4410.5150101 .81000 .0

CYLINDER 01 1 0.5220101 .8100000

$\begin{array}{llllll}\text { CYLINDER } & 1 & 1 & 0.6030 & 101.8100 & -0.3180 \\ \text { CUBOID } & 3 & 1 & 4 P D .7049 & 101.8100 & -0.3180\end{array}$

COMIT 4 FUEL PIN ABOVE WATER SURRONDED BY 4 PINS'

COM=' FUEL PIN ABOVE HATER SURRONDED BY 4
CYLINDER 24110.5150153 .3400101 .8100

CYLINDER 0110.5220155 .8040101 .8100

CYLINDER 11 1 0.6030156 .1220101 .8100

CUBOID 014 PO.7049 156.1220 101.8100

UNIT

COM $=$ ' FUEL PIN BELOW WATER SURRONDED BY 5 PINS'

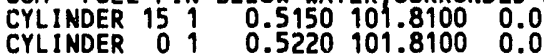

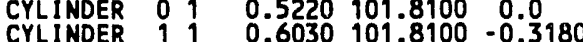

$\begin{array}{llllll}\text { CYLINDER } & 1 & 1 & 0.6030 & 101.8100 & -0.3180 \\ \text { CUBOID } & 3 & 1 & 4 P 0.7049 & 101.8100 & -0.3180\end{array}$

UNIT 6

COM=' FUEL PIN ABOVE WATER SURRONDED BY 5 PINS'

CYLINDER 251 $0.515015 \$ .3400101 .8100$

CYLINDER O 10.5220155 .8040101 .8100

CYLINDER $11 \quad 0.6030 \quad 156.1220101 .8100$

CUBOID 014 PO.7049 156.1220 101.8100

UNIT 7

COM='FUEL PIN BEI OU WATER SURRONDED BY 7 PINS'

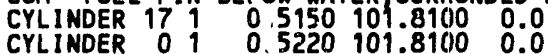

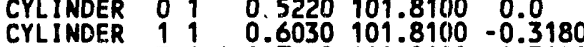

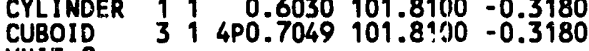

UNIT 8

COM=' FUEL PIN ABOVE WATER SURRONDED BY 7 PINS'

CYLINDER 2710.5150153 .3400101 .8100

CYLINDER 0110.5220155 .8040101 .8100

CYLINDER 110.6030156 .1220101 .8100

CUBOID O 14 PO.7049 156.1220 101.8100

UNIT

COM=I FUEL PIN BELOW WATER SURIRONDED BY 8 PINS'

CYLINDER 1810.5150101 .81000 .0

CYLINDER $010.5220101 .8100 \quad 0.0$

CYLINDER $111 \quad 0.6030101 .8100-0.3180$

CUBOID 314 PO.7049 101.8100 - 0.3180

UNIT 10

COM='FUEL PIN ABOVE WATER SURRONDED BY 8 PINS'

CYLINDER $281 \quad 0.5150153 .34 .00101 .8100$

CYLINDER 0110.5220155 .80140101 .8100

CYIINDER 110.6030156 .1220101 .8100

$\begin{array}{llllll}\text { CYLINDER } & 1 & 1 & 0.6030 & 156.1220 & 101.8100 \\ \text { CUBOID } & 0 & 1 & 4 P 0.7049 & 156.12 .20 & 101.8100\end{array}$

UNIT 11

COM='FUEL MOOULE W/O AL SIDE PLATE, BELOW WATER'

ARRAY $1=9.1637-0.1637-0.3180$

CUBOID 3 i 4 P9.5250 $101.8100-0.3180$

CUBOID 4 4P9.5250 $101.8100-1.3848$

CUBOID 214 P9.5250 $101.8100-3.1755$

CUBOID 314 P9.9578 101.8100 -3.1755

UNIT 12

COM='FUEL MODULE W/O AL SIDE PLATE, ABOVE WATER'

ARRAY 2 -9.1637 -9.1637 101.8100

CUBOID 0 1 4 P9.5250 156.1220101 .8100

CUBOID 214 PQ.5250 157.7095101 .8100

CUBOID 014 P9.9578 157.7095101 .8100

UNIT 13

COM='FUEL MODULE W AL SIDE PLATE, BELOW HATER' ARRAY $1=9.1637-9.1637=0.3180$

ARRAY

CUBOID 3 2P9.1637 2p9.3957 $101.8100-0.3180$

CUBOID 251 2P9.1637 2P9.5250 101.8100 -0.3180

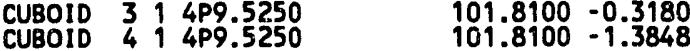




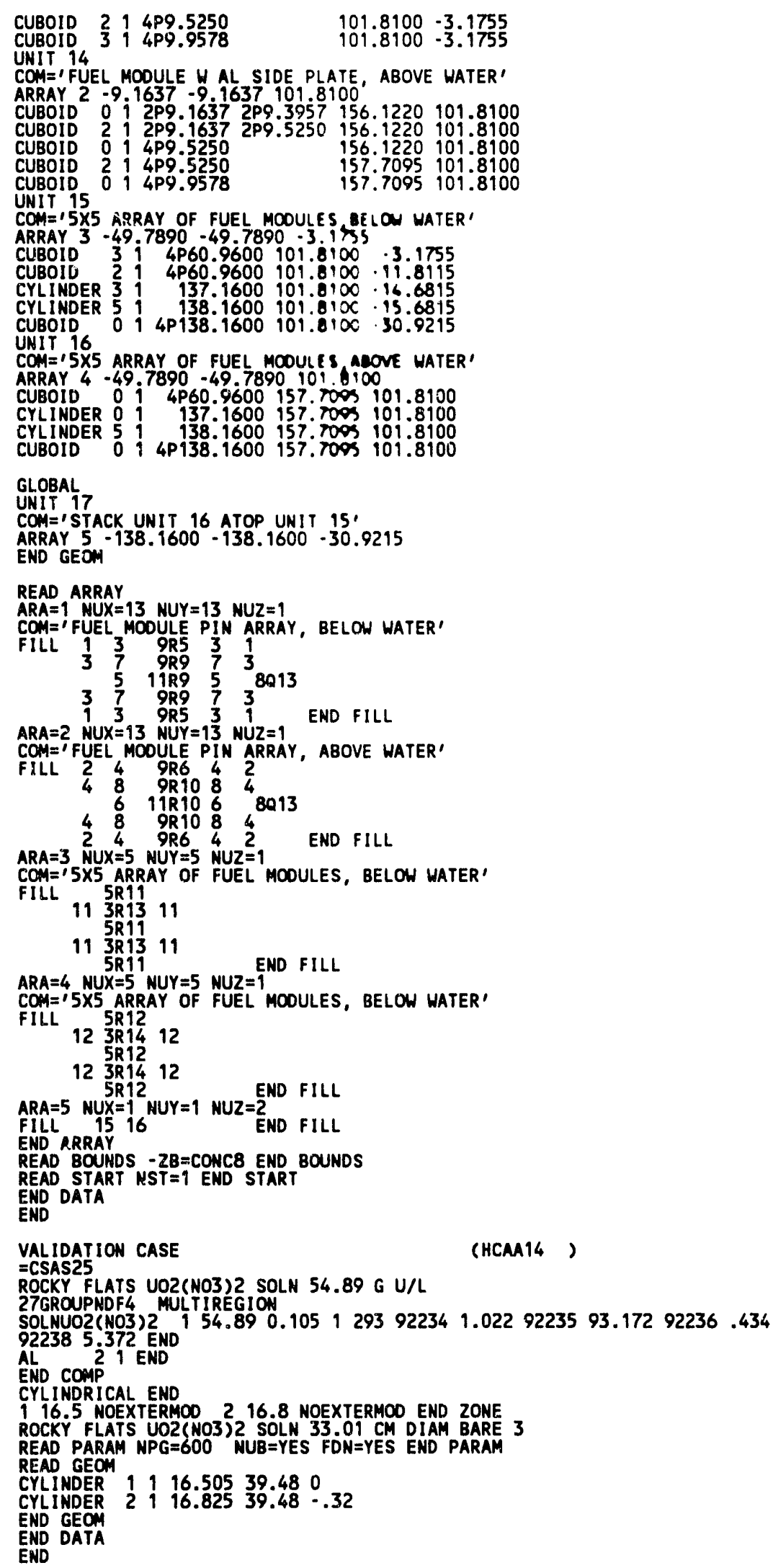


VALIDATION CASE

(HCASO2 )

=CSAS25

KEYO-5 VALIDATION CASE A-10

27GROUPNDF 4 MULTIREGION

URANIUM 1.98662939223597 .67922382 .33 END

H2O 2 END

PLEXIGLASS 3 END

END COMP

SPHERICAL END

96.55 ONEEXTERMOO 2 12.7 NOEXTERMOD END ZONE

KENO-5 VALIDATION CASE A-10

READ PARAM TME $=60$ NPG $=600$ FDN $=$ YES $A U B=Y E S$ END PARAM

READ GEOM

BOX TYPE 1

SPHERE 116.5537

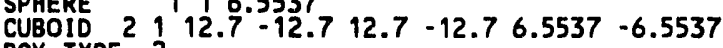

BOX TYPE 2

CYLINDER $214.12751 .27-1.27$

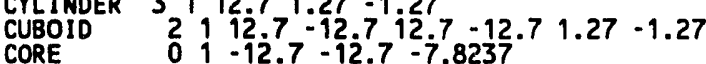

CORE

CYLINDER $2130.48 \quad 24.3337-39.5737$

END GEOH

READ ARRAY NUX $=1$ NUY $=1$ NUZ $=2$ LOOP

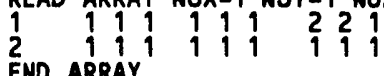

END ARRAY

END DATA

VALIDATION CASE

(HCASO6)

$=$ CSAS25

KENO-5 VALIDATION CASE A-4

SOLNUO2(NO3) 2 1 20.15 .13912939223593 .18922386 .82 END

AL

END COMP

SPHERICAL END

134.6 ONEEXTERMOD 234.92 NOEXTERMOD END ZONE

KENO-5 VALIDATION CASE A-4

READ PARAM NPG $=600$ FDN=YES NUB=YES END PARAM

READ GEOM

SPHERE

SPHERE

SPHERE 2134.6

END GEOM

EMD DATA

EHD

VALIDATION CASE

(HCASO7)

=CSAS25

KENO- 5 VALIDATION CASE $A-5$

27 GROUPNDF 4 MULTIREGIOW

URANIUM 1 9848 2839223593.5922386 .5 END

$\mathrm{H} 2 \mathrm{O}$

END COMP

SPHERICAL END

1 12.7 ONEEXTERMOD 231 . NOEXTERMOD END ZONE

KENO-5 VALIDATION CASE A-5

READ PARAM TME $=60$ NPG $=600$ FDN=YES NUB=YES END PARAM

READ GEOM

HEMISPHERE

HEMISPHER 2112.7

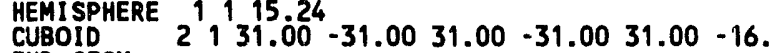

END GEOM

END DATA

END

VALIDATION CASE

(HCAS11)

KENO-5 VALIDATIOH CASE A-9

27GROUPNDF4 MULTIREGION 26.82100113 .1616000 .021 END

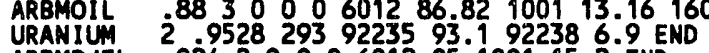

ARBMPJEL .024 20006012851001152 END

CARBONSTEEL 3 END

END COMP

SPHERICAL END

2 8.2 ONEEXTERMOO 140.5 NOEXTERMOO END ZONE KENO-5 VALIDATION CASE A-9

READ PARAM
TME $=60$ NPG $=600$ FDN $=$ YES NUB $=$ YES END PARAM

TMEAD $=60$ GEOM

HEMISPHERE

HEMI SPHERE $2^{3}, 1,0.012$

SPHERE $1140.4 \$ 8$

CUBE $0149.0-41.0$ 
END GEOM

END DATA

EHD

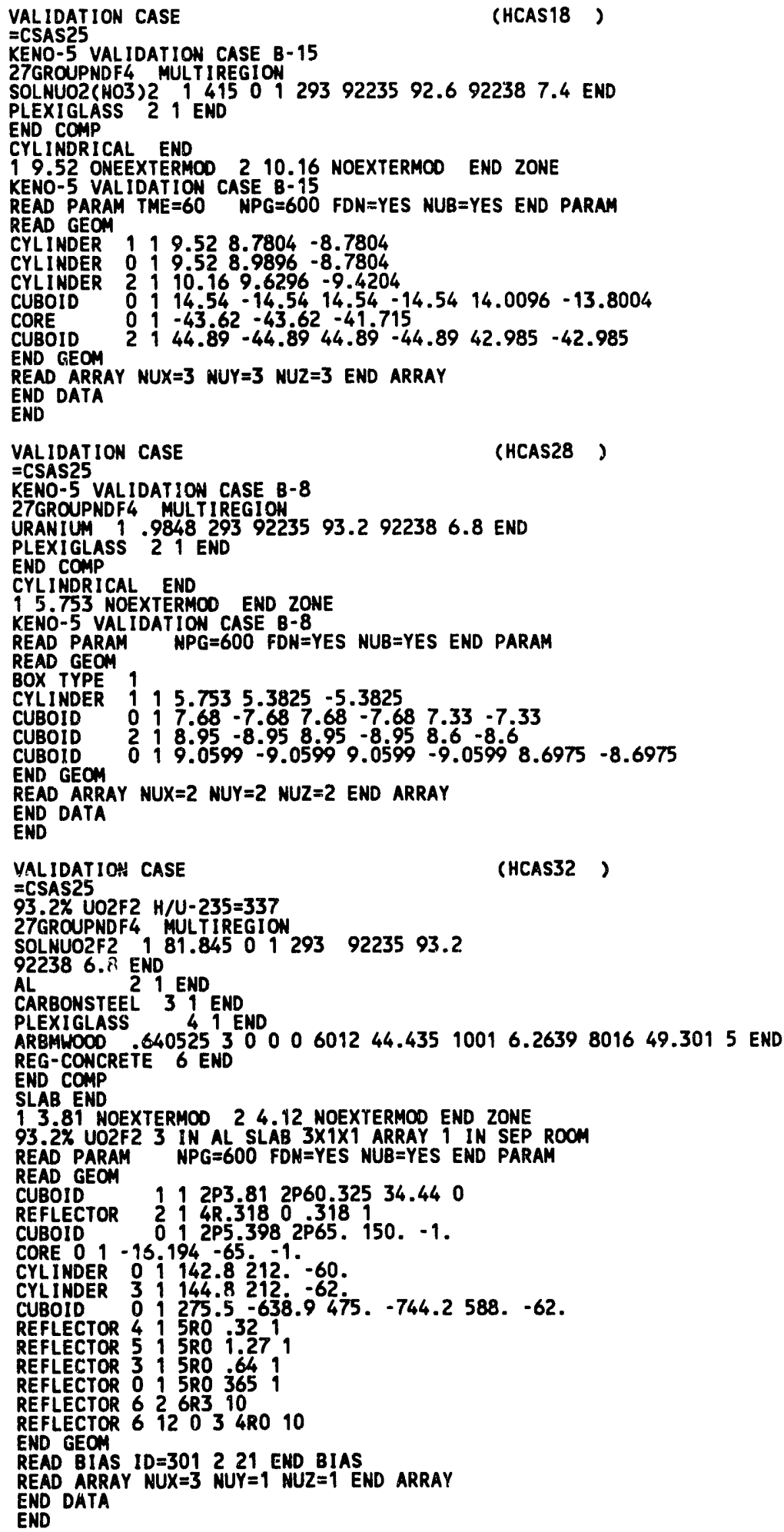


VALIDATION CASE

(HCAS52)

$=$ CSAS2

93.2\% U02F2 H/U-235=337

27GROUPNDF 4 MULTIREGION

SOLNUO2F2 181.845012939223593 .2

922386.8 END

AL

21 END

END COMP

17.62 NOEXTERMOO 27.94 NOEXTERMOO END ZONE

93. $2 \%$ UO2F2 6 \& 3 IN AL SLABS $2 \times 1 \times 1$ ARRAY 6 IN SEP

READ PARAM NPG $=600$ FDN=YES NUB=YES END PARAM

READ GEOM

UNIT 1

CUBOID

REFLECTOR

CUBOID

UNIT 2

$112 P 3.812 P 60.32545 .030$

CUBOID

$14 R .3180 \quad .318$

$012 \mathrm{P} 4.128$ 2P65. 150. -1

CUBOID

REFLECTOR

CUBOID

UNIT

$212 P .318 \quad 2 P 60.32545 .030$

UNIT

$112 P 7.9382 P 60.32545 .030$

$214 R .3180 .3181$

0 1 2 P8.574 2 Pि65. 150. -1

END GEOM

$0115.2402 P 65.150 .-1$.

READ ARRAY NUX=3 NUY=1 NUZ=1 FILL 231 END FILL END ARRAY

END DATA

END

VALIDATION CASE

$=$ CSAS25

(HCAS60)

UO2(NO3)2 279 G U/L

27GROUPNDF4 MULTIREGION

SOLNUD2(NO3)2 1279012939223592.6922387 .4 END

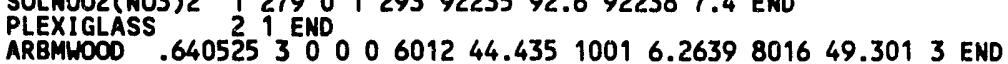

ARBMWOOD .6405253

CARBONSTEEL 4 END

END COMP

CYLINDRICAL END

19.52 ONEEXTERMOD 210.16 NOEXTERMOD END ZONE

U02(NO3)2 279 G U/L $3 \times 3 \times 3$ ARRAY UNREFL. WALLS TANK \& FLOOR

READ PARAM NPG $=600$ FDN $=$ YES NUB=YES PLT $T=N O$ END PARAM

READ GEO

UNIT 1

CYLINDER $1919.52 \quad 17.56090$

CYLINDER 0199.5217 .770 .

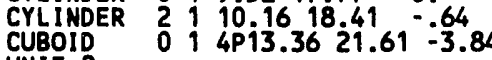

UNIT 2

ARRAY 1 2R-40.08 0

CUBOID 0 1 4P200. 250 . -50 .

UNIT 3

CYLINDER 011 142.8 224. -48 .

CYLINDER $41144.8224^{\circ}-50^{\circ}$

CUBOID $01218.1-181.9215$. 185 . 250. -50

ARRAY 2 3RO.

REFLECTOR 0 i 57.457 .260 .559 .2400 .0 .1

REFLECTOR $215 R 0^{\circ} .321$

REFLECTOR 3 \& 5 RO 1.27

REFLECTOR $415 R 0$ o 1

REFLECTOR 0 1 5R0 365

REFLECTOR $526 R 310$

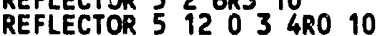

REFLECTON

READ START XSM=160 XSP $=240 \quad Y S M=160 \quad Y S P=240 \quad Z S M=50 \quad Z S P=127$ END START

READ BIAS ID $=301221$ END BIAS

READ ARRAY ARA $=1$ NUX $=3$ NUY $=3$ NUZ $=3$ FILL F1 END FILL

$A R A=2$ MUX $=2$ NUY $=1$ MUZ $=1$ FILL 23 END FILL END ARRAY

READ PLOT PLT $=$ HO

$X U L=113.2$ YUL $=286.8 \quad Z U L=60 . X L R=286.8 \quad Y L R=113.2 \quad 2 L R=60$.

UAX $=1$ VON $=-1$ MAX $=125$ END

XUL $=437.1$ YUL $=329.8$ ZUL $=60 . X L R=726.7$ YLR=40.2 ZLR=60. END

XUL $=437$.

END DATA

END

VALIDATION CASE

$=$ CSAS25

(HCAS74)

U02(NO3)2 $415 \mathrm{G} \mathrm{U} / \mathrm{L}$

77GROUPNDF4 MULTIREION

SOLNUO2(NO3)2 1415 O 12939223592.6922387 .4 END

PLEXIGLASS 21 END

$\begin{array}{lll}\text { PLEXIGLASS } & 21 \text { END } \\ \text { PARA(H2O) } & 31 \text { END }\end{array}$

END COMP

CYLINDRICAL END

19.52 ONEEXTERMOO 2 10.16 NOEXTERMOD END ZONE 
U02 (N03)2 415 G U/L 3X3X3 ARRAY 15.24 CM PARAFFIN REFL.

READ PARAM NPG $=600$ FDN=YES NUB=YES END PARAM

READ GEOM

CYLINDER 119.5217 .56090$.

CYLINDER 019.5217 .770$.

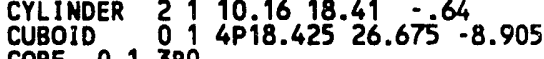

CORE $013 R O$.

REFLECTOR 3 i $6 R 34$

REFLECTOR 36 6R3.24 1

END GEOM

READ BIAS ID $=40026$ END BIAS

READ ARRAY NUX $=3$ NUY $=3$ NUZ $=3$ END ARRAY

END DATA

END 


\section{APPENDIX B. CALCULATED RESULTS AND EXPERIMENTAL PARAMETERS}

In order to analyze the data from the validation calculations, the experiments were parameterized. The results could then be categorized in a number of ways to test for trends and bias. Table B.1 contains the parameters which we a used for data analysis for each experiment.

Key terms are as follows:

RUN - Sequential number assigned to experiments.

MEMB - Case number which corresponds to Tables 1-6.

KEFF - KENO calculated $k$.

SDM - KENO calculated standard deviation.

AEG - Average energy group of neutron causing fission.

ENRLEV - Enrichment level (high, intermediate, low).

ASSAY - Fuel enrichment.

REFLCOND - Reflection condition (reflected, partially reflected, unreflected).

REFL - Reflector material.

SYSGEOM - Overall system geometry (array, single unit).

UNITGEOM - Principal geometry of unit (cylinder, slab, sphere).

FUELMIX - Indicator as to whether the system was considered primarily heterogeneous or homogeneous.

FUEL - Primary form of fissile material.

FMATL - Principal fissile nuclide in fuel.

REF - Reference number for reference which describes the critical experiment. 
New Data-.....-.Nov. 13, 1990

\begin{tabular}{|c|c|c|c|c|c|c|c|c|c|c|c|c|}
\hline MEMB & KEFF & SDM & AEG & ENRLEV & ASSAY & REFLCOND & REFL $S$ & SYSGEOH & UNITGEON & FUELMIX & FUEL & FMATL \\
\hline $\begin{array}{l}\text { CAA01 } \\
\text { CAA04 } \\
\text { CAA07 } \\
\text { CAA12 } \\
\text { CAA14 } \\
\text { CAA19 } \\
\text { CAA21 } \\
\text { CAA25 } \\
\text { CAA27 } \\
\text { CAA29 } \\
\text { CAA33 } \\
\text { CAA38 }\end{array}$ & $\begin{array}{l}0.98944 \\
0.98931 \\
0.98055 \\
0.97925 \\
0.98931 \\
0.99127 \\
0.99969 \\
0.99988 \\
0.98726 \\
0.98595 \\
0.99157 \\
1.00000\end{array}$ & $\begin{array}{l}0.00396 \\
0.00319 \\
0.00315 \\
0.00253 \\
0.00319 \\
0.00356 \\
0.00331 \\
0.00324 \\
0.00340 \\
0.00310 \\
0.00241 \\
0.00254\end{array}$ & $\begin{array}{l}21.1691 \\
23.1187 \\
21.3937 \\
24.0354 \\
23.1187 \\
23.3604 \\
24.5658 \\
24.1875 \\
22.8547 \\
24.3732 \\
24.7165 \\
24.7870\end{array}$ & $\begin{array}{l}L \\
L \\
L \\
L \\
L \\
L \\
L \\
L \\
L \\
L\end{array}$ & $\begin{array}{l}4.89 \\
4.89 \\
4.89 \\
4.89 \\
4.89 \\
4.89 \\
4.89 \\
4.89 \\
4.89 \\
4.89 \\
4.89 \\
4.89\end{array}$ & $\begin{array}{l}R \\
R \\
R \\
R \\
R \\
U \\
U \\
U U \\
U \\
U \\
U \\
R\end{array}$ & $\begin{array}{l}\mathrm{H} 2 \mathrm{O} \\
\mathrm{H} 2 \mathrm{O} \\
\mathrm{PB} \\
\text { UO2F2 } \\
\text { UO2F2 } \\
\\
\mathrm{H} 2 \mathrm{O} \\
\mathrm{H} 2 \mathrm{O} \\
\mathrm{H} 2 \mathrm{O} \\
\mathrm{H} 2 \mathrm{O}\end{array}$ & $\begin{array}{l}A \\
A \\
A \\
A \\
A \\
S \\
S \\
S \\
S \\
S \\
S \\
S\end{array}$ & $\begin{array}{l}C Y \\
C Y \\
C Y \\
C Y \\
C Y \\
S L \\
S L \\
S L \\
S L \\
S L \\
S P \\
S P\end{array}$ & $\begin{array}{l}\text { HET } \\
\text { HET } \\
\text { HET } \\
\text { HET } \\
\text { HET } \\
\text { HOM } \\
\text { HOM } \\
\text { HOM } \\
\text { HOW } \\
\text { HOM } \\
\text { HOM } \\
\text { HOM }\end{array}$ & $\begin{array}{l}\text { METAL } \\
\text { METAL } \\
\text { METAL } \\
\text { METAL } \\
\text { METAL } \\
\text { U308 } \\
\text { U308 } \\
\text { U308 } \\
\text { U308 } \\
\text { U308 } \\
\text { UO2F2 } \\
\text { UO2F2 }\end{array}$ & $\begin{array}{l}\text { U235 } \\
\text { U235 } \\
\text { U235 } \\
\text { U235 } \\
\text { U235 } \\
\text { U235 } \\
\text { U235 } \\
\text { U235 } \\
\text { U235 } \\
\text { U235 } \\
\text { U235 } \\
\text { U235 }\end{array}$ \\
\hline $\begin{array}{l}\text { CAB03 } \\
\text { CAB08 } \\
\text { CAB11 } \\
\text { CAB15 }\end{array}$ & $\begin{array}{l}0.99246 \\
0.99321 \\
1.00168 \\
1.00502\end{array}$ & $\begin{array}{l}0.00239 \\
0.00295 \\
0.00270 \\
0.00294\end{array}$ & $\begin{array}{l}16.7469 \\
15.5959 \\
17.1817 \\
18.5659\end{array}$ & $\begin{array}{l}L \\
L \\
L\end{array}$ & $\begin{array}{l}3.85 \\
3.85 \\
3.85 \\
3.85\end{array}$ & $\begin{array}{l}\mathbf{R} \\
\mathbf{R} \\
\mathbf{R} \\
\mathbf{R}\end{array}$ & $\begin{array}{l}H 2 O \\
H 2 O \\
H 2 O \\
H 2 O\end{array}$ & $\begin{array}{l}A \\
A \\
A \\
A\end{array}$ & $\begin{array}{l}c Y \\
c Y \\
c Y \\
C Y\end{array}$ & $\begin{array}{l}\text { HET } \\
\text { HET } \\
\text { HET } \\
\text { HET }\end{array}$ & $\begin{array}{l}\text { METAL } \\
\text { METAL } \\
\text { METAL } \\
\text { METAL }\end{array}$ & $\begin{array}{l}\text { U235 } \\
\text { U235 } \\
\text { U235 } \\
\text { U235 }\end{array}$ \\
\hline $\begin{array}{l}\text { CASO4 } \\
\text { CAS066 } \\
\text { CAS11 } \\
\text { CAS13 } \\
\text { CAS16 } \\
\text { CAS17 } \\
\text { CAS21 } \\
\text { CAS23 } \\
\text { CAS26 } \\
\text { CAS29 } \\
\text { CAS31 } \\
\text { CAS33 } \\
\text { CAS34 } \\
\text { CAS36 }\end{array}$ & $\begin{array}{l}0.98537 \\
0.98605 \\
0.99445 \\
0.99878 \\
0.98975 \\
0.98853 \\
1.00663 \\
1.01240 \\
1.007233 \\
1.00697 \\
1.00426 \\
0.99340 \\
0.99148 \\
0.99546\end{array}$ & $\begin{array}{l}0.00225 \\
0.00202 \\
0.00270 \\
0.00278 \\
0.00250 \\
0.00272 \\
0.00307 \\
0.00315 \\
0.00282 \\
0.00324 \\
0.00292 \\
0.00275 \\
0.00305 \\
0.00316\end{array}$ & $\begin{array}{l}23.2734 \\
23.2791 \\
22.4849 \\
23.2174 \\
23.9037 \\
24.1057 \\
22.2061 \\
22.2218 \\
21.7608 \\
23.5224 \\
23.2732 \\
24.2199 \\
24.2248 \\
24.2270\end{array}$ & $\begin{array}{l}L \\
L \\
L \\
L \\
L \\
L \\
L \\
L \\
L \\
L \\
L\end{array}$ & $\begin{array}{l}1.40 \\
1.40 \\
2.00 \\
2.00 \\
2.00 \\
2.00 \\
3.00 \\
3.00 \\
3.00 \\
3.00 \\
3.00 \\
4.98 \\
4.98 \\
4.98\end{array}$ & $\begin{array}{l}U \\
U \\
\mathbf{R} \\
\mathbf{R} \\
\mathbf{R} \\
\mathbf{R} \\
\mathbf{R} \\
\mathbf{R} \\
\mathbf{U} \\
\mathbf{R} \\
\mathbf{U} \\
\mathbf{R} \\
\mathbf{R} \\
\mathbf{U}\end{array}$ & $\begin{array}{l}\text { PARA } \\
\text { PARA } \\
\text { PARA } \\
\text { PARA } \\
\text { PARA } \\
\text { PARA } \\
\text { POLY } \\
\text { COMP } \\
\text { COMP }\end{array}$ & $\begin{array}{l}\text { S } \\
\text { S } \\
\text { S } \\
\text { S } \\
\text { S } \\
\text { S } \\
\text { S } \\
\text { S } \\
\text { S } \\
\text { S } \\
\text { S } \\
\text { S } \\
\text { S } \\
\text { S }\end{array}$ & $\begin{array}{l}\text { SL. } \\
\text { SL } \\
\text { SL } \\
\text { SL } \\
\text { SL } \\
\text { SL } \\
\text { SL } \\
\text { SL } \\
\text { SL } \\
\text { SL } \\
\text { SL } \\
C Y \\
C Y \\
C Y\end{array}$ & $\begin{array}{l}\text { HOW } \\
\text { HOW } \\
\text { HOW } \\
\text { HOM } \\
\text { HOM } \\
\text { HOW } \\
\text { HOW } \\
\text { HOW } \\
\text { HOW } \\
\text { HOW } \\
\text { HOW } \\
\text { HOW } \\
\text { HOW } \\
\text { HOM }\end{array}$ & $\begin{array}{l}\text { UF4 } \\
\text { UF4 } \\
\text { UF4 } \\
\text { UF4 } \\
\text { UF4 } \\
\text { UF4 } \\
\text { UF4 } \\
\text { UF4 } \\
\text { UF4 } \\
\text { UF4 } \\
\text { UF4 } \\
\text { UO2F2 } \\
\text { UO2F2 } \\
\text { UO2F2 }\end{array}$ & $\begin{array}{l}\text { U235 } \\
\text { U235 } \\
\text { U235 } \\
\text { U235 } \\
\text { U235 } \\
\text { U235 } \\
\text { U235 } \\
\text { U235 } \\
\text { U235 } \\
\text { U235 } \\
\text { U235 } \\
\text { U235 } \\
\text { U235 } \\
\text { U235 }\end{array}$ \\
\hline $\begin{array}{l}\text { CAR01 } \\
\text { CAR04 } \\
\text { CARO7 } \\
\text { CAR08 } \\
\text { CAR11 } \\
\text { CAR13 } \\
\text { CAR15 } \\
\text { CAR16 } \\
\text { CAR18 } \\
\text { CAR20 }\end{array}$ & $\begin{array}{l}1.01259 \\
1.01155 \\
1.01583 \\
1.00169 \\
1.00698 \\
1.00808 \\
0.99945 \\
1.00896 \\
1.00868 \\
1.00356\end{array}$ & $\begin{array}{l}0.00308 \\
0.00320 \\
0.00366 \\
0.00352 \\
0.00325 \\
0.00296 \\
0.70302 \\
0.00342 \\
0.00312 \\
0.00263\end{array}$ & $\begin{array}{l}21.3757 \\
21.1831 \\
18.3377 \\
19.2762 \\
21.5422 \\
20.11769 \\
18.5301 \\
19.3123 \\
21.8624 \\
18.3286\end{array}$ & $\begin{array}{l}L \\
L \\
L \\
L \\
L \\
L \\
L\end{array}$ & $\begin{array}{l}4.46 \\
4.46 \\
4.46 \\
4.46 \\
4.46 \\
4.46 \\
4.46 \\
4.46 \\
4.46 \\
4.46\end{array}$ & $\begin{array}{l}\mathbf{R} \\
\mathbf{R} \\
\mathbf{R} \\
\mathbf{R} \\
\mathbf{R} \\
\mathbf{R} \\
\mathbf{R} \\
\mathbf{R} \\
\mathbf{R} \\
\mathbf{R}\end{array}$ & $\begin{array}{l}\text { PLEX } \\
\text { CONC } \\
\text { PLEX } \\
\text { PLEX } \\
\text { PLEX } \\
\text { PLEX } \\
\text { PLEX } \\
\text { PLEX } \\
\text { PLEX } \\
\text { PLEX }\end{array}$ & $\begin{array}{l}\mathbf{S} \\
\mathbf{S} \\
\mathbf{S} \\
\mathbf{S} \\
\mathbf{S} \\
\mathbf{S} \\
\mathbf{S} \\
\mathbf{S} \\
\mathbf{S} \\
\mathbf{S}\end{array}$ & $\begin{array}{l}S L \\
S L \\
S L \\
S L \\
S L \\
S L \\
S L \\
S L \\
S L \\
S L\end{array}$ & $\begin{array}{l}\text { HET } \\
\text { HET } \\
\text { HOM } \\
\text { HOW } \\
\text { HET } \\
\text { MET } \\
\text { HOM } \\
\text { HOM } \\
\text { HET } \\
\text { HOM }\end{array}$ & $\begin{array}{l}\text { U308 } \\
\text { U308 } \\
\text { U308 } \\
\text { U308 } \\
\text { U308 } \\
\text { U308 } \\
\text { U308 } \\
\text { U308 } \\
\text { U308 } \\
\text { U308 }\end{array}$ & $\begin{array}{l}\text { U235 } \\
\text { U235 } \\
\text { U235 } \\
\text { U235 } \\
\text { U235 } \\
\text { U235 } \\
\text { U235 } \\
\text { U235 } \\
\text { U235 } \\
\text { U235 } \\
\text { U235 }\end{array}$ \\
\hline $\begin{array}{l}\text { CLTO1 } \\
\text { CLTO2 } \\
\text { CLTO3 } \\
\text { CLT04 } \\
\text { CLTO5 } \\
\text { CLTO6 } \\
\text { CLTO7 } \\
\text { CLT08 }\end{array}$ & $\begin{array}{l}0.98300 \\
0.97715 \\
0.98178 \\
0.99676 \\
0.98644 \\
0.98733 \\
1.00191 \\
0.99282\end{array}$ & $\begin{array}{l}0.00352 \\
0.00375 \\
0.00310 \\
0.00429 \\
0.00306 \\
0.00340 \\
0.00304 \\
0.00370\end{array}$ & $\begin{array}{l}19.2219 \\
19.1577 \\
19.7538 \\
20.2683 \\
19.8747 \\
19.7891 \\
21.5533 \\
21.4604\end{array}$ & $\begin{array}{l}L \\
L \\
L \\
L \\
L \\
L\end{array}$ & $\begin{array}{l}2.459 \\
2.459 \\
2.459 \\
2.459 \\
2.459 \\
2.459 \\
2.459 \\
2.459\end{array}$ & $\begin{array}{l}\mathbf{R} \\
\mathbf{R} \\
\mathbf{R} \\
\mathbf{R} \\
\mathbf{R} \\
\mathbf{R} \\
\mathbf{R} \\
\mathbf{R}\end{array}$ & $\begin{array}{l}\mathrm{H} 2 \mathrm{O} \\
\mathrm{H2O} \\
\mathrm{H2O} \\
\mathrm{H2O} \\
\mathrm{H2O} \\
\mathrm{H2O} \\
\mathrm{H2O} \\
\mathrm{H} 2 \mathrm{O}\end{array}$ & $\begin{array}{l}\hat{A} \\
\hat{A} \\
\hat{A} \\
\hat{A} \\
\hat{A} \\
\hat{A} \\
\hat{A}\end{array}$ & $\begin{array}{l}C Y \\
C Y \\
C Y \\
C Y \\
C Y \\
C Y \\
C Y \\
C Y\end{array}$ & $\begin{array}{l}\text { HET } \\
\text { HET } \\
\text { HET } \\
\text { HET } \\
\text { HET } \\
\text { HET } \\
\text { HET } \\
\text { HET }\end{array}$ & $\begin{array}{l}\text { vo2 } \\
\text { yo2 } \\
\text { Uo2 } \\
\text { Uo2 } \\
\text { UO2 } \\
\text { UO2 } \\
\text { UO2 } \\
\text { vo2 }\end{array}$ & $\begin{array}{l}\text { U235 } \\
\text { U235 } \\
\text { U235 } \\
\text { U235 } \\
\text { U235 } \\
\text { U235 } \\
\text { U235 } \\
\text { U235 }\end{array}$ \\
\hline HCAA14 & 1.01405 & 0.00391 & 24.5715 & H & 93.20 & $u$ & & $\mathbf{s}$ & CY & HOM & NITRATE & 1235 \\
\hline $\begin{array}{l}\text { HCASO2 } \\
\text { HCAS06 } \\
\text { HCAS07 } \\
\text { HCAS11 } \\
\text { HCAS18 } \\
\text { HCAS28 } \\
\text { HCAS32 } \\
\text { HCAS52 } \\
\text { HCAS60 } \\
\text { HCAS74 }\end{array}$ & $\begin{array}{l}1.01136 \\
1.00162 \\
1.01401 \\
0.99561 \\
1.00797 \\
1.01331 \\
0.97311 \\
0.98831 \\
1.00268 \\
1.02539\end{array}$ & $\begin{array}{l}0.00309 \\
0.00224 \\
0.00307 \\
0.00319 \\
0.00388 \\
0.00305 \\
0.00341 \\
0.00360 \\
0.00436 \\
0.00439\end{array}$ & $\begin{array}{r}8.5257 \\
24.9967 \\
11.7440 \\
9.4317 \\
21.5727 \\
7.7109 \\
24.2583 \\
24 . .2576 \\
22.5067 \\
22.1205\end{array}$ & $\begin{array}{l}H \\
H \\
H \\
H \\
H \\
H \\
H \\
H \\
H \\
H\end{array}$ & $\begin{array}{l}97.67 \\
93.20 \\
93.50 \\
93.10 \\
92.60 \\
93.20 \\
93.20 \\
93.20 \\
92.60 \\
92.60\end{array}$ & $\begin{array}{l}R \\
U \\
R \\
R \\
R \\
R \\
U \\
U \\
U \\
U \\
R\end{array}$ & $\begin{array}{l}\text { H2O } \\
\text { H2O } \\
\text { OIL } \\
\text { PLEX }\end{array}$ & $\begin{array}{l}\mathbf{S} \\
\mathbf{S} \\
\mathbf{S} \\
\mathbf{S} \\
\hat{A} \\
\hat{A} \\
\hat{A} \\
\hat{A} \\
\hat{A}\end{array}$ & $\begin{array}{l}\text { SP } \\
\text { SP } \\
\text { SP } \\
\text { SP } \\
C Y \\
C Y \\
\text { SL } \\
\text { SL } \\
C Y \\
C Y\end{array}$ & $\begin{array}{l}\text { HOM } \\
\text { HOM } \\
\text { HET } \\
\text { HET } \\
\text { HOM } \\
\text { HEI } \\
\text { HOW } \\
\text { HOM } \\
\text { HOM } \\
\text { HOM }\end{array}$ & $\begin{array}{l}\text { METAL } \\
\text { NITRATE } \\
\text { METAL } \\
\text { METAL } \\
\text { NITRATE } \\
\text { METAL } \\
\text { UOZF2 } \\
\text { UO2F2 } \\
\text { NITRATE } \\
\text { NITRATE }\end{array}$ & $\begin{array}{l}\text { U235 } \\
\text { U235 } \\
\text { U235 } \\
\text { U235 } \\
\text { U235 } \\
\text { U235 } \\
\text { U235 } \\
\text { U235 } \\
\text { U235 } \\
\text { U235 }\end{array}$ \\
\hline
\end{tabular}




\section{APPENDIX C. STATISTICAL ANALYSIS OF VALIDATION RESULTS}

0 input to statistical treatment

KENOV.a Validation 1-15-21. Low Enriched Experiments

proportion of the population $=.999$

confidence of $\mathrm{fit}=.950$

confidence on proportion $=.950$

number of observations $=48$

minimum value of $c$ losed bend $=\quad .00$

maximum value of closed band $=3.10199 \mathrm{E}-03$

0

estimated sample deviation $=3.10199 \mathrm{E}-03$
independent deviation

variable - $x$ variable $y$ in $y$

2.11690E+01

9.89660 .01

$3.96000 E-03$

$2.31190 \mathrm{E}+01$

9.893100 .01

$3.19000 \mathrm{E}-03$

2. $13940 \mathrm{E}+01$

$9.8055 \alpha$ or

$3.15000 \mathrm{E}-03$

$9.7923 \alpha$ or 2.53000 E -03

$2.31190 \mathrm{E}+01$

$2.53000 E-03$

9.893100 o

$3.19000 \mathrm{E}-03$

?.0000 of

$3.31000 \mathrm{E}-03$

$2.45660 \mathrm{E}+01$

9.0000 .

$3.24000 E-03$

9.87200 or

3. $40000 \mathrm{E}-03$

$2.28550 \mathrm{E}+01$

$3.10000 \mathrm{E}-03$

$9.915 \mathrm{rat} .01$

3.

.

$2.41000 \mathrm{E}-03$

$9.92600 \% \cdot 01$

$2.54000 E-03$

$2.47870 E+01$

$9.93210 \mathrm{C} \cdot 0 \mathrm{i}$

2.39000E-03

$1.55960 \mathrm{E}+01$

$9.00170 \mathrm{E}+00$

$2.95000 E-03$

2.70000 E- 03

$1.85660 \mathrm{E}+01$

$1.00500 E+00$

$2.94000 E-03$

$9.85370 E-01$

2.25000E-03

$9.86050 \mathrm{E}-01$

2.02000 - 03

$2.70000 \mathrm{E}-03$

9.94450E-01

.32790E+0

9.98780 E- 01

$2.78000 E-03$
$2.50000 E-03$

$2.32170 E+01$

$9.89750 \mathrm{E}-01$

$2.50000 E-03$
$2.72000 E-03$

$9.88530 \mathrm{E}-01$

$3.07000 \mathrm{E}-03$

$2.41060 \mathrm{E}+01$

$1.01240 E+00$

3.15000E-03

independent

variable - $x$

$2.17610 \mathrm{E}+01$

2. $35220 \mathrm{E}+01$

$2.32730 E+01$

.42200E+01

$2.42250 \mathrm{E}+01$

$2.42270 \mathrm{E}+0$

$2.13760 \mathrm{E}+0$

$2.11830 E+0$

$1.83380 E+01$

$1.92760 \mathrm{E}+01$

$2.15420 \mathrm{E}+01$

$2.01770 \mathrm{E}+01$

$1.85300 \mathrm{E}+01$

$1.93120 \mathrm{E}+01$

$2.18620 E+0$

$1.83290 \mathrm{E}+0$

$1.92220 E+0$

$1.91580 \mathrm{E}+0$

$1.97540 E+0$

$2.02680 E+0$

$1.98750 \mathrm{E}+01$

$1.97890 \mathrm{E}+0$

2. $15530 \mathrm{E}+01$

2. $14600 E+01$ dependent

variable - y

$1.00720 \mathrm{E}+00$

$1.00700 E+00$

$1.00430 \mathrm{E}+00$

9.93400E-01

9.91480E-01

$9.95460 E-01$

$1.01260 E+00$

$1.01150 E+00$

$1.01580 \mathrm{E}+00$

$1.00170 \mathrm{E}+00$

$1.00700 E+00$

$1.00810 \mathrm{E}+00$

9.99450E-01

$1.00900 E+00$

$1.00870 E+00$

$1.00360 \mathrm{E}+00$

$9.83000 E-01$

9.77150E-01

9.81780E-01

$9.96760 \mathrm{E}-01$

9.86440E-01

$9.87330 \mathrm{E}-01$

$1.00190 E+00$

9.92820E-01 deviation

in $y$

2.82000E-03

3.24000 - 03

$2.92000 \mathrm{E}-03$

$2.75000 E-03$

$3.05000 E-03$

$3.16000 \mathrm{E}-03$

3.08000 - 03

$3.20000 \mathrm{E}-03$

3.66000 E- 03

3.52000 E- 03

$3.25000 E-03$

2.96000 E -03

$3.02000 \mathrm{E}-03$

$3.42000 \mathrm{E}-03$

$3.12000 \mathrm{E}-03$

$2.63000 \mathrm{E}-03$

$3.52000 \mathrm{E}-03$

3.75000 E -03

3.10000E-03

$4.29000 \mathrm{E}-03$

3.06000E-03

3.40000 - 03

$3.04000 E-03$

$3.70000 \mathrm{E}-03$

0 KENOV Verlide

Student $t=9.680 E+00$ for 46 dof's and $P=.950$.

subrout ine fit

xbar $=2.1598 E+01$, standard deviation in xbar $=2.3617 \varepsilon+00$

ybar $=9.9628 E-01$, standard deviation in yar $=9.9289 \mathrm{E}-03$

sigmaxx $=2.6215 \mathrm{E}+02$, si gmayy $=0.6334 \mathrm{E}-03$, sigmaxy $=-1.6518 \mathrm{E}-01$

root mean square error of $i t=$

$r$ squared of $f$ it $=2.2464 \mathrm{E}-02$

standard variance of the $f$ it

standard variance of the $f$ it

$=$

est imated sample variance

$9.84642 \mathrm{E}-05$

estimated total standard error $=1.03965 \mathrm{E}-02$

Student $t=1.679 E+00$ for 47 dof's and $P=.950$

student $t=3.090 E+00$ for 150 dof's and $p=.999$

estimate of lower 95.000 percent confidence

bound on 99.900 percent proportion of the

population for the data treated as a single group

osc $=9.5685 \mathrm{E}-01$

subroutine fnorm

bin tallies $11.0 \quad 12.0 \quad 3.0 \quad 10.0 \quad 12.0$

WARNING th* the test for normal may be unreliable

chi $=5.9583$ compared to 9.49 . The data tests normal

Student $t=3.690 E+00$ for 150 dof's and $P=.999$.

Chi*t2 $=2.920 E+01$ for 46 dof's and $P=.025$ :

subrout ine conf

$g=.3978, h=.2442$, rho $=-.5372$

surout ine $d$ calc

gamma $=.050 \quad A=6139, n=48$ rho $=.53718$

$d=1.00^{\circ}$, ans $=.3439^{\circ}$, nof $=1689$

$d=1.00$, ans $=1.3439$, nof $=1089$
$d=10.00$, ans $=1.0000$, nof $=2145$

\begin{tabular}{cccccc}
$d 1$ & $d$ & $d 2$ & a1 & ans & a2 \\
\hline 1.0000 & 10.0000 & 10.0000 & .3439 & .9500 & 1.0000 \\
1.0000 & 5.5000 & 5.5000 & .3439 & .9500 & .9985 \\
3.2500 & 3.2500 & 5.5000 & .9469 & .9500 & .9985 \\
3.2500 & 4.3750 & 4.3750 & .9469 & .9500 & .9899
\end{tabular}




$\begin{array}{llllll}3.2500 & 3.8125 & 3.8125 & .9469 & .9500 & .9761 \\ 3.2500 & 3.5313 & 3.5313 & .9469 & .9500 & .9641 \\ 3.2500 & 3.3906 & 3.3906 & .9469 & .9500 & .9562 \\ 3.2500 & 3.3203 & 3.3203 & .9469 & .9500 & .9518 \\ 3.2852 & 3.2852 & 3.3203 & .9494 & .9500 & .9518 \\ 3.2852 & 3.3027 & 3.3027 & .9494 & .9500 & .9506 \\ \text { Jumped out, } n=0 & 10 & & & \end{array}$

subroutine conf Selected $D$ value =

\section{$3.294 E+00$}

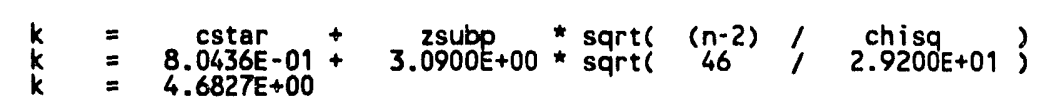

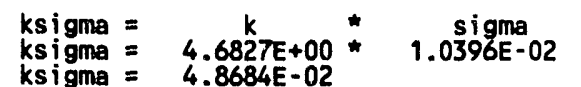

1 output from statistical trestment

KENOV.a Validation 1-15-91. Low Enriched Experiments

linear lcast squares fit to data

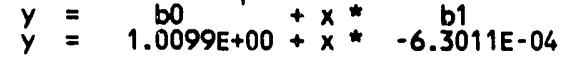

[T for $H 0: b 1=0.\}=-1.0281 E+00$

095.0 percent confidence of the fit (not plotted)

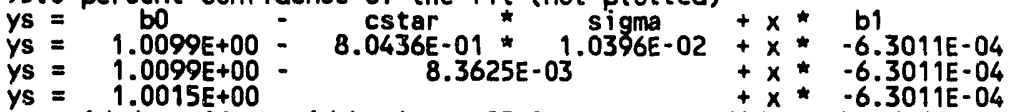

0 one-sided, uniform-width, lower 95.0 percent confidence band for a single future calculation

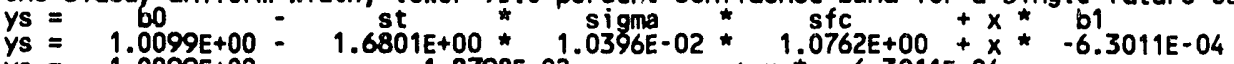
$y s=1.0099 E+00-1.601 E+001.8798 E-02-0396 E-02 *-6.3011 E-04$

0 lower toilerance bound for

95.0 percent confidence on 99.9 percent of the population

$0 \mathrm{yc}=$ bo

$y c=1.0099 E+00-4.8684 \mathrm{E}-02+x *-6.3011 \mathrm{E}-04$

$y c=9.6120 \mathrm{E}-01+x+6.3011 \mathrm{E}-04$

0 margin between the confidence band for a single

future calculation and the lower tolerance bound 2.9885E-02

095.0 percent confidence on a single future calculation (not plotted)

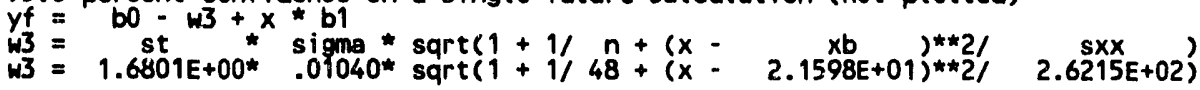

minimm value of $c$ losed band $=1.5596 \mathrm{E}+01$

maximm value of closed band $=2.4787 \mathrm{E}+01$

minimum value of $y=9.7715 \mathrm{E}-01$

maximum value of $y=1.0158 E+00$

o input to statistical treatment

O KENOV.a Validation 1-15-91. High Enriched Experiments

proportion of the population $=.999$

confidence of $\mathrm{fit}=.950$

confidence on proportion $=.950$

number of observations $=11$

minimum value of closed band $=\quad .00$

maximum value of closed band $=.00$

0 est imated sample deviation $=3.52500 \mathrm{E}-03$

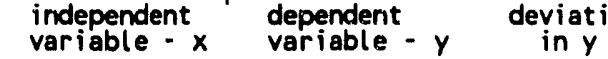

\section{$2.45720 E+01 \quad 1.01410 E+00 \quad 3.91000 E-03$}

$\begin{array}{lll}8.52570 \mathrm{E}+00 & 1.01140 \mathrm{E}+00 & 3.09000 \mathrm{E}-03 \\ 2.49970 \mathrm{E}+01 & 1.00160 \mathrm{E}-\mathrm{j} 0 & 2.24000 \mathrm{E}-03\end{array}$

$1.17440 \mathrm{E}+01 \quad 1.01400 \mathrm{E}+00 \quad 3.07000 \mathrm{E}-03$

$9.43170 \mathrm{E}+00 \quad 9.95610 \mathrm{E}-01 \quad 3.19000 \mathrm{E}-03$

$2.15730 \mathrm{E}+01 \quad 1.00800 \mathrm{E}+00 \quad 3.88000 \mathrm{E}-03$

$7.71090 \mathrm{E}+00 \quad 1.01330 \mathrm{E}+00 \quad 3.05000 \mathrm{E}-03$

$2.42580 \mathrm{E}+01 \quad 9.73110 \mathrm{E}-01 \quad 3.41000 \mathrm{E}-03$

$2.42580 \mathrm{E}+01 \quad 9.88310 \mathrm{E}-01 \quad 3.4000 \mathrm{E}-03$

$2.42580 \mathrm{E}+01 \quad 9.88510 \mathrm{E}-01 \quad 3.60000 \mathrm{E}-03$

$\begin{array}{lll}2.25070 E+01 & 1.00270 E+00 & 4.36000 E-03 \\ 2.21210 E+01 & 1.02540 E+00 & 4.39000 E-03\end{array}$

1 intermediate calculational results

o rENOV.a Validation 1-15-91. High Enriched Experiments

student $t=1.833 E+00$ for 9 dof's and $P=.950$.

subroutine $f$ it

xbar $=1.8336 \mathrm{E}+01$, standard deviation in xbar $=7.2627 \mathrm{E}+00$

ybar $=1.0043 \mathrm{E}+00$, standard deviation in ybar $=1.4472 \mathrm{E}-02$ 


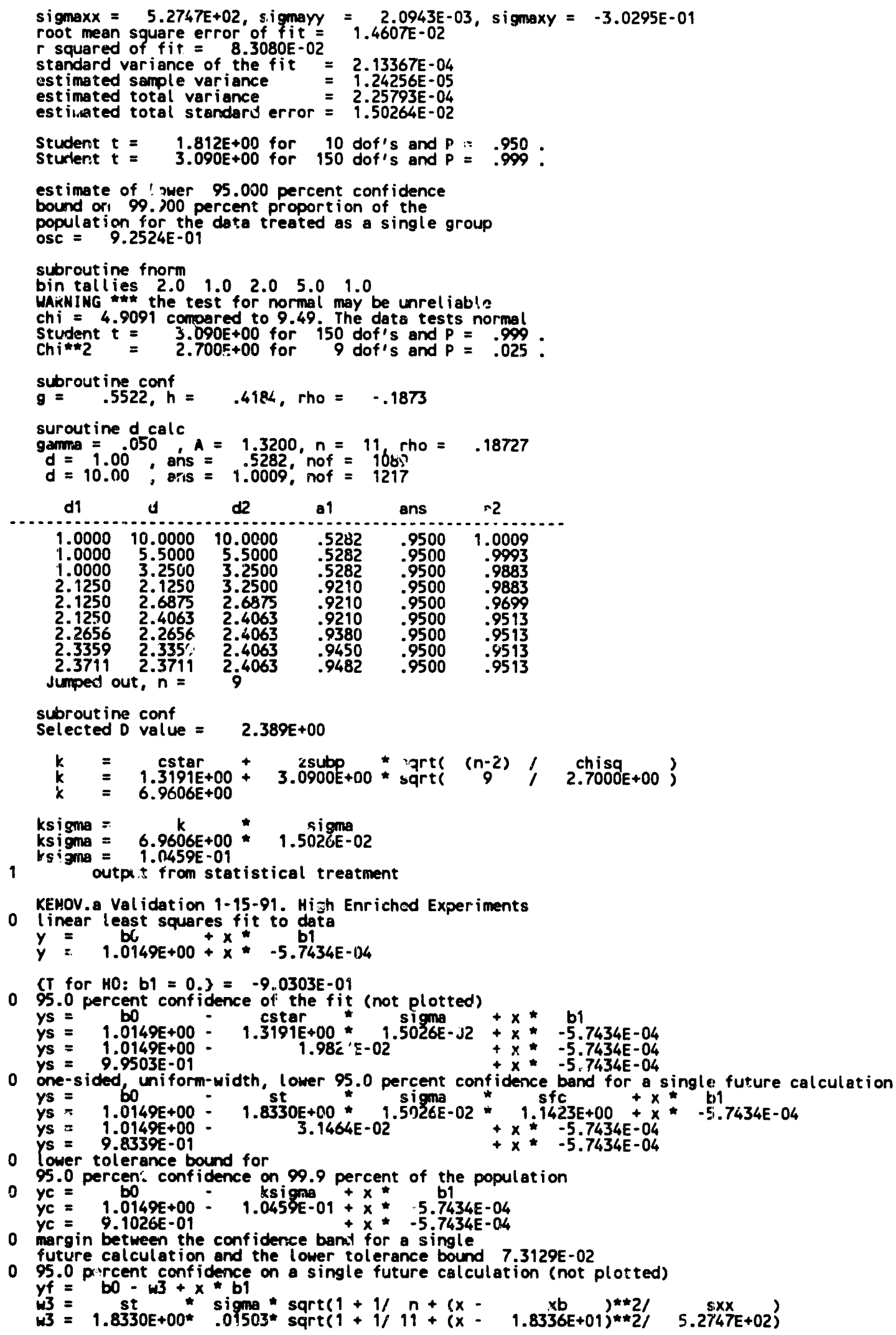

0 linear least squares fit to data $y=b C$
$y=1.0149 E+00+x *-5.7434 E-04$

(T for HO: $b 1=0.3=-9.0303 E-01$

095.0 percent confidence of the fit (not plotted)

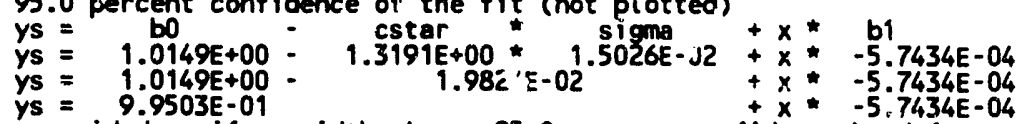

0 one-sided uniform-width, lower 95.0 percent confidence band for a single future calculation

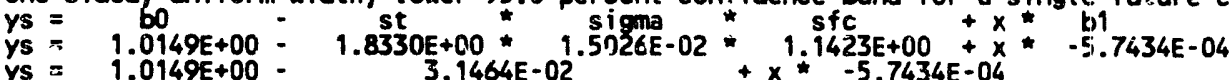
ys $=1.0149 E+00$

lower tolerance bound for 95.0 percen: confidence on 99.9 percent of the population

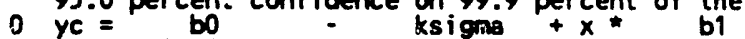

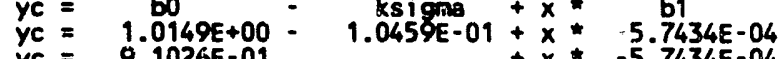
$y c=9.1026 E-01+x+-5.7434 E-0.4$

0 margin between the confidence bant for a single future calculation and the lower tolerance bound 7.3129E-02

095.0 parcent confidence on a single future calculation (not plotted)

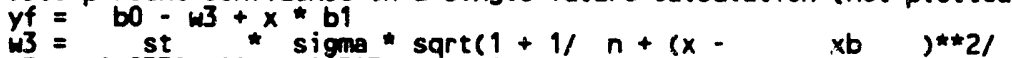

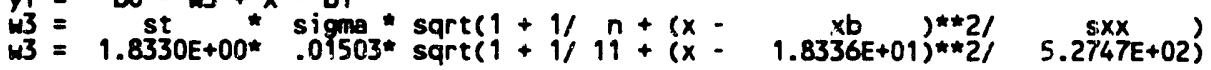


minimum value of $c$ losed band $=$ $\begin{array}{ll}\operatorname{minimm} & \text { value of } y \\ \text { maximum value of } y & =\end{array}$ .4997E+0 $9.7311 E-01$
$1.0254 E+00$ input to statistical treatment

KENOV. Validation 1-15-91. High and Low Enriched Experiments

$\begin{aligned} \text { proportion of the population } & =.999 \\ \text { confidence of } \mathrm{fit} & =.950\end{aligned}$

confidence on proportion $=.950$

number of observations $=59$

minimum value of closed band $=\quad .00$

estimated sample deviation $=3.18512 \mathrm{E}-03$

0

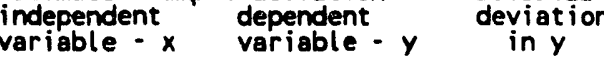

2.11690E+01

9.89440E-01

$3.96000 E-03$
$3.19000 E-03$

. $31190 \mathrm{E}+01$

$9.89310 \mathrm{E}-0$

3.15000E-03

$2.53000 E-03$

. 005 L

2.4055OE+01

$9.79250 E-01$

$3.56000 E-03$

$2.33600 E+01$

$9.91270 \mathrm{E}-01$

$3.56000 E-03$
$3.31000 E-03$

$3.24000 \mathrm{E}-03$

$3.40000 \mathrm{E}-03$

$9.99880 \mathrm{E}-0$

3. 10000 E- 03

$2.41000 \mathrm{E}-03$

$9.85950 \mathrm{E}-0$

$9.91570 E-01$

2. 30000 -

$2.95000 \mathrm{E}-03$

$9.92460 \mathrm{E}-0$

$2.70000 E-03$

$2.94000 E-03$

2.25000E-03

$2.02000 E-03$

2.70000E-03

2.78000E-03

2.50000E-03

2.72000E-03

3.07000E-03

$3.15000 E-03$

2.82000E- 03

$3.24000 E-03$

2.92000E - 03

2. $75000 \mathrm{E}-03$

$3.05000 E-03$

$3.16000 E-03$

1'

$9.91480 \mathrm{E}-01$

9.95460E-0

KEN.8 Validation 1-15-91. High and Low Enriched Experiments

subrout ine fit

xbar $=2.0990 E+01$ standard deviation in xbar $=3.9059 E+00$

xbar $=2.0990 E+01$, standard deviation in xbar $=3.9059 E+00$
ybar $=9.9778 E-01$ standard deviation in ybar $=1.1224 \mathrm{E}-02$

ybar $=9.9778 E-01$, standard deviation in ybar $=1.1224 \mathrm{E}-02$
sigmaxx $=8.8483 \mathrm{E}+02$, sigmayy $=7.3069 \mathrm{E}-03$, sigmaxy $=-7.0295 \mathrm{E}-01$

root mean square error' of fit $=1.0881 \mathrm{E}-02$

$r$ squared of $\mathrm{fit}=7.6429 \mathrm{E}-02$

standard variance of the fit $=1.18394 \mathrm{E}-04$

estimated sempie variance $=1.01450 \mathrm{E}-05$

estimated total variance $=1.285395-04$

est imated total standard error $=1.13375 \mathrm{E}-02$

Student $t=1.672 E+00$ for 58 dof's and $P=.950$.
Student $t=3.090 E+00$ for 150 dof's and $P=.999$.

estimate of lower 95.000 percent confidence

bound on 99.900 percent proportion of the

population for the dita treated as a single group

OSC $=0.5455 \mathrm{E}-01$

subrout ine fnorm

bin tallies $13.013 .0 \quad 8.012 .013 .0$

chi $=1.5932$ compared to 9.49 . The data tests normal

Student $t=3.090 \mathrm{E}+00$ for 150 dof's and $P=.999$

Chi*2 $=3.814 E+01$ for 57 dof's and $P=.025$

subrout ine conf

$g=.4650, h=.1873$, rho $=-.4958$

suroutine d calc

gema $=.050, A=.4029, n=59$, rho $=$

.49577

independent
variable - $x \quad \begin{gathered}\text { dependent } \\ \text { variable }-y\end{gathered}$ in $y$

$2.13760 E+019.01260 E+00 \quad 3.08000 E-03$

$1.01150 E+00$

$1.01580 E+00$

$1.00170 E+00$

$1.00700 \mathrm{E}+00$

$1.00810 \mathrm{E}+00$

$9.99450 \mathrm{E}-01$

$1.00900 \mathrm{E}+00$

$1.00870 \mathrm{E}+00$

$1.00360 \mathrm{E}+00$

9.83000E-01

9.77150E-0

$9.81780 E-01$

$9.96760 \mathrm{E}-01$

.86440E-0

$9.87330 \mathrm{E}-0$

$1.00190 \mathrm{E}+00$

$9.92820 \mathrm{E}-01$

$1.01410 E+00$

$1.01140 E+00$

$1.00160 \mathrm{E}+00$

$1.01400 E+00$

9.95610 - 01

$1.00800 E+00$

$1.01330 E+00$

9.73110E-O

$9.88310 E-01$

$1.00270=+00$

$1.02540 \mathrm{E}+00$

$6000-03$

$3.52000 E-03$

3.25000E-03

3. $25000 E-03$

. $96000 E-03$

3.02000E-03

$3.42000 E-03$

$3.12000 \mathrm{E}-03$

$2.63000 E-03$

3.52000E- 03

$3.75000 E-03$

3.10000E-03

$4.29000 E-03$

$3.06000 \mathrm{E}-03$

3. $40000 \mathrm{E}-03$

3. $04000 \mathrm{E}-03$

$3.70000 E-03$

3.91000 E- 03

3.09000 E- 03

2.24000 - 03

$3.07000 \mathrm{E}-03$

3.19000E-03

3.88000 E- 03

$3.05000 \mathrm{E}-03$

$3.41000 \mathrm{E}-03$

$3.60000 \mathrm{E}-03$

$4.36000 \mathrm{E}-03$

$4.39000 \mathrm{E}-03$ 


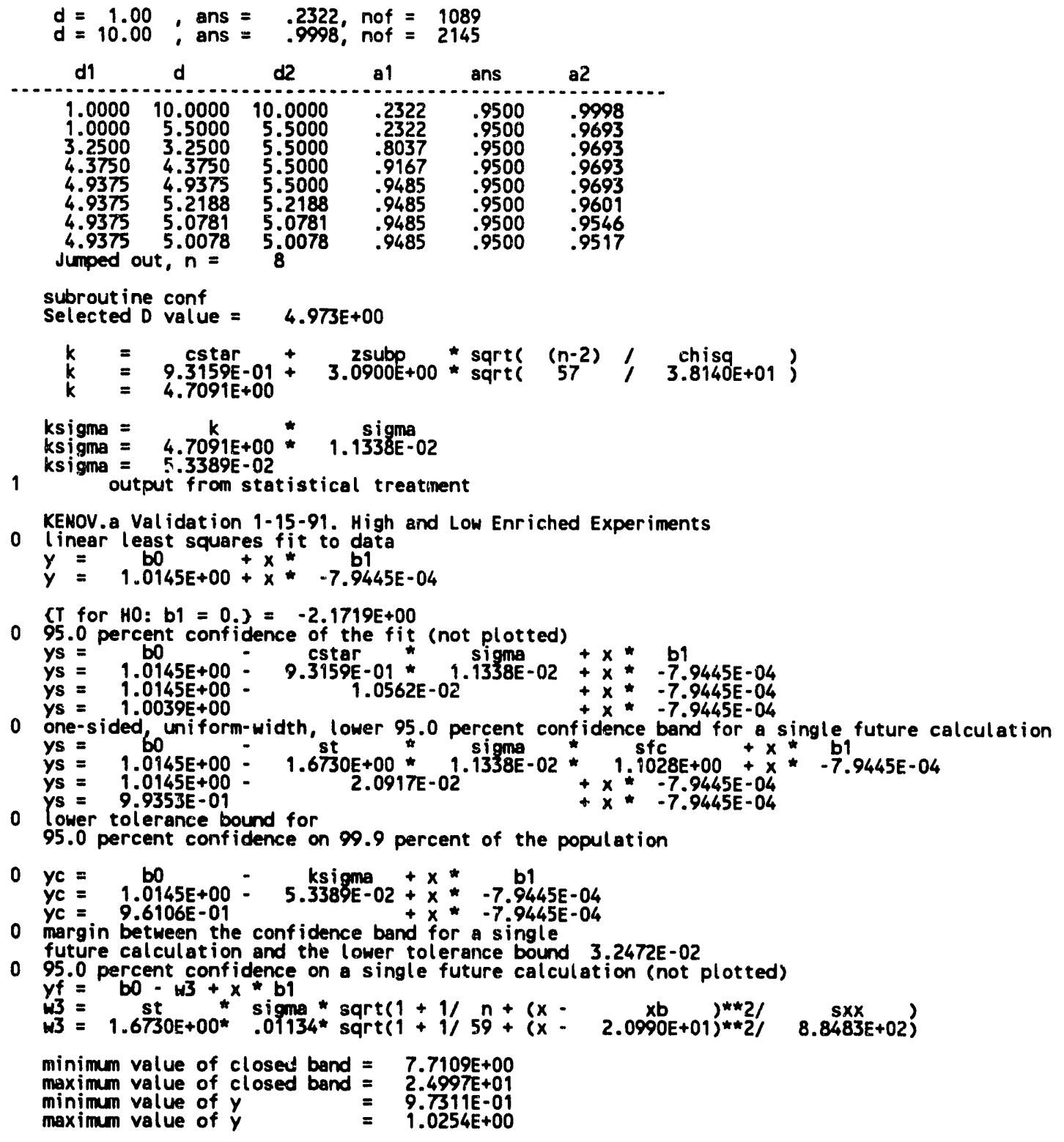




\section{INTERNAL DISTRIBUTION}

1. T. A. Angelelli

2. D. Baltimore

3. E. J. Barber

4. S. M. Bowman

5. W. R. Brock

6. L. D. Charles

7. W. K. Crowley

8-10. D. M. D'Aquilla

11. J. C. Dean

12-13. H. R. Dyer

14. D. A. Dyslin

15. D. L. English

16. P. A. Haas

17. H. F. Hartman

18-20. T. W. Hines

21. J. A. Hoffmeister

22. J. C. Ingram III

23-32. W. C. Jordan

33. R. A. Just

34. M. R. Lambert

35. P. D. Lassiter
36. J. P. Little, Jr.

37. S. K. Martin

38. D. S. Milewski

39. R. L. Newvahner

40. L. F. Norris

41. M. G. Otey

42-43. C. V. Parks

44. A. J. Saraceno

45-46. C. H. Shappert

47. J. N. Sumner

48. C. A. Sweet

49. R. G. Taylor

50. D. A. Walker

51. B. G. Warriner

52. R. M. Westfall

53. G. E. Whitesides

54. D. W. Wilson

55-56. Laboratory Records Dept.

57. Laboratory Records, ORNL-RC

58. ORNL Y-12 Research Library Document Reference Section

59. Central Research Library

60. ORNL Patent Section

\section{EXTERNAL DISTRIBUTION}

61. Office of the Assistant Manager for Entigy Research and Development, U.S. Department of Energy Field Office, Oak Ridge, (DOE-OR), P.O. Box 2001, Oak Ridge, TN 37831

62-63. Office of Scientific and Technical Information, U.S. Department of Energy, P.O. Box 62, Oak Ridge, TN 37831 

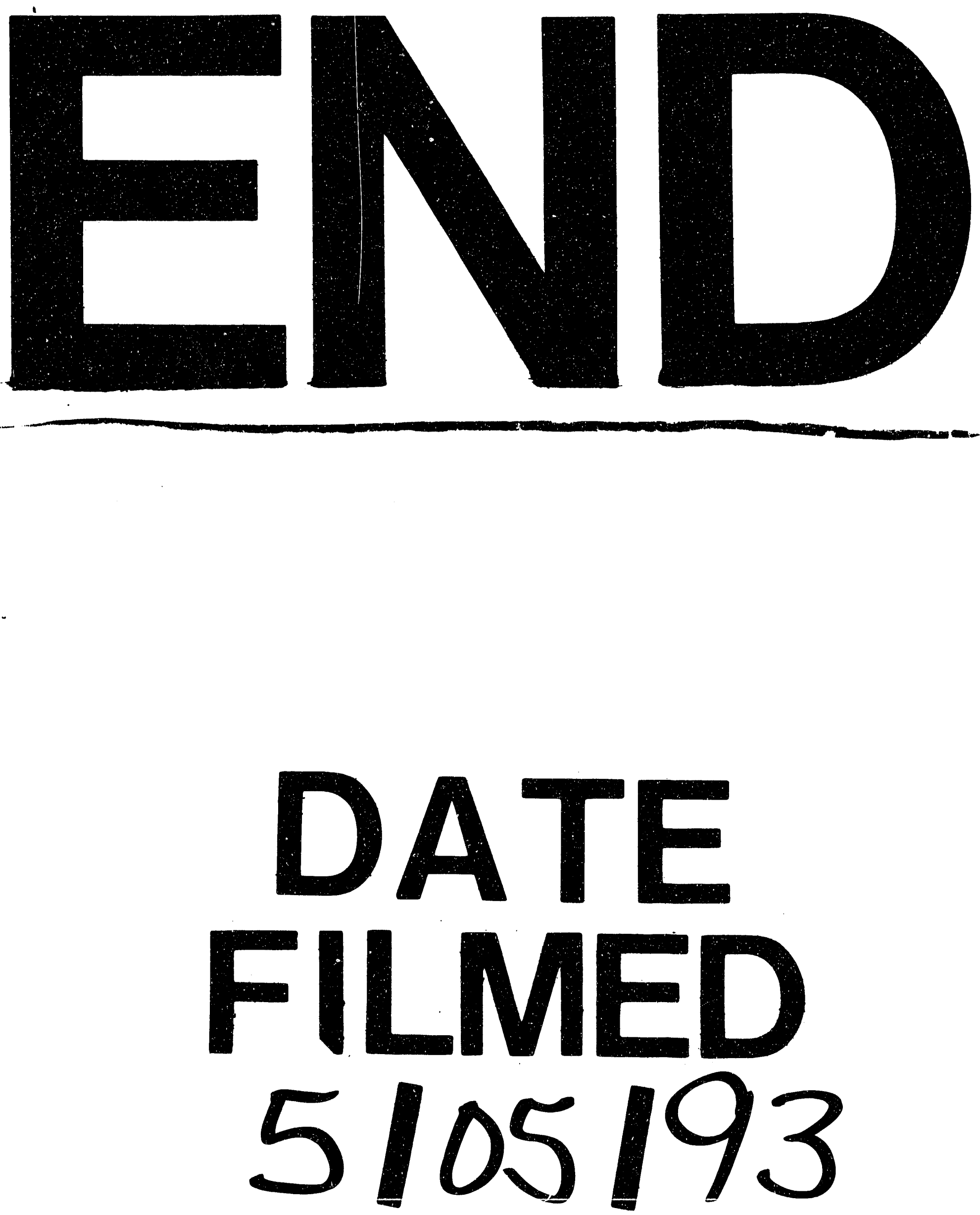
| 